\title{
High-sensitivity cardiac troponins in heart and kidney diseases: from lab to clinic
}

Citation for published version (APA):

Cardinaels, E. P. M. (2015). High-sensitivity cardiac troponins in heart and kidney diseases: from lab to clinic. [Doctoral Thesis, Maastricht University]. Uitgeverij BOXPress.

https://doi.org/10.26481/dis.20150710ec

Document status and date:

Published: 01/01/2015

DOI:

10.26481/dis.20150710ec

Document Version:

Publisher's PDF, also known as Version of record

\section{Please check the document version of this publication:}

- A submitted manuscript is the version of the article upon submission and before peer-review. There can be important differences between the submitted version and the official published version of record.

People interested in the research are advised to contact the author for the final version of the publication, or visit the DOI to the publisher's website.

- The final author version and the galley proof are versions of the publication after peer review.

- The final published version features the final layout of the paper including the volume, issue and page numbers.

Link to publication

\footnotetext{
General rights rights.

- You may freely distribute the URL identifying the publication in the public portal. please follow below link for the End User Agreement:

www.umlib.nl/taverne-license

Take down policy

If you believe that this document breaches copyright please contact us at:

repository@maastrichtuniversity.nl

providing details and we will investigate your claim.
}

Copyright and moral rights for the publications made accessible in the public portal are retained by the authors and/or other copyright owners and it is a condition of accessing publications that users recognise and abide by the legal requirements associated with these

- Users may download and print one copy of any publication from the public portal for the purpose of private study or research.

- You may not further distribute the material or use it for any profit-making activity or commercial gain

If the publication is distributed under the terms of Article $25 \mathrm{fa}$ of the Dutch Copyright Act, indicated by the "Taverne" license above, 
High-sensitivity cardiac troponins in heart and kidney diseases:

from lab to clinic 
Lay-out, cover illustration and design by Eline Cardinaels Printed by Proefschriftmaken.nl || Uitgeverij BOXPress Published by: Uitgeverij BOXPress, `s Hertogenbosch

(C) Eline Cardinaels, Maastricht, 2015 ISBN: 978-94-6295-206-5 


\title{
High-sensitivity cardiac troponins in heart and kidney diseases: from lab to clinic
}

\author{
PROEFSCHRIFT \\ Ter verkrijging van de graad van doctor aan de Universiteit Maastricht, \\ op gezag van de Rector Magnificus, Prof. dr. L.L.G. Soete \\ volgens het besluit van het College van Decanen, \\ in het openbaar te verdedigen op
}

Vrijdag 10 juli 2015 om 14.00 uur

Door

Eline Pieter Maria Cardinaels

Geboren op 18 juni 1986 te Bree, België 


\section{Promotor:}

Prof. dr. M.P. van Dieijen-Visser

\section{Co-promotoren:}

Prof. dr. O. Bekers

Dr. ir. A.M.A. Mingels

\section{Beoordelingscommissie:}

Prof. dr. L.J. van Loon (voorzitter)

Dr. S.C. Bekkers

Prof. dr. J.F. Glatz

Prof. dr. G.C.M. Kusters (Jeroen Bosch Ziekenhuis, 's-Hertogenbosch)

Prof. dr. C. Mueller (University Hospital Basel, Switzerland)

The kind contribution of Roche Diagnostics, Abbott Diagnostics and Beckman Coulter to thesis is greatly acknowledged. 


\section{Table of contents}

CHAPTER ONE

CHAPTER TWO

CHAPTER THREE

CHAPTER FOUR

CHAPTER FIVE

CHAPTER SIX

CHAPTER SEVEN

CHAPTER NINE

ADDENDUM-1

ADDENDUM -2
General Introduction

7

A comprehensive review of upper reference

25 limits reported for (high-) sensitivity cardiac troponin assays: the challenges that lie ahead

High-sensitivity cardiac troponin concentrations in patients with chest discomfort: is it the heart or the kidneys as well?

High-sensitivity cardiac troponin T differs from 79 troponin I in the association with renal dysfunction and mortality in nursing home residents

Acute effects of conventional and extended hemodialysis and hemodiafiltration on highsensitivity cardiac troponins

Time-dependent degradation pattern of cardiac troponin $\mathrm{T}$ following myocardial infarction

115

Accumulation of $\mathrm{N}$ - terminal truncated cardiac 137 troponin $\mathrm{T}$ fragments in the serum of patients with end-stage renal disease

General Discussion

153

Summary

Samenvatting

178

Valorisation

183

Abbreviations

193

Dankwoord - Acknowledgments

Curriculum Vitae 



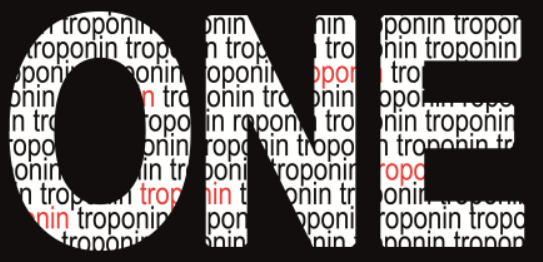

\section{GENERAL INTRODUCTION}



Acute myocardial infarction (AMI), also known as a heart attack, is a prevalent cardiovascular disease and a major cause of hospitalisations and deaths [1-3]. AMI reflects the loss of cardiac tissue as a result of prolonged ischemia that originates from an imbalance between blood supply and demand [4]. Ultimately this will result in the manifestation of symptoms such as chest pain and dyspnea. Blood supply is typically decreased because of a ruptured atherosclerotic plaque in the coronary arteries (coronary artery disease; CAD), subsequently forming a thrombus that can occlude the coronary blood vessel (FIGURE 1). However, also conditions other than CAD can provoke myocardial damage and AMI, such as coronary vasospasms, endothelial dysfunction and revascularization procedures.

A. healthy heart

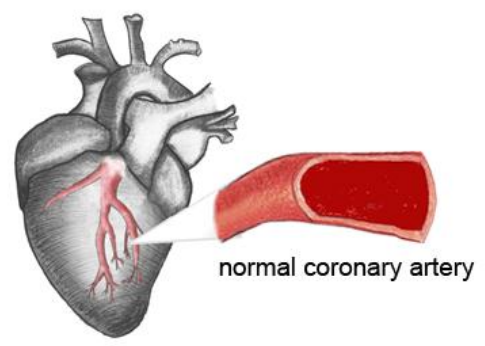

B. acute myocardial infarction

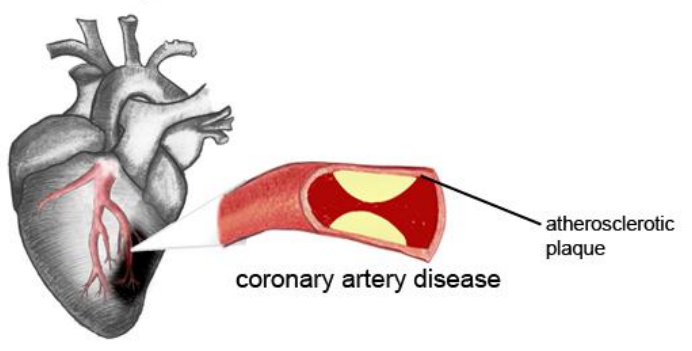

FIGURE 1. Representation of normal coronary arteries in a healthy heart (A) and the presence of coronary artery disease (CAD) leading to acute myocardial infarction (AMI) (B).

Cardiac biomarkers are an essential component of the diagnostic criteria for myocardial infarction [4]. Ideally, to make a correct and early diagnosis, a cardiac biomarker should be heart specific and early detectable in the patients' blood [5]. Many previous biomarkers (such as creatine kinase and lactate dehydrogenase) have the disadvantage that they are not solely expressed in the heart. Therefore, in the early 1990s, the search for a better cardiac biomarker has led to the discovery of cardiac specific isoforms of troponin T $(c \operatorname{TnT})[6,7]$ and troponin I (cTnl) [8]. 


\section{(Patho-)physiology of cardiac troponins}

Troponins are components of the sarcomeres, where they regulate the contraction and relaxation of muscle tissue [9] (FIGURE 2). They are composed of three subunits: troponin $\mathrm{T}$, which anchors the protein-complex to tropomyosin; troponin I, facilitating the binding of myosin heads to actin strains; and troponin $\mathrm{C}$, the calcium-binding subunit that initiates muscle contraction. Troponins $T$ and I both have a unique isoform that is exclusively expressed in cardiac muscle (cTnT and cTnl, respectively), whereas troponin $C(T n C)$ has not. Structural characterisation of $\mathrm{cTnT}$ and $\mathrm{cTnl}$ revealed two elongated proteins with a relatively low molecular weight of approximately $38 \mathrm{kDa}$ and 24 $\mathrm{kDa}$, respectively $[9,10]$.

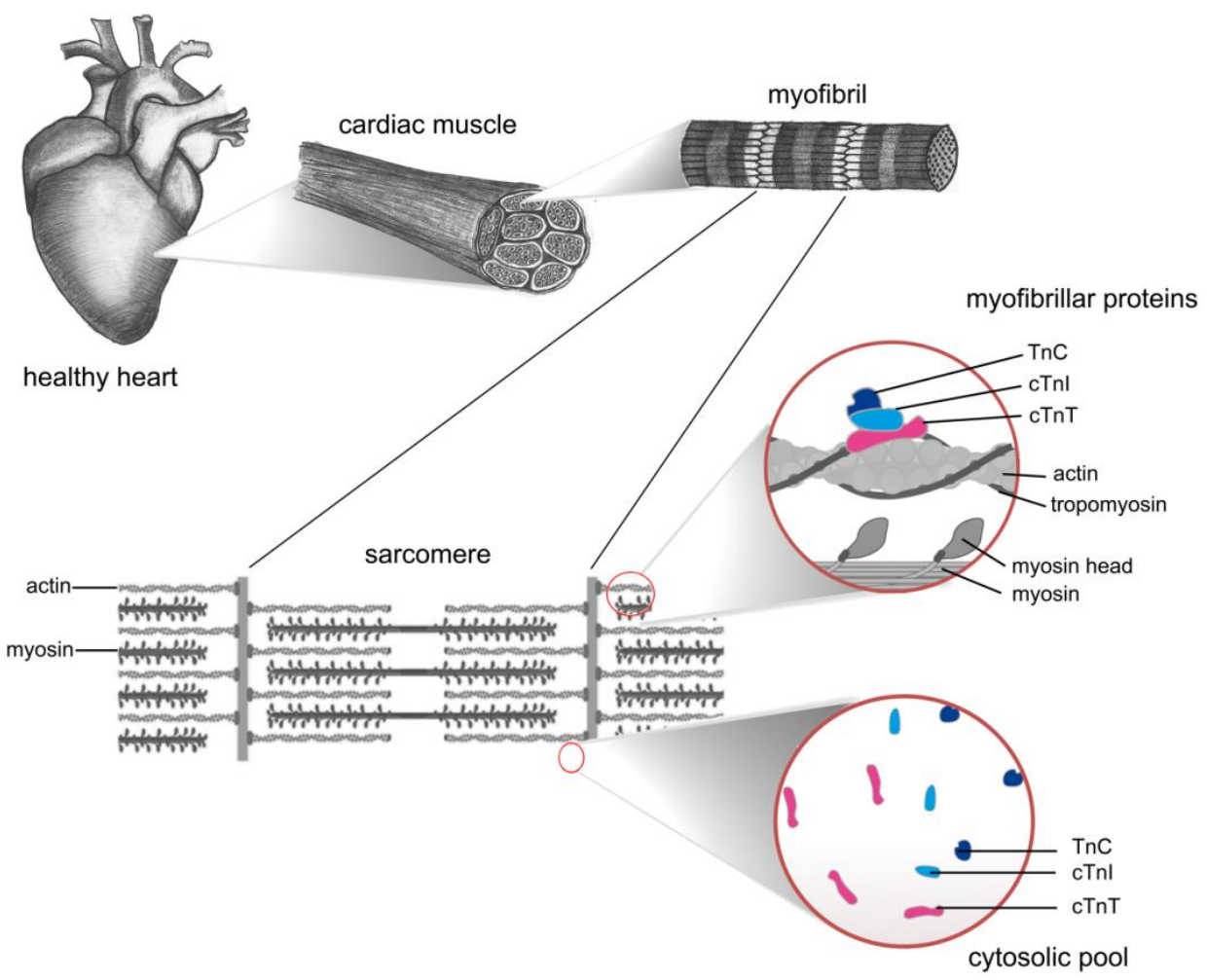

FIGURE 2. Physiology of the healthy myocardium. The myocardium is composed of bundles of myofibrils, which in turn are comprised of repeating units of sarcomeres. Subsequently, each sarcomere contains myosin and actin filaments which are bound at regular sites to the CTnT-I-C complexes. 
Within the cardiomyocytes, cardiac troponins (cTn) are complexed with the cardiac myofibrils, although there is some evidence for a smaller soluble fraction (5-10\% of total cTn amount) in the cytoplasm [11-13]. Recently, it was shown that this cytosolic fraction is probably not a fixed percentage, but may depend on a seemingly reversible binding between cTn and myofibrilar proteins [14]. Also, in vitro studies have demonstrated that the presence of unbound CTnT induces cytotoxicity to the cardiomyocytes [15], questioning whether such soluble fractions truly exists.

Ischemic damage to cardiomyocytes causes increased cell permeability and necrosis, resulting in a rapid release of cTn molecules into the patients' blood circulation (FIGURE 3).

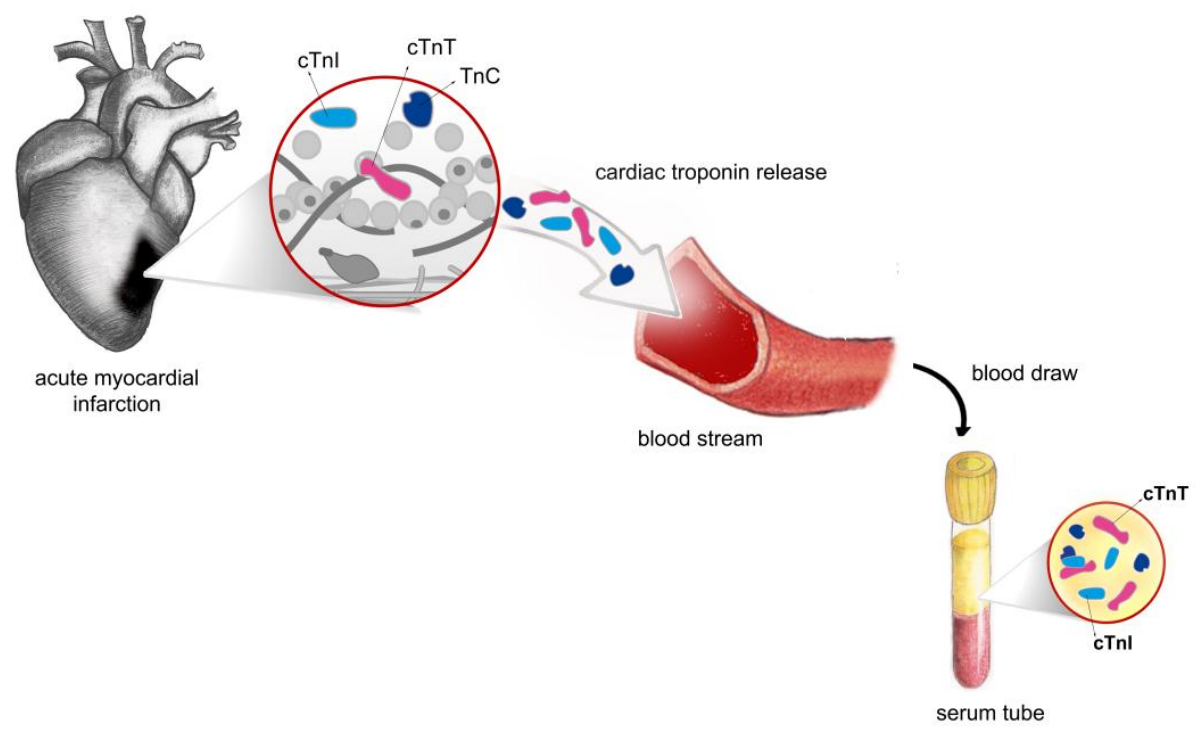

FIGURE 3. Following myocardial damage, cardiac troponins are released into the patients' blood stream. The cTn proteins can be measured in blood samples collected from the patient. 
Once released into the blood, cTnT and cTnl can be measured using antibodydependent assays (FIGURE 4). Serial cTnT measurements in AMI patients' sera reveal a distinct biphasic release pattern. This cTnT-curve initially displays a rapid increase in just a few hours after $\mathrm{AMI}$, and a second prolonged elevation or plateau phase in which cTn concentrations remain abnormal for up to two weeks $[11,16,17]$. It is hypothesized that the initial release originates from the cytosolic cTnT pool, which are easily released into the blood circulation $[11,13]$. The sustained cTnT concentrations are presumed to be the result of slower release of myofibril-bound cTn molecules. In contrary to cTnT, cTnl concentrations more rapidly decreases after reaching peak concentrations, resulting in a monophasic release curve [17].

\section{cTnT-assay}

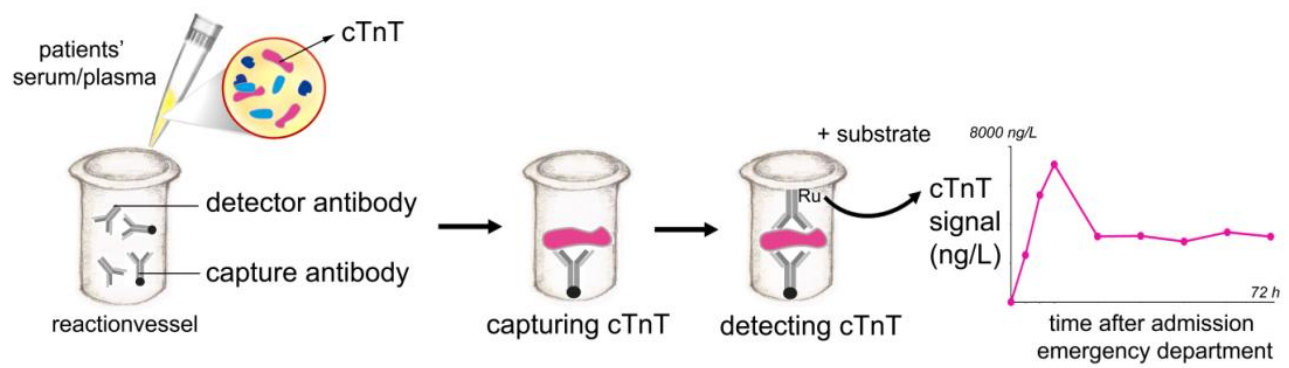

\section{cTnl-assay}

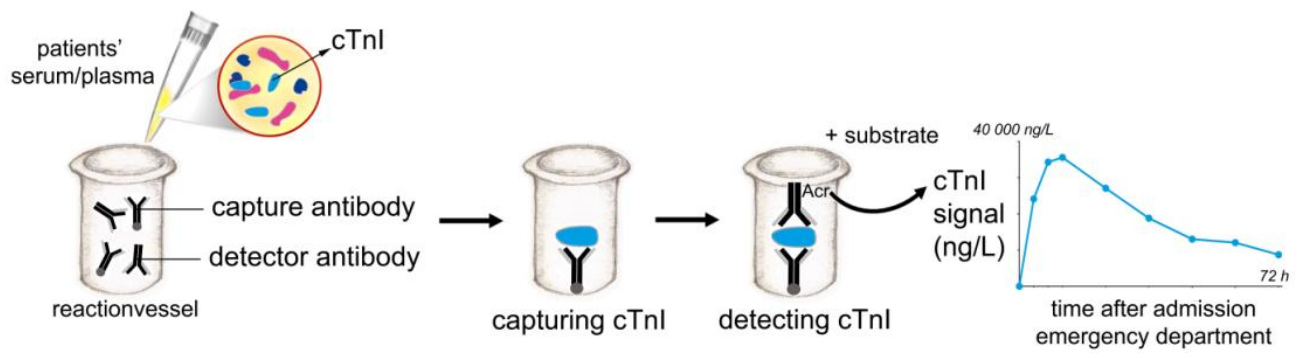

FIGURE 4. Measuring principles of cTnT and most cTnl assays. Generally, circulating cTn proteins in the patients' blood are captured and detected using antibodies specifically directed against cTn. Serial cTnT or cTnl measurements in AMI patients' blood result in a biphasic or monophasic release curve, respectively (PROTECT study, Maastricht University Medical Center, unpublished data). 
Ischemia is also known to activate proteolytic enzymes that can result into posttranslational modifications of cTnT and cTnl proteins, such as phosphorylation and degradation [18]. A heterogeneous mixture of molecular forms of cTnT and cTnl proteins have been characterized in the blood of AMI patients [5]. For cTnl, free and complex forms with TnC (cTnl-C complex) and to a lesser extent with cTnT (cTnT-I-C complex) were observed [19-21]. Also fragments of cTnl as well as cTnT have been described [16, 22, 23]. Although the presence of cTnT fragments were strongly disputed by Bates and colleagues, who found cTnT to mainly circulate in the intact free form [24]. Whether cTnT fragments are present in the patient's circulation therefore remained a matter of debate.

\section{Cardiac troponin as the diagnostic biomarker for acute myocardial infarction}

According to consensus documents of multiple international societies in cardiology [4, 25, 26] and biochemistry [27, 28], cTnT and cTnl are since 2000 the preferred biochemical markers to diagnose AMI because of their tissue specific expression in the myocardium. In addition, they should be accompanied with clinical symptoms or imaging signs of ischemia, that can be assessed by a number of different means such as physical examinations, changes on electrocardiography (ECG) and imaging evidence of new loss of viable myocardium [4]. AMI patients can be subdivided according to the presence or absence of ST-elevation on the ECG, resulting in ST-elevation MI (STEMI) and non-ST-elevation MI (non-STEMI). Because of the absence of ECG changes, NSTEMI is more difficult to diagnose and mainly relies on the presence of elevated cTn concentrations [6, 29].

The diagnostic cTn cutoff for myocardial infarction was set at the $99^{\text {th }}$ percentile upper reference limit (URL) of a healthy reference population (FIGURE 5) [25]. It was presumed that the amount of false positives is minimized in this way, as it is more common for other biomarkers to use the $97.5^{\text {th }}$ percentile URL. The diagnostic cutoff should also be measured with sufficient precision, preferably not exceeding a $10 \%$ coefficient of variation (CV) for the cTn assays although up to $20 \%$ CV remains clinically acceptable [28, 30].

The cTnT assay is produced by a single manufacturer, while multiple companies $(>10)$ market cTnl assays. Each manufacturer uses different 
antibody pairs [31], resulting in variable assay results [32]. Due to the heterogeneity in cTnl assays, it is strongly endorsed for each assay to report their own $99^{\text {th }}$ percentile concentration [33].

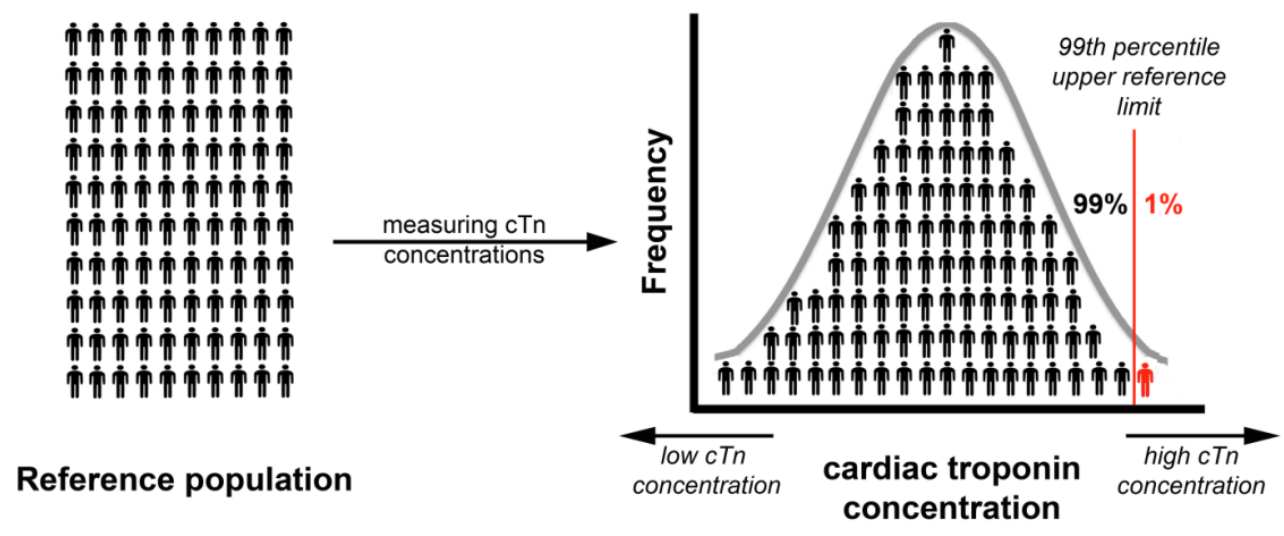

FIGURE 5. Illustration of the 99th percentile cTn concentration of a reference population of healthy individuals, used as the diagnostic cutoff for AMI.

\section{The era of high-sensitivity cardiac troponin assays}

At the time that the $99^{\text {th }}$ percentile cutoff was defined, none of the commercially available cTn assays were able to measure this concentration with sufficient precision (<10\% CV) [32, 34]. Ongoing improvements in technology over the past several years have advanced the analytical detection of cTn in the blood, permitting the detection of very low circulating levels of cTn with a higher precision [35-37]. The increased assay sensitivity is achieved by several modifications of the existing immunoassays. For instance, the hs-cTnT assay (Roche Diagnostics) requires a larger sample volume than the previous assay (50 $\mu \mathrm{L}$ instead of $15 \mu \mathrm{L}$ ). Moreover, signal amplification is obtained by the use of highly optimized antibody-ruthenium conjugates and interference by heterophilic antibodies has been reduced by using a chimeric mouse-human antibody as the detector antibody [34]. 
The introduction of hs-cTn concentrations resulted in an earlier detection of AMI. Several large clinical trials have demonstrated that the high-sensitivity assays have superior diagnostic performance in patients with suspected acute coronary syndromes in comparison to the contemporary assays [38-41]. Improved sensitivities have been reported for the hs-cTnT assay of Roche Diagnostics (from $83 \%$ to $95 \%$ ) [40] and hs-cTnl assay of Abbott Diagnostics (from $79 \%$ to 82\%) [39] (TABLE 1). Overall, this has enabled the earlier detection of the onset of AMI [40-42].

However, increased analytical sensitivity has come at the cost of decreased specificity (TABLE 1) and thus presenting additional diagnostic challenges for clinicians. Though cTn elevations signifies damage to the myocardium, cTn is not specific for one particular cardiovascular disease, such as AMI. Any condition that causes injury to the cardiomyocytes can result into increases in circulating hs-cTn concentrations, for example heart failure and myocarditis [44]. Furthermore, hs-cTn also accumulates in patients with chronic kidney disease [45], including end-stage renal disease (ESRD) [46, 47] but are usually stable and modest in such cases. The exact mechanisms that lead to these elevated cTn concentrations are not yet known, but it seems likely that a decrease in renal clearance contributes to this. In principle, cTnT and cTnl (intact or fragments) are small enough to be filtered by the kidneys [48]. Nevertheless, also in these patients, baseline hs-cTn concentrations remain strongly associated with increased cardiovascular risk and mortality [49-51].

Acute processes typically manifest with a rising and/or falling pattern, whereas chronic diseases generally do not. Subsequently, serial hs-cTn testing has demonstrated promising results in distinguishing acute from chronic disease $[39,52,53]$ by assessing absolute or relative changes, though absolute changes appear to be superior especially in the lower measuring range $[54,55]$. Many different absolute (5-7 ng/L) and relative (20-50\%) deltas have been reported, and increasingly earlier time-frames for monitoring is currently suggested (3-6 versus 1-2 hours) [54, 56]. 


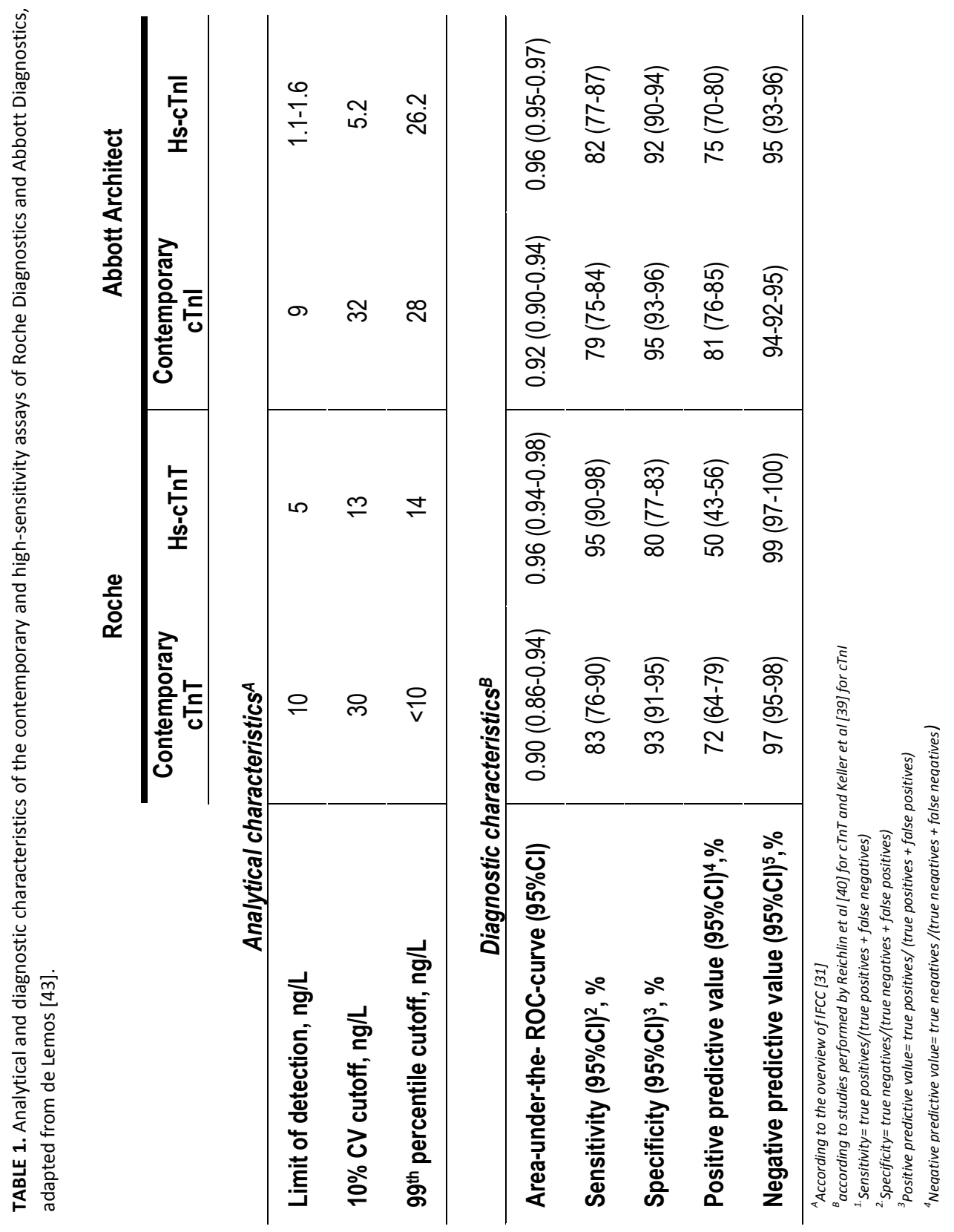




\section{Low levels of hs-cTn concentrations as a risk stratifying tool}

Hs-cTn assays are capable of measuring hs-cTn concentrations in most healthy reference subjects [35-37]. Several large clinical trials have demonstrated that low hs-cTnT [57-60] and hs-cTnl [61-64] concentrations, even below the diagnostic cut-off, are associated with the presence and severity of a wide range of cardiac abnormalities, including CAD [65, 66], cardiac morphology and function $[67,68]$. Also, the association of hs-cTn concentrations with a higher risk on developing cardiovascular events and all-cause mortality has been wellestablished in numerous populations [57-64, 69], from general cohorts [68] to stable chest pain patients [62]. Even when adjusted for traditional cardiovascular risk factors such as smoking, diabetes mellitus and hypercholesterolemia, hs-cTn concentrations remain important risk predictors. However, whether all detectable hs-cTn concentrations represents myocardial necrosis, remains to be elucidated. As such, other mechanisms have been proposed for these low levels of circulating cTn [70], for instance physiological renewal or remodelling of the heart [71], and cTn leakage from reversibly damaged cardiomyocytes during transient ischemia [72, 73]. 


\section{Thesis outline}

With the introduction of more sensitive cardiac troponin assays in the clinic, clinicians are often challenged when interpreting troponin values, especially in the lower measuring range. This thesis will shed light on important questions concerning the high-sensitivity assays and offers insight in the underlying reasons for hs-cTn elevations.

An overview of $99^{\text {th }}$ percentile upper reference limits and underlying reference populations is given in chapter two. In this chapter the challenges of defining a "cardio-healthy" reference population are discussed.

The interpretation of baseline hs-cTn values in the individual patient is complicated not only by cardiac disease but also renal dysfunction. As of yet it is unknown to what extent renal function contributes to higher cardiac troponin concentrations in patients with chest discomfort, in whom circulating hs-cTnT concentrations are mainly attributed to the presence and severity of atherosclerotic plaques or echocardiographic abnormalities. In-depth understanding of the relative contribution of renal function, next to cardiovascular disease, is examined in chapter three. In line with this, chapter four describes the effect of heart failure and renal dysfunction on hs-cTn concentrations, measured in an aged population (>65 years) of nursing home residents.

ESRD patients often exhibit elevated cTn levels that are more stable over time. To date, it remains unclear how dialysis affects cTn results, as both intradialytic increases and decreases have been reported. In chapter five hs-cTn concentrations before and after different modalities of hemodialysis are therefore investigated.

There is evidence that cTn proteins can be subjected to posttranslational modifications, such as degradation. Which circulating cTnT forms are detected by the clinical assay in the blood of AMI and ESRD patients are investigated in chapter six and chapter seven, respectively. 


\section{References}

1. Hamm, C.W., J.P. Bassand, S. Agewall, et al., ESC Guidelines for the management of acute coronary syndromes in patients presenting without persistent ST-segment elevation: The Task Force for the management of acute coronary syndromes (ACS) in patients presenting without persistent ST-segment elevation of the European Society of Cardiology (ESC). Eur Heart J, 2011. 32(23): p. 2999-3054.

2. Yeh, R.W., S. Sidney, M. Chandra, et al., Population trends in the incidence and outcomes of acute myocardial infarction. N Engl J Med, 2010. 362(23): p. 2155-65.

3. Vaartjes, I., C. Koopman, and I. van Dis, Hart- en vaatziekten in Nederland 2013, cijfers over leefstijl, risicofactoren, ziekte en sterfte. The Hague: Dutch Heart Foundation, 2013.

4. Thygesen, K., J.S. Alpert, A.S. Jaffe, et al., Third universal definition of myocardial infarction. Eur Heart J, 2012. 33(20): p. 2551-67.

5. Clerico, A., A. Giannoni, C. Prontera, et al., High-sensitivity troponin: a new tool for pathophysiological investigation and clinical practice. Adv Clin Chem, 2009. 49: p. 1-30.

6. Katus, H.A., A. Remppis, F.J. Neumann, et al., Diagnostic efficiency of troponin T measurements in acute myocardial infarction. Circulation, 1991. 83(3): p. 902-12.

7. Katus, H.A., S. Looser, K. Hallermayer, et al., Development and in vitro characterization of a new immunoassay of cardiac troponin T. Clin Chem, 1992. 38(3): p. 386-93.

8. Bodor, G.S., S. Porter, Y. Landt, et al., Development of monoclonal antibodies for an assay of cardiac troponin-I and preliminary results in suspected cases of myocardial infarction. Clin Chem, 1992. 38(11): p. 2203-14.

9. Takeda, S., A. Yamashita, K. Maeda, et al., Structure of the core domain of human cardiac troponin in the $\mathrm{Ca}(2+)$-saturated form. Nature, 2003. 424(6944): p. 35-41.

10. Thygesen, K., J. Mair, H. Katus, et al., Recommendations for the use of cardiac troponin measurement in acute cardiac care. Eur Heart J, 2010. 31(18): p. 2197-204.

11. Katus, H.A., A. Remppis, T. Scheffold, et al., Intracellular compartmentation of cardiac troponin $\mathrm{T}$ and its release kinetics in patients with reperfused and nonreperfused myocardial infarction. Am J Cardiol, 1991. 67(16): p. 1360-7.

12. Remppis, A., T. Scheffold, J. Greten, et al., Intracellular compartmentation of troponin T: release kinetics after global ischemia and calcium paradox in the isolated perfused rat heart. J Mol Cell Cardiol, 1995. 27(2): p. 793-803.

13. Bleier, J., K.P. Vorderwinkler, J. Falkensammer, et al., Different intracellular compartmentations of cardiac troponins and myosin heavy chains: a causal connection to their different early release after myocardial damage. Clin Chem, 1998. 44(9): p. 1912-8.

14. Starnberg, K., A. Jeppsson, B. Lindahl, et al., Revision of the troponin T release mechanism from damaged human myocardium. Clin Chem, 2014. 60(8): p. 1098-104.

15. Jeong, E.M., X. Wang, K. Xu, et al., Nonmyofilament-associated troponin T fragments induce apoptosis. Am J Physiol Heart Circ Physiol, 2009. 297(1): p. H283-92.

16. Michielsen, E.C., J.H. Diris, V.W. Kleijnen, et al., Investigation of release and degradation of cardiac troponin $T$ in patients with acute myocardial infarction. Clin Biochem, 2007. 40(12): p. 851-5.

17. Solecki, K., A.M. Dupuy, N. Kuster, et al., Kinetics of high-sensitivity cardiac troponin T or troponin I compared to creatine kinase in patients with revascularized acute myocardial infarction. Clin Chem Lab Med, 2014.

18. Streng, A.S., D. de Boer, J. van der Velden, et al., Posttranslational modifications of cardiac troponin T: an overview. J Mol Cell Cardiol, 2013. 63: p. 47-56.

19. Peronnet, E., L. Becquart, F. Poirier, et al., SELDI-TOF MS analysis of the Cardiac Troponin I forms present in plasma from patients with myocardial infarction. Proteomics, 2006. 6(23): p. 6288-99.

20. Wu, A.H., Y.J. Feng, R. Moore, et al., Characterization of cardiac troponin subunit release into serum after acute myocardial infarction and comparison of assays for troponin $\mathrm{T}$ and $\mathrm{I}$. 
American Association for Clinical Chemistry Subcommittee on cTnl Standardization. Clin Chem, 1998. 44(6 Pt 1): p. 1198-208.

21. Katrukha, A.G., A.V. Bereznikova, T.V. Esakova, et al., Troponin I is released in bloodstream of patients with acute myocardial infarction not in free form but as complex. Clin Chem, 1997. 43(8 Pt 1): p. 1379-85.

22. Labugger, R., L. Organ, C. Collier, et al., Extensive troponin I and T modification detected in serum from patients with acute myocardial infarction. Circulation, 2000. 102(11): p. 1221-6.

23. McDonough, J.L., D.K. Arrell, and J.E. Van Eyk, Troponin I degradation and covalent complex formation accompanies myocardial ischemia/reperfusion injury. Circ Res, 1999. 84(1): p. 920.

24. Bates, K.J., E.M. Hall, M.N. Fahie-Wilson, et al., Circulating immunoreactive cardiac troponin forms determined by gel filtration chromatography after acute myocardial infarction. Clin Chem, 2010. 56(6): p. 952-8.

25. Alpert, J.S., K. Thygesen, E. Antman, et al., Myocardial infarction redefined--a consensus document of The Joint European Society of Cardiology/American College of Cardiology Committee for the redefinition of myocardial infarction. J Am Coll Cardiol, 2000. 36(3): p. 959-69.

26. Thygesen, K., J.S. Alpert, H.D. White, et al., Universal definition of myocardial infarction. Eur Heart J, 2007. 28(20): p. 2525-38.

27. Apple, F.S., R.L. Jesse, L.K. Newby, et al., National Academy of Clinical Biochemistry and IFCC Committee for Standardization of Markers of Cardiac Damage Laboratory Medicine Practice Guidelines: analytical issues for biochemical markers of acute coronary syndromes. Clin Chem, 2007. 53(4): p. 547-51.

28. Morrow, D.A., C.P. Cannon, R.L. Jesse, et al., National Academy of Clinical Biochemistry Laboratory Medicine Practice Guidelines: clinical characteristics and utilization of biochemical markers in acute coronary syndromes. Clin Chem, 2007. 53(4): p. 552-74.

29. Keller, T., T. Zeller, D. Peetz, et al., Sensitive troponin I assay in early diagnosis of acute myocardial infarction. N Engl J Med, 2009. 361(9): p. 868-77.

30. Apple, F.S., R.L. Jesse, L.K. Newby, et al., National Academy of Clinical Biochemistry and IFCC Committee for Standardization of Markers of Cardiac Damage Laboratory Medicine Practice Guidelines: Analytical issues for biochemical markers of acute coronary syndromes. Circulation, 2007. 115(13): p. e352-5.

31. Apple, F.S., P.O. Collinson, and I.T.F.o.C.A.o.C. Biomarkers, Analytical characteristics of highsensitivity cardiac troponin assays. Clin Chem, 2012. 58(1): p. 54-61.

32. Apple, F.S., A new season for cardiac troponin assays: it's time to keep a scorecard. Clin Chem, 2009. 55(7): p. 1303-6.

33. Apple, F.S., R. Ler, and M.M. Murakami, Determination of 19 cardiac troponin I and T assay 99th percentile values from a common presumably healthy population. Clin Chem, 2012. 58(11): p. 1574-81.

34. Panteghini, M., F. Pagani, K.T. Yeo, et al., Evaluation of imprecision for cardiac troponin assays at low-range concentrations. Clin Chem, 2004. 50(2): p. 327-32.

35. Mingels, A., L. Jacobs, E. Michielsen, et al., Reference population and marathon runner sera assessed by highly sensitive cardiac troponin $\mathrm{T}$ and commercial cardiac troponin $\mathrm{T}$ and I assays. Clin Chem, 2009. 55(1): p. 101-8.

36. McKie, P.M., D.M. Heublein, C.G. Scott, et al., Defining high-sensitivity cardiac troponin concentrations in the community. Clin Chem, 2013. 59(7): p. 1099-107.

37. Krintus, M., M. Kozinski, P. Boudry, et al., European multicenter analytical evaluation of the Abbott ARCHITECT STAT high sensitive troponin I immunoassay. Clin Chem Lab Med, 2014. 52(11): p. 1657-65.

38. Giannitsis, E., M. Becker, K. Kurz, et al., High-sensitivity cardiac troponin T for early prediction of evolving non-ST-segment elevation myocardial infarction in patients with suspected acute coronary syndrome and negative troponin results on admission. Clin Chem, 2010. 56(4): p. 642-50. 
39. Keller, T., T. Zeller, F. Ojeda, et al., Serial changes in highly sensitive troponin I assay and early diagnosis of myocardial infarction. JAMA, 2011. 306(24): p. 2684-93.

40. Reichlin, T., W. Hochholzer, S. Bassetti, et al., Early diagnosis of myocardial infarction with sensitive cardiac troponin assays. N Engl J Med, 2009. 361(9): p. 858-67.

41. Reiter, M., R. Twerenbold, T. Reichlin, et al., Early diagnosis of acute myocardial infarction in patients with pre-existing coronary artery disease using more sensitive cardiac troponin assays. Eur Heart J, 2012. 33(8): p. 988-97.

42. Body, R., S. Carley, G. McDowell, et al., Rapid exclusion of acute myocardial infarction in patients with undetectable troponin using a high-sensitivity assay. J Am Coll Cardiol, 2011. 58(13): p. 1332-9.

43. de Lemos, J.A., Increasingly sensitive assays for cardiac troponins: a review. JAMA, 2013. 309(21): p. 2262-9.

44. Newby, L.K., R.L. Jesse, J.D. Babb, et al., ACCF 2012 expert consensus document on practical clinical considerations in the interpretation of troponin elevations: a report of the American College of Cardiology Foundation task force on Clinical Expert Consensus Documents. J Am Coll Cardiol, 2012. 60(23): p. 2427-63.

45. deFilippi, C., S.L. Seliger, W. Kelley, et al., Interpreting cardiac troponin results from highsensitivity assays in chronic kidney disease without acute coronary syndrome. Clin Chem, 2012. 58(9): p. 1342-51.

46. Jacobs, L.H., J. van de Kerkhof, A.M. Mingels, et al., Haemodialysis patients longitudinally assessed by highly sensitive cardiac troponin $\mathrm{T}$ and commercial cardiac troponin $\mathrm{T}$ and cardiac troponin I assays. Ann Clin Biochem, 2009. 46(Pt 4): p. 283-90.

47. Aakre, K.M., T. Roraas, P.H. Petersen, et al., Weekly and 90-minute biological variations in cardiac troponin $\mathrm{T}$ and cardiac troponin I in hemodialysis patients and healthy controls. Clin Chem, 2014. 60(6): p. 838-47.

48. Diris, J.H., C.M. Hackeng, J.P. Kooman, et al., Impaired renal clearance explains elevated troponin T fragments in hemodialysis patients. Circulation, 2004. 109(1): p. 23-5.

49. Gravning, J., E.T. Askevold, S.H. Nymo, et al., Prognostic effect of high-sensitive troponin T assessment in elderly patients with chronic heart failure: results from the CORONA trial. Circ Heart Fail, 2014. 7(1): p. 96-103.

50. Miller, W.L., K.A. Hartman, M.F. Burritt, et al., Profiles of serial changes in cardiac troponin T concentrations and outcome in ambulatory patients with chronic heart failure. J Am Coll Cardiol, 2009. 54(18): p. 1715-21.

51. Januzzi, J.L., Jr., G. Filippatos, M. Nieminen, et al., Troponin elevation in patients with heart failure: on behalf of the third Universal Definition of Myocardial Infarction Global Task Force: Heart Failure Section. Eur Heart J, 2012. 33(18): p. 2265-71.

52. Mueller, M., M. Biener, M. Vafaie, et al., Absolute and relative kinetic changes of highsensitivity cardiac troponin $\mathrm{T}$ in acute coronary syndrome and in patients with increased troponin in the absence of acute coronary syndrome. Clin Chem, 2012. 58(1): p. 209-18.

53. Giannitsis, E. and H.A. Katus, Cardiac troponin level elevations not related to acute coronary syndromes. Nat Rev Cardiol, 2013. 10(11): p. 623-34.

54. Reichlin, T., A. Irfan, R. Twerenbold, et al., Utility of absolute and relative changes in cardiac troponin concentrations in the early diagnosis of acute myocardial infarction. Circulation, 2011. 124(2): p. 136-45.

55. Haaf, P., B. Drexler, T. Reichlin, et al., High-sensitivity cardiac troponin in the distinction of acute myocardial infarction from acute cardiac noncoronary artery disease. Circulation, 2012. 126(1): p. 31-40.

56. Aldous, S.J., A.M. Richards, L. Cullen, et al., Early dynamic change in high-sensitivity cardiac troponin $T$ in the investigation of acute myocardial infarction. Clin Chem, 2011. 57(8): p. 1154-60.

57. Everett, B.M., N.R. Cook, M.C. Magnone, et al., Sensitive cardiac troponin T assay and the risk of incident cardiovascular disease in women with and without diabetes mellitus: the Women's Health Study. Circulation, 2011. 123(24): p. 2811-8. 
58. Masson, S., I. Anand, C. Favero, et al., Serial measurement of cardiac troponin T using a highly sensitive assay in patients with chronic heart failure: data from 2 large randomized clinical trials. Circulation, 2012. 125(2): p. 280-8.

59. Saunders, J.T., V. Nambi, J.A. de Lemos, et al., Cardiac troponin T measured by a highly sensitive assay predicts coronary heart disease, heart failure, and mortality in the Atherosclerosis Risk in Communities Study. Circulation, 2011. 123(13): p. 1367-76.

60. deFilippi, C.R., J.A. de Lemos, R.H. Christenson, et al., Association of serial measures of cardiac troponin $\mathrm{T}$ using a sensitive assay with incident heart failure and cardiovascular mortality in older adults. JAMA, 2010. 304(22): p. 2494-502.

61. Zeller, T., H. Tunstall-Pedoe, O. Saarela, et al., High population prevalence of cardiac troponin I measured by a high-sensitivity assay and cardiovascular risk estimation: the MORGAM Biomarker Project Scottish Cohort. Eur Heart J, 2014. 35(5): p. 271-81.

62. Omland, T., M.A. Pfeffer, S.D. Solomon, et al., Prognostic value of cardiac troponin I measured with a highly sensitive assay in patients with stable coronary artery disease. J Am Coll Cardiol, 2013. 61(12): p. 1240-9.

63. Mills, N.L., A.M. Churchhouse, K.K. Lee, et al., Implementation of a sensitive troponin I assay and risk of recurrent myocardial infarction and death in patients with suspected acute coronary syndrome. JAMA, 2011. 305(12): p. 1210-6.

64. Eggers, K.M., P. Venge, B. Lindahl, et al., Cardiac troponin I levels measured with a highsensitive assay increase over time and are strong predictors of mortality in an elderly population. J Am Coll Cardiol, 2013. 61(18): p. 1906-13.

65. Laufer, E.M., A.M. Mingels, M.H. Winkens, et al., The extent of coronary atherosclerosis is associated with increasing circulating levels of high sensitive cardiac troponin T. Arterioscler Thromb Vasc Biol, 2010. 30(6): p. 1269-75.

66. Januzzi, J.L., Jr., F. Bamberg, H. Lee, et al., High-sensitivity troponin T concentrations in acute chest pain patients evaluated with cardiac computed tomography. Circulation, 2010. 121(10): p. 1227-34.

67. Chin, C.W., A.S. Shah, D.A. McAllister, et al., High-sensitivity troponin I concentrations are a marker of an advanced hypertrophic response and adverse outcomes in patients with aortic stenosis. Eur Heart J, 2014. 35(34): p. 2312-21.

68. de Lemos, J.A., M.H. Drazner, T. Omland, et al., Association of troponin T detected with a highly sensitive assay and cardiac structure and mortality risk in the general population. JAMA, 2010. 304(22): p. 2503-12.

69. Mingels, A.M., I.A. Joosen, M.O. Versteylen, et al., High-sensitivity cardiac troponin T: risk stratification tool in patients with symptoms of chest discomfort. PLoS One, 2012. 7(4): p. e35059.

70. Giannoni, A., S. Giovannini, and A. Clerico, Measurement of circulating concentrations of cardiac troponin $\mathrm{I}$ and $\mathrm{T}$ in healthy subjects: a tool for monitoring myocardial tissue renewal? Clin Chem Lab Med, 2009. 47(10): p. 1167-77.

71. Bergmann, O., R.D. Bhardwaj, S. Bernard, et al., Evidence for cardiomyocyte renewal in humans. Science, 2009. 324(5923): p. 98-102.

72. Hessel, M.H., E.C. Michielsen, D.E. Atsma, et al., Release kinetics of intact and degraded troponin I and T after irreversible cell damage. Exp Mol Pathol, 2008. 85(2): p. 90-5.

73. Hickman, P.E., J.M. Potter, C. Aroney, et al., Cardiac troponin may be released by ischemia alone, without necrosis. Clin Chim Acta, 2010. 411(5-6): p. 318-23. 




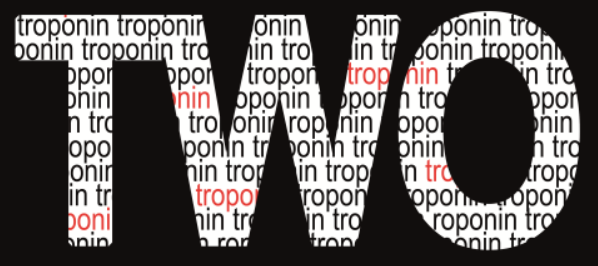

A COMPREHENSIVE REVIEW OF UPPER REFERENCE LIMITS REPORTED FOR (HIGH-) SENSITIVITY CARDIAC TROPONIN ASSAYS: THE CHALLENGES THAT LIE AHEAD

Cardinaels E, Mingels A, Jacobs L,Meex S, Bekers $O$ and van Dieijen-Visser M.

Clin Chem Lab Med 2012, 50 (5), 791-806 


\section{Abstract}

Cardiac troponins (cTn) are the preferred markers for the diagnosis of acute myocardial infarction (AMI). The guidelines recommend the use of the $99^{\text {th }}$ percentile upper reference concentration of a healthy population as the diagnostic cutoff for AMI. However, a broad range of upper reference limits is still employed, complicating the diagnosis of AMI.

This overview is meant to assist laboratory specialists to define an appropriate cutoff value for the diagnosis of AMI. Therefore we provide an overview of the analytical performance and upper reference limits of seven (high-)sensitivity cTn assays: Roche high-sensitivity cTnT and ADVIA Centaur, Stratus CS, Dimension Vista, Vitros ECi, Access and Architect cTnl assays.

It is shown that none of the reference populations completely met the guidelines, including those in package inserts. $40 \%$ of the studies collected less than the advised minimum of 300 subjects. Many studies (50\%) did not report their inclusion criteria, while lower $99^{\text {th }}$ percentile limits were observed when more stringent selection criteria were applied. Higher troponin cutoffs were found in men and elderly subjects, suggesting gender- and age-specific cutoffs would be considered. Therefore, there is still need for a large, rigorously screened reference population to more accurately establish cTn upper reference limits.

List of Abbreviations: cTn, cardiac troponin; ACS, acute coronary syndrome; NACB, National Academy for Clinical Biochemistry; ESC, European Society of Cardiology; ACC, American College of Cardiology; AMI, acute myocardial infarction; $c T n T$, cardiac troponin T; cTnl, cardiac troponin I; CV, coefficient of variation; $h s-c T n T$, high-sensitivity $C T n T$; $L O B$, limit of blank; $L O D$, limit of detection; $R C V$, reference change value; $C V_{A}$, analytical coefficient of variation; $C V_{l}$, within-subject coefficient of variation; II, index of individuality; $C V_{G}$, betweensubject coefficient of variation; IFCC, International Federation of Clinical Chemistry and Laboratory Medicine; 


\section{Introduction}

Cardiac troponins (cTn) were generally recommended as the biochemical gold standard for the investigation of patients with acute coronary syndrome (ACS) in 1999 by the National Academy of Clinical Biochemistry (NACB) [1], and in 2000 by the European Society of Cardiology and the American College of Cardiology (ESC/ACC) [2]. Both clinical [3] and analytical aspects [4, 5] of these guidelines were revised in 2007, in order to establish a more universal definition of acute myocardial infarction (AMI). According to these guidelines a rise and/or fall of cardiac troponin $\mathrm{T}$ (cTnT) or I (cTnl) with at least one value above the 99th percentile of a healthy reference population is the hallmark of myocardial damage. To complete the diagnosis, cTn changes must be accompanied with at least one of the following clinical factors: the presence of ischemic symptoms, electrocardiographic (ECG) alterations (for example ST-T changes, left bundle branch block or development of pathological $Q$ waves), or imaging evidence of either new loss of viable myocardium or new regional wall motion abnormality $[3,6]$. Importantly, the guidelines recommend a total imprecision (CV) of $10 \%$ at the 99th percentile reference limit [5]. In a recent overview, Apple classified the available assays [7] as "not acceptable" (CV >20\%), "clinically usable" (CV 10$20 \%$ ) or "guideline acceptable" (CV $\leq 10 \%$ ) [8]. In addition, assays were categorized into 4 levels depending on whether $<50 \%$ (level 1), 50-75\% (level 2), $75-95 \%$ (level 3) or $>95 \%$ (level 4) of reference concentrations had detectable cTn concentrations below the 99th percentile. Only nine out of twenty-two assays were labeled "guideline acceptable" and nine were defined as "clinically usable" [8]. However, as recently pointed out, misclassification of patients when using CVs between $10 \%$ and $20 \%$ at the 99 th percentile is considered to be insignificant [9].

Despite the introduction of guideline acceptable assays and the recommendation to use the 99th percentile value of a reference population as cutoff for AMI, a broad range of cutoff concentrations are in use, complicating the diagnosis of AMI for clinicians. A major cause of this heterogeneity is lack of assay harmonization and non-standardized selection of healthy reference populations [10-12]. This issue will become even more prominent with the ongoing development of increasingly sensitive assays that detect measurable 
cTn levels in almost everybody. Noteworthy, recent data indicate that even low levels of cTn can predict major adverse cardiac events, pointing out the importance of reliable diagnostic cutoffs $[13,14]$.

Most laboratories do not have the resources to determine their own 99th percentile cutoff. For appropriate statistical determination of the 99th percentile cutoff a theoretical minimum of 120 reference subjects has been proposed [15]. In order to reliably assess the 99 th percentile with an uncertainty $<5 \%$, a sample size of 300 subjects is required $[5,6]$. Furthermore, the distribution of gender and age in the reference population should resemble those in a typical group of AMI patients, and should ideally have negative exercise stress tests and normal cardiac function as assessed by non-invasive imaging. The CARMAGUE study pointed out that $\pm 50 \%$ of the laboratories employ cutoff values from the package insert, 15\% adopt values from peer-reviewed studies [16], and sometimes arbitrarily chosen values are used. A structured overview of all studies may be a helpful tool that facilitates the diagnostic process and assists laboratories to define appropriate cutoff values.

In the present review, we provide an extensive summary of seven cTn assays, frequently used in clinical practice, that can measure the 99th percentile cutoff with a high precision, including five "guideline acceptable" assays: 1) high sensitivity cTnT (hs-cTnT, Roche Diagnostics), 2) ADVIA Centaur Tnl-Ultra (Siemens Healthcare Diagnostics), 3) Stratus CS Acute Care cTnl (Siemens Healthcare Diagnostics), 4) Dimension Vista cTnl (Siemens Healthcare Diagnostics) and 5) Vitros ECi cTnl ES (Ortho Clinical Diagnostics) assays. We also included two "clinically usable" assays to complete the overview: 6) Access AccuTnl (Beckman Coulter) and 7) Architect cTnl assay (Abbott Diagnostics). Point-of-care tests and research assays were excluded. Between October 2010 and October 2011, we searched the MEDLINE electronic database for publications in English, using the key words "troponin", "troponin assay", and "99th percentile". We paid special attention to analytical assay characteristics and the 99th percentile cutoffs. We also discuss selection criteria of the reference population, gender/age specific differences in cTn concentrations, and biological variation within or between subjects. These are increasingly 
prominent subjects of debate since the introduction of the high sensitivity assays.

\section{Hs-cTnT Assay, Roche Diagnostics}

The analytical evaluation of the assay established by the manufacturer (Roche Diagnostics, Mannheim, Germany) described a limit of blank (LoB) of $3 \mathrm{ng} / \mathrm{L}$ and limit of detection (LoD) of $5 \mathrm{ng} / \mathrm{L}$. As depicted in TABLE 1A, other studies reported LoDs that ranged from 1 to $5 \mathrm{ng} / \mathrm{L}$ [17-21]. According to the package insert a $10 \% \mathrm{CV}$ was measured at $13 \mathrm{ng} / \mathrm{L}$ (version 2010). Most authors found the same value or even lower $10 \%$ CV cutoffs [18, 22-26]. Only one study reports a noticeable higher $10 \% \mathrm{CV}$ of $18.5 \mathrm{ng} / \mathrm{L}$ [27]. These discrepancies in LoB, LoD, and $10 \% \mathrm{CV}$ can be the result of using various methods of estimation [11].

The reference populations were selected by either health questionnaires [21], laboratory tests $[17,22]$, or both approaches in combination with echocardiography $[25,27]$. In most studies inclusion criteria were not described $[18-20,23,24]$. The recommended inclusion of at least 120 cardio-healthy reference subjects, required for reliable estimation of the $99^{\text {th }}$ percentile, was met in most studies, except in Koerbin et al and Vasatova et al using 104 and 73 subjects, respectively $[19,25]$.

The $99^{\text {th }}$ percentile concentration according to the package insert was $14 \mathrm{ng} / \mathrm{L}$ and calculated from 533 healthy subjects. This value was reproduced in four independent studies $[18,20,21,24]$. However, in literature, slightly lower $99^{\text {th }}$ percentile limits $( \pm 12 \mathrm{ng} / \mathrm{L})$ were described in a large reference cohort $(n=1061)$ of healthy subjects using EDTA plasma samples [17] or a small population of 104 subjects, broadly screened by means of questionnaires to exclude possible risk factors, presence of cardiac diseases and cardiovascular medications, followed by stress echocardiography and routine chemistry tests [25]. In our lab, a cutoff of $16 \mathrm{ng} / \mathrm{L}$ was found [22] and similar $99^{\text {th }}$ percentile cutoffs were also reported by others, using smaller reference populations [26], comprised of $66 \%$ males [19].

Gender related differences in cTnT levels have been documented using the hscTnT assay [18, 21, 22, 24, 25, 27]. All studies except one [27] reported higher 
cTnT concentrations in males $(P<0.01)$. From the reported concentrations in TABLE 1, we calculated a mean $99^{\text {th }}$ percentile cutoff of $15.0 \mathrm{ng} / \mathrm{L}$ for males and $9.6 \mathrm{ng} / \mathrm{L}$ for females $[21,22,24,25]$. It has been suggested that this could be due to gender specific differences in the pathophysiology of ACS [21], or variations in mean heart sizes between men and women [22]. Several studies indicated higher cTnT levels in elderly [18, 22, 25]. Significantly higher cTnT levels were found in people over 60 years, compared to younger subjects, both in the male and female cohort $(P<0.01)$ [25]. These findings provide an independent replication of a previous report [28].

Taken together, the $99^{\text {th }}$ percentile cutoff varied between 12 to $17 \mathrm{ng} / \mathrm{L}$. Moreover, in all studies but one, the imprecision was $\leq 10 \%$.

\section{Architect cTnl assay (Abbott Diagnostics)}

The lowest cTn concentration that can be measured with an analytical imprecision of $10 \%$ was reported to be $32 \mathrm{ng} / \mathrm{L}$ by the manufacturer (Abbott Diagnostics, Abbott Park, IL, USA) and others [22, 29-31] (TABLE 1B). In the remaining publications, the $10 \% \mathrm{CV}$ values were indicated to be higher, ranging from $40 \mathrm{ng} / \mathrm{L}$ to $75 \mathrm{ng} / \mathrm{L}$ [32-35] .

The screening methods for the reference populations were primarily not specified [30-32, 36]. For this assay, only three reference populations were screened by means of medical history check, ECG and blood laboratory tests $[29,34,35]$. One study included less than the advised minimum of 300 subjects [34]. The reference population, used by the manufacturer, consisted of 224 apparently healthy subjects.

For the $99^{\text {th }}$ percentiles cutoff a concentration of $28 \mathrm{ng} / \mathrm{L}$ was found by the manufacturer and many others report similar results [30, 31, 33, 36]. Six independent studies, report cutoffs lower than $20 \mathrm{ng} / \mathrm{L}$ [22, 32, 34-36]. Only one study reported noticeably higher $99^{\text {th }}$ percentile cutoffs in a reference population of 442 subjects, screened by means of medical history check, ECG and routine blood analysis [29].

Age and gender related differences in $99^{\text {th }}$ percentile cutoffs were not statistically significant among these studies. 
In summary, upper reference limits varied between 13 and $30 \mathrm{ng} / \mathrm{L}$, while for the $10 \%$ CV cutoff, values between 30 and $76 \mathrm{ng} / \mathrm{L}$ were reported.

\section{Access AccuTnl assay (Beckman Coulter)}

Evaluation of the analytical sensitivity by the manufacturer (Beckman Coulter, Woerden, the Netherlands) indicated a LoD of $4 \mathrm{ng} / \mathrm{L}$ according to the package insert (version 2006). $60 \mathrm{ng} / \mathrm{L}$ is the lowest $\mathrm{cTnl}$ concentration which can be measured with a $10 \% \mathrm{CV}$. Various studies reported similar [37-39] or somewhat higher values: $104 \mathrm{ng} / \mathrm{L}$ [34]; 101 [35] and 75 [40]. 20\% CVs were obtained at 43 $\mathrm{ng} / \mathrm{L}, 34 \mathrm{ng} / \mathrm{L}$ and $15 \mathrm{ng} / \mathrm{L}$ respectively (TABLE 1C). Recently, the AccuTnl assay was optimized resulting in a lower $10 \%$ CV value of $14 \mathrm{ng} / \mathrm{L}[41,42]$.

As described previously, the reference populations consisted mostly of apparently healthy subjects, with no details on how the subjects were screened $[36,38,39,43-45]$. In the various studies, health questionnaires were applied either alone [35, 46], or in combination with ECG and routine blood analysis [29, 34, 40, 42, 47]. Eggers et al clearly showed that a lower cutoff was found when screening for cardiovascular diseases $(28 \mathrm{ng} / \mathrm{L})$ in comparison to the unscreened reference population (44 ng/L) [41].

Similar $99^{\text {th }}$ percentile cutoff as indicated by the package insert ( $\left.40 \mathrm{ng} / \mathrm{L}\right)$ were mostly described using a wide variety of reference populations [29, 34, 36, 37, $39,44,45,47]$. Lower cutoffs were found when less than 300 subjects were included $[38,43,48]$ or in a relatively young population [35]. On the other hand, higher $99^{\text {th }}$ percentile concentrations $(60-80 \mathrm{ng} / \mathrm{L})$ were also measured $[40,42]$. A sample matrix effect was observed between serum and plasma [40], although others opposed this finding [29, 47, 48].

Gender-related differences in the $99^{\text {th }}$ percentile cutoffs were reported to be statistically significant in three studies [41, 44, 46]. Ethnicity was also reported to affect the $99^{\text {th }}$ percentile cutoff [46], although later on this observation was contradicted in another study [44].

Conclusion. Wide variations in $10 \% \mathrm{CV}$ values and $99^{\text {th }}$ cutoffs are observed ranging from 14-104 $\mathrm{ng} / \mathrm{L}$ and $13-80 \mathrm{ng} / \mathrm{L}$ respectively. In some but not all studies the $99^{\text {th }}$ percentile was measured with a $C V<10 \%$, indicating guideline 
acceptance. However, many reported cutoffs were not in agreement with the manufacturer's statements.

\section{ADVIA Centaur TnI-Ultra Assay, Siemens Healthcare Diagnostics}

Analytical sensitivity. Four studies with the new generation ADVIA centaur reported a LoD of $6 \mathrm{ng} / \mathrm{L}[35,49-51]$, and one study found a slightly higher LoD of $8.5 \mathrm{ng} / \mathrm{L}$ [52]. The package insert (Siemens Healthcare Diagnostics, Frimley, Camberley, UK, version 2009) stated that the LoD of the ADVIA Centaur TnlUltra assay is $6 \mathrm{ng} / \mathrm{L}$ and $30 \mathrm{ng} / \mathrm{L}$ is the minimum concentration that could be measured with an analytical imprecision of $<10 \%$. This value was reproduced in four studies [35, 42, 51, 53], but higher $10 \%$ CV cutoffs ranging from $45 \mathrm{ng} / \mathrm{L}$ to $67 \mathrm{ng} / \mathrm{L}[34,49,50,52,54]$ were also reported (TABLE 1D).

A cardio-healthy reference population was selected by laboratory tests [49, 54], sometimes in combination with echocardiography [34, 42, 50, 55]. Three studies excluded cardiac diseases and their possible risk factors by means of medical history examinations and/or health questionnaires [35, 52, 53]. The majority of studies included $>300$ subjects. Only two studies included less than the recommended 300 subjects $[34,52]$ from which one study did not meet the minimum inclusion of 120 subjects [34].

The $99^{\text {th }}$ percentile cutoff measured by the manufacturer is $40 \mathrm{ng} / \mathrm{L}$, for both serum and plasma samples. Similar values were found in two studies $[50,51]$ but 1.5 to 2 fold higher $99^{\text {th }}$ percentile concentrations have also been reported for lithium heparin plasma and serum samples [42, 49, 52-55]. Venge et al attributed their higher $99^{\text {th }}$ percentile cutoff $(80 \mathrm{ng} / \mathrm{L})$ to different inclusion criteria of the reference population, or the use of samples that had been frozen for a substantial length of time [42]. It should be noted that an older population was used in this study. A majority of the subjects were male and three outliers were excluded with no reported reason. Tate et al included only 108 subjects in the reference population, rigorously screened for cardiovascular risk factors, cardiac diseases, cardiovascular medications, stress echocardiography, blood and urine routine tests and heterophilic antibodies against cTn, which could explain the relatively low $99^{\text {th }}$ percentile concentration of $21 \mathrm{ng} / \mathrm{L}$ found both in plasma and serum samples [34]. Even lower $99^{\text {th }}$ percentile cutoffs (13 ng/L and $18 \mathrm{ng} / \mathrm{L}$ for 
plasma and serum, respectively) were reported in a relatively young reference population with a mean age of 30 years [35].

Gender dependent differences were also reported, with significantly higher cTnl concentrations in men than in women $(P<0.05)[35,49,54,55]$. Statistically significant correlations between age and cTnl concentrations were reported $(R=0.268 ; P<0.0001)[49,54]$, and in line with this observation older subjects had higher cTnl levels than the younger subjects $(P \leq 0.0002)[42,55]$. No significant differences in $99^{\text {th }}$ percentile cutoffs were found among multi-ethnical Asian populations [53].

In conclusion, $10 \% \mathrm{CV}$ and $99^{\text {th }}$ percentile cutoffs ranged between $30-67 \mathrm{ng} / \mathrm{L}$ and $13-87 \mathrm{ng} / \mathrm{L}$, respectively. Most, but not all studies [34, 35, 50] concluded that the ADVIA Centaur Tnl-Ultra Assay is guideline acceptable.

\section{Stratus CS Acute Care cTnI Assay, Siemens Healthcare Diagnostics}

The analytical evaluation of the assay established by the manufacturer (Siemens Healthcare Diagnostics) yielded a LoB of $30 \mathrm{ng} / \mathrm{L}$ and a LoD of 60 $\mathrm{ng} / \mathrm{L}$, while other studies reported LoDs ranging from 10 to $20 \mathrm{ng} / \mathrm{L}$ [56-59]. According to the package insert $60 \mathrm{ng} / \mathrm{L}$ is the minimum concentration that can be measured with an analytical imprecision of $<10 \%$ (version 2001). In literature similar results were found [57-59] (TABLE 1E).

The reference population was not specified in three studies, including the package insert, $[57,58]$. One study screened for history of cardiac disease, hematochemical alterations and hyperpyrexia [59]. One study performed a history check, ECG, treadmill tests, stress echocardiography or angiogram [56]. Three studies, including those of the manufacturer, analyzed sample sizes that were smaller than the minimum number of 120 subjects [57, 59]. Only Christenson et al included more than the recommended 300 subjects [58].

The $99^{\text {th }}$ percentile concentration provided by the manufacturer is $70 \mathrm{ng} / \mathrm{L}$. This value was reproduced by Christenson [58], but lower cutoff concentrations of 30 $\mathrm{ng} / \mathrm{L}$ [59] and $50 \mathrm{ng} / \mathrm{L}$ [57] were also reported with an accompanying $10 \%$ $\mathrm{CV} / 99^{\text {th }}$ percentile ratio of 2.33 and 1.20 respectively. These lower estimations may be due to the inclusion of younger subjects, and the small sample size. 
In conclusion, only one reference population included a sufficiently large sample size and reported the assay to be guideline acceptable [58]. More studies are however needed for the Stratus CS assay to make valid conclusions.

\section{Dimension Vista cTnl Assay, Siemens Healthcare Diagnostics}

Analytical validation of the Dimension Vista cTnl assay by the manufacturer (Siemens Healthcare Diagnostics) yielded a LoB of $15 \mathrm{ng} / \mathrm{L}$ and a total imprecision (CV) below $10 \%$ at $40 \mathrm{ng} / \mathrm{L}$ (version 2008). Only one independent study validated the sensitivity of the Dimension Vista cTnl Assay, and reported a LoB, LoD and $10 \%$ CV cutoff of 11,15 , and $36 \mathrm{ng} / \mathrm{L}$, respectively [60].

The reference population collected by the manufacturer included 199 people with no clear description of the applied screening methods. Arrabola et al included 350 individuals ( $73 \%$ male) after examination of the patient's medical history [60] (TABLE 1F).

The $99^{\text {th }}$ percentile cutoff calculated by the manufacturer is $45 \mathrm{ng} / \mathrm{L}$. Arrabola et al reported a lower cutoff ( $22 \mathrm{ng} / \mathrm{L})$, and a $10 \% \mathrm{CV} / 99^{\text {th }}$ percentile ratio of 1.64 .

No significant gender and age related differences in cTnl levels are currently described for this assay. However, caution is required in interpretation of the limited available evidence [60].

In conclusion. Although more studies are required to confirm these results, the Dimension Vista cTnl Assay is not guideline acceptable according to the current evidence in literature [60].

\section{Vitros ECi Troponin I ES Assay, Ortho Clinical Diagnostics}

Analytical validation of the Vitros ECi cTnI ES assay by the manufacturer (Ortho Clinical Diagnostics, Buckinghamshire, UK) yielded a LoB of $7 \mathrm{ng} / \mathrm{L}$ and a LoD of $12 \mathrm{ng} / \mathrm{L}$. The package insert (version 3.0) states that the lowest concentration measurable with an imprecision (CV) less than $10 \%$ is $34 \mathrm{ng} / \mathrm{L}$. Tate and colleagues replicated this result [34], but other studies reported $10 \% \mathrm{CV}$ cutoffs of $40 \mathrm{ng} / \mathrm{L}$ [61] and $93 \mathrm{ng} / \mathrm{L}$ [35] (TABLE 1G).

The reference populations used by Tate et al and La'ulu et al have already been described above (section on the Tnl-Ultra assay) [34, 35]. Apple and colleagues recruited serum samples of 2992 healthy subjects, without additional 
specification, as well as lithium heparin plasma samples from 2000 healthy subjects, aged between 18-66 years and of which 75\% were male [36].

The $99^{\text {th }}$ percentile cutoff calculated by the manufacturer is $34 \mathrm{ng} / \mathrm{L}$, which corresponds exactly to the $10 \% \mathrm{CV}$ cutoff value. Apple et al calculated the $99^{\text {th }}$ percentile in two independent reference populations [36]. In one cohort with serum samples they replicated the cutoff value from the manufacturer, in the second reference population with heparin plasma samples a $99^{\text {th }}$ percentile concentration of $31 \mathrm{ng} / \mathrm{L}$ was reported [36]. Other studies by Tate et al, and La'ulu et al calculated much lower $99^{\text {th }}$ percentile concentrations in populations that were extensively screened [34], or comprised relatively young subjects [35]. However, the concentrations reported at an imprecision of $10 \% \mathrm{CV}$ were markedly higher than the $99^{\text {th }}$ percentile concentration in these studies.

In conclusion, various $10 \% \mathrm{CV}$ values and $99^{\text {th }}$ percentile cutoffs were observed. All studies but one [36] reported a $10 \% \mathrm{CV} / 99^{\text {th }}$ percentile ratio $>1$. Therefore, it remains controversial whether this assay can measure the $99^{\text {th }}$ percentile with a precision of $10 \%$, as quoted by the package insert.

\section{How were reference populations established? A summary}

Despite all efforts, heterogeneity in the functional sensitivity and $99^{\text {th }}$ percentile cutoffs is clearly demonstrated in TABLE 1 . The reported upper reference limits deviated from the cutoffs provided by the manufacturer, due to variations in sample size, health condition, age, gender and ethnicity. In addition, (pre-) analytical factors may have contributed to cutoff discrepancies e.g. serum versus plasma samples $[11,62]$ or the use of different platforms. Variations in the determination (e.g. using variable number of days, replicates or different platforms) of the functional sensitivity, could explain the range of $10 \%$ or $20 \%$ $\mathrm{CV}$ cutoffs that have been reported in literature.

The broad variation is apparent from the reviewed studies (66 studies; 53 publications) with sample sizes from $<100$ to $>10,000$ subjects. Most studies (40\%) included less than the advised minimum of 300 subjects and $15 \%$ of the studies collected even less than 120 subjects. Notice that with a sample size of 120,300 or 1000 subjects the $99^{\text {th }}$ percentile cutoff can be strongly affected by respectively only 1,3 or 10 subjects. 
Another concern is the variation in inclusion criteria among studies. The majority of studies $(50 \%)$ reported no inclusion criteria, suggesting that no extensive screening was performed. In $26 \%$ of the studies, the reference populations were screened by means of medical history questionnaires and/or laboratory tests. In $24 \%$ of the cases it was combined with ECG or cardiac imaging techniques such as stress echocardiography or angiography. Herein, no reference population met the selection criteria described in the guidelines [6] However, in four studies the guidelines were nearly accomplished, reporting a reference population comprised of more than 300 individuals and screened by means of non-invasive imaging [29, 42, 50,55]. It must be noted that in two of these studies the same reference population was used (SWISCH study) [29, 42] Importantly, studies that most extensively screened their reference population, reported lower $99^{\text {th }}$ percentile cutoff concentrations $[25,29,34,41,47,50]$. Evidently, in smaller reference populations more stringent screening methods can be performed, improving the composition of the reference population [25, $27,34,59]$. This is counterbalanced by the fact that a small sample size makes the $99^{\text {th }}$ percentile cutoff more prone to outliers.

Ideally, the distribution of gender and age in the reference population resembles those of a typical group of AMI patients $[6,15]$. However, age distribution in the reference populations varied widely among studies. A majority chose a broad age distribution (25-75 years), but some reference populations were composed of merely younger $[35,60]$ or older subjects $[42,50]$. Only two publications use a group of reference individuals in the same age category as AMI patients ( $>45$ years) $[42,50]$. The $99^{\text {th }}$ percentile cutoff concentration had a tendency to increase with age $[22,25,42,50,54]$. This supports recent ROC analysis from Reiter et al who reported that optimal diagnosis for AMI was achieved at higher cutoff concentrations in elderly (54 ng/L) as compared to younger subjects (17 $\mathrm{ng} / \mathrm{L})$ [63]. Moreover, gender specific differences in the $99^{\text {th }}$ percentile cutoff concentration are frequently reported. The cTnT and cTnl assays measured respectively 1.7- and 1.2-fold higher cTn levels in men than women [21, 22, 24, $25,34,49,54,55]$. Also ethnicity was reported to affect the $99^{\text {th }}$ percentile cutoff [46], although others contradict this finding [19, 44]. Taken together, these findings add to the existing body of evidence that gender and age influence cTn 
levels [64]. Future research should reveal whether gender- and age-specific cutoff values can improve diagnostic or prognostic performance.

\section{Current status on biological variation}

The NACB recommends using the rising and/or falling pattern of cardiac troponin obtained by serial measurements. They propose a $\geq 20 \%$ change in cTn concentration to be suggestive of AMI [4]. A twenty percent difference exceeds 3 analytical standard deviations, but it neglects the contribution of the biological variation to cardiac troponin levels when measuring in the lower range [65]. A better approach may be the reference change value (RCV). The RCV is established in healthy individuals and calculated as follows: $R C V=z \times \sqrt{2} \times \sqrt{C V_{A}^{2}+C V_{I}^{2}}$; where $\mathrm{z}$ is the standard deviation for a certain probability [65]. , and $\mathrm{CV}_{\mathrm{A}}$ and $C \mathrm{~V}_{\mathrm{I}}$ represent the analytical and biological variation respectively. When troponin concentrations are measured at two time points, and the subtraction of both values exceeds a critical value, defined by the RCV, the change is considered significant. Using this approach, Wu et al established the log-normal RCV for a hs-cTnl assay (Erenna Immunoassays, Singulex). They concluded that an increase of $48 \%$ or a decrease of $32 \%$ over a four hour period (short term), and $+81 \%$ or $-32 \%$ change over an eight week period (long term) is required [66]. Log-normal RCVs have also been calculated for cTnT using the hs-cTnT assay [20]. For short term changes, the cTnT concentration must rise $85 \%$ to exceed the critical difference, whereas for long term changes a $>3$ fold increase is required.

Another way to express the biological variation is the index of individuality (II). The II can be calculated by the simplified formula: $C_{1} / C V_{G}$ (within subject variation/between subject variation) [67]. By definition, reference intervals are more useful for biomarkers with a high II (>1.4) than for markers with a low II $(<0.6)$ [68]. For cTnl, the $\mathrm{CV}_{\mathrm{I}}$ is smaller than the $\mathrm{CV}_{\mathrm{G}}$, both for short-term and long-term measurements ( $9.7 \%$ versus $57 \%$, and $14 \%$ versus $63 \%$ respectively) [66]. This results in a short-term II of 0.21 and a long term II of 0.39 . When the II is low as for cTnl, it has been proposed to subdivide the reference population into more homogenous reference cohorts, which will improve the II and the usefulness of the marker in clinical practice [67]. The short- and long-term II for 
CTnT were higher, 0.84 and 1.4 , respectively $\left(\mathrm{CV}_{1}, 48 \%\right.$ and $94 \% ; \mathrm{CV}_{\mathrm{G}}, 86 \%$ and $94 \%$ for short- and long-term, respectively) [20]. Overall, CTnT seems to be more useful than cTnl, but with only one cTnl and one cTnT study published, more studies are required for a more decisive conclusion.

\section{Conclusion and future perspective}

In general, large heterogeneity in $99^{\text {th }}$ percentile cutoff values of cTn complicates the diagnosis of myocardial infarction. Major causes are lack of cardiac troponin assay harmonization, and non-standardized selection of individuals in healthy reference populations. We reviewed the literature on reference populations for seven (high-)sensitivity assays used in clinical practice and found that the upper reference limits deviated from the package inserts for all assays, although this was less apparent for the hs-cTnT assay.

As for now, the best way to establish a reference population is still debatable. From all reviewed literature, no reference populations were collected according to the guidelines $[5,6]$ although three nearly met the required standards $[29,42$, $50,55]$. A total of $40 \%$ of the studies collected less than the advised minimum of 300 subjects for reliable estimation of the $99^{\text {th }}$ percentile cutoff and $50 \%$ of the studies did not report their inclusion criteria. Moreover, there is important suggestive evidence that substantially lower cutoff values were calculated in studies that applied the most stringent selection criteria to their reference group. The available literature supports the concept that age and gender are important determinants of cardiac cTn levels. Men have on average 1.7- and 1.2-fold higher cTn levels, measured with the cTnT and cTnl assays respectively, and the $99^{\text {th }}$ percentile cutoff concentration increases with age $[22,25,42,50,54]$.

There is thus need for a large and highly screened population of cardio-healthy subjects that are preferably gender-and age-matched for AMI patients. As proposed by the IFCC, multicentre studies can be the answer to heterogeneity in reference populations: a large number of reference subjects, collected at national scale that can be adopted by clinical laboratories to determine the $99^{\text {th }}$ percentile cutoff values [69]. Alternatively, the optimal cutoff value can be defined by ROC analyses [63], reference change values, or the $97.5^{\text {th }}$ percentile can be chosen as diagnostic cutoff [1], which would be less sensitive to outliers. 
Further research should reveal whether more stringent selection criteria and gender/age-specific cutoffs can optimize the use of cTn for diagnostic and prognostic purposes, or whether alternative approaches such as the use of reference change values have added value.

TABLE 1. Analytical characteristics, $99^{\text {th }}$ percentile cutoffs and baseline characteristics of the reference study populations of seven commercially available cTn assays, classified as either guideline acceptable or clinically usable $[8]^{*}$ (next page)

* BMI, indicates body mass index; CVD, cardiovascular disease; Li, Lithium; NT-proBNP, N-terminus pro- Brain Natriuretic Peptide; n.s., not significant; -, not reported; ${ }^{a}$, Intra-assay imprecision of $5 \%$ at $10 \mathrm{ng} / \mathrm{L}$ and $1 \%$ at $100 \mathrm{ng} / \mathrm{L} ;{ }^{b}, \mathrm{CV}$ at $13.5 \mathrm{ng} / \mathrm{L}<10 \% ;^{c}$, Mean $\pm S D ;{ }^{d}$, Significant differences in $\mathrm{CTnT}$ concentrations between the same decades of age in males and females; ${ }^{e}, 10 \%$ CV cutoffs were all <99th percentile, except for one; ${ }^{f}$, Mean; ${ }^{g}$, Mean (95\% Confidence Interval); ${ }^{h}$, as quoted by the manufacturer ${ }^{i}$, median age ${ }^{j}{ }$, Intra-assay imprecision of $9.6 \%$ at $11 \mathrm{ng} / \mathrm{L}_{;}{ }^{k}, 42 \%$ Malays, 30\% Chinese, $27 \%$ Indians; ', Mean of ADVIA Centaur CP and ADVIA Centaur platform; ${ }^{m}, 20 \%$ CV cutoff concentration; ${ }^{n}$, Mean \pm 2 SD 


\begin{tabular}{|c|c|c|c|c|c|c|c|c|c|c|c|c|c|}
\hline 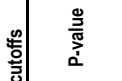 & & & & $\begin{array}{l}\overline{0} \\
\dot{\vec{j}} \\
0\end{array}$ & $\cdot$ & 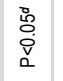 & $\begin{array}{l}\bar{\delta} \\
\text { ò } \\
\text { ñ }\end{array}$ & & $\begin{array}{l}\bar{D} \\
\dot{D} \\
0\end{array}$ & $\begin{array}{l}\bar{o} \\
\dot{0} \\
\text { on }\end{array}$ & d. & $\begin{array}{l}\bar{\delta} \\
\text { o. } \\
\dot{0} \\
\text { م. }\end{array}$ & \\
\hline 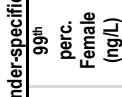 & & & & 웅 & . & ' & $\stackrel{\infty}{\infty}$ & ' & $\infty$ & $=$ & & & \\
\hline 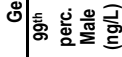 & & ' & ' & $\stackrel{\llcorner}{ \pm}$ & . & . & 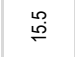 & ' & $\stackrel{\infty}{\sim}$ & $\stackrel{\leftrightarrow}{\stackrel{\sim}{\sim}}$ & ' & . & \\
\hline 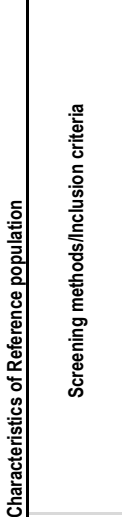 & & 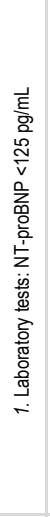 & & 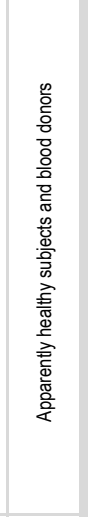 & 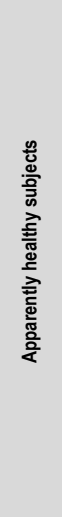 & & 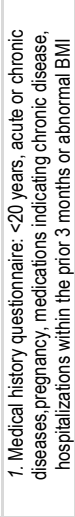 & 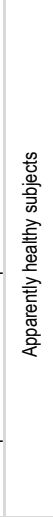 & 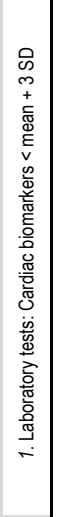 & 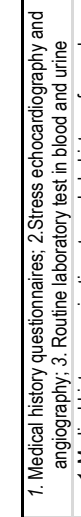 & 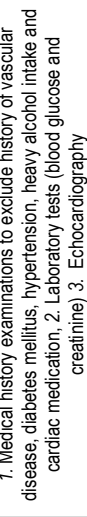 & 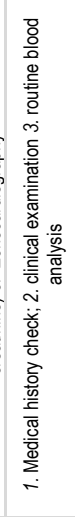 & 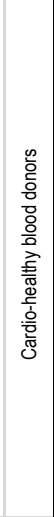 \\
\hline 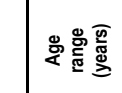 & & . & ' & 空离 & . & 蓉 & 容施 & $\frac{\text { o }}{m}$ & 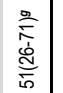 & 常 & 空 & 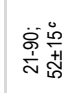 & $\begin{array}{l}\stackrel{\circ}{m} \\
\stackrel{p}{+1} \\
\stackrel{p}{0}\end{array}$ \\
\hline 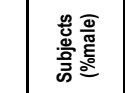 & & 辰 & $\begin{array}{l}\text { त्ञ } \\
\text { 岕 } \\
\text { }\end{array}$ & 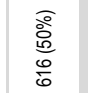 & 离 & 竞 & 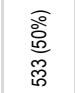 & 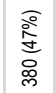 & 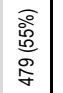 & 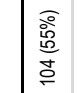 & 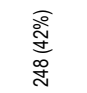 & 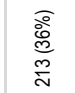 & 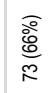 \\
\hline 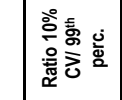 & & & $\underset{\dot{v}}{\stackrel{8}{v}}$ & $\stackrel{8}{\circ}$ & ชั & $\stackrel{\infty}{\infty}$ & $\underset{\mathrm{v}}{\stackrel{8}{\mathrm{v}}}$ & î & $\stackrel{\leftrightarrow}{\circ}$ & $\stackrel{\leftrightarrow}{\circ}$ & $\stackrel{9}{=}$ & 苦 & \\
\hline 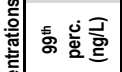 & & $\simeq$ & $\stackrel{\mathscr{\rho}}{\stackrel{p}{\rho}}$ & 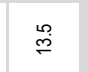 & \pm & \pm & $\stackrel{\sim}{\mathcal{I}}$ & $\stackrel{\circ}{\llcorner}$ & $\stackrel{\circ}{0}$ & $\stackrel{\leftrightarrow}{\sim}$ & 占 & $\stackrel{\circ}{\circ}$ & F \\
\hline $\begin{array}{l}\text { 형혈 } \\
\text { 홀 }\end{array}$ & & . & e & $\stackrel{m}{=}$ & $m$ & $\simeq$ & • & $\stackrel{\stackrel{\rho}{F}}{\stackrel{F}{F}}$ & o & $\stackrel{\stackrel{\rho}{E}}{\stackrel{5}{F}}$ & $\stackrel{\llcorner}{\stackrel{\infty}{\infty}}$ & స్ & - \\
\hline 오홀 & & - & $\stackrel{\circ}{\&}$ & is & is & $\sim$ & م & . & . & in & & . & $m$ \\
\hline 옿 & & & $\sim$ & m & m & . & $m$ & . & - & के & & . & \\
\hline 啇 & क्ष & & & 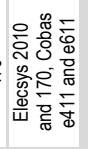 & . & 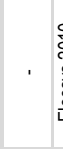 & 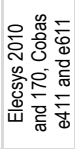 & $\begin{array}{l}8 \\
80 \\
0 \\
000 \\
0 \\
0\end{array}$ & $\begin{array}{l}\circ \\
\stackrel{0}{2} \\
\frac{w}{3} \\
\frac{\tilde{s}}{w}\end{array}$ & 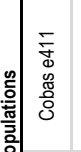 & 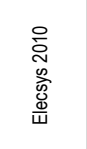 & 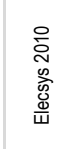 & $\begin{array}{l}\text { 음 } \\
\frac{0}{3} \\
\frac{3}{3} \\
\frac{w}{w}\end{array}$ \\
\hline 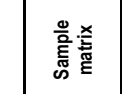 & 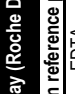 & 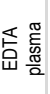 & $\varepsilon$ & & . & . & . & 駡 & 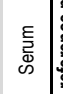 & 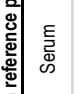 & 壹 & 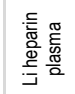 & 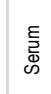 \\
\hline 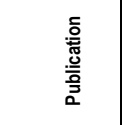 & 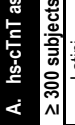 & 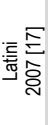 & & 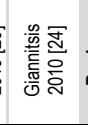 & 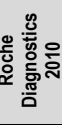 & 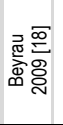 & 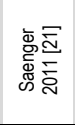 & & 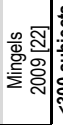 & 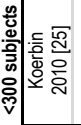 & 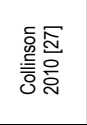 & 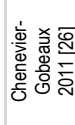 & \\
\hline
\end{tabular}




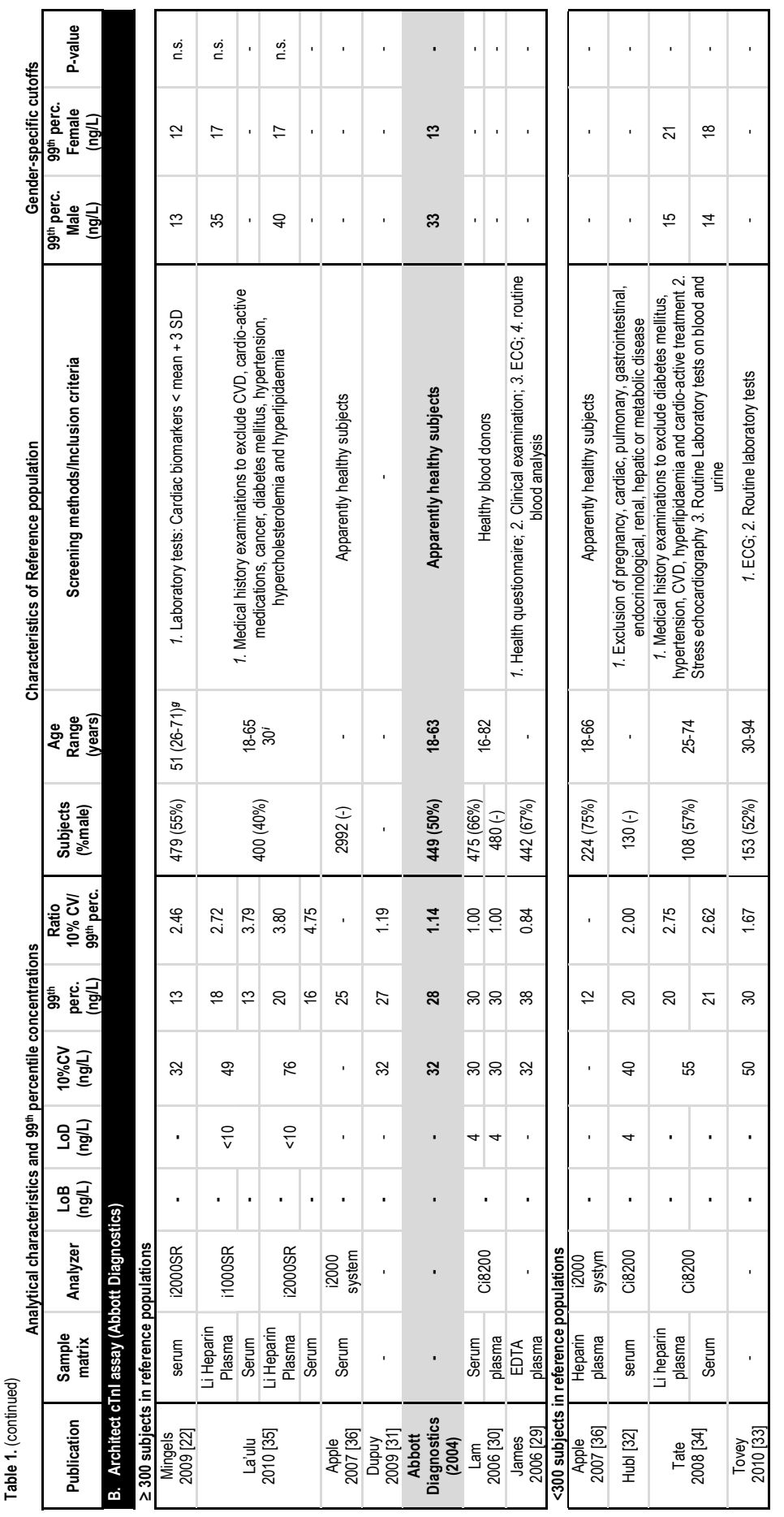




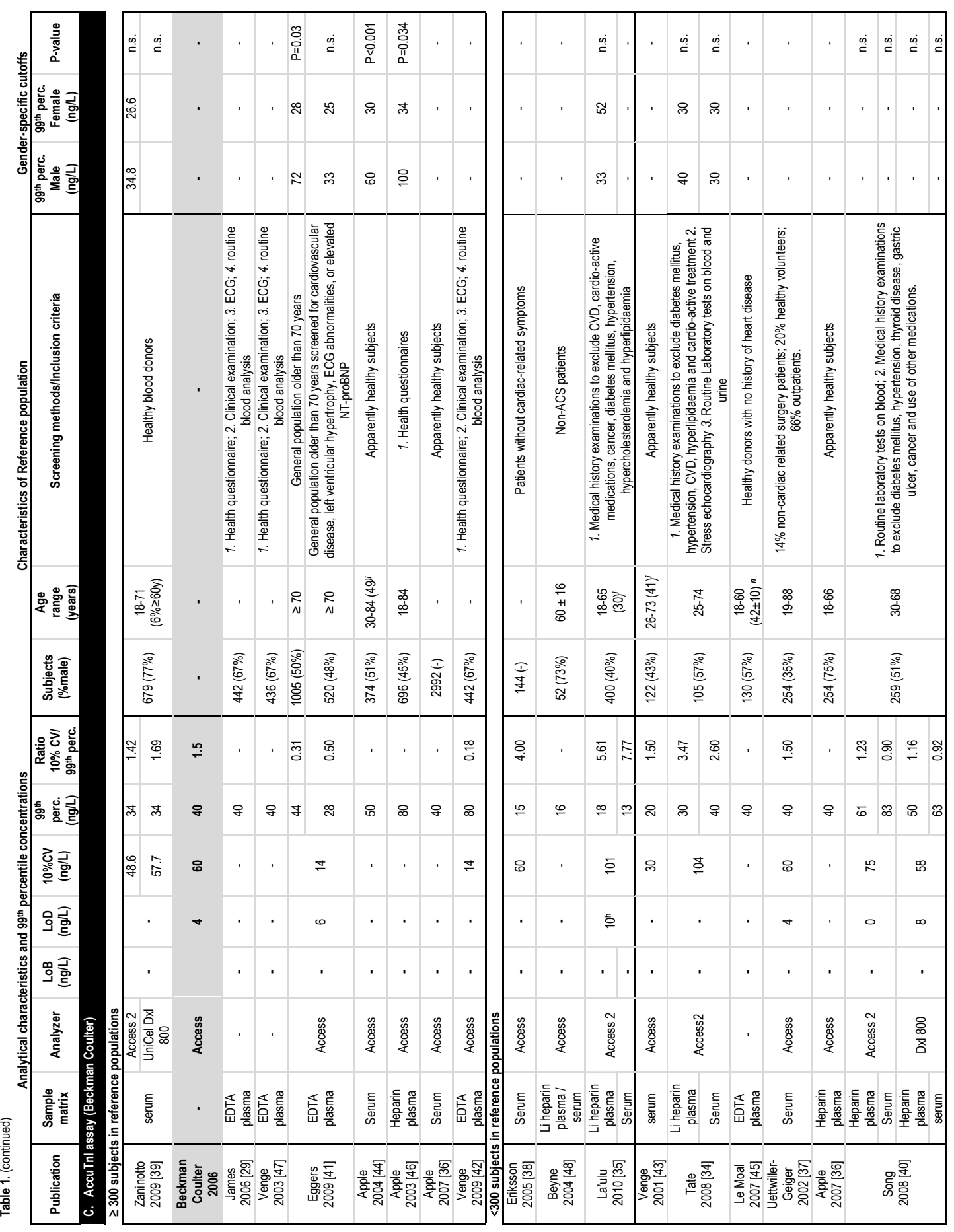




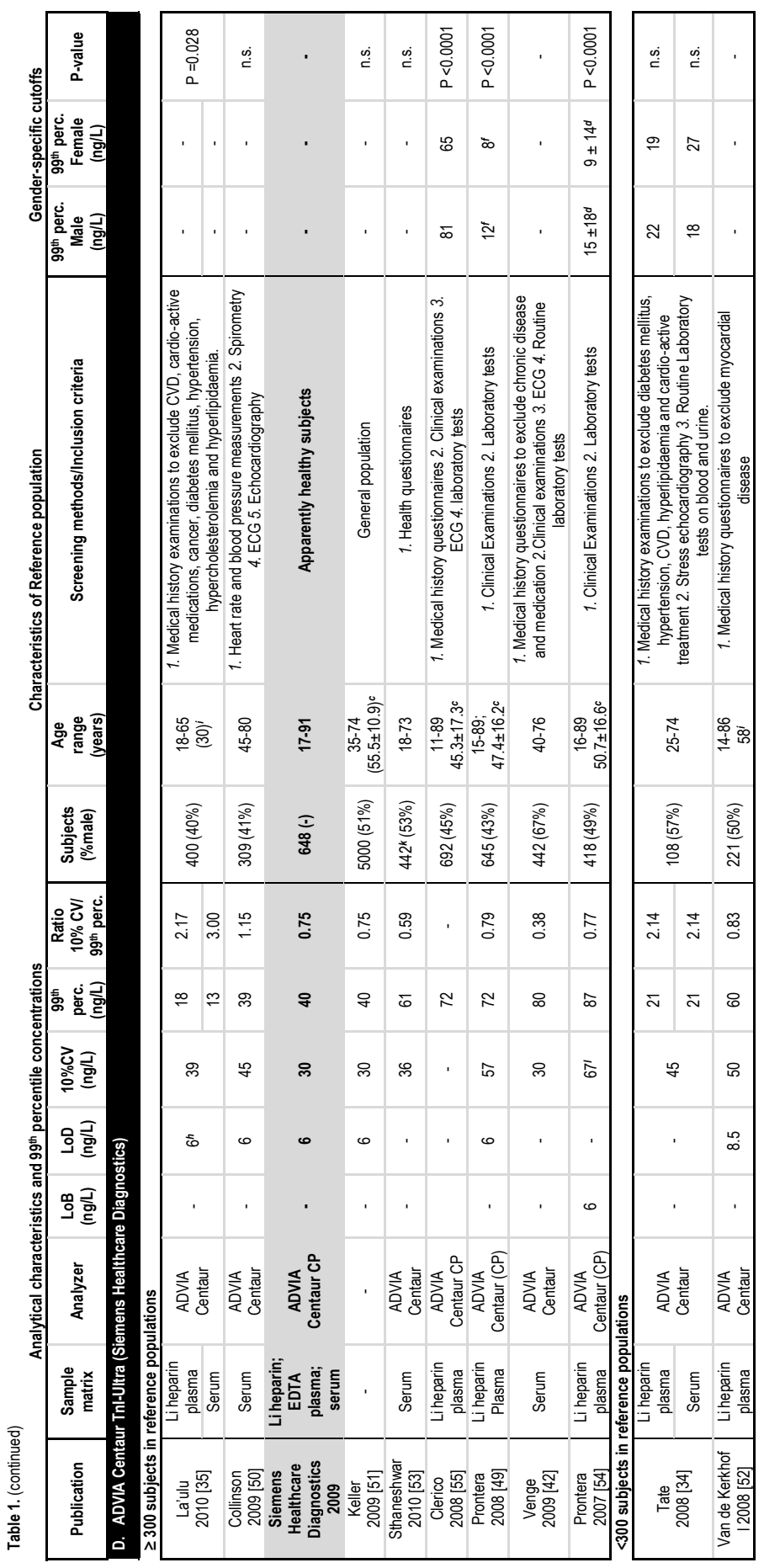




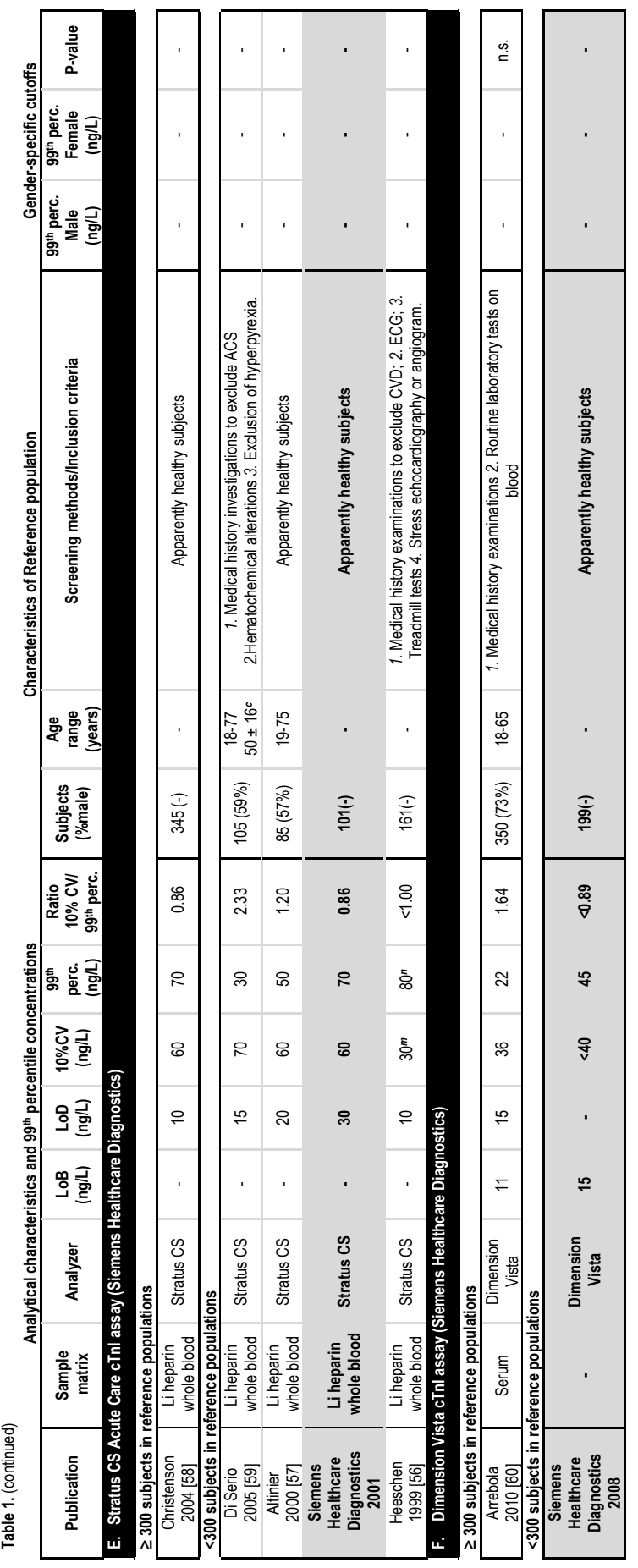




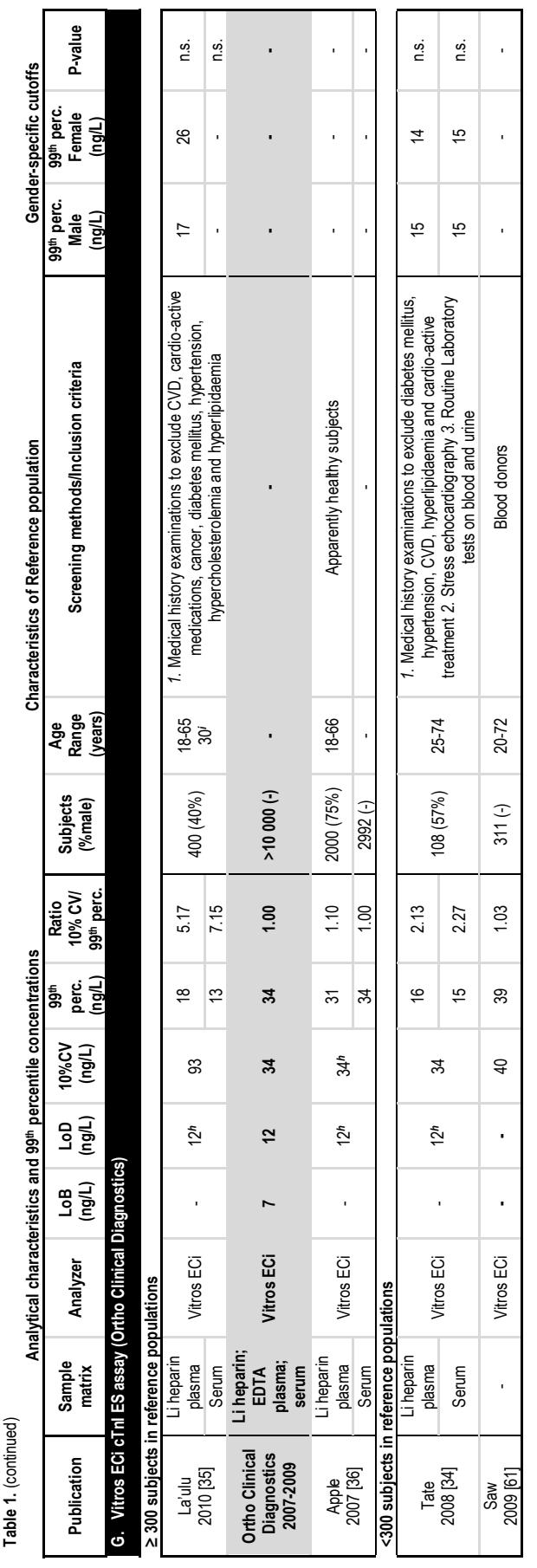




\section{References}

1. Wu, A.H., F.S. Apple, W.B. Gibler, et al., National Academy of Clinical Biochemistry Standards of Laboratory Practice: recommendations for the use of cardiac markers in coronary artery diseases. Clin Chem, 1999. 45(7): p. 1104-21.

2. Alpert, J.S., K. Thygesen, E. Antman, et al., Myocardial infarction redefined--a consensus document of The Joint European Society of Cardiology/American College of Cardiology Committee for the redefinition of myocardial infarction. J Am Coll Cardiol, 2000. 36(3): p. 959-69.

3. Thygesen, K., J.S. Alpert, H.D. White, et al., Universal definition of myocardial infarction. Circulation, 2007. 116(22): p. 2634-53.

4. Morrow, D.A., C.P. Cannon, R.L. Jesse, et al., National Academy of Clinical Biochemistry Laboratory Medicine Practice Guidelines: Clinical characteristics and utilization of biochemical markers in acute coronary syndromes. Circulation, 2007. 115(13): p. 356-75.

5. Apple, F.S., R.L. Jesse, L.K. Newby, et al., National Academy of Clinical Biochemistry and IFCC Committee for Standardization of Markers of Cardiac Damage Laboratory Medicine Practice Guidelines: analytical issues for biochemical markers of acute coronary syndromes. Clin Chem, 2007. 53(4): p. 547-51.

6. Thygesen, K., J. Mair, H. Katus, et al., Recommendations for the use of cardiac troponin measurement in acute cardiac care. Eur Heart J, 2010. 31(18): p. 2197-204.

7. International Federation of Clinical Chemistry and Laboratory Medicine (IFCC) Working group on the Standardization of Troponin I (WG-TNI). Troponin assay analytical characteristics. [cited 2012 June ]; Available from: http://www.ifcc.org/PDF/ScientificActivities/IFCC_Troponin_Table_vDec_2010_FINAL_ng_L _28Jan11.pdf.

8. Apple, F.S., A new season for cardiac troponin assays: it's time to keep a scorecard. Clin Chem, 2009. 55(7): p. 1303-6.

9. Jaffe, A.S., F.S. Apple, D.A. Morrow, et al., Being rational about (im)precision: a statement from the Biochemistry Subcommittee of the Joint European Society of Cardiology/American College of Cardiology Foundation/American Heart Association/World Heart Federation Task Force for the definition of myocardial infarction. Clin Chem, 2010. 56(6): p. 941-3.

10. Morrow, D.A. and E.M. Antman, Evaluation of high-sensitivity assays for cardiac troponin. Clin Chem, 2009. 55(1): p. 5-8.

11. Panteghini, M., A critical appraisal of experimental factors influencing the definition of the 99th percentile limit for cardiac troponins. Clin Chem Lab Med, 2009. 47(10): p. 1179-82.

12. Panteghini, M., Assay-related issues in the measurement of cardiac troponins. Clin Chim Acta, 2009. 402(1-2): p. 88-93.

13. de Lemos, J.A., M.H. Drazner, T. Omland, et al., Association of troponin T detected with a highly sensitive assay and cardiac structure and mortality risk in the general population. JAMA, 2010. 304(22): p. 2503-12.

14. defilippi, C.R., J.A. de Lemos, R.H. Christenson, et al., Association of serial measures of cardiac troponin $T$ using a sensitive assay with incident heart failure and cardiovascular mortality in older adults. JAMA, 2010. 304(22): p. 2494-502.

15. Solberg, H.E., Establishment and Use of Reference Values, in Tietz textbook of clinical chemistry and molecular diagnostics, C.A. Burtis, E.R. Ashwood, and D.E. Bruns, Editors. 2006, Elsevier Saunders. p. 425-48.

16. Pulkki, K., J. Suvisaari, P. Collinson, et al., A pilot survey of the use and implementation of cardiac markers in acute coronary syndrome and heart failure across Europe. The CARdiac MArker Guideline Uptake in Europe (CARMAGUE) study. Clin Chem Lab Med, 2009. 47(2): p. 227-34.

17. Latini, R., S. Masson, I.S. Anand, et al., Prognostic value of very low plasma concentrations of troponin $T$ in patients with stable chronic heart failure. Circulation, 2007. 116(11): p. 1242-9. 
18. Beyrau, R., S. Braun, A. Dolci, et al., Multicentre evaluation of a high sensitive Elecsys troponin T assay [Abstract]. Clin Chem, 2009. 55(6): p. A70.

19. Vasatova, M., R. Pudil, M. Tichy, et al., High-sensitivity troponin T as a marker of myocardial injury after radiofrequency catheter ablation. Ann Clin Biochem, 2010: p. 1-3.

20. Vasile, V.C., A.K. Saenger, J.M. Kroning, et al., Biological and analytical variability of a novel high-sensitivity cardiac troponin T assay. Clin Chem, 2010. 56(7): p. 1086-90.

21. Saenger, A.K., R. Beyrau, S. Braun, et al., Multicenter analytical evaluation of a highsensitivity troponin T assay. Clin Chim Acta, 2011. 412(9-10): p. 748-54.

22. Mingels, A., L. Jacobs, E. Michielsen, et al., Reference population and marathon runner sera assessed by highly sensitive cardiac troponin $\mathrm{T}$ and commercial cardiac troponin $\mathrm{T}$ and $\mathrm{I}$ assays. Clin Chem, 2009. 55(1): p. 101-8.

23. Aw, T.C., S.K. Pua, L. Yew, et al., Performance of a new rapid automated high-sensitive troponin T (hsTnT) immunoassay [Abstract]. Clinical chemistry, 2010. 56(6): p. C88.

24. Giannitsis, E., K. Kurz, K. Hallermayer, et al., Analytical validation of a high-sensitivity cardiac troponin T assay. Clin Chem, 2010. 56(2): p. 254-61.

25. Koerbin, G., J.R. Tate, and P.E. Hickman, Analytical characteristics of the Roche highly sensitive troponin $\mathrm{T}$ assay and its application to a cardio-healthy population. Ann Clin Biochem, 2010: p. 1-5.

26. Chenevier-Gobeaux, C., C. Meune, M.C. Blanc, et al., Analytical evaluation of a highsensitivity troponin T assay and its clinical assessment in acute coronary syndrome. Ann Clin Biochem, 2011.

27. Collinson, P., Y. Heung, D. Gaze, et al., The assay performance and 99th percentile reference limit for cardiac troponin $T$ using a new high sensitivity assay in a fully characterised reference population [Abstract]. Clinical Chemistry, 2010. 56(6): p. C50.

28. Wallace, T.W., S.M. Abdullah, M.H. Drazner, et al., Prevalence and determinants of troponin T elevation in the general population. Circulation, 2006. 113(16): p. 1958-65.

29. James, S., M. Flodin, N. Johnston, et al., The Antibody Configurations of Cardiac Troponin I Assays May Determine Their Clinical Performance. Clin Chem, 2006. 52(5): p. 832-7.

30. Lam, Q., M. Black, O. Youdell, et al., Performance evaluation and subsequent clinical experience with the Abbott Automated Architect STAT Troponin-I assay. Clin Chem, 2006. 52(2): p. 298-300.

31. Dupuy, A.M., S. Bouvier, A.S. Bargnoux, et al., Clinical efficacy of two cardiac troponin I assays. Clin Chem Lab Med, 2009. 47(8): p. 1013-5.

32. Hubl, W., T. Demant, and E. Gladrow, Evaluation of the ARCHITECT STAT troponin-I assay. Clin Lab, 2005. 51(5-6): p. 251-5.

33. Tovey, J.A., A. Brain, and M.D. Penney, Calculation of the 99th percentile upper reference limit and clinical decision concentration of the Abbott STAT Tnl assay. Ann Clin Biochem, 2010. 47(Pt 6): p. 576-7.

34. Tate, J.R., W. Ferguson, R. Bais, et al., The determination of the 99th centile level for troponin assays in an Australian reference population. Ann Clin Biochem, 2008. 45(Pt 3): p. 275-88.

35. La'ulu, S.L. and W.L. Roberts, Performance characteristics of five cardiac Troponin I assays. Clin Chim Acta, 2010. 411(15-16): p. 1095-101.

36. Apple, F.S. and M.M. Murakami, Serum and plasma cardiac troponin I 99th percentile reference values for 3 2nd-generation assays. Clin Chem, 2007. 53(8): p. 1558-60.

37. Uettwiller-Geiger, D., A.H. Wu, F.S. Apple, et al., Multicenter evaluation of an automated assay for troponin I. Clin Chem, 2002. 48(6 Pt 1): p. 869-76.

38. Eriksson, S., T. Ilva, C. Becker, et al., Comparison of cardiac troponin I immunoassays variably affected by circulating autoantibodies. Clin Chem, 2005. 51(5): p. 848-55.

39. Zaninotto, M., M.M. Mion, E. Novello, et al., Precision performance at low levels and 99th percentile concentration of the Access AccuTnl assay on two different platforms. Clin Chem Lab Med, 2009. 47(3): p. 367-71. 
40. Song, S., B. Suh, S.G. Lee, et al., Comparison of $10 \%$ coefficient of variation and 99th percentile cutoff of troponin I in serum and heparinized plasma using Access 2 and DxI 800 . Clin Chim Acta, 2009. 399(1-2): p. 117-8.

41. Eggers, K.M., A.S. Jaffe, L. Lind, et al., Value of cardiac troponin I cutoff concentrations below the 99th percentile for clinical decision-making. Clin Chem, 2009. 55(1): p. 85-92.

42. Venge, P., S. James, L. Jansson, et al., Clinical performance of two highly sensitive cardiac troponin I assays. Clin Chem, 2009. 55(1): p. 109-16.

43. Venge, P., B. Lindahl, and L. Wallentin, New generation cardiac troponin I assay for the access immunoassay system. Clin Chem, 2001. 47(5): p. 959-61.

44. Apple, F.S. and M.M. Murakami, Serum 99th percentile reference cutoffs for seven cardiac troponin assays. Clin Chem, 2004. 50(8): p. 1477-9.

45. Le Moal, E., I. Giuliani, J.P. Bertinchant, et al., Earlier detection of myocardial infarction by an improved cardiac Tnl assay. Clin Biochem, 2007.

46. Apple, F.S., H.E. Quist, P.J. Doyle, et al., Plasma 99th Percentile Reference Limits for Cardiac Troponin and Creatine Kinase MB Mass for Use with European Society of Cardiology/American College of Cardiology Consensus Recommendations. Clin Chem, 2003. 49(8): p. 1331-6.

47. Venge, P., N. Johnston, B. Lagerqvist, et al., Clinical and analytical performance of the liaison cardiac troponin I assay in unstable coronary artery disease, and the impact of age on the definition of reference limits. A FRISC-II substudy. Clin Chem, 2003. 49(6 Pt 1): p. 880-6.

48. Beyne, P., E. Bouvier, P. Werner, et al., Emergency department triage of patients with acute chest pain: definition of cardiac troponin I decisional value to manage patients without electrocardiographic evidence of ischemia. Clin Chem Lab Med, 2004. 42(5): p. 556-9.

49. Prontera, C., A. Fortunato, S. Storti, et al., Evaluation of analytical performance of Advia ${ }^{\circledR}$ Tnl ultra immunoassay and comparison with Access ${ }^{\circledR}$ AccuTnI(TM) method. Immunoanalyse \& Biologie Spécialisée, 2008. 23(5): p. 311-318.

50. Collinson, P.O., O. Clifford-Mobley, D. Gaze, et al., Assay imprecision and 99th-percentile reference value of a high-sensitivity cardiac troponin I assay. Clin Chem, 2009. 55(7): p. 1433-4.

51. Keller, T., T. Zeller, D. Peetz, et al., Sensitive troponin I assay in early diagnosis of acute myocardial infarction. N Engl J Med, 2009. 361(9): p. 868-77.

52. van de Kerkhof, D., B. Peters, and V. Scharnhorst, Performance of the Advia Centaur second-generation troponin assay Tnl-Ultra compared with the first-generation cTnl assay. Ann Clin Biochem, 2008. 45(Pt 3): p. 316-7.

53. Sthaneshwar, P., F.A. Jamaluddin, and Y.S. Fan, Reference value for cardiac troponin I in a multi-ethnic group. Pathology, 2010. 42(5): p. 454-6.

54. Prontera, C., A. Fortunato, S. Storti, et al., Evaluation of analytical performance of the Siemens ADVIA Tnl ultra immunoassay. Clin Chem, 2007. 53(9): p. 1722-3.

55. Clerico, A., A. Fortunato, A. Ripoli, et al., Distribution of plasma cardiac troponin I values in healthy subjects: pathophysiological considerations. Clin Chem Lab Med, 2008. 46(6): p. 804-8.

56. Heeschen, C., B.U. Goldmann, L. Langenbrink, et al., Evaluation of a rapid whole blood ELISA for quantification of troponin I in patients with acute chest pain. Clin Chem, 1999. 45(10): p. 1789-96.

57. Altinier, S., M. Mion, A. Cappelletti, et al., Rapid measurement of cardiac markers on Stratus CS. Clin Chem, 2000. 46(7): p. 991-3.

58. Christenson, R.H., D.R. Cervelli, R.S. Bauer, et al., Stratus CS cardiac troponin I method: performance characteristics including imprecision at low concentrations. Clin Biochem, 2004. 37(8): p. 679-83.

59. Di Serio, F., G. Amodio, L. Varraso, et al., Integration between point-of-care cardiac markers in an emergency/cardiology department and the central laboratory: methodological and preliminary clinical evaluation. Clin Chem Lab Med, 2005. 43(2): p. 202-9.

60. Arrebola, M.M., J.A. Lillo, M.J. Diez De Los Rios, et al., Analytical performance of a sensitive assay for cardiac troponin I with loci technology. Clin Biochem, 2010. 43(12): p. 998-1002. 
61. Saw, S., C. Wang, and S. Sethi, Meeting the challenge of the ESC/ACC definition of myocardial infarction of the new troponin I ES on the VITROS ${ }^{\circledR} 5600$ integrated system and VITROS $^{\circledR}$ ECi immunodiagnostic system [Abstract]. Clin Chem, 2009. 55(6): p. B51.

62. van de Kerkhof, D., B. Peters, and V. Scharnhorst, Troponin I concentrations in heparinized plasma and serum differ when measured with the Advia Centaur Tnl-Ultra assay. Scand J Clin Lab Invest, 2008: p. 1-3.

63. Reiter, M., R. Twerenbold, T. Reichlin, et al., Early diagnosis of acute myocardial infarction in the elderly using more sensitive cardiac troponin assays. Eur Heart J, 2011. 32(11): p. 13791388.

64. Roger, V.L., A.S. Go, D.M. Lloyd-Jones, et al., Heart disease and stroke statistics--2011 update: a report from the American Heart Association. Circulation, 2011. 123(4): p. e18e209.

65. Fraser, C.G. and E.K. Harris, Generation and application of data on biological variation in clinical chemistry. Crit Rev Clin Lab Sci, 1989. 27(5): p. 409-37.

66. Wu, A.H., Q.A. Lu, J. Todd, et al., Short- and long-term biological variation in cardiac troponin I measured with a high-sensitivity assay: implications for clinical practice. Clin Chem, 2009. 55(1): p. 52-8.

67. Petersen, P.H., C.G. Fraser, S. Sandberg, et al., The index of individuality is often a misinterpreted quantity characteristic. Clin Chem Lab Med, 1999. 37(6): p. 655-61.

68. Harris, E.K., Effects of intra- and interindividual variation on the appropriate use of normal ranges. Clin Chem, 1974. 20(12): p. 1535-42.

69. Ceriotti, F., Common reference intervals: the IFCC position. Clin Biochem, 2009. 42(4-5): p. 297. 



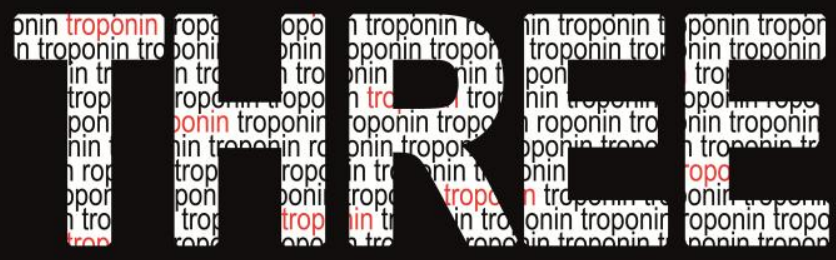

\section{HIGH-SENSITIVITY CARDIAC TROPONIN CONCENTRATIONS}

IN PATIENTS WITH CHEST DISCOMFORT:

IS IT THE HEART OR THE KIDNEYS AS WELL?

Cardinaels E, Altintas S, Versteylen M, Joosen I, Jellema L, Das M, Wildberger J, Crijns $\mathrm{H}$, Bekers $\mathrm{O}$, van Dieijen-Visser M, Kietselaer B* and Mingels $A^{*}$

*, both authors contributed equally 


\section{Abstract \\ Background}

The extent of renal influence on hs-cTn concentrations is still unknown in patients suspected for coronary artery disease (CAD) and is necessary for appropriate interpretation of low-level hs-cTn concentrations. We therefore simultaneously investigated the renal and cardiac contribution to hs-cTn concentrations in chest discomfort patients from the cardiology outpatient department.

\section{Methods}

A cross-sectional analysis was performed in 1864 patients with symptoms of chest discomfort who underwent cardiac computed tomographic angiography (CCTA). Serum samples were analyzed using hs-cTnT and hs-cTnl assays. Renal function was measured by the estimated glomerular filtration rate (eGFR), established from serum creatinine and cystatin $\mathrm{C}$. On follow-up, the incidence of cardiovascular events was assessed.

\section{Results}

Median hs-cTnT and hs-cTnl concentrations were 7.2(5.8-9.2)ng/L and (2.6(1.84.1) $\mathrm{ng} / \mathrm{L}$, respectively. Both hs-cTn measurements were more strongly associated with eGFR (hs-cTnT:st $\beta$ :-0.289; hs-cTnl:st $\beta$ :-0.222) than with cardiac imaging parameters, such as coronary calcium score (hscTnT:st $\beta: 0.078$; hs-cTnl:st $\beta: 0.092$ ), CCTA plaque severity score (hscTnT:st $\beta: 0.074 ;$ hs-cTnl:st $\beta: 0.072$ ) and left ventricular mass (hs-cTnT:st $\beta: 0.139$ ;hs-cTnl:st $\beta: 0.234$ ) (all $p \leq 0.01$ and after adjustment for traditional risk factors). Moreover, the association between eGFR and hs-cTn remained equally strong among patients with no (hs-cTnT:stßeGFR:-0.295; hs-cTnl:stßeGFR:-0.228), mild (hs-cTnT:stßeGFR:-0.290; hs-cTnl:stßeGFR:-0.176) and moderate-tosevere CAD (hs-cTnT:stßeGFR:-0.293; hs-cTnl:stßeGFR:-0.249) (all p<0.001). Both hs-cTnT and hs-cTnl remain independent prognostic markers for cardiovascular events, irrespective of renal function and CAD.

\section{Conclusion}

In patients with chest discomfort, we identified decreases in renal function and the extent of CAD as two separate contributors of troponin accumulation. Awareness of these renal influences is therefore warranted when interpreting hs-cTn concentrations. 


\section{Introduction}

Identifying chest pain patients at risk for cardiovascular events remains an ongoing challenge [1]. A promising and cost-effective way to identify those "vulnerable" patients is the use of cardiac troponins [2, 3]. Because of their unique cardiospecificity, cardiac troponins are considered the preferred biochemical markers to detect myocardial injury and to diagnose acute myocardial infarction (AMI) in particular [4]. Since the introduction of highsensitivity cardiac troponin (hs-cTn) assays, more accurate detection of low levels of circulating cardiac troponins became feasible [5]. The use of hs-cTn concentrations significantly improved the diagnostic performance in patients with acute cardiac risk[6]. Moreover, hs-cTn concentrations turned out to have an important prognostic value for acute cardiovascular events, even below the diagnostic cut-off and beyond traditional cardiovascular risk factors [7-9].

Unfortunately, the shift to more sensitive assays is accompanied by a reduction in specificity, as circulating hs-cTn levels are elevated in many other conditions besides $A M I[7,10]$. Renal dysfunction is one of those conditions in which elevated cardiac troponin concentrations are commonly detected [11, 12]. Recently it was shown in chronic kidney disease (CKD) patients that elevated hs-cTn concentrations are indeed associated with reduced renal function [13]. Therefore, the interpretation of baseline hs-cTn values in the individual patient is complicated not only by cardiac disease [14-16] but also renal dysfunction. As of yet it is unknown to what extent renal function contributes to higher cardiac troponin concentrations in patients with chest discomfort, in whom circulating hs-cTnT concentrations are mainly attributed to the presence and severity of atherosclerotic plaques [2, 3] or echocardiographic abnormalities [17-19]. Indepth understanding of the relative contribution of renal function, in addition to cardiac parameters, is of utmost importance for the use of hs-cTn values in clinical practice.

In this study, we therefore evaluate the association of serum hs-cTnT and hscTnl concentrations with renal function (as assessed by the estimated glomerular filtration rate (eGFR)), cardiac imaging measures (cardiac computed tomographic angiography (CCTA) and echocardiography), and the incidence of cardiovascular events in patients who visited the cardiology outpatient 
department with symptoms of chest discomfort. Particularly, the renal and cardiac contribution to hs-cTnT and hs-cTnl concentrations will be examined simultaneously.

\section{Materials and methods}

\section{Study cohort}

We analyzed a cohort of 1864 consecutive patients who were enrolled in the Maastricht Biomarker CT Study (ClinicalTrials.gov, NCT01671930). This cohort is comprised of patients presenting with (a)typical chest pain with a low-tointermediate pretest probability who were referred for CCTA from the cardiology outpatient department for the evaluation of coronary artery disease (CAD), in accordance with the current guidelines[1]. Included were patients of whom serum was collected. Excluded were patients with previous history ACS and patients on dialysis (due to application of contrast fluids). Additional specifications of this population have previously been published [2, 20]. This study was conducted according to the principles of the Declaration of Helsinki and approved by the local ethics committee. Written informed consent was obtained from all patients.

\section{Biochemical analysis}

Serum samples were collected immediately before CCTA, processed within 2 hours and directly stored at $-80{ }^{\circ} \mathrm{C}$ until analysis. Total cholesterol, triglycerides, high-density and low-density lipoprotein concentrations were measured as previously described [2]. Serum creatinine, cystatin C and cTnT concentrations were measured on the Cobas 6000 analyzer (Roche Diagnostics) in a fresh aliquot. Creatinine concentrations were assessed using the enzymatic method (Roche). Cystatin $\mathrm{C}$ was measured using a new particle-enhanced turbidimetric assay (Gentian AS), that was standardized against the certified ERMDA471/IFCC cystatin C reference material [21]. The glomerular filtration rate was estimated using the Chronic Kidney Disease Epidemiology Collaboration equations[22] using serum creatinine and cystatin $C$ concentrations. CTnT concentrations were determined using the high-sensitivity cTnT assay (Roche; lotnumber 167650 ), with a $99^{\text {th }}$ percentile upper reference limit of $14 \mathrm{ng} / \mathrm{L}$ and a 
$10 \%$ coefficient of variation cut-off at $13 \mathrm{ng} / \mathrm{L}$. Gender-specific cutoffs were reported at $14.5 \mathrm{ng} / \mathrm{L}$ and $10 \mathrm{ng} / \mathrm{L}$ for males and females, respectively [5]. cTnl measurements were performed on the ARCHITECT i2000SR platform using the precommercial ARCHITECT STAT high-sensitivity troponin I (hs-cTnl) assay (Abbott Laboratories). According to the manufacturer, a $10 \% \mathrm{CV}$ was reached at $4.7 \mathrm{ng} / \mathrm{L}$ and the $99^{\text {th }}$ percentile cut-off concentration at $26.2 \mathrm{ng} / \mathrm{L}$ for the overall population. Gender-specific cut-offs at $34.2 \mathrm{ng} / \mathrm{L}$ and $15.6 \mathrm{ng} / \mathrm{L}$ were also defined for males and females, respectively.

\section{Cardiac computed tomographic angiography}

CCTAs were performed from December 2007 through December 2012 and analyzed as previously described [20]. In brief, CCTA were analyzed by two experts who were blinded from hs-cTn results. The coronary calcium score (CCS) was quantified by the Agatston method[23] and luminal plaque severity as assessed by CCTA was scored as no, mild ( $<50 \%$ stenosis), moderate (50$70 \%$ stenosis) and severe ( $>70 \%$ stenosis) CAD.

\section{Echocardiography}

Echocardiography was performed in a subset of 549 patients (31\%) within a period of 3 months from the CCTA scan by an expert echocardiographist, who was blinded for hs-cTn concentrations. Transthoracic images of the left ventricle (LV) were acquired to assess morphology, function and mass (Philips IE 33, Philips Medical Systems). LV function and -mass were calculated by off-line image analysis using Xcelera software package (Philips Medical Systems), according to current ESC/AHA guidelines [24]. Left ventricular hypertrophy was defined as an LVmass $>95 \mathrm{~g} / \mathrm{m}^{2}$ in females or LVmass $>115 \mathrm{~g} / \mathrm{m}^{2}$ in males [25].

\section{Cardiovascular events}

Electronic patient records were monitored for the occurrence of cardiovascular events by two reviewers. Survival time was defined as the period from date of CCTA to date of the first cardiovascular event or June 2013. The composite study endpoints were defined in advance as major adverse cardiovascular events, encompassing cardiovascular mortality, acute coronary syndromes including $\mathrm{AMI}$ and unstable angina requiring hospitalization; and late coronary 
revascularization (>90 days after CCTA), such as percutaneous coronary interventions and coronary artery bypass grafting [26]. No records could be retrieved of 175 subjects, therefore 1689 patients (91\%) of the total cohort were included for follow-up analysis.

\section{Statistical analysis}

Comparisons of baseline characteristics were performed using the T-test for continuous variables with a normal distribution, Mann-Whitney U-test for nonnormal distributed continuous variables and Chi square test for categorical variables. Data are presented as proportions, means \pm standard deviations, and data with a non-normal distribution are given as the median (interquartile range, IQR). Pearson $R$ correlation factors were calculated with the natural logarithm (Ln) of hs-cTnT and hs-cTnl to normalize their skewed distribution.

To assess the independent association of renal and cardiovascular parameters with hs-cTn concentrations, linear regression analyses were performed with either $\operatorname{Ln}(\mathrm{hs}-\mathrm{cTnT})$ or $\mathrm{Ln}(\mathrm{hs}-\mathrm{cTnl})$ as the dependent variable. Only the cardiovascular risk factors that were significantly associated to higher hs-cTnT quartiles (TABLE 1) were entered into the multivariable model. $R^{2}$ was calculated to measure the performance of the model, and the $\mathrm{R}^{2}$ change to address the additive effect of eGFR to the model. Univariable and multivariable cox-proportional hazards models were performed to investigate the association with cardiovascular events. The discriminative ability of each predictor was assessed using the receiver-operating characteristic (ROC) curves.

Statistical analysis was performed with SPSS 20.0 (SPSS). Two sided p-values of $<0.05$ were considered statistically significant.

\section{Results}

\section{Distribution and determinants of hs-cTnT and hs-cTnl}

Median (IQR) hs-cTn concentrations in this cohort were 7.2 (5.8-9.2) ng/L for hs-cTnT and $2.6(1.8-4.1) \mathrm{ng} / \mathrm{L}$ for hs-cTnl. Of all patients, 6.6\% $(n=123)$ were above the cutoff of hs-cTnT (14 ng/L) and $2.1 \%(n=30)$ above the cutoff of hscTnl (26.2 ng/L). The majority of all patients $(72 \%)$ had a normal renal function $\left(\right.$ eGFR $>90 \mathrm{~mL} / \mathrm{min} / 1.73 \mathrm{~m}^{2}$ ) and $98 \%$ had an eGFR above $60 \mathrm{~mL} / \mathrm{min} / 1.73 \mathrm{~m}^{2}$. 
The baseline characteristics are presented in TABLE 1 and illustrate that increasing quartiles of hs-cTnT and hs-cTnl were highly associated with traditional cardiovascular risk factors such as advancing age, male sex and hypertension. The association between $\mathrm{Ln}(\mathrm{hs}-\mathrm{cTnT})$ and $\mathrm{Ln}(\mathrm{hs}-\mathrm{cTnl})$ was only moderate (Pearson R: 0.635; $p<0.001$ and cohen $\mathrm{k}$ of $0.27 ; \mathrm{p}<0.001$; SUPPLEMENTAL TABLE 1).

Independent association of cardiovascular disease and renal function with hs-cTn concentrations

Univariable regression analysis demonstrated that hs-cTn concentrations are significantly associated with eGFR (hs-cTnT: R:-0.396; hs-cTnl: R:-0.251; SUPPLEMENTAL FIGURE 1), regardless of the algorithm that is used to estimate GFR (SUPPLEMENTAL FIGURE 2). Also, both hs-cTnT and hs-cTnl concentrations are significantly correlated with CCS (hs-cTnT: R:0.279; hs-cTnl: $\mathrm{R}: 0.213$ ) and CCTA plaque severity (hs-cTnT: R:0.307; hs-cTnl, R:0.230) (TABLE 2 and SUPPLEMENTAL FIGURE 3, ALL P<0.001).

As displayed by FIGURE 1, when adding renal and CT parameters as explanatory variables for hs-cTn concentrations in a multivariable regression model, both eGFR, CCS as CCTA plaque severity were identified as independent predictors (TABLE 2, unadjusted multivariable model). 


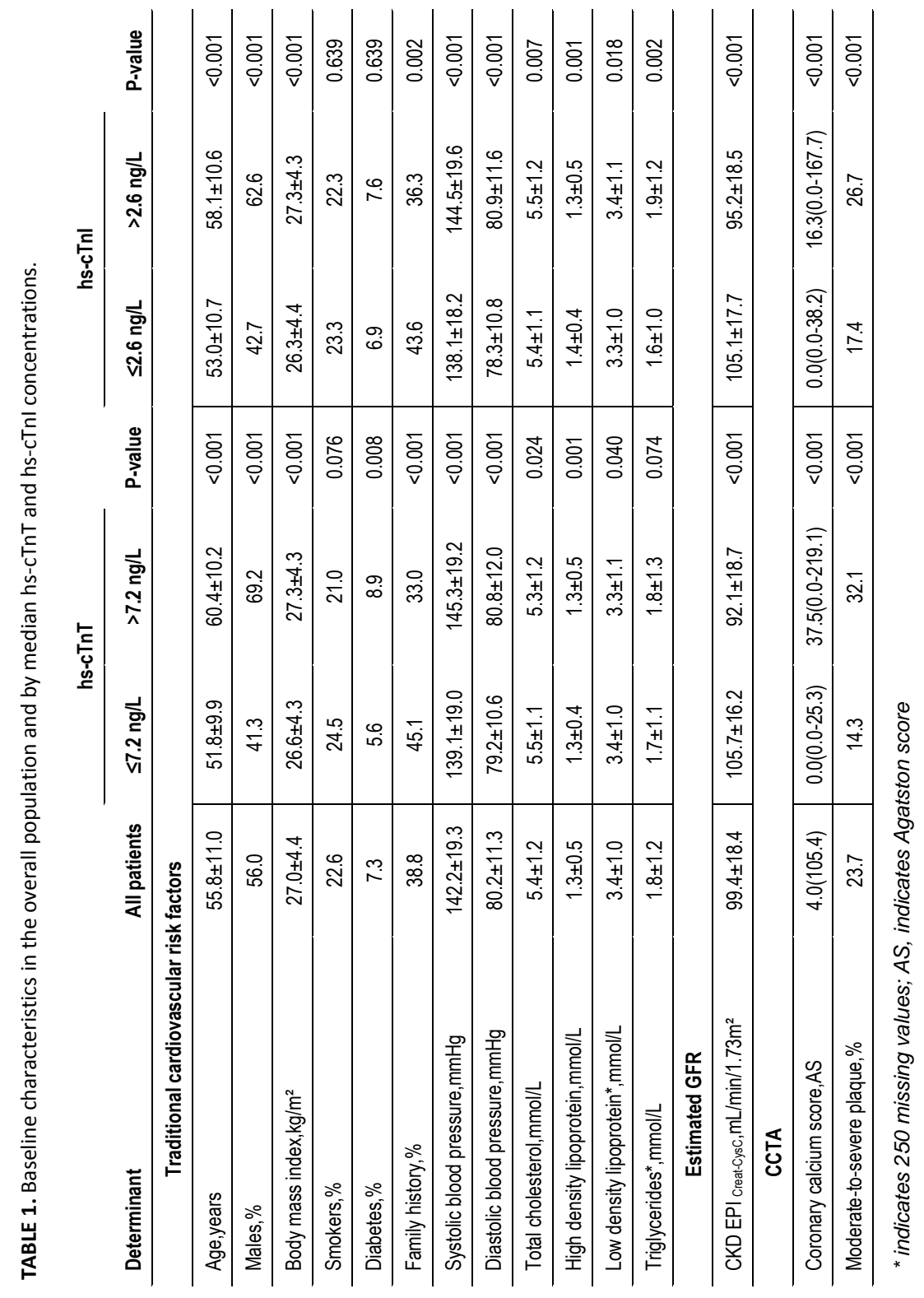



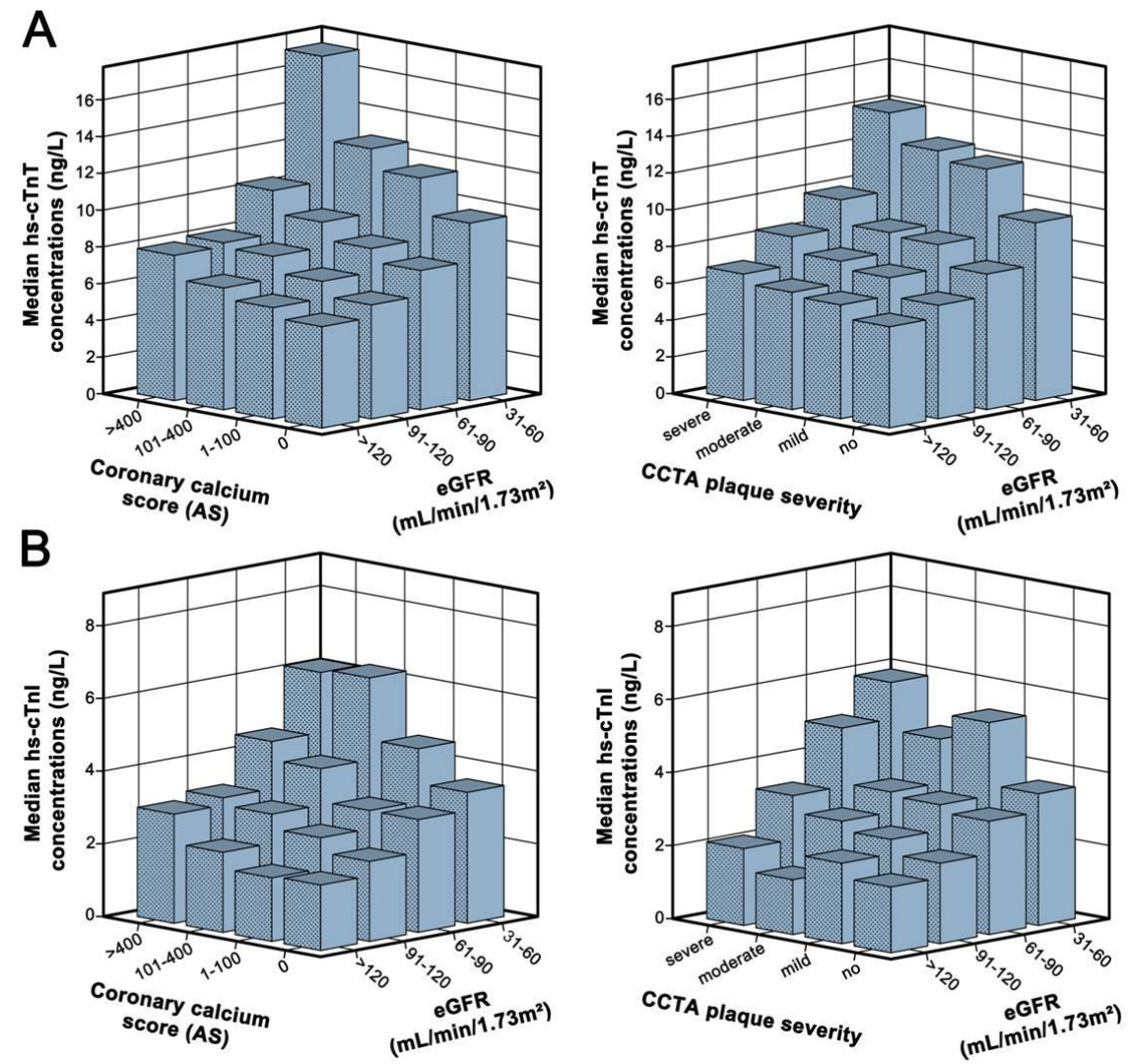

FIGURE 1. Median hs-cTnT (A) and hs-cTnl (B) concentrations according to estimated GFR categories and coronary calcium score or CCTA plaque severity. AS, agatston score

Similar standardized $\beta_{\text {eGFR }}\left(s t \beta_{\text {eGFR }}\right)$, st $\beta_{\text {CCS }}$ and st $\beta_{\text {CCTA }}$ coefficients were observed in the univariable versus unadjusted multivariable models, indicating that CT parameters were hardly influenced by the addition of eGFR when predicting hs-cTnT or hs-cTnl concentrations (TABLE 2). After adjustment for traditional cardiovascular risk factors, eGFR (hs-cTnT: st $\beta$ :-0.289; hs-cTnl: st $\beta$ :0.222; p<0.001), CCS (hs-cTnT:st $\beta: 0.078 ;$ hs-cTnl:st $\beta: 0.092 ; p<0.001$ ) and CCTA plaque severity score (hs-cTnT:st $\beta: 0.0 .074 ;$ hs-cTnl:st $\beta: 0.072 ; p<0.001$ ) remained significantly associated with hs-cTn concentrations (TABLE 2, adjusted multivariable model 1 and 2 , respectively). Also here, st $\beta_{\text {eGFR }}$ values remain unchanged (TABLE 2 , adjusted multivariable models 1 and 3 ). 


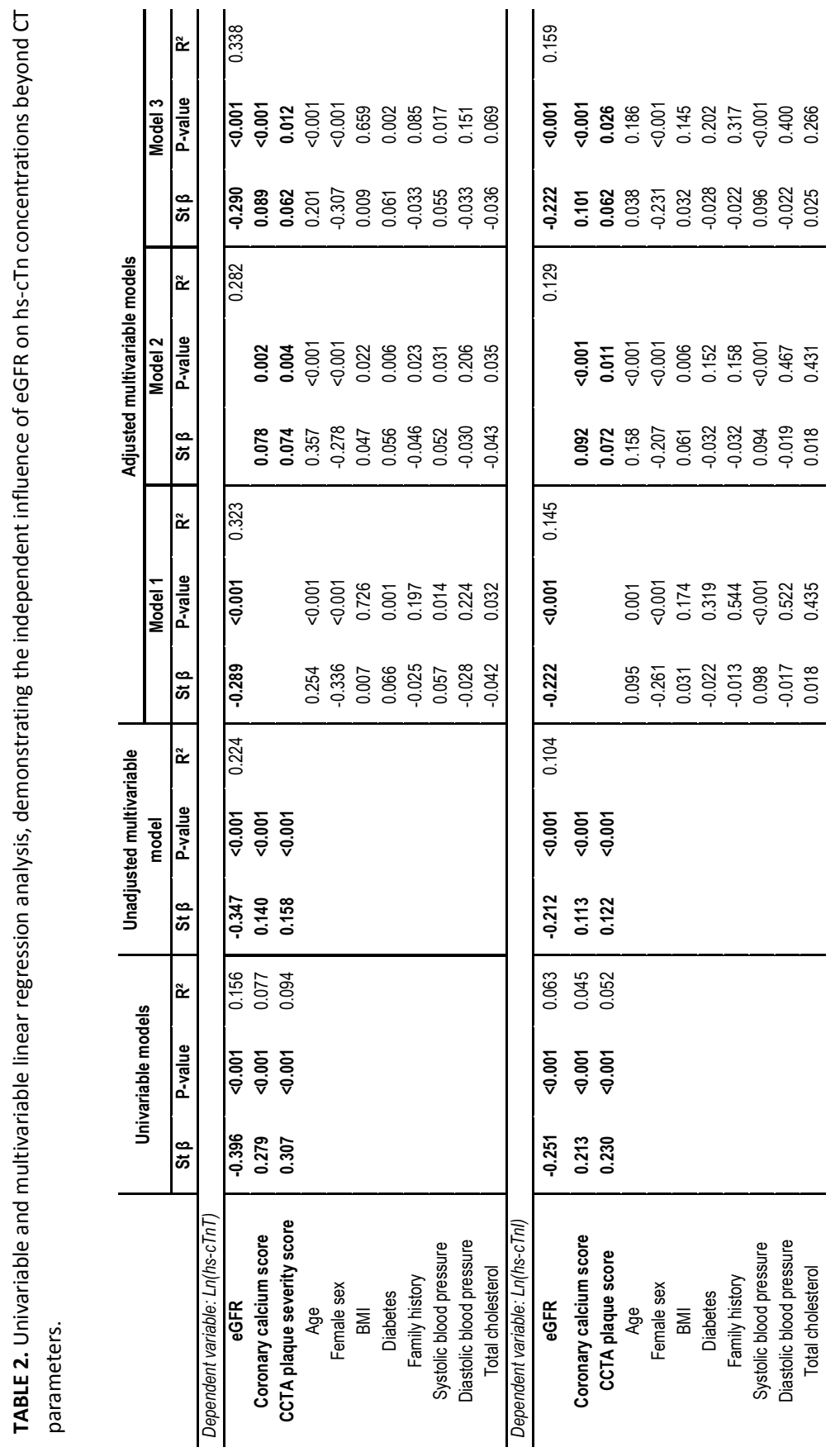


The independent contribution of eGFR to the prediction of hs-cTnT and hs-cTnl concentrations was also demonstrated by significant and identical $R^{2}$ changes (hs-cTnT: 0.056, hs-cTnl: 0.030; all p<0.001) when adding eGFR either to the baseline model or to adjusted multivariable model 2 .

Furthermore, the association of eGFR with hs-cTn concentrations remained equally strong when subdividing this cohort into patients with no (hscTnT:st $\beta_{\text {eGFR }}:-0.295$; hs-cTnl:st $\beta_{\text {eGFR }}:-0.228$ ), mild (hs-cTnT:st $\beta_{\text {eGFR }}:-0.290$; hscTnl:st $\beta_{\text {eGFR }}:-0.176$ ) and moderate-to-severe CAD (hs-cTnT:st $\beta_{\text {eGFR }}:-0.293$;hs-

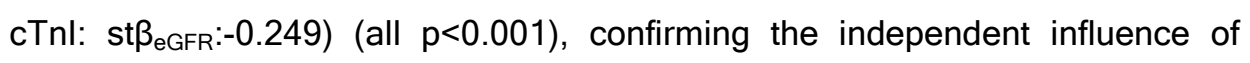
eGFR on hs-cTn concentrations beyond CAD severity (TABLE 3 ). This finding was visible but less apparent for hs-cTnl than for hs-cTnT.

Considering the hs-cTnT concentrations above the $99^{\text {th }}$ percentile concentrations, we found that $47 \%$ were suffering from significant CAD (presence of obstructive plaques or CCS > 400) and $53 \%$ had decreased renal function (eGFR $<90 \mathrm{~mL} / \mathrm{min} / 1.73 \mathrm{~m}^{2}$ ). Moreover, $72 \%$ of the patients with elevated hs-cTnT concentrations were diagnosed with CAD or had a decreased eGFR (SUPPLEMENTAL TABLE 2). For hs-cTnl, this was $43 \%, 30 \%$ and $58 \%$, respectively. Similar findings were observed when applying gender-specific cutoffs (data not shown).

Comparable regression analyses were performed including echocardiographic parameters as explanatory variables for hs-cTn concentrations. Univariably, hscTnT and hs-cTnl were significantly associated with the echocardiographic measures LVEF (hs-cTnT: R:-0.151, $p=0.001$; hs-cTnl, R:-0.142, $p=0.002$ ) and LVmass (hs-cTnT: R:0.253; hs-cTnl: R:0.309; p<0.001) (SUPPLEMENTAL TABLE 3 and SUPPLEMENTAL FIGURE 3). In line with previous results, st $\beta_{\text {eGFR }}$ coefficients were only influenced by the confounding effects of traditional cardiovascular risk factors and not by any of the measured CT parameters or echocardiographic parameters (SUPPLEMENTAL TABLE 4).

In total, $30 \%$ and $19 \%$ of hs-cTnT and hs-cTnl variation, respectively, could be explained by the most important independent predictors: eGFR, CCS, LVmass, age and male sex (SUPPLEMENTAL TABLE 4). 


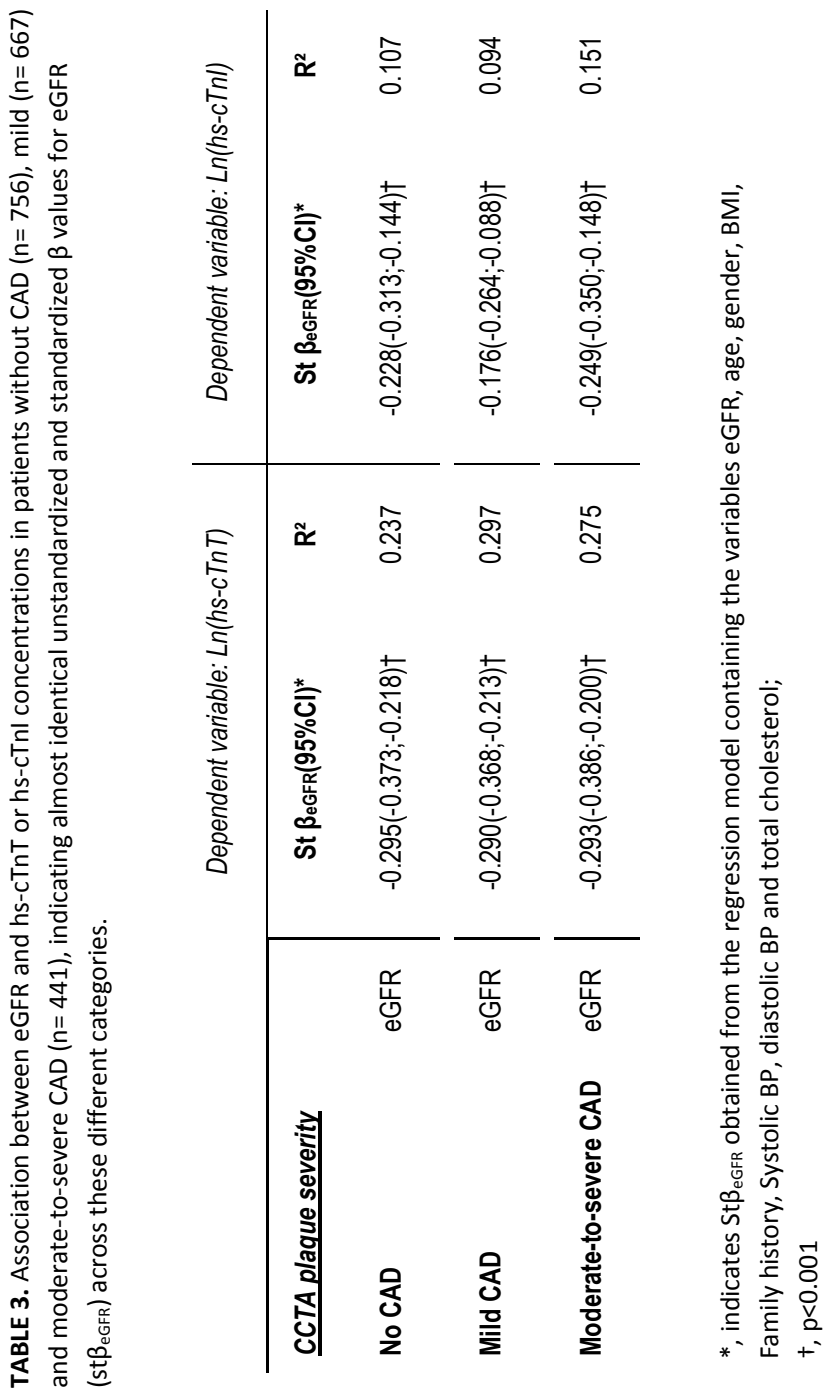




\section{Algorithm between eGFR and hs-cTn concentrations}

In patients with an eGFR between $120-130 \mathrm{~mL} / \mathrm{min} / 1.73 \mathrm{~m}^{2}$, median hs-cTnT and hs-cTnl concentrations were $6.0 \mathrm{ng} / \mathrm{L}$ and $2.0 \mathrm{ng} / \mathrm{L}$, respectively (TABLE 4). Moreover, when eGFR was halved to $60 \mathrm{~mL} / \mathrm{min} / 1.73 \mathrm{~m}^{2}$, a doubling of both hscTn concentrations was observed (hs-cTnT: $9.9 \mathrm{ng} / \mathrm{L}$; hs-cTnl: $4.1 \mathrm{ng} / \mathrm{L}$ ). A decrease in eGFR of $10 \mathrm{~mL} / \mathrm{min} / 1.73 \mathrm{~m}^{2}$ corresponded in this population to a mean relative increase of $9 \%$ and $11 \%$ or mean absolute increase of $0.8 \mathrm{ng} / \mathrm{L}$ and $0.4 \mathrm{ng} / \mathrm{L}$ in hs-cTnT and hs-cTnl concentrations, respectively.

\section{Hs-cTn remain significant and comparable prognostic markers for cardiovascular events}

Over a mean follow-up period of $2.9 \pm 1.5$ years, 54 cardiovascular events $(3.2 \%)$ were observed, encompassing 33 patients who underwent late revascularization, 18 patients that suffered from a non-fatal acute coronary syndrome and 3 patients who died due to cardiac disease (decompensatio cordis and myocardial infarction) or vascular causes (cerebrovascular attack). From all traditional risk factors, only age, gender and smoking were significantly different in the event versus non-event group (SUPPLEMENTAL TABLE 5).

As depicted in FIGURE 2, univariable Cox regression analysis showed that the incidence of cardiovascular events was significantly associated with hs-cTnT and hs-cTnl. Moreover, both hs-cTnT and hs-cTnl remained highly significant predictors for cardiovascular events when adjusted for eGFR and CCS or CCTA plaque severity .

ROC analysis demonstrated no significant difference between hs-cTnT (areaunder-the-curve, AUC (95\% confidence intervals): 0.671(0.599-0.742)) and hscTnl $(0.649(0.571-0.727))$ in the prediction of cardiovascular events $(p>0.05$, data not shown). 


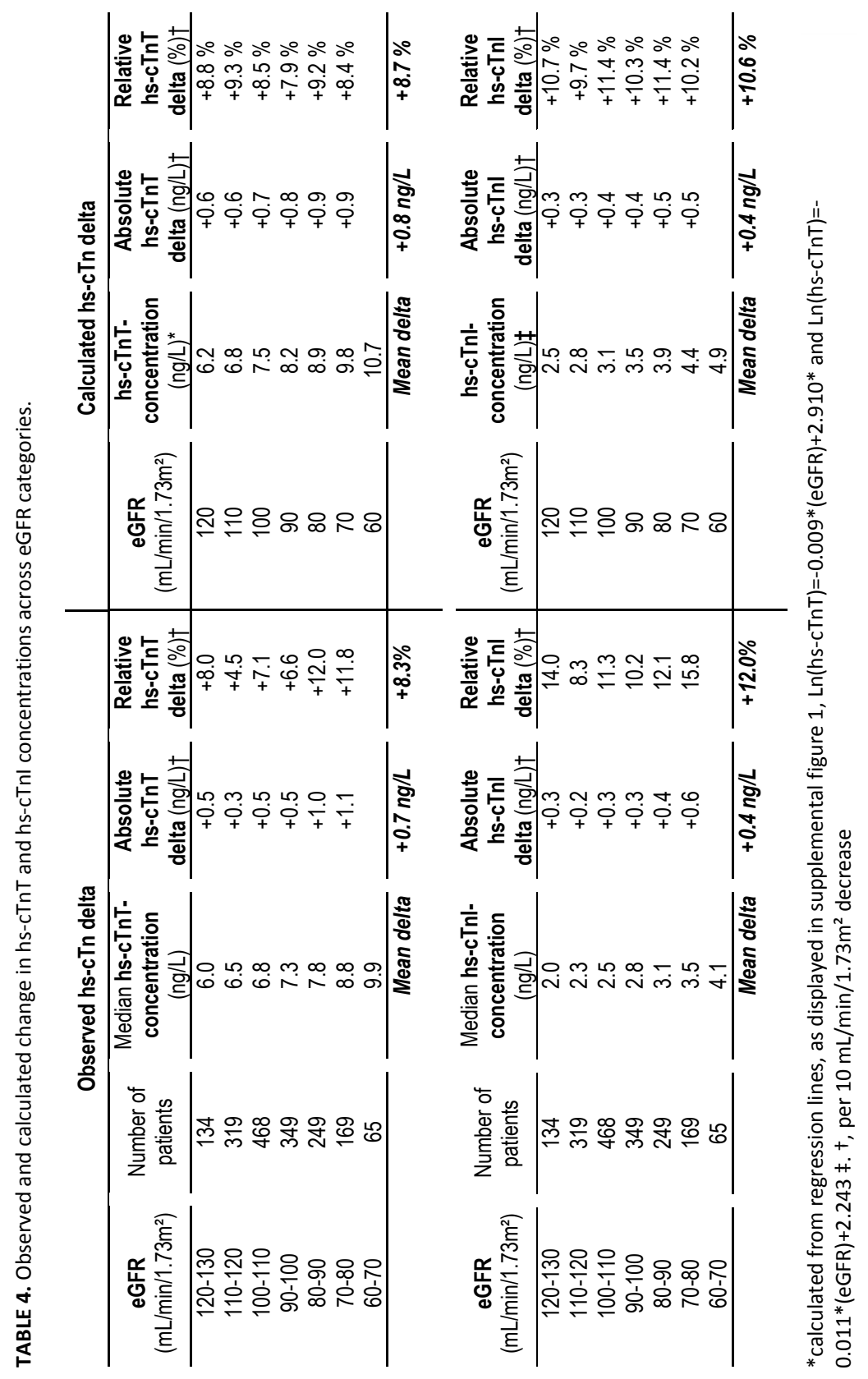



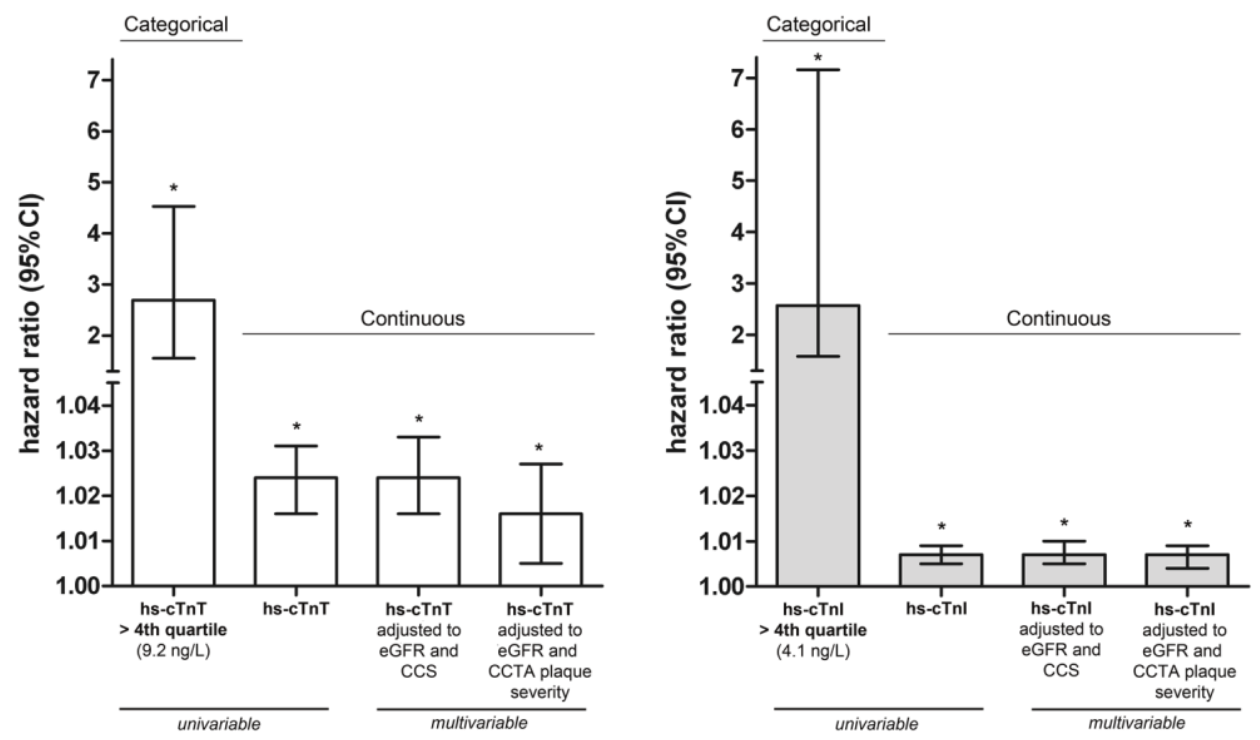

FIGURE 2. Hazard ratios and $95 \%$ confidence intervals $(\mathrm{Cl})$ for cardiovascular events as predicted by hs-cTnT or hs-cTnl either unadjusted or adjusted to eGFR and the cardiac imaging parameters: coronary calcium score (CCS) and CCTA plaque severity score. ${ }^{*}$, indicates a p-value $<0.001$

\section{Discussion}

The present study provides new insights into the interpretation of hs-cTn concentrations in patients with chest discomfort, identifying renal function and CAD as independent and strong contributors to circulating hs-cTn concentrations. In fact, in this population eGFR exhibited limited confounding effects on the association between hs-cTn and cardiac parameters.

\section{Significant influence of renal function on hs-cTnT and hs-cTn/ concentrations}

We found that hs-cTnT and hs-cTnl were strongly correlated with eGFR in patients with chest discomfort, in which $98 \%$ had a renal function $>60 \mathrm{~mL} / \mathrm{min} / 1.73 \mathrm{~m}^{2}$. In this cohort, a decrease in eGFR of $10 \mathrm{~mL} / \mathrm{min} / 1.73 \mathrm{~m}^{2}$ indicated a $\pm 10 \%$ increase in hs-cTn concentrations, equivalent to an absolute increase of $0.8 \mathrm{ng} / \mathrm{L}$ and $0.4 \mathrm{ng} / \mathrm{L}$ for hs-cTnT and hs-cTnl, respectively. Although these findings need to be validated in other populations, it clearly 
illustrates that a decreased renal clearance, even within normal boundaries, can affect hs-cTn concentrations and questions whether baseline hs-cTn concentrations should be reported without the access to an eGFR.

As observed in moderate-to-severe CKD patients [13], hs-cTnT was more strongly correlated with eGFR than hs-cTnl. In addition, the association between hs-cTnT concentrations and eGFR were stronger than any known association between hs-cTnT and cardiac parameters, such as coronary plaque severity $[2,3,27]$ or left ventricular structure $[16,18,28]$. Interestingly, this is in contrast to hs-cTnl, in which the association with LVmass was greater than with eGFR. These findings suggest that hs-cTnT could be more susceptible to changes in renal clearance than hs-cTnl.

\section{Renal and cardiac parameters as two separate contributors to hs-cTn}

Importantly, in a cohort of patients with normal eGFR values, we demonstrate for the first time that the extent of CAD and echocardiographic characteristics of the left ventricle did not interfere with the magnitude of the association between eGFR and hs-cTn concentrations. As a consequence, in patients with a normal to a mildly impaired kidney function, decreases in renal function and the extent of CAD can be considered as two separate contributors of hs-cTn accumulation. This independent association with renal function might not only be valuable for the evaluation of stable chest pain patients [1] but also chronic heart failure and acute chest pain patients [4].

Furthermore, we show that a significant amount of the patients with elevated hscTn concentrations suffered from decreased renal function, besides the presence of CAD. These findings can explain the reduced diagnostic performance of hs-cTn seen in AMl patients with lower renal function in comparison to those with normal renal function [29, 30]. Further research should therefore focus on the influence of mild CKD when acquiring the diagnostic hscTn cutoffs as established from reference populations $\left(99^{\text {th }}\right.$ upper reference limit).

The significant association between eGFR and hs-cTn concentrations, independent from cardiac pathologies, is in line with the observation that CTnT and $\mathrm{cTnl}$ are cleared by the kidneys [31, 32]. However, we cannot exclude that a 
decreased renal function may exert additional myocardial stress that is not identified by cardiac imaging, leading to subsequent cardiac troponin release.

\section{Prognostic importance of hs-cTnT and hs-cTnl besides CCTA and renal function}

In this study, serum hs-cTnT as well as hs-cTnl were significant prognostic markers for the development of cardiovascular events, as previously demonstrated $[8,9,28,33]$. Although hs-cTnT concentrations were twice as high as hs-cTnl concentrations, we found the overall prognostic performance of hs-cTnT and hs-cTnl to be comparable $\left(A_{\mathrm{U}} \mathrm{C}_{\mathrm{ROC}}\right.$ : 0.670 versus 0.650 for hscTnT and hs-cTnl, respectively).

Hs-cTnT was shown to be a strong prognostic marker for cardiovascular events, despite the influence of a decreased renal function [34, 35]. We found that even when adjusted to eGFR, both hs-cTn concentrations still hold an important prognostic value besides important cardiovascular imaging parameters such as CCS and CCTA plaque severity score. In fact, we were able to explain a quarter to one third of the variability in hs-cTn concentrations, suggesting that other mechanisms could contribute to the overall hs-cTn level as well.

\section{Study limitations}

A limitation to our survival analysis is that this is a low-risk population and consequently a low number of adverse cardiovascular events were observed. Also, we cannot rule out that CCTA outcomes were used in the decision to perform a $\mathrm{CABG}$ and $\mathrm{PCl}$ in these patients. However, we corrected for this bias by excluding the procedures that were performed within 90 days after CCTA. Furthermore, urine samples were not available for the assessment of albuminuria.

\section{Conclusion}

In conclusion, we identified in patients with symptoms of chest discomfort that renal function has a significant influence on circulating hs-cTnT and hs-cTnl concentrations. Moreover, we found that cardiac pathology and decreases in renal function are two independent contributors for hs-cTn accumulation. Prospective studies must establish whether baseline hs-cTn concentrations 
should be accompanied by GFR-assessment. Notwithstanding, hs-cTnT and hscTnl remained significant predictors of cardiovascular events, even on top of cardiovascular disease and renal function.

\section{Acknowledgments}

We sincerely thank Abbott Diagnostics for providing the hs-cTnl kits. The authors also acknowledge the contribution of dr. L. Hofstra to the CT study and the laboratory assistance of V.W.V.C. Kleijnen. This work was supported by a research grant from Stichting De Weijerhorst to M.P v.D-V. 


\section{References}

1. ESC Task Force Members, 2013 ESC guidelines on the management of stable coronary artery disease: the Task Force on the management of stable coronary artery disease of the European Society of Cardiology. Eur Heart J, 2013. 34(38): p. 2949-3003.

2. Laufer, E.M., A.M. Mingels, M.H. Winkens, et al., The extent of coronary atherosclerosis is associated with increasing circulating levels of high sensitive cardiac troponin T. Arterioscler Thromb Vasc Biol, 2010. 30(6): p. 1269-75.

3. Korosoglou, G., S. Lehrke, D. Mueller, et al., Determinants of troponin release in patients with stable coronary artery disease: insights from CT angiography characteristics of atherosclerotic plaque. Heart, 2011. 97(10): p. 823-31.

4. Thygesen, K., J.S. Alpert, A.S. Jaffe, et al., Third universal definition of myocardial infarction. Circulation, 2012. 126(16): p. 2020-35.

5. Giannitsis, E., K. Kurz, K. Hallermayer, et al., Analytical validation of a high-sensitivity cardiac troponin T assay. Clin Chem, 2010. 56(2): p. 254-61.

6. Reichlin, T., W. Hochholzer, S. Bassetti, et al., Early diagnosis of myocardial infarction with sensitive cardiac troponin assays. N Engl J Med, 2009. 361(9): p. 858-67.

7. Alcalai, R., D. Planer, A. Culhaoglu, et al., Acute coronary syndrome vs nonspecific troponin elevation: clinical predictors and survival analysis. Arch Intern Med, 2007. 167(3): p. 276-81.

8. Bonaca, M., B. Scirica, M. Sabatine, et al., Prospective evaluation of the prognostic implications of improved assay performance with a sensitive assay for cardiac troponin I. J Am Coll Cardiol, 2010. 55(19): p. 2118-24.

9. Omland, T., M.A. Pfeffer, S.D. Solomon, et al., Prognostic value of cardiac troponin I measured with a highly sensitive assay in patients with stable coronary artery disease. J Am Coll Cardiol, 2013. 61(12): p. 1240-9.

10. Giannitsis, E. and H.A. Katus, Cardiac troponin level elevations not related to acute coronary syndromes. Nat Rev Cardiol, 2013. 10(11): p. 623-34.

11. deFilippi, C., S. Wasserman, S. Rosanio, et al., Cardiac troponin T and C-reactive protein for predicting prognosis, coronary atherosclerosis, and cardiomyopathy in patients undergoing long-term hemodialysis. JAMA, 2003. 290(3): p. 353-9.

12. Freda, B.J., W.H. Tang, F. Van Lente, et al., Cardiac troponins in renal insufficiency: review and clinical implications. J Am Coll Cardiol, 2002. 40(12): p. 2065-71.

13. deFilippi, C., S.L. Seliger, W. Kelley, et al., Interpreting cardiac troponin results from highsensitivity assays in chronic kidney disease without acute coronary syndrome. Clin Chem, 2012. 58(9): p. 1342-51.

14. Bansal, N., A. Hyre Anderson, W. Yang, et al., High-Sensitivity Troponin T and N-Terminal Pro-B-Type Natriuretic Peptide (NT-proBNP) and Risk of Incident Heart Failure in Patients with CKD: The Chronic Renal Insufficiency Cohort (CRIC) Study. J Am Soc Nephrol, 2014.

15. Dubin, R.F., Y. Li, J. He, et al., Predictors of high sensitivity cardiac troponin T in chronic kidney disease patients: a cross-sectional study in the chronic renal insufficiency cohort (CRIC). BMC Nephrol, 2013. 14: p. 229.

16. Mishra, R.K., Y. Li, C. DeFilippi, et al., Association of cardiac troponin T with left ventricular structure and function in CKD. Am J Kidney Dis, 2013. 61(5): p. 701-9.

17. Chin, C.W., A.S. Shah, D.A. McAllister, et al., High-sensitivity troponin I concentrations are a marker of an advanced hypertrophic response and adverse outcomes in patients with aortic stenosis. Eur Heart J, 2014.

18. Rosjo, H., J. Andreassen, T. Edvardsen, et al., Prognostic usefulness of circulating highsensitivity troponin $\mathrm{T}$ in aortic stenosis and relation to echocardiographic indexes of cardiac function and anatomy. Am J Cardiol, 2011. 108(1): p. 88-91.

19. Beatty, A.L., I.A. Ku, R.H. Christenson, et al., High-sensitivity cardiac troponin T levels and secondary events in outpatients with coronary heart disease from the Heart and Soul Study. JAMA Intern Med, 2013. 173(9): p. 763-9. 
20. Joosen, I.A., F. Schiphof, M.O. Versteylen, et al., Relation between mild to moderate chronic kidney disease and coronary artery disease determined with coronary CT angiography. PLoS One, 2012. 7(10): p. e47267.

21. Voskoboev, N.V., T.S. Larson, A.D. Rule, et al., Importance of cystatin C assay standardization. Clin Chem, 2011. 57(8): p. 1209-11.

22. Inker, L.A., C.H. Schmid, H. Tighiouart, et al., Estimating glomerular filtration rate from serum creatinine and cystatin C. N Engl J Med, 2012. 367(1): p. 20-9.

23. Agatston, A.S., W.R. Janowitz, F.J. Hildner, et al., Quantification of coronary artery calcium using ultrafast computed tomography. J Am Coll Cardiol, 1990. 15(4): p. 827-32.

24. Lang, R.M., M. Bierig, R.B. Devereux, et al., Recommendations for chamber quantification. Eur J Echocardiogr, 2006. 7(2): p. 79-108.

25. Galiuto, L., L. Badano, K. Fox, et al., The EAE Textbook of Echocardiography. 2011: Oxford university press.

26. Mingels, A.M., I.A. Joosen, M.O. Versteylen, et al., High-sensitivity cardiac troponin T: risk stratification tool in patients with symptoms of chest discomfort. PLoS One, 2012. 7(4): p. e35059.

27. Januzzi, J.L., Jr., F. Bamberg, H. Lee, et al., High-sensitivity troponin T concentrations in acute chest pain patients evaluated with cardiac computed tomography. Circulation, 2010. 121(10): p. 1227-34.

28. de Lemos, J.A., M.H. Drazner, T. Omland, et al., Association of troponin T detected with a highly sensitive assay and cardiac structure and mortality risk in the general population. JAMA, 2010. 304(22): p. 2503-12.

29. Reichlin, T., A. Irfan, R. Twerenbold, et al., Utility of absolute and relative changes in cardiac troponin concentrations in the early diagnosis of acute myocardial infarction. Circulation, 2011. 124(2): p. 136-45.

30. Chenevier-Gobeaux, C., C. Meune, Y. Freund, et al., Influence of age and renal function on high-sensitivity cardiac troponin T diagnostic accuracy for the diagnosis of acute myocardial infarction. Am J Cardiol, 2013. 111(12): p. 1701-7.

31. Ziebig, R., A. Lun, B. Hocher, et al., Renal elimination of troponin T and troponin I. Clin Chem, 2003. 49(7): p. 1191-3.

32. Tsutamoto, T., C. Kawahara, M. Yamaji, et al., Relationship between renal function and serum cardiac troponin T in patients with chronic heart failure. Eur J Heart Fail, 2009. 11(7): p. 653-8.

33. Omland, T., J.A. de Lemos, M.S. Sabatine, et al., A sensitive cardiac troponin T assay in stable coronary artery disease. N Engl J Med, 2009. 361(26): p. 2538-47.

34. Scheven, L., P.E. de Jong, H.L. Hillege, et al., High-sensitive troponin T and N-terminal pro-B type natriuretic peptide are associated with cardiovascular events despite the crosssectional association with albuminuria and glomerular filtration rate. Eur Heart J, 2012. 33(18): p. 2272-81.

35. Hassan, H.C., K. Howlin, A. Jefferys, et al., High-sensitivity troponin as a predictor of cardiac events and mortality in the stable dialysis population. Clin Chem, 2014. 60(2). 

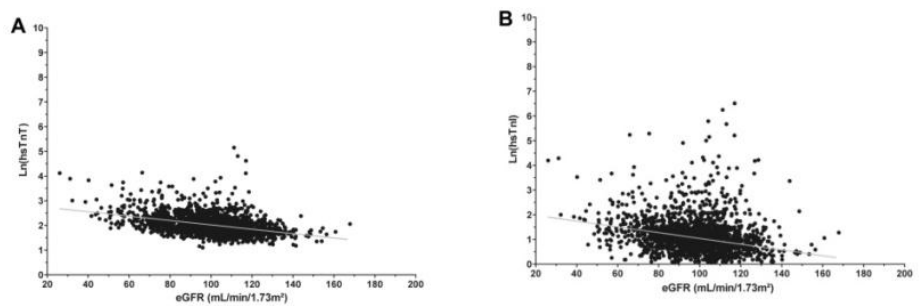

SUPPLEMENTAL FIGURE 1. Scatterplots of the association of eGFR with Ln (hs-cTnT) (A) and Ln (hs-cTnl) (B).

A

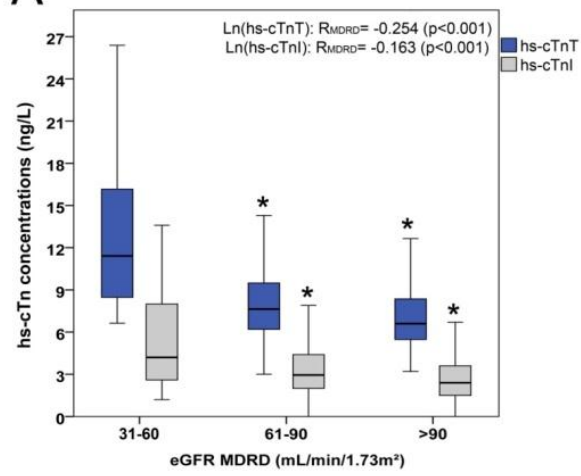

C

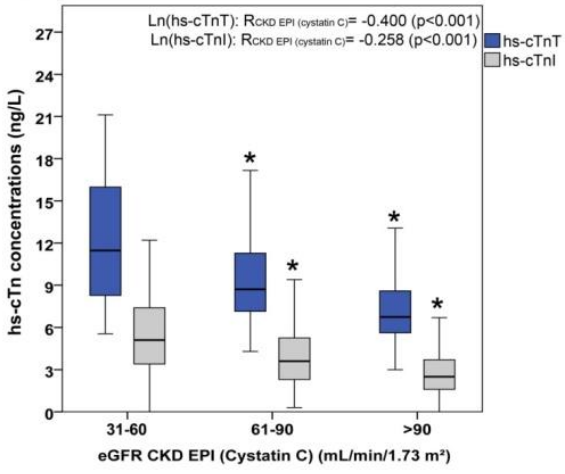

B

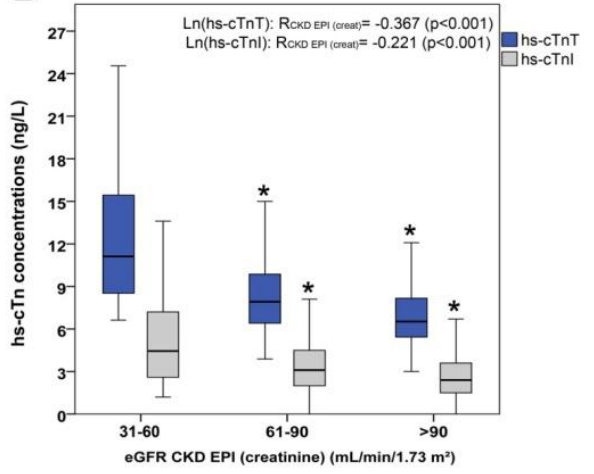

D

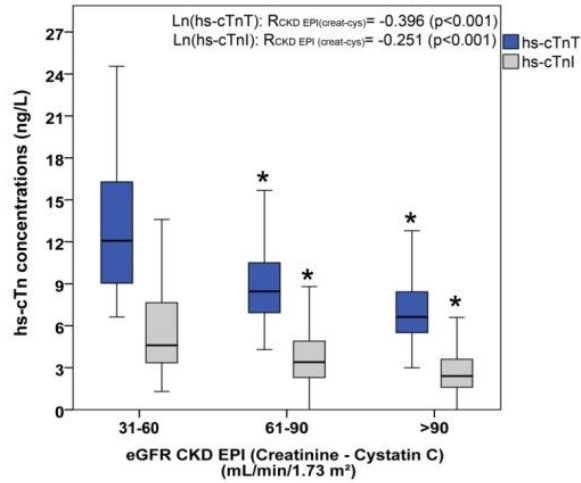

SUPPLEMENTAL FIGURE 2. Association of hs-cTnT (blue) and hs-cTnl (grey) with GFR estimated by the MDRD equation $(A)$ and the CKD EPI equations using serum creatinine $(B)$, serum cystatin $C(C)$ concentrations or both (D). *p-value $<0.05$ in comparison with eGFR 31-60 ml/min/1.73 m2 group. Pearson R correlation factors with the natural logarithm of hs-cTnT and hs-cTnl are given. 
A

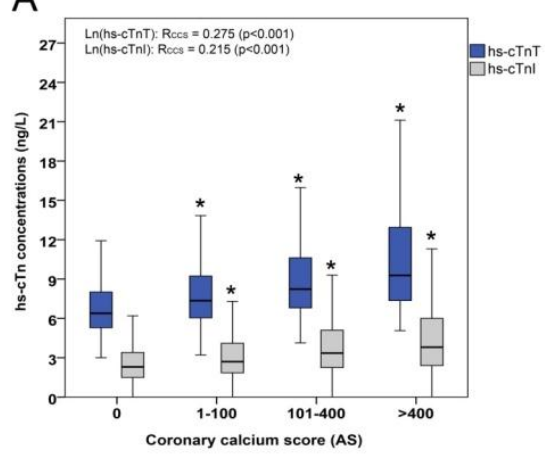

C

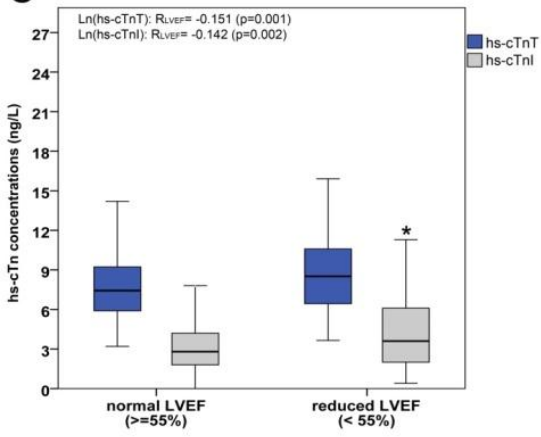

B

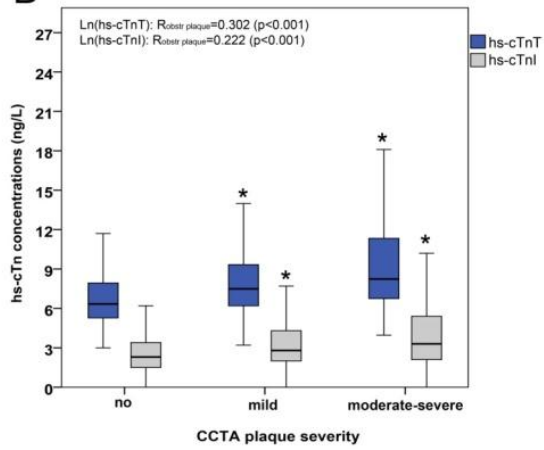

D

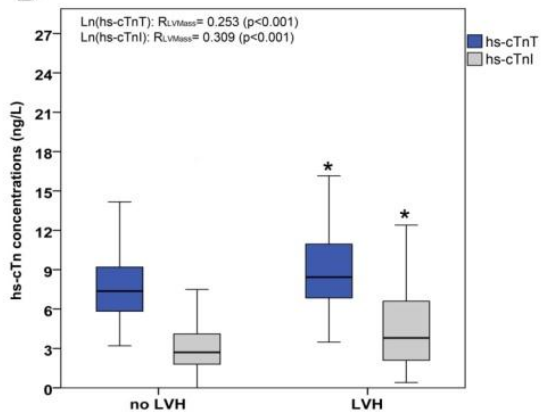

SUPPLEMENTAL FIGURE 3. Association of hs-cTnT (blue) and hs-cTnl (grey) with the coronary calcium score (CCS) (A), CCTA plaque severity (B), and echocardiographic parameters, LVEF (C) and LVH (D). * $p$-value $<0.05$ in comparison with the non-diseased group. Pearson $\mathrm{R}$ correlation factors with the natural logarithm of hscTnT and hs-cTnl are given. AS, indicates Agatston score 

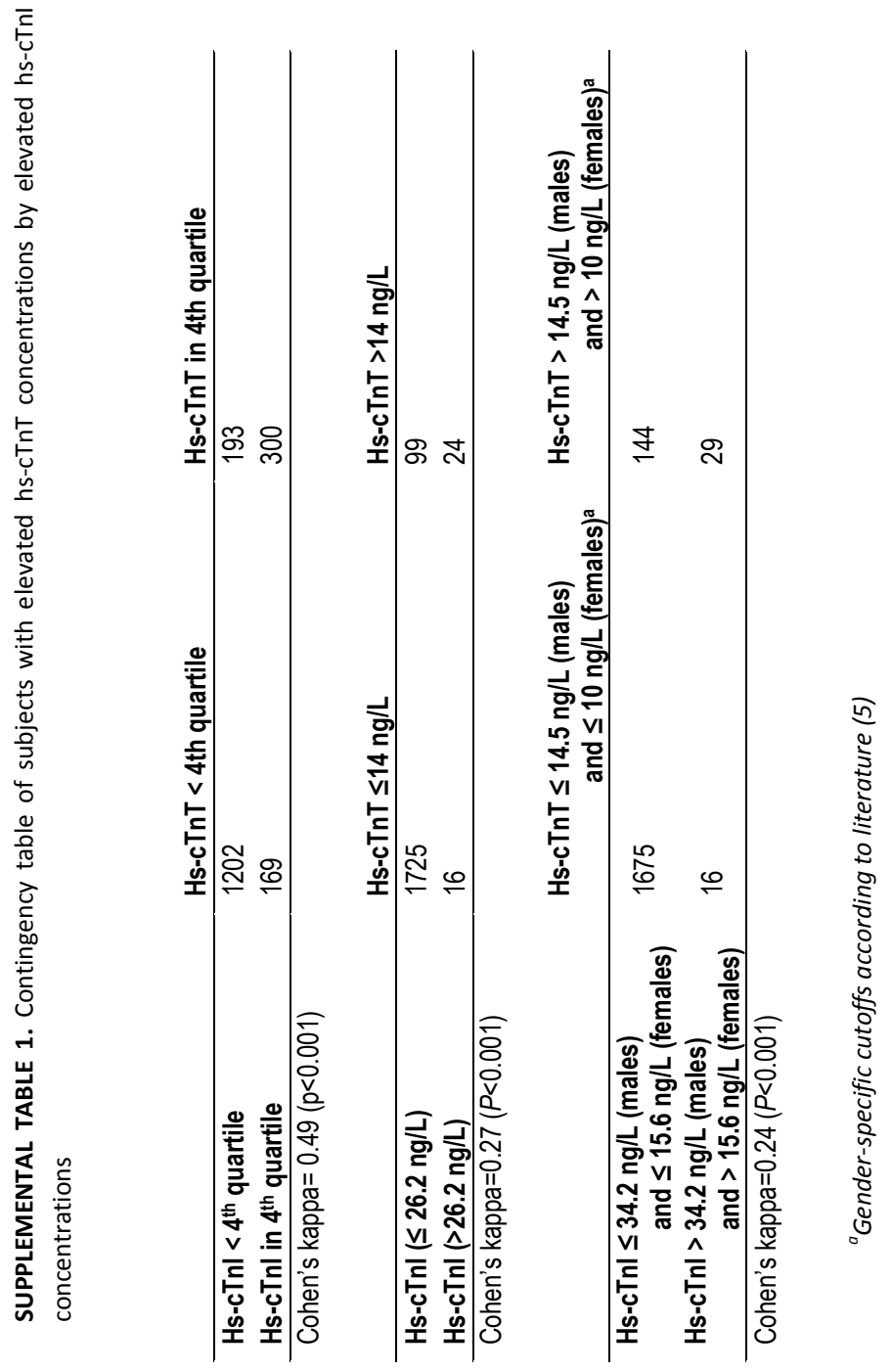
SUPPLEMENTAL TABLE 2. The presence (\%) of CAD and renal dysfunction in patients with elevated hs-cTnT and hs-cTnl concentrations.

\begin{tabular}{|c|c|c|c|c|}
\hline & \multicolumn{2}{|c|}{$\begin{array}{l}\text { Patients with hs-cTn > } \\
\text { 99th percentile cutoff }\end{array}$} & \multicolumn{2}{|c|}{$\begin{array}{l}\text { Patients with hs-cTn > } \\
\text { gender specific } 99 \text { th percentile cutoff }\end{array}$} \\
\hline & Hs-cTnT (n=123) & Hs-cTnl $(n=40)$ & Hs-cTnT (n=173) & Hs-cTnl $(n=45)$ \\
\hline & Percentage (n) & Percentage (n) & Percentage (n) & Percentage (n) \\
\hline Obstructive plaque & $42 \%(52 / 123)$ & $38 \%(15 / 40)$ & $39 \%(68 / 173)$ & $36 \%(16 / 45)$ \\
\hline $\begin{array}{l}\text { Obstructive plaque } \\
\text { or CCS }>400\end{array}$ & $47 \%(58 / 123)$ & $43 \%(17 / 40)$ & $43 \%(75 / 173)$ & $42 \%(19 / 45)$ \\
\hline eGFR $<90 \mathrm{~mL} / \mathrm{min} / 1.73 \mathrm{~m}^{2}$ & $53 \%(65 / 123)$ & $30 \%(12 / 40)$ & $61 \%(105 / 173)$ & $27 \%(12 / 45)$ \\
\hline $\begin{array}{c}\text { Obstructive plaque } \\
\text { or CCS }>400 \text { or } \\
\text { eGFR }<90 \mathrm{~mL} / \mathrm{min} / 1.73 \mathrm{~m}^{2}\end{array}$ & $72 \%(88 / 123)$ & $58 \%(23 / 40)$ & $75 \%(129 / 173)$ & $56 \%(25 / 45)$ \\
\hline
\end{tabular}

SUPPLEMENTAL TABLE 3. Baseline characteristics of subpopulation ( $N=549 / 1876$ patients) in which echocardiography was performed, by median hs-cTnT and hs-cTnl concentrations.

\begin{tabular}{|c|c|c|c|c|c|c|c|}
\hline \multirow[b]{2}{*}{ Determinant } & \multirow[b]{2}{*}{$\begin{array}{c}\text { All } \\
\text { patients }\end{array}$} & \multicolumn{2}{|c|}{ hs-cTnT } & \multirow[b]{2}{*}{$\begin{array}{c}\mathrm{P} \text { - } \\
\text { value }\end{array}$} & \multicolumn{2}{|c|}{ hs-cTnl } & \multirow[b]{2}{*}{$\begin{array}{l}\mathrm{P}- \\
\text { value }\end{array}$} \\
\hline & & $\begin{array}{l}\leq 7.2 \mathrm{ng} / \mathrm{L} \\
(\mathrm{N}=231)\end{array}$ & $\begin{array}{l}>7.2 \mathrm{ng} / \mathrm{L} \\
(\mathrm{N}=318)\end{array}$ & & $\begin{array}{l}\leq 2.6 \mathrm{ng} / \mathrm{L} \\
(\mathrm{N}=163)\end{array}$ & $\begin{array}{l}>2.6 \mathrm{ng} / \mathrm{L} \\
(\mathrm{N}=386)\end{array}$ & \\
\hline $\begin{array}{l}\text { Left ventricular } \\
\text { ejection fraction,\% }\end{array}$ & $60.0 \pm 7.3$ & $60.7 \pm 5.9$ & $59.4 \pm 8.2$ & 0.037 & $60.8 \pm 5.5$ & $59.6 \pm 8.0$ & 0.056 \\
\hline $\begin{array}{l}\text { Left ventricular } \\
\text { mass, } \mathrm{g}\end{array}$ & $169.3 \pm 45.0$ & $158 \pm 37$ & $178 \pm 49$ & $<0.001$ & $155 \pm 34$ & $176 \pm 48$ & $<0.001$ \\
\hline $\begin{array}{l}\text { Left ventricular } \\
\text { mass index, } \mathrm{g} / \mathrm{m}^{2}\end{array}$ & $87.4 \pm 20.0$ & $83.2 \pm 16.6$ & $90.7 \pm 21.8$ & $<0.001$ & $82.5 \pm 16.1$ & $89.7 \pm 21.2$ & $<0.001$ \\
\hline
\end{tabular}



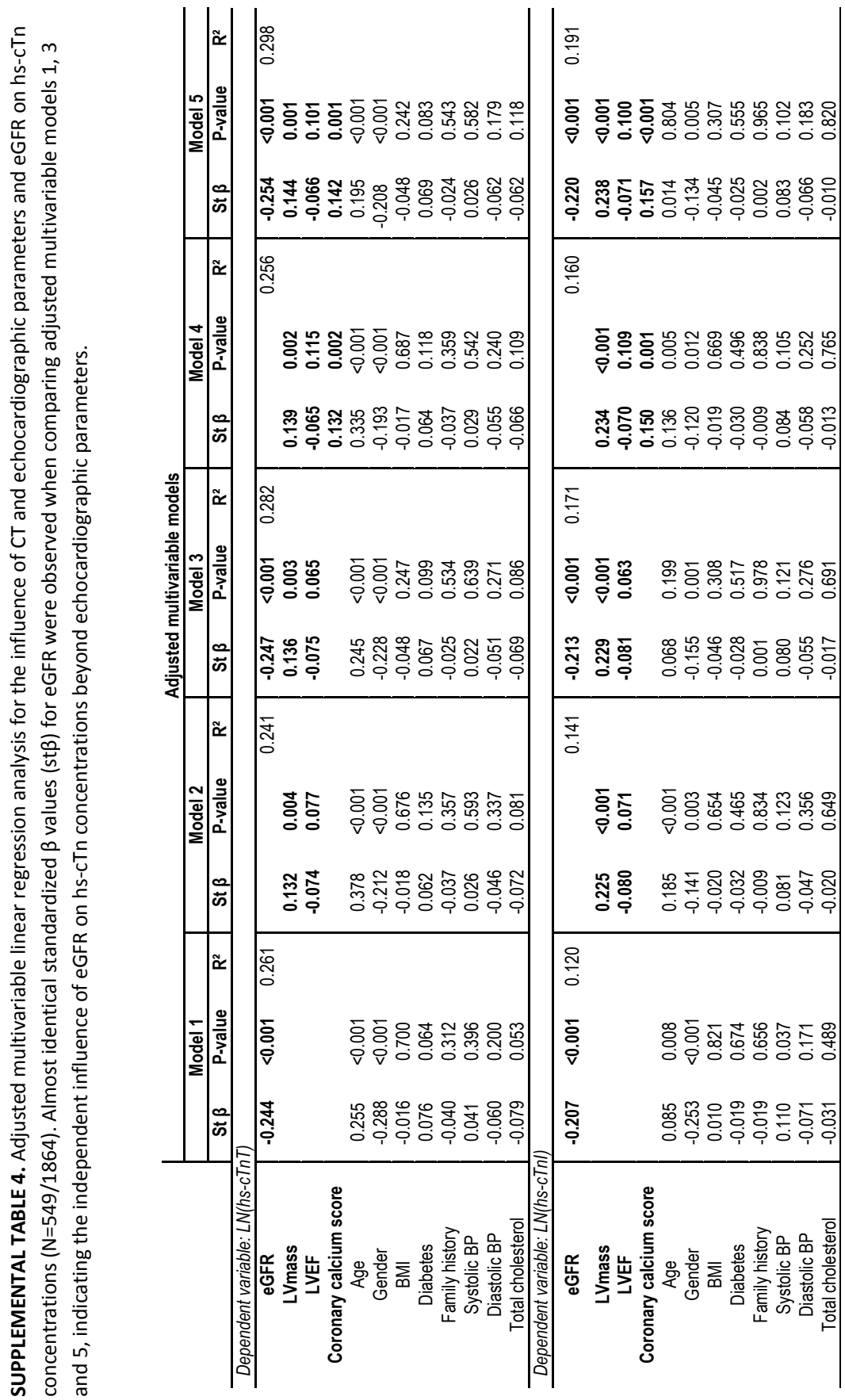
SUPPLEMENTAL TABLE 5. Differences in baseline characteristics between cardiovascular event and eventfree group.

\begin{tabular}{lccc} 
Determinant & Event (N=54) & No event (N=1635) & P-value \\
\hline \multicolumn{1}{c}{ Traditional risk factors } & & & \\
\hline Males, $n(\%)$ & $39(72.2 \%)$ & $904(55.3 \%)$ & 0.014 \\
Age, years & $59.7 \pm 10.7$ & $56.4 \pm 10.8$ & 0.010 \\
BMl, kg/m & $26.6 \pm 3.5$ & $27.0 \pm 4.4$ & 0.586 \\
Smokers, $n(\%)$ & $39.7 \%$ & $21.7 \%$ & $<0.001$ \\
Diabetes, $\mathrm{n}(\%)$ & $7(13.0 \%)$ & $121(7.4 \%)$ & 0.129 \\
Fam. history, $\mathrm{n}(\%)$ & $17(31.5 \%)$ & $627(38.3 \%)$ & 0.307 \\
Systolic BP, mmHg & $146.0 \pm 17.5$ & $142.7 \pm 19.6$ & 0.147 \\
Diastolic BP, mmHg & $80.5 \pm 11.7$ & $80.0 \pm 11.4$ & 0.930 \\
Total cholesterol, mmol/L & $5.2 \pm 1.4$ & $5.5 \pm 1.2$ & 0.318 \\
\hline \multicolumn{1}{c}{ Cardiac biochemical markers } & & \\
\hline hs-cTnT, ng/L & $8.9(6.5)$ & $7.2(3.3)$ & 0.004 \\
hs-cTnl, ng/L & $3.6(7.4)$ & $2.7(2.4)$ & 0.024 \\
\hline \multicolumn{1}{c}{ Renal clearance } & & \\
\hline Cystatin C, mg/L & $0.86 \pm 0.27$ & $0.77 \pm 0.18$ & 0.006 \\
Creatinine, $\mu$ mol/L & $82.8 \pm 21.8$ & $74.8 \pm 15.6$ & 0.009 \\
eGFRcreat+cysc, mL/min/1.73m ${ }^{2}$ & $91.2 \pm 21.0$ & $98.6 \pm 18.3$ & 0.001 \\
\hline \multicolumn{1}{c}{ CT/CCTA parameters } & & \\
\hline Coronary calcium score, AS & $206.3(601.0)$ & $4.8(111.0)$ & $<0.001$ \\
Moderate-to-severe plaque, $\mathrm{n}(\%)$ & $40(74.1 \%)$ & $375(22.9 \%)$ & $<0.001$ \\
\hline
\end{tabular}






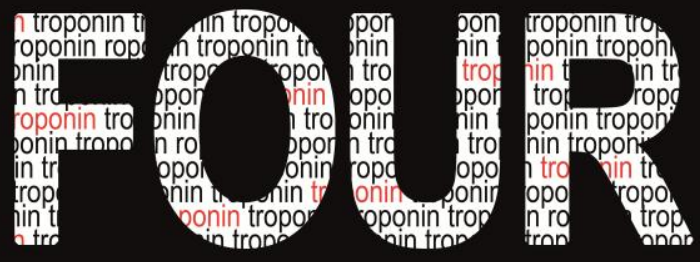

HIGH-SENSITIVITY CARDIAC TROPONIN T DIFFERS FROM TROPONIN I IN THE ASSOCIATION WITH RENAL DYSFUNCTION AND MORTALITY IN NURSING HOME RESIDENTS

Cardinaels E, Daamen M, Bekers O, ten Kate J, van Suijlen J, Niens M, van Dieijen-Visser M, Brunner-La Rocca HP, Schols J, Mingels A 


\section{Abstract}

\section{Background}

High-sensitivity cardiac troponins (hs-cTn) have proven to be valuable risk stratification tools in more general populations of older adults. In the present study we explore the value of measuring hs-cTn in geriatric care, by investigating the major determinants and prognostic importance of hs-cTnT and (hs)cTnl.

\section{Materials and methods}

495 residents from nursing homes (>65 years) of five healthcare organizations were examined. The residents underwent clinical and echocardiographic assessment for the diagnosis of heart failure. cTn was measured using highsensitivity (hs-)cTnT (Roche), hs-cTnl (Abbott) and less sensitive cTnl (Beckman) assays. The glomerular filtration rate was estimated (eGFR) using serum creatinine and cystatin C concentrations. Data on all-cause mortality were collected upon 1 year follow-up.

\section{Results}

Median (IQR) concentrations were 20.6(17.8-30.6) ng/L, 6.8(4.1-12.5) ng/L and 4.0(2.0-8.0) ng/L for hs-cTnT, hs-cTnl and cTnl respectively. In total, $79 \%$ had elevated hs-cTnT concentrations, while only $9 \%$ and $5 \%$ of hs-cTnl and cTnl concentrations were elevated. Most important determinants for higher hs-cTnT and hs-cTnl concentrations were of cardiac and renal origin. Whereas both heart failure (OR:3.4) and $\mathrm{eGFR}<60 \mathrm{~mL} / \mathrm{min} / 1.73 \mathrm{~m}^{2}$ (OR:3.6) were equal contributors to higher hs-cTnT concentrations (all p<0.001), hs-cTnl and cTnl were less associated to renal dysfunction (OR of respectively1.9 and 2.1; $\mathrm{p}<0.01$ ) in comparison to heart failure (OR:4.3 and 4.7, respectively, $p<0.001$ ). Still, higher hs-cTnT concentrations were a better predictor for all-cause mortality compared to both (hs-)cTnl assays.

\section{Conclusion}

In frail nursing home residents, all hs-cTn assays were highly associated with heart failure, while hs-cTnT was more associated with renal dysfunction than both (hs-)cTnl. Despite this, hs-cTnT had a more prominent role in assessing the risk on all-cause mortality. 


\section{Introduction}

Older adults receiving long-term care in nursing homes are thought to have a high risk on chronic diseases such as heart failure, but still remain a poorly investigated population [1, 2]. Predicting those at risk for chronic diseases and mortality can therefore lead to preventive strategies and enhancements in their quality of life.

Cardiac biomarkers, such as high-sensitivity cardiac troponins (hs-cTn) have proven to be valuable risk stratification tools in more general populations of older adults [3, 4] and are associated with the prevalence of cardiovascular diseases [5, 6]. Measuring hs-cTn is currently an essential component for the diagnosis of acute myocardial infarction (AMI) [7], in which the diagnostic cutoff is set at the $99^{\text {th }}$ percentile upper reference limit (URL) established in a healthy reference population [8,9]. Elevated hs-cTn concentrations are however commonly found in the elderly $[3,10,11]$ even when contributing comorbidities were excluded [12-15] and thereby challenging clinicians with the interpretation of these results [10]. These issues become even more important in frail older adults, such as nursing home residents. Therefore, age-specific cutoffs have been proposed $[5,10,11,15,16]$, although they are currently not implemented in the guidelines $[7,17]$.

In the present multicenter study on nursing home residents, we explore the value of measuring hs-cTnT and (hs-)cTnl in geriatric care. Major determinants and prognostic information of hs-cTn concentrations are evaluated in a wellcharacterized and representative cohort of nursing home residents over 65 years. Moreover, we examined whether higher cut-offs should be applied in these elderly and focus on the comparison between the results of hs-cTnT, hscTnl and a sensitive cTnl assay.

\section{Materials and methods}

\section{Study population}

In this study, 501 nursing home residents from five healthcare organizations in the Netherlands who receive long term care were examined. Residents were eligible when over 65 years of age, not receiving palliative care and not admitted 
for short-term rehabilitation (<2 months). Blood samples could not be drawn in 6 residents, therefore data is presented for 495 subjects (SUPPLEMENTAL FIGURE 1). Information regarding comorbidities and current prescribed medication were retrieved from medical records, physical examinations and questionnaires. The presence of heart failure was assessed by a panel of two cardiologists and a geriatrician, who were blinded for (hs-)cTn results. The diagnosis of heart failure was based on clinical examinations (including history, physical examinations, electrocardiography, echocardiography and if in doubt NTproBNP concentrations), patient records and questionnaires. A detailed study protocol has been published previously [18]. One year after inclusion, medical records at the nursing home were searched again for the occurrence of all-cause mortality. Follow-up data was retrieved from 466 residents (94\%) due to transfer to non-participating healthcare centres.

This study was conducted according to the principles of the Declaration of Helsinki and approved by the local ethics committee. Written informed consent was obtained from all subjects.

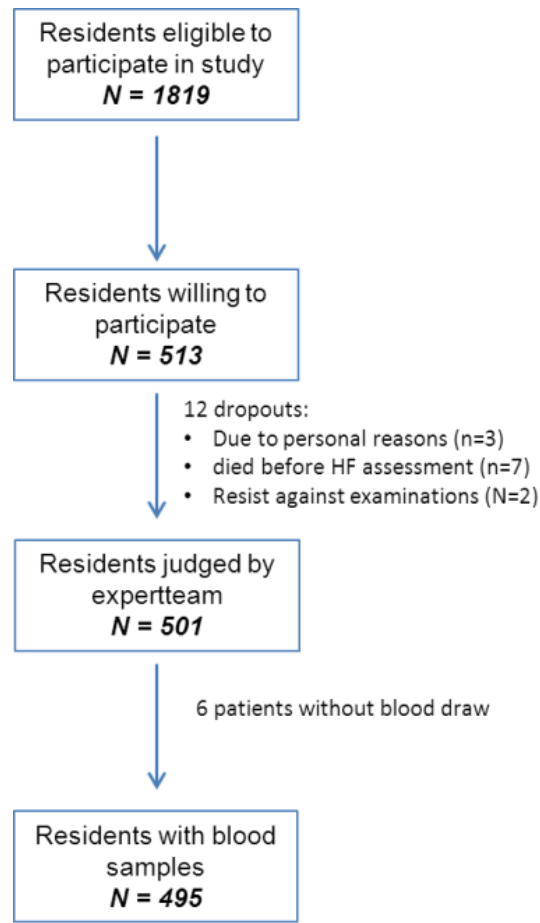

SUPPLEMENTAL FIGURE 1. Flow chart of included residents 


\section{Biochemical analysis}

Serum samples were collected, processed within 5 hours in aliquots and directly stored at $-80^{\circ} \mathrm{C}$ until analysis. Serum creatinine, cystatin C, NTproBNP and hscTnT concentrations were measured on the Cobas 6000 analyzer (Roche Diagnostics, Mannheim, Germany). Creatinine concentrations were assessed using the enzymatic method (Roche Diagnostics). Cystatin $\mathrm{C}$ was measured using a particle-enhanced turbidimetric assay (Gentian AS, Moss, Norway), that was standardized against the certified ERM-DA471/IFCC cystatin C reference material [19]. The glomerular filtration rate was estimated by the Chronic Kidney Disease Epidemiology Collaboration [20] and the Berlin Initiative Study (BIS)-2 equation [21], both using serum creatinine and cystatin $C$ concentrations. HscTnT concentrations were determined using the high-sensitivity cTnT assay (Roche Diagnostics; lotnumber 167650), with a $99^{\text {th }}$ percentile upper reference limit of $14 \mathrm{ng} / \mathrm{L}$ and a $10 \%$ coefficient of variation (CV) cutoff at $13 \mathrm{ng} / \mathrm{L}$ and limit of detection (LoD) at $5 \mathrm{ng} / \mathrm{L}$. Gender-specific cutoffs were reported at $14.5 \mathrm{ng} / \mathrm{L}$ and $10 \mathrm{ng} / \mathrm{L}$ for males and females, respectively [22]. Hs-cTnl measurements were performed on the ARCHITECT i2000SR platform using the precommercial ARCHITECT STAT high-sensitivity troponin I (hs-cTnl) assay (Abbott Laboratories, Hoofddorp, the Netherlands). According to the manufacturer, LoD and $10 \% \mathrm{CV}$ cutoff were reached at $1.1-1.9$ and $4.7 \mathrm{ng} / \mathrm{L}$, respectively and the $99^{\text {th }}$ percentile cut-off concentration for the overall population was at $26.2 \mathrm{ng} / \mathrm{L}$. Gender-specific cut-offs at $34.2 \mathrm{ng} / \mathrm{L}$ and $15.6 \mathrm{ng} / \mathrm{L}$ were also defined for males and females, respectively. Also, cTnl was measured using the guideline acceptable Access-3 Tnl assay (Beckman Coulter) on the DXL platform. The $99^{\text {th }}$ percentile and $10 \% \mathrm{CV}$ cut-off concentration were both established at 40 $\mathrm{ng} / \mathrm{L}$ by the manufacturer. All biochemical analysis were performed in freshly thawed aliquots, except for Beckman cTnl results that were measured after one additional freeze-thaw cycle.

\section{Echocardiography analysis}

Echocardiography was performed by an expert echocardiographist, who was blinded for hs-cTn concentrations. Transthoracic images of the left ventricle (LV) were acquired to assess morphology, function and mass (Philips CX-50, Philips 
Medical Systems, Best, the Netherlands). LV function and -mass were calculated by off-line image analysis using Xcelera software package (Philips Medical Systems, Best, the Netherlands), according to current ESC/AHA guidelines [23]. Left ventricular hypertrophy (LVH) was defined as an LVmass $>95 \mathrm{~g} / \mathrm{m}^{2}$ in females or LVmass $>115 \mathrm{~g} / \mathrm{m}^{2}$ in males. Echocardiographic analysis could not be performed in 51 residents due to impaired image quality.

\section{Selection criteria of healthier subpopulations}

From the complete nursing home cohort $(n=495)$, increasingly healthier subjects were selected using gradually more rigorous selection criteria. In Subgroup 1 $(\mathrm{n}=322 ; 65 \%)$ all residents with heart failure, diagnosed during this study were excluded from the original cohort. In subgroup $2(n=196 ; 40 \%)$ all residents with heart failure, LVH, LVEF $<55 \%$ and NTproBNP concentrations $>400 \mathrm{pg} / \mathrm{mL}$ (47 pmol/L) were excluded. Subgroup $3(n=154 ; 31 \%)$ encompassed the residents of subgroup 2, except for those with a history of cardiovascular events, defined as acute myocardial infarction, coronary artery disease, cardiac surgery or revascularisation procedures performed and prior stroke. Finally, subgroup 4 $(n=97 ; 20 \%$ ) consisted of the residents of subgroup 3 , excluding those with known impaired kidney functions or an eGFR $<60 \mathrm{~mL} / \mathrm{min} / 1.73 \mathrm{~m}^{2}$.

\section{Statistical analysis}

Comparisons of baseline characteristics were performed using the T-test for continuous variables with a normal distribution, Mann-Whitney U-test for nonnormal distributed continuous variables and Chi square test for categorical variables. Data are presented as proportions, means \pm standard deviations (SD), and data with a non-normal distribution are given as the median (interquartile range, IQR). Due to the low amount of $(<300)$ residents in the increasingly healthier subpopulations, $97.5^{\text {th }}$ and $95^{\text {th }}$ percentile (hs-)cTn cutoffs were computed instead of the $99^{\text {th }}$ percentile URL. The $95 \%$ confidence intervals $(\mathrm{Cl})$ of the $97.5^{\text {th }}$ and $95^{\text {th }}$ percentile cut-offs were calculated using 10.000 bootstrap samples. The relative contribution of renal function and heart failure to (hs-)cTn concentrations was assessed by logistic regression analyses with hs-cTnT, hs-cTnl or cTnl in the $4^{\text {th }}$ quartile as the dependent categorical variable. Furthermore, linear regression analysis and Pearson $\mathrm{R}$ correlations 
were performed with the natural logarithm (Ln) of hs-cTnT and hs-cTnl as dependent categorical variable, to normalize their skewed distribution. The prognostic value of (hs-)cTn concentrations was investigated by univariable and multivariable Cox-proportional hazards models. Kaplan Meier plots were constructed to illustrate survival rates.

Statistical analysis was performed with SPSS 20.0 (SPSS Inc, Chicago, IL, USA). Two sided $p$-values of $<0.05$ were considered statistically significant.

\section{Results}

\section{Baseline characteristics and the association to (hs-)cTn concentrations}

In this study, participants were on average $82 \pm 7$ years old and $64 \%$ was female. Of all residents with heart failure (34\%), $48 \%$ of the residents suffered from heart failure with a reduced ejection fraction (LVEF $<50 \%)$. An overview of other baseline characteristics is presented in TABLE 1.

The distribution of baseline hs-cTnT and hs-cTnl concentrations is illustrated in FIGURE 1. Median (IQR) concentrations were 20.6 (17.8-30.6) ng/L, 6.8 (4.112.5) $\mathrm{ng} / \mathrm{L}$ and $4.0(2.0-8.0) \mathrm{ng} / \mathrm{L}$ for hs-cTnT, hs-cTnl and cTnl respectively (TABLE 1). The results of the two cTnl assays were highly correlated (Pearson $\mathrm{R}(95 \% \mathrm{Cl}): 0.884$ (0.860-0.904), $\mathrm{p}<0.001)$, whereas associations between hscTnT assay and either hs-cTnl (Pearson R (95\%Cl):0.580 (0.515-0.638); $\mathrm{p}<0.001)$ or the cTnl-assay (Pearson R $(95 \% \mathrm{Cl}): 0.548(0.483-0.608) ; p<0.001)$ were significantly weaker. In total, $79 \%$ had elevated hs-cTnT concentrations $\left(>99^{\text {th }}\right.$ percentile), while only $9 \%$ and $5 \%$ of hs-cTnl and cTnl concentrations were elevated. When applying gender-specific cut-offs $89 \%$ and $16 \%$, respectively, had elevated hs-cTnT and hs-cTnl concentrations. 
TABLE 1. Baseline characteristics of a cohort of aged nursing home residents. *, indicates more than $10 \%$ missing data.

\begin{tabular}{|c|c|}
\hline & $\begin{array}{l}\text { All residents } \\
(n=495)\end{array}$ \\
\hline \multicolumn{2}{|l|}{ Traditional cardiovascular risk factors } \\
\hline Age (years), mean $( \pm S D)$ & $82( \pm 7)$ \\
\hline Male gender, $\%$ & $36 \%$ \\
\hline Body mass index, mean $( \pm S D)$ & $25.1( \pm 5.2)$ \\
\hline Hypertension, \% & $53 \%$ \\
\hline Diabetes mellitus, $\%$ & $22 \%$ \\
\hline Hypercholesterolemia, $\%{ }^{1}$ & $29 \%^{1}$ \\
\hline smokers, $\%$ & $14 \%$ \\
\hline \multicolumn{2}{|l|}{ History, $\mathrm{n}(\%)$} \\
\hline Prior cardiac event (total) & $33 \%$ \\
\hline Prior CAD (angina) & $23 \%$ \\
\hline Prior myocardial infarction & $16 \%$ \\
\hline Prior cardiac surgery (pacemaker, heart valve) & $5 \%$ \\
\hline Prior revascularization (CABG, $\mathrm{PCI})$ & $10 \%$ \\
\hline Prior stroke (CVA) & $41 \%$ \\
\hline \multicolumn{2}{|l|}{ Comorbidities, $\%$} \\
\hline Heart failure ${ }^{2}$ & $34 \%$ \\
\hline Impaired kidney function & $12 \%$ \\
\hline COPD & $17 \%$ \\
\hline Dementia & $57 \%$ \\
\hline \multicolumn{2}{|l|}{ Medication use } \\
\hline Statins & $26 \%$ \\
\hline Beta-blokkers & $30 \%$ \\
\hline Digoxin & $8 \%$ \\
\hline Diuretics & $39 \%$ \\
\hline ACE inhibitors & $18 \%$ \\
\hline Nitrates & $17 \%$ \\
\hline Ca-antagonists & $12 \%$ \\
\hline \multicolumn{2}{|l|}{ Echocardiography ${ }^{2}$} \\
\hline LVmass $(\mathrm{g})$, mean $( \pm S D)$ & $137.2( \pm 51.8)$ \\
\hline LVMI $\left(\mathrm{g} / \mathrm{m}^{2}\right)$, mean $( \pm S D)$ & $78.5( \pm 26.7)$ \\
\hline $\operatorname{LVEF}(\%)$, mean $( \pm S D)$ & $55.3( \pm 11.8)$ \\
\hline $\mathrm{LVH}, \%$ & $14 \%^{2}$ \\
\hline \multicolumn{2}{|l|}{ Biochemistry } \\
\hline NTproBNP (pmol/L), median (IQR) & $48(20-117)$ \\
\hline Creatinine $(\mu \mathrm{mol} / \mathrm{L})$, mean $( \pm \mathrm{SD})$ & $89.5( \pm 52.1)$ \\
\hline Cystatin C (mg/L), mean $( \pm S D)$ & $1.3( \pm 0.53)$ \\
\hline eGFRcKD EPI (creat-cys c) $\left(\mathrm{mL} / \mathrm{min} / 1.73 \mathrm{~m}^{2}\right)$, mean $( \pm \mathrm{SD})$ & $58.8( \pm 20.1)$ \\
\hline eGFRBIS-2 $\left(\mathrm{mL} / \mathrm{min} / 1.73 \mathrm{~m}^{2}\right)$, mean $( \pm \mathrm{SD})$ & $53.2( \pm 16.3)$ \\
\hline Roche hs-cTnT (ng/L), median (IQR) & $20.6(17.8-30.6)$ \\
\hline Abbott hs-cTnl (ng/L), median (IQR) & $6.8(4.1-12.5)$ \\
\hline Beckman cTnl (ng/L), median (IQR) & $4.0(2.0-8.0)$ \\
\hline
\end{tabular}



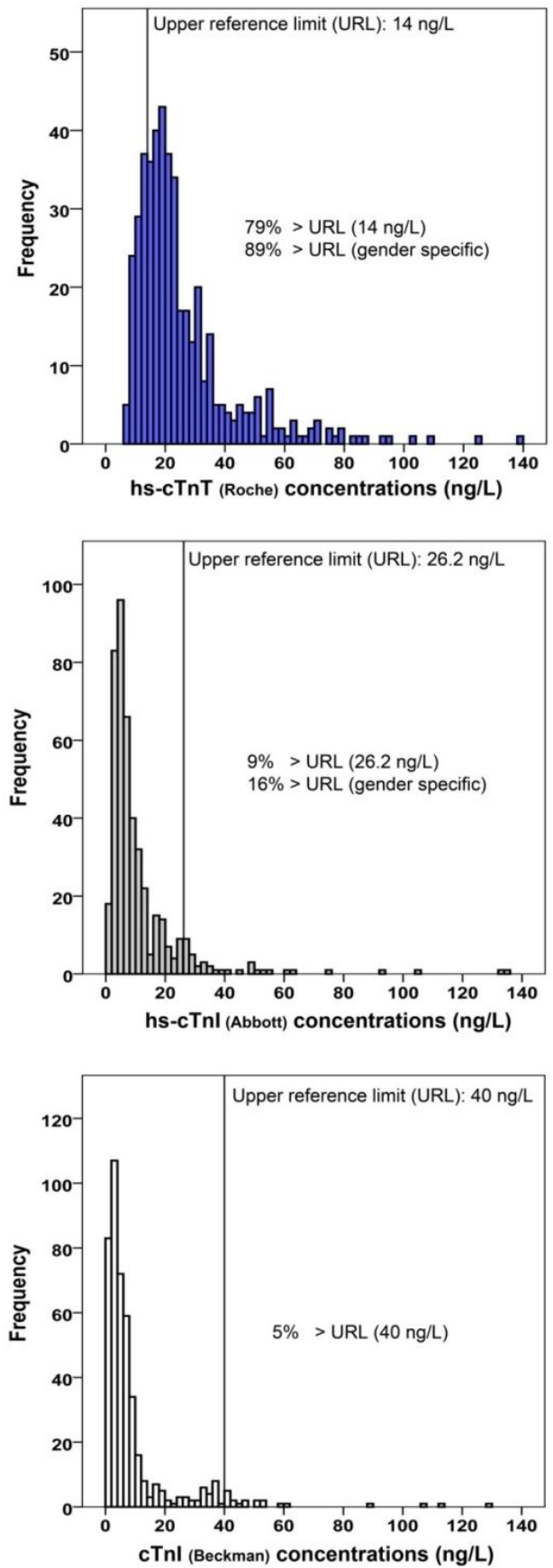

FIGURE 1. Distribution of hs-cTnT $T_{\text {Roche, }}$ hs-cTnl $\mathrm{Abbott}_{\text {and }} \mathrm{cTnl}_{\text {Beckman }}$ concentrations in aged nursing home residents. 
TABLE 2. Median (IQR) hs-cTnT, hs-cTnl and cTnl concentrations in fragile elderly according to the presence or absence of traditional risk factors as well as heart and kidney dysfunction.

\begin{tabular}{|c|c|c|c|c|c|c|c|c|c|}
\hline \multirow[b]{2}{*}{ Determinant } & \multicolumn{2}{|c|}{$\begin{array}{l}\text { Median (IQR) hs-cTnT } \\
\text { (Roche) } \\
\text { concentrations (ng/L), } \\
\text { when determinant is: }\end{array}$} & \multirow[t]{2}{*}{$\begin{array}{l}\mathrm{P} \text { - } \\
\text { value }\end{array}$} & \multicolumn{2}{|c|}{$\begin{array}{c}\text { Median (IQR) hs-cTnl } \\
\text { (Abbott) } \\
\text { concentrations (ng/L), } \\
\text { when determinant is: }\end{array}$} & \multirow[t]{2}{*}{$\begin{array}{c}\mathrm{P} \text { - } \\
\text { value }\end{array}$} & \multicolumn{2}{|c|}{$\begin{array}{c}\text { Median (IQR) cTnl } \\
\text { (Beckman) } \\
\text { concentrations (ng/L), } \\
\text { when determinant is: }\end{array}$} & \multirow[t]{2}{*}{$\begin{array}{c}\mathrm{P}- \\
\text { value }\end{array}$} \\
\hline & not present & present & & $\begin{array}{c}\text { not } \\
\text { present }\end{array}$ & present & & $\begin{array}{c}\text { not } \\
\text { present }\end{array}$ & present & \\
\hline Male sex & $\begin{array}{c}19.7 \\
(14.2-28.4)\end{array}$ & $\begin{array}{c}21.8 \\
(16.0-35.4)\end{array}$ & 0.014 & $\begin{array}{c}6.5 \\
(4.1-12.6)\end{array}$ & $\begin{array}{c}7.2 \\
(4.4-12.3)\end{array}$ & 0.337 & $\begin{array}{c}4.0 \\
(2.0-9.0)\end{array}$ & $\begin{array}{c}4.0 \\
(2.0-7.0)\end{array}$ & 0.756 \\
\hline Hypertension & $\begin{array}{c}19.0 \\
(13.3-27.8)\end{array}$ & $\begin{array}{c}21.8 \\
(16.0-35.1)\end{array}$ & $<0.001$ & $\begin{array}{c}5.9 \\
(3.8-10.4)\end{array}$ & $\begin{array}{c}7.2 \\
(4.4-13.5)\end{array}$ & 0.015 & $\begin{array}{c}4.0 \\
(2.0-7.0)\end{array}$ & $\begin{array}{c}4.0 \\
(2.0-9.0)\end{array}$ & 0.212 \\
\hline Diabetes & $\begin{array}{c}20.0 \\
(14.5-30.0)\end{array}$ & $\begin{array}{c}22.2 \\
(15.7-36.2)\end{array}$ & 0.044 & $\begin{array}{c}6.8 \\
(4.1-12.4)\end{array}$ & $\begin{array}{c}6.6 \\
(4.2-13.1)\end{array}$ & 0.930 & $\begin{array}{c}4.0 \\
(2.0-8.0)\end{array}$ & $\begin{array}{c}4.5 \\
(2.0-9.0)\end{array}$ & 0.529 \\
\hline Hypercholesterolemia & $\begin{array}{c}20.2 \\
(14.5-31.3)\end{array}$ & $\begin{array}{c}21.1 \\
(15.5-28.8)\end{array}$ & 0.899 & $\begin{array}{c}7.1 \\
(4.1-12.7)\end{array}$ & $\begin{array}{c}6.3 \\
(4.4-11.1)\end{array}$ & 0.264 & $\begin{array}{c}4.0 \\
(2.0-8.0)\end{array}$ & $\begin{array}{c}4.0 \\
(2.0-7.5)\end{array}$ & 0.374 \\
\hline Smoking & $\begin{array}{c}21.0 \\
(15.0-30.7)\end{array}$ & $\begin{array}{c}18.4 \\
(12.6-30.5)\end{array}$ & 0.227 & $\begin{array}{c}6.8 \\
(4.2-12.4)\end{array}$ & $\begin{array}{c}6.6 \\
(3.7-12.0)\end{array}$ & 0.997 & $\begin{array}{c}4.0 \\
(2.0-8.0)\end{array}$ & $\begin{array}{c}3.0 \\
(2.0-7.0)\end{array}$ & 0.811 \\
\hline $\begin{array}{l}\text { Prior cardiac event } \\
\text { (total) }\end{array}$ & $\begin{array}{c}19.3 \\
(14.1-28.3)\end{array}$ & $\begin{array}{c}23.4 \\
(16.5-35.9)\end{array}$ & $<0.001$ & $\begin{array}{c}5.9 \\
(3.8-10.5)\end{array}$ & $\begin{array}{c}8.3 \\
(5.2-18.4)\end{array}$ & $<0.001$ & $\begin{array}{c}3.0 \\
(2.0-7.0)\end{array}$ & $\begin{array}{c}6.0 \\
(3.0-12.0)\end{array}$ & $<0.001$ \\
\hline Prior MI & $\begin{array}{c}19.8 \\
(14.5-29.4)\end{array}$ & $\begin{array}{c}23.6 \\
(18.2-45.1)\end{array}$ & 0.001 & $\begin{array}{c}6.3 \\
(4.0-11.4)\end{array}$ & $\begin{array}{c}8.2 \\
(5.8-19.2)\end{array}$ & $<0.001$ & $\begin{array}{c}4.0 \\
(2.0-7.0)\end{array}$ & $\begin{array}{c}6.0 \\
(3.0-12.0)\end{array}$ & 0.001 \\
\hline Prior stroke & $\begin{array}{c}20.0 \\
(14.6-31.0)\end{array}$ & $\begin{array}{c}21.6 \\
(15.5-29.0)\end{array}$ & 0.475 & $\begin{array}{c}6.4 \\
(4.1-11.3)\end{array}$ & $\begin{array}{c}7.3 \\
(4.1-13.7)\end{array}$ & 0.501 & $\begin{array}{c}4.0 \\
(2.0-8.0)\end{array}$ & $\begin{array}{c}5.0 \\
(2.0-8.0)\end{array}$ & 0.174 \\
\hline COPD & $\begin{array}{c}20.0 \\
(14.8-28.8)\end{array}$ & $\begin{array}{c}23.5 \\
(14.9- \\
3538)\end{array}$ & 0.033 & $\begin{array}{c}6.4 \\
(4.1-11.3)\end{array}$ & $\begin{array}{c}10.0 \\
(5.0-17.5)\end{array}$ & 0.017 & $\begin{array}{c}4.0 \\
(2.0-8.0)\end{array}$ & $\begin{array}{c}7.0 \\
(2.0-10.0)\end{array}$ & 0.004 \\
\hline Dementia & $\begin{array}{c}21.8 \\
(16.4-33.0)\end{array}$ & $\begin{array}{c}19.5 \\
(14.3-28.3)\end{array}$ & 0.008 & $\begin{array}{c}7.7 \\
(4.3-13.4)\end{array}$ & $\begin{array}{c}6.4 \\
(4.0-11.3)\end{array}$ & 0.335 & $\begin{array}{c}5.0 \\
(2.0-8.0)\end{array}$ & $\begin{array}{c}4.0 \\
(2.0-7.0)\end{array}$ & 0.020 \\
\hline Heart failure & $\begin{array}{c}18.6 \\
(13.2-25.4)\end{array}$ & $\begin{array}{c}26.4 \\
(18.7-43.8)\end{array}$ & $<0.001$ & $\begin{array}{c}5.1 \\
(3.6-8.5)\end{array}$ & $\begin{array}{c}11.4 \\
(7.2-20.3)\end{array}$ & $<0.001$ & $\begin{array}{c}3.0 \\
(1.0-6.0)\end{array}$ & $\begin{array}{c}7.0 \\
(4.0-16.0)\end{array}$ & $<0.001$ \\
\hline$H F$ reduced $L V E F$ & $\begin{array}{c}24.0 \\
(17.4-37.2)\end{array}$ & $\begin{array}{c}25.6 \\
(18.6-35.9)\end{array}$ & 0.751 & $\begin{array}{c}11.1 \\
(7.0-18.9)\end{array}$ & $\begin{array}{c}11.6 \\
(6.6-19.1)\end{array}$ & 0.947 & $\begin{array}{c}7 \\
(4.0-16.5)\end{array}$ & $\begin{array}{c}7 \\
(4.0-16.8)\end{array}$ & 0.800 \\
\hline$L V H$ & $\begin{array}{c}20.1 \\
(14.5-28.6)\end{array}$ & $\begin{array}{c}21.5 \\
(16.2-31.0)\end{array}$ & 0.609 & $\begin{array}{c}6.4 \\
(3.8-11.4)\end{array}$ & $\begin{array}{c}5.9 \\
(4.1-10.7)\end{array}$ & 0.010 & $\begin{array}{c}4.0 \\
(2.0-7.0)\end{array}$ & $\begin{array}{c}7.0 \\
(2.0-12.0)\end{array}$ & 0.033 \\
\hline$L V E F<40 \%$ & $\begin{array}{c}19.6 \\
(14.3-28.2)\end{array}$ & $\begin{array}{c}25.6 \\
(18.3-35.4)\end{array}$ & 0.022 & $\begin{array}{c}6.4 \\
(3.8-11.0)\end{array}$ & $\begin{array}{c}11.4 \\
(5.7-22.3)\end{array}$ & 0.008 & $\begin{array}{c}4.0 \\
(2.0-7.0)\end{array}$ & $\begin{array}{c}7.0 \\
(4.0-19.0)\end{array}$ & 0.004 \\
\hline$L V E F<50 \%$ & $\begin{array}{c}19.3 \\
(14.1-27.2)\end{array}$ & $\begin{array}{c}22.5 \\
(17.6-33.2)\end{array}$ & 0.017 & $\begin{array}{c}6.3 \\
(3.7-10.4)\end{array}$ & $\begin{array}{c}9.6 \\
(5.1-18.4)\end{array}$ & 0.005 & $\begin{array}{c}4.0 \\
(2.0-6.0)\end{array}$ & $\begin{array}{c}7.0 \\
(3.0-11.0)\end{array}$ & 0.002 \\
\hline $\begin{array}{l}\text { Impaired kidney } \\
\text { function }\end{array}$ & $\begin{array}{c}19.9 \\
(14.5-28.5)\end{array}$ & $\begin{array}{c}28.9 \\
(19.4-54.9)\end{array}$ & $<0.001$ & $\begin{array}{c}6.5 \\
(4.1-11.4)\end{array}$ & $\begin{array}{c}7.8 \\
(4.8-20.6)\end{array}$ & 0.013 & $\begin{array}{c}4.0 \\
(2.0-8.0)\end{array}$ & $\begin{array}{c}6.0 \\
(3.0-26.0)\end{array}$ & 0.397 \\
\hline $\begin{array}{l}\text { eGFR } \text { cysc }_{\text {ckre }} \\
\quad<60 \mathrm{~mL} / \mathrm{min}\end{array}$ & $\begin{array}{c}18.0 \\
(13.2-23.2)\end{array}$ & $\begin{array}{c}25.2 \\
(16.8-36.4)\end{array}$ & $<0.001$ & $\begin{array}{c}5.1 \\
(3.7-8.7)\end{array}$ & $\begin{array}{c}8.1 \\
(4.8-16.6)\end{array}$ & $<0.001$ & $\begin{array}{c}3.0 \\
(1.0-6.0)\end{array}$ & $\begin{array}{c}6.0 \\
(3.0-11.0)\end{array}$ & $<0.001$ \\
\hline $\begin{array}{l}e G F R_{B I S-2} \\
\quad<60 \mathrm{~mL} / \mathrm{min}\end{array}$ & $\begin{array}{c}18.1 \\
(13.3-23.3)\end{array}$ & $\begin{array}{c}25.2 \\
(16.8-36.5)\end{array}$ & $<0.001$ & $\begin{array}{c}5.6 \\
(4.2-9.9)\end{array}$ & $\begin{array}{c}12.4 \\
(7.5-25.5)\end{array}$ & $<0.001$ & $\begin{array}{c}3.0 \\
(1.0-5.0)\end{array}$ & $\begin{array}{c}5.0 \\
(2.0-10.0)\end{array}$ & $<0.001$ \\
\hline Statins & $\begin{array}{c}21.2 \\
(14.6-31.2\end{array}$ & $\begin{array}{c}19.8 \\
(15.2-28.6)\end{array}$ & 0.858 & $\begin{array}{c}6.8 \\
(4.0-12.7)\end{array}$ & $\begin{array}{c}6.8 \\
(4.5-12.2)\end{array}$ & 0.890 & $\begin{array}{c}4.0 \\
(2.0-9.0)\end{array}$ & $\begin{array}{c}4.0 \\
(2.0-7.0)\end{array}$ & 0.559 \\
\hline Beta-blokkers & $\begin{array}{c}20.1 \\
(14.5-30.1)\end{array}$ & $\begin{array}{c}22.3 \\
(16.4-32.6)\end{array}$ & 0.036 & $\begin{array}{c}6.4 \\
(3.9-11.3)\end{array}$ & $\begin{array}{c}7.9 \\
(4.7-16.4)\end{array}$ & 0.009 & $\begin{array}{c}4.0 \\
(2.0-8.0)\end{array}$ & $\begin{array}{c}5.0 \\
(3.0-10.8)\end{array}$ & 0.017 \\
\hline Digoxin & $\begin{array}{c}20.3 \\
(14.7-30.0)\end{array}$ & $\begin{array}{c}22.6 \\
(16.0-41.5)\end{array}$ & 0.096 & $\begin{array}{c}6.4 \\
(4.1 \mathrm{v} 11.4)\end{array}$ & $\begin{array}{c}14.9 \\
(7.0-22.6)\end{array}$ & $<0.001$ & $\begin{array}{c}4.0 \\
(2.0-8.0)\end{array}$ & $\begin{array}{c}10.0 \\
(5.8-33.8)\end{array}$ & $<0.001$ \\
\hline Diuretics & $\begin{array}{c}18.8 \\
(13.3-25.4)\end{array}$ & $\begin{array}{c}25.3 \\
(17.7-39.6)\end{array}$ & $<0.001$ & $\begin{array}{c}5.7 \\
(3.8-9.6)\end{array}$ & $\begin{array}{c}9.1 \\
(5.3-18.1)\end{array}$ & $<0.001$ & $\begin{array}{c}3.0 \\
(2.0-7.0)\end{array}$ & $\begin{array}{c}6.0 \\
(3.0-12.0)\end{array}$ & $<0.001$ \\
\hline ACE inhibitors & $\begin{array}{c}20.0 \\
(14.3-31.0)\end{array}$ & $\begin{array}{c}22.8 \\
(17.6-30.2)\end{array}$ & 0.033 & $\begin{array}{c}6.3 \\
(3.9-11.9)\end{array}$ & $\begin{array}{c}8.4 \\
(5.7-14.0)\end{array}$ & $<0.001$ & $\begin{array}{c}4.0 \\
(2.0-8.0)\end{array}$ & $\begin{array}{c}6.0 \\
(3.0-9.5)\end{array}$ & 0.005 \\
\hline Nitrates & $\begin{array}{c}20.1 \\
(14.6-29.7)\end{array}$ & $\begin{array}{c}22.5 \\
(15.5-36.0)\end{array}$ & 0.079 & $\begin{array}{c}6.3 \\
(4.0-11.4)\end{array}$ & $\begin{array}{c}8.5 \\
(5.5-17.1)\end{array}$ & 0.001 & $\begin{array}{c}4.0 \\
(2.0-8.0)\end{array}$ & $\begin{array}{c}6.0 \\
(3.0-12.5)\end{array}$ & 0.002 \\
\hline Ca-antagonists & $\begin{array}{c}21.1 \\
(14.8-31.0)\end{array}$ & $\begin{array}{c}18.1 \\
(14.6-25.5)\end{array}$ & 0.240 & $\begin{array}{c}6.8 \\
(4.1-12.6)\end{array}$ & $\begin{array}{c}6.9 \\
(4.9-10.9)\end{array}$ & 0.969 & $\begin{array}{c}4.0 \\
(2.0-9.0)\end{array}$ & $\begin{array}{c}4.0 \\
(2.0-7.0)\end{array}$ & 0.713 \\
\hline
\end{tabular}


As depicted in TABLE 2, significantly higher concentrations for all cTn results $(p<0.001)$ were found in residents with a cardiac event in their medical history, a reduced kidney function and on diuretics. Also in residents with heart failure, higher (hs-)cTn concentrations were found although median (hs-)cTn concentrations were not statistically different in subjects with a reduced versus preserved ejection fraction (all $\mathrm{p}>0.05$ ). Strikingly, higher hs-cTnT but not (hs-) cTnl concentrations were found in males compared to females, although only minor differences were observed. In contrast, (hs-)cTnl but not hs-cTnT were significantly higher in participants with LVH.

(Hs-)cTn concentrations in subpopulations without cardiac, echocardiographic and renal abnormalities

In the total cohort, $97.5^{\text {th }}(95 \% \mathrm{Cl})$ percentile cutoffs were established at 83.9 (71.2-108.1), 58.4 (40.8-123.8) and 52.0 (41.1-88.9) ng/L for hs-cTnT, hs-cTnl and cTnl respectively (FIGURE 2). These cutoffs decreased when subjects with heart failure and other cardiovascular diseases (subgroup 1-3) were excluded. As such, in residents without structural heart disease and prior cardiovascular events (subgroup 3 ) the $97.5^{\text {th }}$ percentile reduced to $62.8(38.3-75.2), 25.7$ (16.6-45.6) and 19.7 (9.4-218.7) $\mathrm{ng} / \mathrm{L}$ for hs-cTnT, hs-cTnl and cTnl, respectively.

Additionally, excluding participants with renal dysfunction (subgroup 4) resulted in a further reduction in the $97.5^{\text {th }}$ percentile for hs-cTnT to 56.5 (33.1-78.4) $\mathrm{ng} / \mathrm{L}$, but did not affect hs-cTnl (25.9 ng/L) and cTnl (17.8 ng/L).

Interestingly, 97.5 percentile cutoffs in all subgroups were substantially above the reported $99^{\text {th }}$ percentile URL for hs-cTnT $(14 \mathrm{ng} / \mathrm{L})$, but not for both hs-cTnl (26.2 ng/L) and cTnl (40 ng/L), as depicted in FIGURE 2. Similar trends were seen for the $95^{\text {th }}$ percentile (hs-)cTn concentrations among the different subgroups (FIGURE 2). 

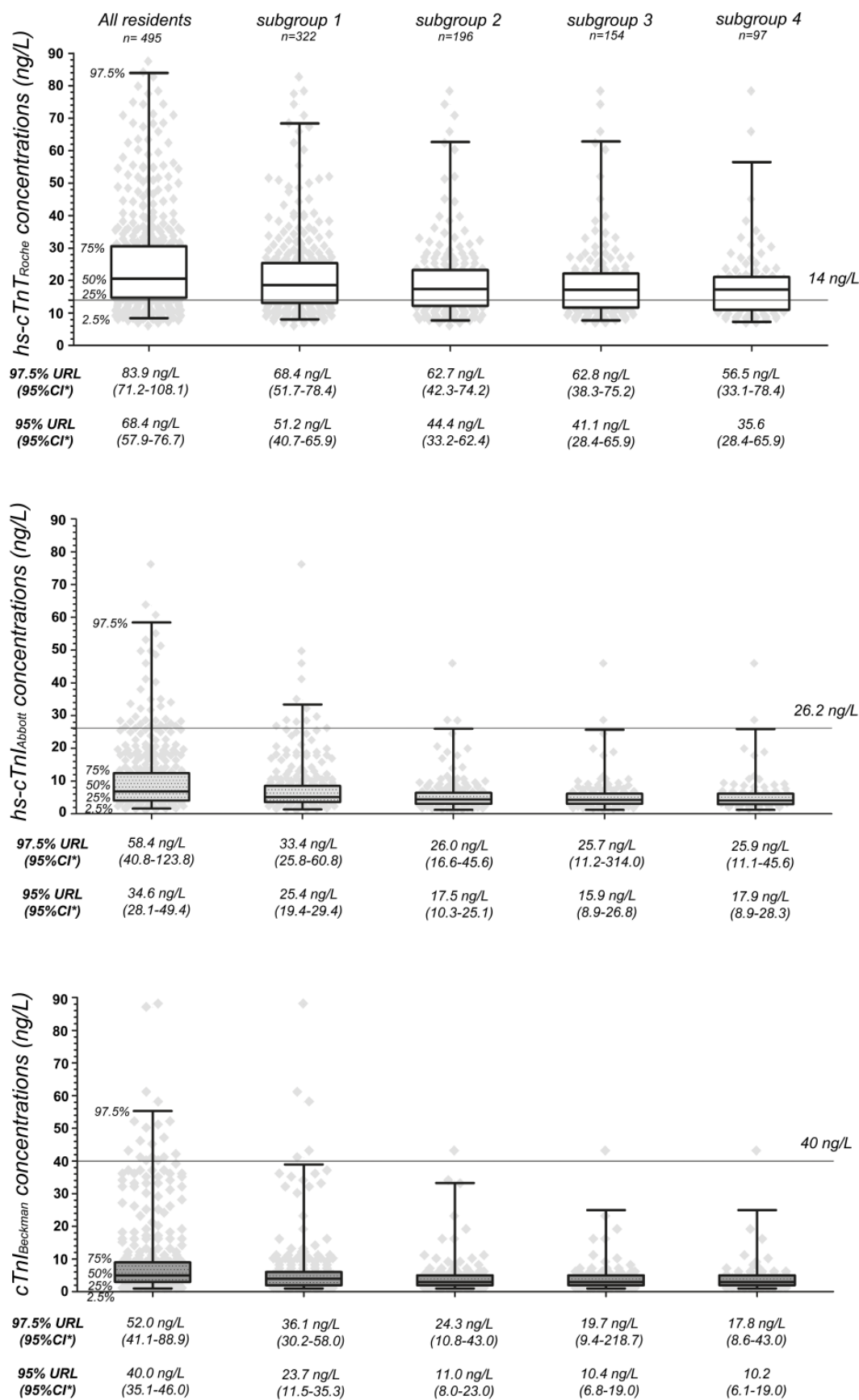

FIGURE 2. $97.5 \%$ and $95 \%$ upper reference limits ( $95 \%$ confidence intervals) of hs-cTnT, hs-cTnl and cTnl in increasingly cardio-and renal healthier subpopulations. ${ }^{*}$, indicates that $95 \% \mathrm{Cl}$ were constructed using 10.000 bootstrapping sample; Reported $99^{\text {th }}$ percentile URL for each cTn assay are indicated with horizontal lines. 


\section{Association of (hs-)cTn concentrations with heart failure and renal dysfunction}

Univariable logistic regression analysis demonstrated that the presence of heart failure increased the odds of having hs-cTnT, hs-cTnl and cTnl concentrations in the $4^{\text {th }}$ quartile by $4.3,4.9$ and 5.4 times, respectively (TABLE 3; all p<0.001). Furthermore, the presence of renal dysfunction (eGFR $<60 \mathrm{~mL} / \mathrm{min} / 1.73 \mathrm{~m}^{2}$ ) also accompanied with significantly higher (hs-)cTn concentrations, even beyond the presence of heart failure (TABLE 3; unadjusted multivariable model). Whereas both heart failure and decreases in eGFR were equal contributors to higher hscTnT concentrations (OR of respectively 3.4 and 3.6, $p<0.001$ ), associations of hs-cTnl and cTnl with renal dysfunction were less pronounced (OR of respectively, 1.9 and $2.1 ; p<0.01$ ) in comparison to heart failure (OR of respectively 4.3 and $4.7, \mathrm{p}<0.001$ ). The same results were found when linear regression analyses were performed (SUPPLEMENTAL TABLE 1-2).

TABLE 3. Logistic regression analysis to assess the association of heart failure and eGFR to higher hs-cTn concentrations

\begin{tabular}{|lll|lll}
\multicolumn{3}{|c|}{ univariable } & \multicolumn{3}{c}{ Unadjusted multivariable } \\
\hline OR & $95 \%(\mathrm{Cl})$ & p-value & OR & $95 \%(\mathrm{Cl})$ & p-value \\
\hline
\end{tabular}

\begin{tabular}{|c|c|c|c|c|c|c|}
\hline \multicolumn{7}{|c|}{ Outcome variable: Roche hs-cTnT in 4th quartile (>30.4 ng/L) } \\
\hline Heart failure & 4.3 & $(2.8-6.5)$ & $<0.001$ & 3.4 & $(2.2-5.4)$ & $<0.001$ \\
\hline eGFR & 4.5 & $(2.8-7.3)$ & $<0.001$ & 3.6 & $(2.2-5.9)$ & $<0.001$ \\
\hline \multicolumn{7}{|c|}{ Outcome variable: Abbott hs-cTnl in 4th quartile (>12.4 ng/L) } \\
\hline Heart failure & 4.9 & $(3.2-7.7)$ & $<0.001$ & 4.3 & $(2.7-6.8)$ & $<0.001$ \\
\hline eGFR & 2.6 & $(1.6-4.0)$ & $<0.001$ & 1.9 & $(1.2-3.1)$ & 0.007 \\
\hline \multicolumn{7}{|c|}{ Outcome variable: Beckman cTnl in 4th quartile (>8.0 ng/L) } \\
\hline Heart failure & 5.4 & $(3.4-8.4)$ & $<0.001$ & 4.7 & $(2.9-7.4)$ & $<0.001$ \\
\hline eGFR CKDEPI $_{1}<60 \mathrm{~mL} / \mathrm{min} / 1.73 \mathrm{~m}^{2}$ & 2.9 & $(1.8-4.6)$ & $<0.001$ & 2.1 & $(1.3-3.4)$ & 0.003 \\
\hline
\end{tabular}




\section{Prognostic value of hs-cTn concentrations for all-cause-mortality.}

During the follow-up period of one year, 109 subjects (23.4\%) died. These subjects were older, more frequently male and had a higher prevalence of heart failure and impaired kidney disease (SUPPLEMENTAL TABLE 3). Also, significantly higher hs-cTnT, hs-cTnl and cTnl concentrations were found in the residents that died. According to univariable Cox regression analysis, baseline hs-cTnT concentrations were a better predictor for all-cause mortality in comparison to both $\mathrm{cTnl}$ assays (FIGURE 3). Subjects with the highest hs-cTnT levels $\left(4^{\text {th }}\right.$ quartile) had a higher hazard ratio for all-cause mortality $(H R=1.8 ; p=0.004)$, compared to hs-cTnl (HR:1.3 $\mathrm{p}=0.300)$ and $\mathrm{cTnl}(\mathrm{HR}: 1.6$; $\mathrm{p}=0.041$ ) concentrations. Even when adjusting for other significant risk factors baseline hs-cTnT remained superior in the prediction for all-cause mortality (SUPPLEMENTAL TABLE 4).

FIGURE 3. Kaplan-meier curves for the estimation of risk on all-cause mortality using hs-cTnT, hs-cTnl and cTnl concentrations.

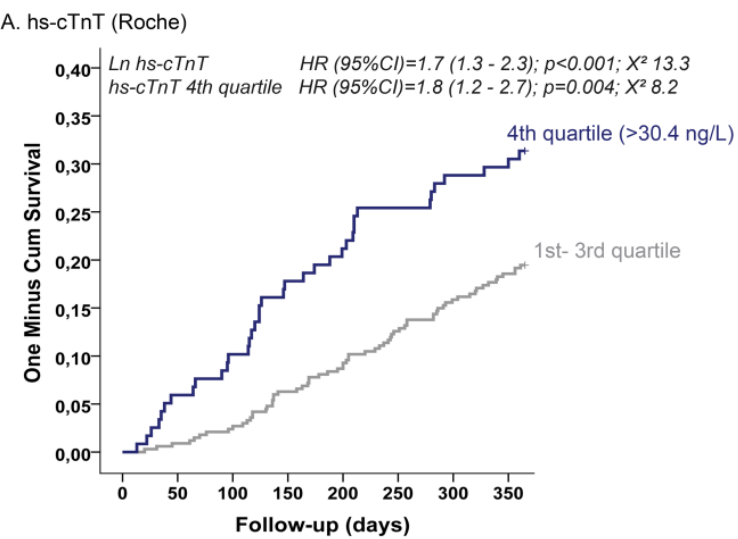

B. hs-cTnl (Abbott)

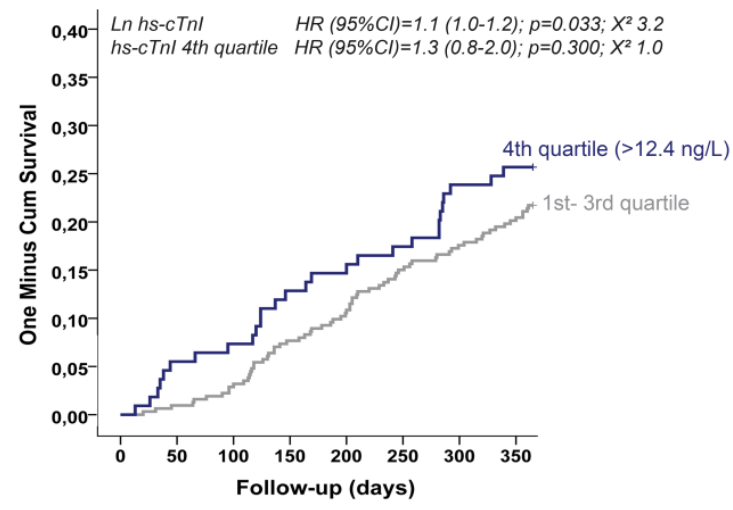

C. cTnl (Beckman)

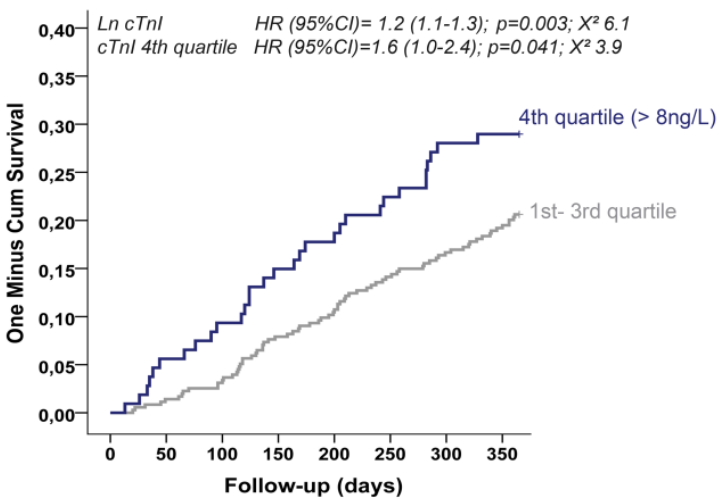




\section{Discussion}

In this multicentre study we found that hs-cTnT concentrations are above the stated normal range, even in those without any cardiovascular and renal disease. This however is not the case of hs-cTnl and cTnl results. Moreover, employing hs-cTnT assays in geriatric care appears to be associated not only by cardiovascular diseases but also renal dysfunction. Despite this, hs-cTnT remained the most important prognostic factor for all-cause mortality.

\section{Hs-cTnT is more affected by age than (hs-)cTnl}

In this nursing home cohort, median hs-cTnl and cTnl concentrations were much lower than hs-cTnT. Intriguingly, the reported $99^{\text {th }}$ percentile cutoffs for both (hs-)cTnl assays are set higher than for hs-cTnT, even when established in the same reference population [9, 15, 24]. Consequently, more positive results were observed for hs-cTnT than (hs-)cTnl elevations. Since, $99^{\text {th }}$ percentile URL of hs-cTn are reported to be highly affected by age, gender and selection criteria of the reference population [24, 25], more cardiac and renal healthy subgroups were selected to gain more insight into the distribution of hs-cTn in these elderly subjects. Even in these healthier subpopulations, $97.5^{\text {th }}$ percentile hs-cTnT values were still at least 4 times higher as compared to the healthy reference population (URL at $14 \mathrm{ng} / \mathrm{L}$ ), while around or below the reported URL for the hscTnl (26.2 ng/L) and cTnl (40 ng/L) assays. It has been described that higher hs-cTn cutoffs give a better diagnostic performance in the elderly $[5,10,11,15]$. This was especially true for hs-cTnT, with optimal cutoffs 4 to 5 fold higher as the established $99^{\text {th }}$ percentile URL in healthy reference populations $(14 \mathrm{ng} / \mathrm{L})$ $[12,26]$. Together with our results, these findings imply that in elderly (>65 years) a higher cutoff seems necessary for hs-cTnT, but might not be required for hs-cTnl.

\section{Hs-cTnT is more strongly associated with renal function than (hs-)cTnl}

The association with heart failure was equally as strong for all cTn assays. Remarkably, (hs-)cTnl appeared to be more associated with left ventricular function and morphology abnormalities than hs-cTnT in this cohort. However, both (hs-)cTnl and hs-cTnT results have been described to highly correlate with 
left ventricular measures in the elderly [3, 4]. Therefore, more detailed head-tohead comparisons between hs-cTnT and hs-cTnl assays should be performed to truly unravel these differences.

Furthermore, we found that hs-cTnT was more influenced by renal dysfunction than hs-cTnl. By excluding residents with renal dysfunction, a visible effect on the reduction in hs-cTnT URL was noticed, which is in line with previous observations in other study populations [24, 27], but was not found for (hs-)cTnl. In chronic kidney disease patients, a higher association of eGFR with hs-cTnT in comparison to hs-cTnl has also been reported [28]. By examining the contribution of age, heart failure and renal dysfunction to hs-cTn concentrations, we are the first to identify the magnitude of these determinants. As such, we found in frail nursing home residents that hs-cTnT concentrations were equally associated by the presence of heart failure and renal dysfunction, whereas the correlation of both (hs-)cTnl concentrations with renal dysfunction was only half as strong as with heart failure. We are the first to describe this using two different cTnl assays, suggesting biological differences between the cTnT and cTnl molecules rather than assay-related analytical influences. A plausible explanation for these findings could be a slower clearance of cTnT from the blood circulation by the kidneys in comparison to cTnl.

\section{Hs-cTnT is a better predictor for all-cause mortality than (hs-)cTnI}

A stronger association with all-cause mortality was found for hs-cTnT in comparison to both hs-cTnl and cTnl, suggesting a more prominent role for hscTnT in risk stratification. Notwithstanding, previous studies have clearly established the prognostic importance of hs-cTnl in the prediction for cardiovascular events and all-cause mortality [4, 12]. Our findings however do extend the results of previous work in acute populations where hs-cTnT outperformed (hs-)cTnl in its prognostic accuracy for the risk on all-cause mortality [27, 29, 30]. More recently, hs-cTnT appeared to have an additional prognostic importance independently from hs-cTnl [31], suggesting that hs-cTnT and hs-cTnl could contain different prognostic information. The rationale behind these findings could be the higher association of hs-cTnT with renal dysfunction. 


\section{Limitations}

Due to the small sample size, we were not able to compute the diagnostic cutoff at the $99^{\text {th }}$ percentile concentration of the more healthier subgroups.

Also, as the diagnosis of heart failure was assessed by an expert team, other clinical diagnoses were not. Echocardiographic analysis could however not be performed in 51 frail nursing home residents and subsequently the presence of heart failure could not be evaluated in these residents.

\section{Conclusion}

In summary, we challenge the application of the $99^{\text {th }}$ percentile cutoff in frail elderly (>65 years), especially for hs-cTnT. We found that hs-cTnT concentrations were elevated in almost all nursing home residents, in contrast to (hs-) cTnl concentrations, regardless from their cardiac and renal health. All hs-cTn assays were highly associated with heart failure and structural heart disease, while hs-cTnT was more associated with renal dysfunction than both (hs-)cTnl and had a more prominent role in assessing the risk on all-cause mortality. In daily practice, hs-cTnT and hs-cTnl results are used interchangeably to diagnose AMI. It however merits further research whether patients equally benefit from hs-cTnT and hs-cTnl measurements and as to whether such clinical benefit is dependent on age. 


\section{References}

1. Hancock, H.C., H. Close, J.M. Mason, et al., High prevalence of undetected heart failure in long-term care residents: findings from the Heart Failure in Care Homes (HFinCH) study. Eur J Heart Fail, 2013. 15(2): p. 158-65.

2. Messinger-Rapport, B.J., J.K. Gammack, M.O. Little, et al., Clinical update on nursing home medicine: 2014. J Am Med Dir Assoc, 2014. 15(11): p. 786-801.

3. deFilippi, C.R., J.A. de Lemos, R.H. Christenson, et al., Association of serial measures of cardiac troponin $T$ using a sensitive assay with incident heart failure and cardiovascular mortality in older adults. JAMA, 2010. 304(22): p. 2494-502.

4. Eggers, K.M., P. Venge, B. Lindahl, et al., Cardiac troponin I levels measured with a highsensitive assay increase over time and are strong predictors of mortality in an elderly population. J Am Coll Cardiol, 2013. 61(18): p. 1906-13.

5. Normann, J., M. Mueller, M. Biener, et al., Effect of older age on diagnostic and prognostic performance of high-sensitivity troponin $\mathrm{T}$ in patients presenting to an emergency department. Am Heart J, 2012. 164(5): p. 698-705 e4.

6. Masson, S., R. Latini, G.F. Mureddu, et al., High-sensitivity cardiac troponin T for detection of subtle abnormalities of cardiac phenotype in a general population of elderly individuals. J Intern Med, 2013. 273(3): p. 306-17.

7. Thygesen, K., J.S. Alpert, A.S. Jaffe, et al., Third universal definition of myocardial infarction. J Am Coll Cardiol, 2012. 60(16): p. 1581-98.

8. Apple, F.S., A.H. Wu, and A.S. Jaffe, European Society of Cardiology and American College of Cardiology guidelines for redefinition of myocardial infarction: how to use existing assays clinically and for clinical trials. Am Heart J, 2002. 144(6): p. 981-6.

9. Apple, F.S., R. Ler, and M.M. Murakami, Determination of 19 cardiac troponin I and T assay 99th percentile values from a common presumably healthy population. Clin Chem, 2012. 58(11): p. 1574-81.

10. Reiter, M., R. Twerenbold, T. Reichlin, et al., Early diagnosis of acute myocardial infarction in the elderly using more sensitive cardiac troponin assays. Eur Heart J, 2011. 32(11): p. 137989.

11. Olivieri, F., R. Galeazzi, D. Giavarina, et al., Aged-related increase of high sensitive Troponin $\mathrm{T}$ and its implication in acute myocardial infarction diagnosis of elderly patients. Mech Ageing Dev, 2012. 133(5): p. 300-5.

12. Eggers, K.M., L. Lind, P. Venge, et al., Factors influencing the 99th percentile of cardiac troponin I evaluated in community-dwelling individuals at 70 and 75 years of age. Clin Chem, 2013. 59(7): p. 1068-73.

13. Gore, M.O., S.L. Seliger, C.R. Defilippi, et al., Age- and sex-dependent upper reference limits for the high-sensitivity cardiac troponin T assay. J Am Coll Cardiol, 2014. 63(14): p. 1441-8.

14. Kuster, N., K. Monnier, G. Baptista, et al., Estimation of age- and comorbidities-adjusted percentiles of high-sensitivity cardiac troponin T levels in the elderly. Clin Chem Lab Med, 2014.

15. Menacer, S., Y.E. Claessens, C. Meune, et al., Reference range values of troponin measured by sensitive assays in elderly patients without any cardiac signs/symptoms. Clin Chim Acta, 2013. 417: p. 45-7.

16. Sandoval, Y. and F.S. Apple, The global need to define normality: the 99th percentile value of cardiac troponin. Clin Chem, 2014. 60(3): p. 455-62.

17. Jaffe, A.S. and F.S. Apple, High-sensitivity cardiac troponin assays: isn't it time for equality? Clin Chem, 2014. 60(1): p. 7-9.

18. Daamen, M.A., J.P. Hamers, A.P. Gorgels, et al., The prevalence and management of heart failure in Dutch nursing homes; design of a multi-centre cross-sectional study. BMC Geriatr, 2012. 12: p. 29.

19. Voskoboev, N.V., T.S. Larson, A.D. Rule, et al., Importance of cystatin C assay standardization. Clin Chem, 2011. 57(8): p. 1209-11. 
20. Inker, L.A., C.H. Schmid, H. Tighiouart, et al., Estimating glomerular filtration rate from serum creatinine and cystatin C. N Engl J Med, 2012. 367(1): p. 20-9.

21. Schaeffner, E.S., N. Ebert, P. Delanaye, et al., Two novel equations to estimate kidney function in persons aged 70 years or older. Ann Intern Med, 2012. 157(7): p. 471-81.

22. Giannitsis, E., K. Kurz, K. Hallermayer, et al., Analytical validation of a high-sensitivity cardiac troponin T assay. Clin Chem, 2010. 56(2): p. 254-61.

23. Lang, R.M., M. Bierig, R.B. Devereux, et al., Recommendations for chamber quantification. Eur J Echocardiogr, 2006. 7(2): p. 79-108.

24. Collinson, P.O., Y.M. Heung, D. Gaze, et al., Influence of population selection on the 99th percentile reference value for cardiac troponin assays. Clin Chem, 2012. 58(1): p. 219-25.

25. Cardinaels, E.P., A.M. Mingels, L.H. Jacobs, et al., A comprehensive review of upper reference limits reported for (high-)sensitivity cardiac troponin assays: the challenges that lie ahead. Clin Chem Lab Med, 2012. 50(5): p. 791-806.

26. McKie, P.M., D.M. Heublein, C.G. Scott, et al., Defining high-sensitivity cardiac troponin concentrations in the community. Clin Chem, 2013. 59(7): p. 1099-107.

27. Apple, F.S., M.M. Murakami, L.A. Pearce, et al., Predictive value of cardiac troponin I and T for subsequent death in end-stage renal disease. Circulation, 2002. 106(23): p. 2941-5.

28. deFilippi, C., S.L. Seliger, W. Kelley, et al., Interpreting cardiac troponin results from highsensitivity assays in chronic kidney disease without acute coronary syndrome. Clin Chem, 2012. 58(9): p. 1342-51.

29. Rubini Gimenez, M., R. Twerenbold, T. Reichlin, et al., Direct comparison of high-sensitivitycardiac troponin I vs. T for the early diagnosis of acute myocardial infarction. Eur Heart J, 2014. 35(34): p. 2303-11.

30. Haaf, P., T. Reichlin, R. Twerenbold, et al., Risk stratification in patients with acute chest pain using three high-sensitivity cardiac troponin assays. Eur Heart J, 2014. 35(6): p. 365-75.

31. Omland, T., M.A. Pfeffer, S.D. Solomon, et al., Prognostic value of cardiac troponin I measured with a highly sensitive assay in patients with stable coronary artery disease. J Am Coll Cardiol, 2013. 61(12): p. 1240-9. 


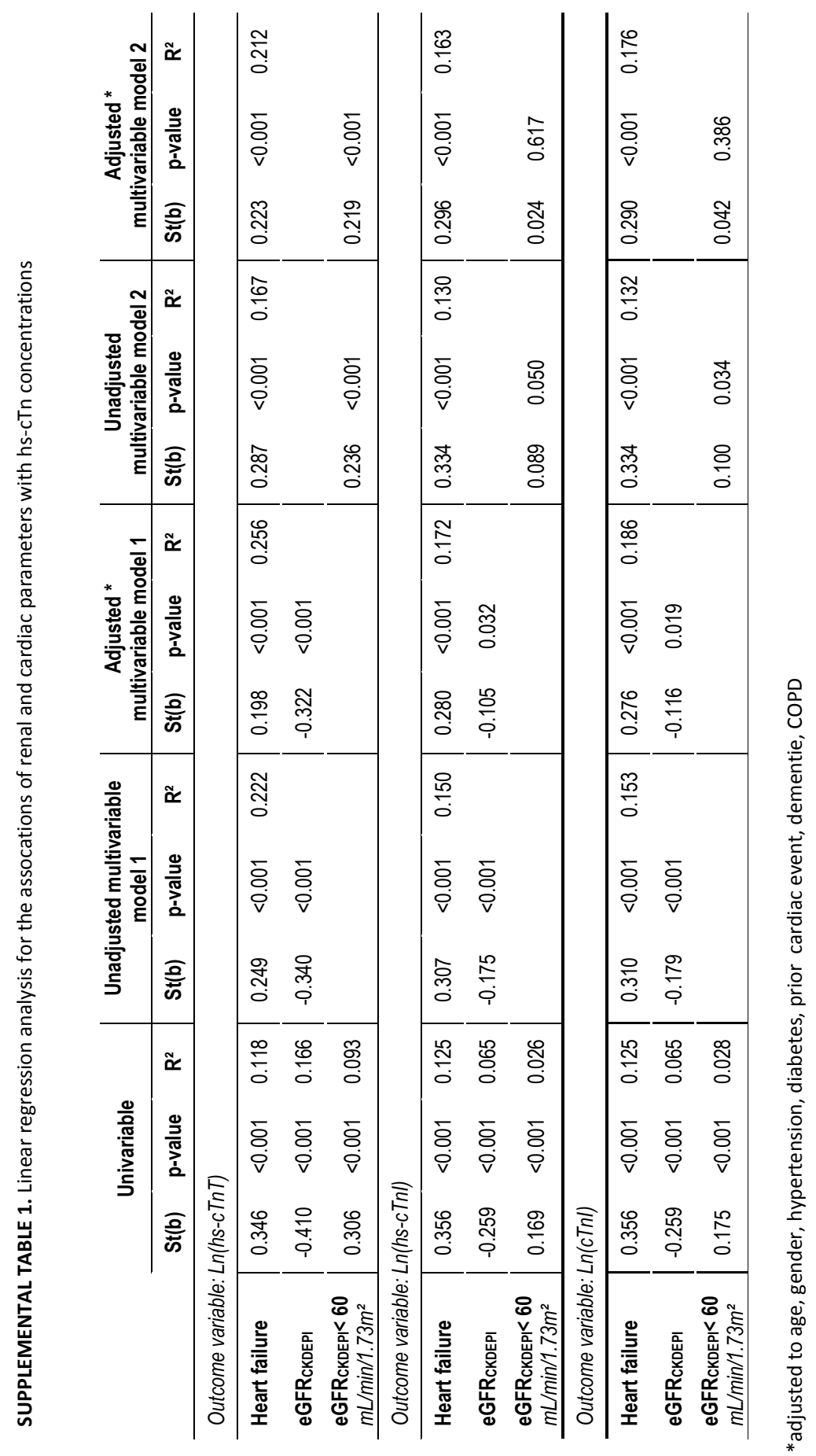




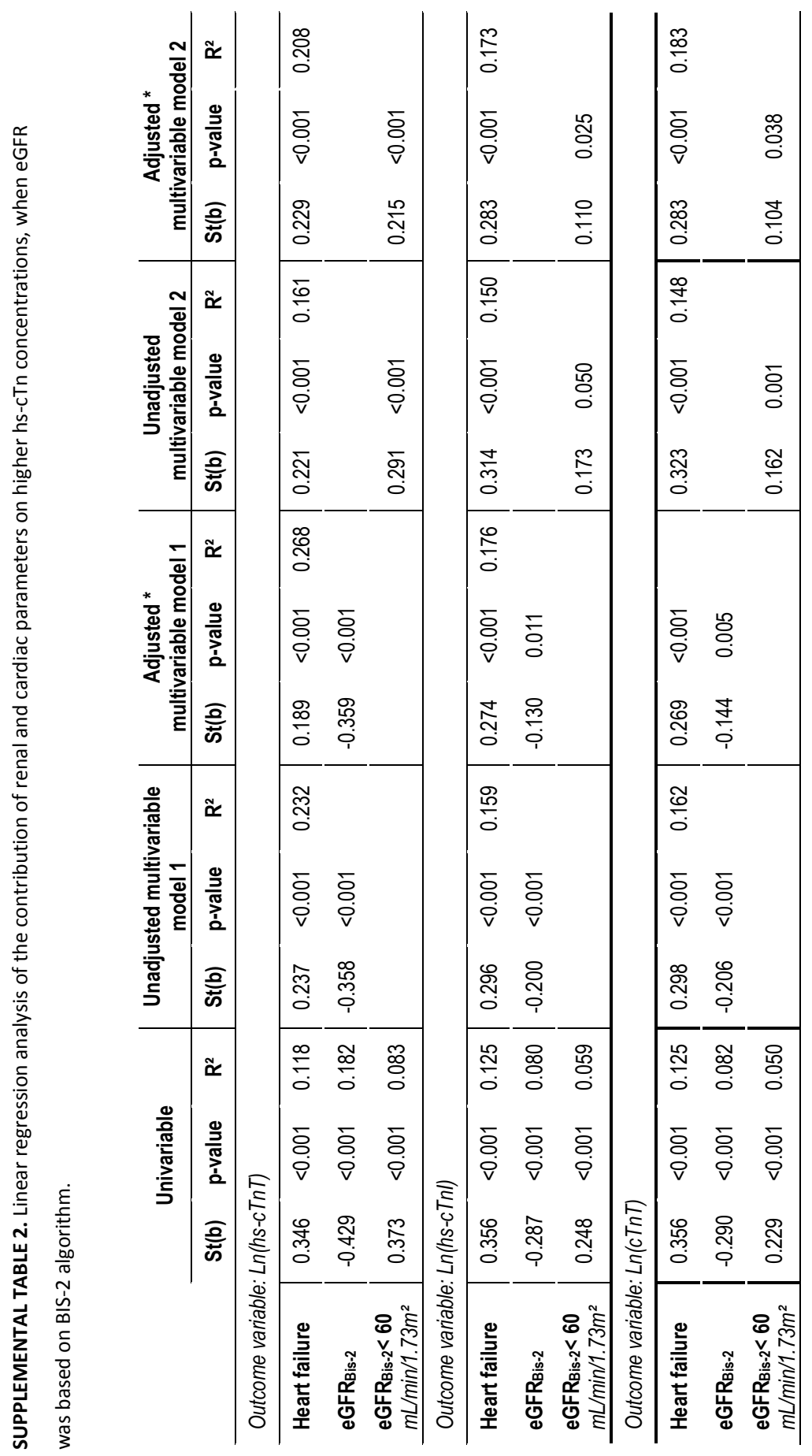


SUPPLEMENTAL TABLE 3. Associations of traditional risk factors, comorbidities, renal function and hs-cTn concentrations with the presence of all-cause mortality

\begin{tabular}{|c|c|c|c|}
\hline & \multicolumn{3}{|c|}{ All-cause-mortality } \\
\hline Determinant & Alive $(n=357)$ & Dead $(n=109)$ & p-value* \\
\hline \multicolumn{4}{|c|}{ Traditional cardiovascular risk factors } \\
\hline Age (years), mean $( \pm S D)$ & $81.9 \pm 6.8$ & $83.5 \pm 6.4$ & 0.035 \\
\hline Male gender, \% & $34 \%$ & $48 \%$ & 0.009 \\
\hline Body mass index, mean $( \pm S D)$ & $25.6 \pm 5.3$ & $24.2 \pm 5.3$ & 0.011 \\
\hline Smoking, \% & $14 \%$ & $14 \%$ & 0.926 \\
\hline Systolic blood pressure, mean $( \pm S D)$ & $141.2 \pm 24.4$ & $135.0 \pm 28.7$ & 0.021 \\
\hline Diastolic blood pressure, mean $( \pm S D)$ & $75.6 \pm 13.0$ & $74.5 \pm 17.1$ & 0.402 \\
\hline Diabetes mellitus, $\%$ & $21 \%$ & $23 \%$ & 0.632 \\
\hline Hypercholesterolemia, \% & $27 \%$ & $30 \%$ & 0.926 \\
\hline Prior cardiac event (total) , \% & $32 \%$ & $38 \%$ & 0.246 \\
\hline Prior stroke (CVA) , \% & $39 \%$ & $44 \%$ & 0.315 \\
\hline \multicolumn{4}{|l|}{ Comorbidities } \\
\hline Heart failure, $\%$ & $31 \%$ & $43 \%$ & 0.017 \\
\hline Impaired kidney disease, $\%$ & $10 \%$ & $17 \%$ & 0.066 \\
\hline COPD, $\%$ & $16 \%$ & $20 \%$ & 0.304 \\
\hline Dementia, \% & $57 \%$ & $57 \%$ & 0.956 \\
\hline \multicolumn{4}{|l|}{ (hs-)cTn concentrations } \\
\hline Hs-cTnT concentrations (ng/L), median (IQR) & $19.6(13.9-29.1)$ & $23.2(16.8-41.0)$ & 0.001 \\
\hline Hs-cTnl concentrations (ng/L), median (IQR) & $6.2(3.9-12.1)$ & $8.5(5.6-16.7)$ & 0.001 \\
\hline cTnl concentrations (ng/L), median (IQR) & $4.0(2.0-8.0)$ & $6.0(3.0-13.0)$ & 0.001 \\
\hline
\end{tabular}


SUPPLEMENTAL TABLE 4. Cox-regression analysis to examine the prognostic value of hs-cTnT (Roche), hscTnl (Abbott) and cTnl (Beckman) for all-cause mortality when adjusted for significant risk factors.

$$
\text { HR }(95 \% \mathrm{Cl})
$$

\begin{tabular}{rrl}
\hline Ln(hs-cTnT) (Roche) & & \\
\hline Model 1 & $1.58(1.17-2.15)$ & 0.003 \\
Model 2 & $1.50(1.06-2.14)$ & 0.024 \\
\hline Ln(hs-cTnl) (Abbott) & & 0.139 \\
\hline Model 1 & $1.10(0.97-1.24)$ & 0.467 \\
Model 2 & $1.06(0.91-1.22)$ & \\
\hline Ln(cTnl) (Beckman) & & 0.018 \\
\hline Model 1 & $1.15(1.02-1.29)$ & 0.103 \\
\hline
\end{tabular}

Model 1, adjusted for age, gender, BMI, systolic BP; Model 2, adjusted for age, gender, BMI, systolic BP, presence of heart failure, presence of impaired kidney disease (medical history), eGFR. 



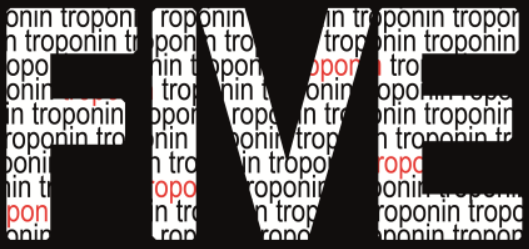

\section{ACUTE EFFECTS OF CONVENTIONAL AND EXTENDED HEMODIALYSIS AND HEMODIAFILTRATION ON HIGH-SENSITIVITY CARDIAC TROPONINS}

Cardinaels $E^{*}$, Cornelis $T^{*}$, van der Sande F, Leunissen K, van Dieijen-Visser M, Mingels A, Kooman J and Bekers $\mathrm{O}$.

*, both authors contributed equally

Clin Chem Lab Med, 2015 [in press] 



\section{Introduction}

Elevated concentrations of cardiac troponin $\mathrm{T}$ (cTnT; 37kDa) and I (cTnl; $24 \mathrm{kDa}$ ), and the $\mathrm{N}$-terminal fragment of the prohormone brain-type natriuretic peptide (NTproBNP; 8.5kDa) have been reported in end-stage renal disease patients and are associated with cardiovascular morbidity and mortality [1, 2]. Also, cTnT, cTnl and NTproBNP correlate with glomerular filtration rate, suggesting a significant role of renal removal and consequences for the clinical interpretation of these cardiac markers [3]. The influence of hemodialysis (HD) on cardiac biomarkers itself remains contradictory. Previous studies have demonstrated intradialytic decreases [4] and increases in NTproBNP [5]. Also, significant reductions as well as increases in cTnT and cTnl concentrations have been reported following dialysis [5-8]. These differences can be attributed to the use of different dialysis modalities and membrane filters $[5,8]$.

Currently, most patients are treated with 4-hour high-flux HD. However, it has been shown that uremic toxins were better removed when patients were treated with hemodiafiltration (HDF) and extended 8-hour dialysis treatments resulted in more hemodynamic stability [9]. The aim of the present study was to assess the acute effects of conventional and extended high-flux HD and HDF on cardiac biomarkers in the same population as previously described [9], especially focusing on high-sensitivity (hs-) cTnT and hs-cTnl results.

\section{Materials and methods}

\section{Study population}

Prevalent conventional HD patients were recruited receiving chronic conventional HD treatments, without residual urine production and acute illness such as infection or cardiovascular events. All patients underwent one mid-week session in random order: 4-hour HD (HD4), 8-hour HD (HD8), 4-hour online HDF (HDF4) and 8-hour online HDF (HDF8) sessions; with a 2-week interval between the study sessions. Between the study sessions, these patients received conventional HD (HD4) treatments. All treatments were performed with the Fresenius 5008 Therapy System (Fresenius Medical Care). High-flux FX80 and FX800 dialyzers (both Fresenius) were used for HD and HDF, respectively. 
Blood flow and dialysate flow were 300 and $600 \mathrm{~mL} / \mathrm{min}$, respectively. Substitution flow was $83.3 \mathrm{~mL} / \mathrm{min}$ to achieve a total substitution volume of $15 \mathrm{~L}$ for HDF4 and 30L for HDF8. Dialysate composition was $1.5 \mathrm{mmol} / \mathrm{L}$ calcium, 2 $\mathrm{mmol} / \mathrm{L}$ potassium, $136-138 \mathrm{mmol} / \mathrm{L}$ sodium and $35-38 \mathrm{mmol} / \mathrm{L}$ bicarbonate and remain unchanged during the study period. This study (NL34908.068.10/MEC10-2-098) was approved by the local ethics committee, and written informed consent was obtained from each patient.

\section{Biochemical measurements}

Blood was drawn from the inlet bloodlines in serum tubes, immediately before and after dialysis. Serum samples were aliquoted, stored at $-80^{\circ} \mathrm{C}$ and thawed prior to analysis. For HDF8, hs-cTnl and NTproBNP could not be measured in 3 patients due to a lack of sufficient serum. cTnT was measured using the hscTnT assay (Roche Diagnostics), with a limit of detection at $5 \mathrm{ng} / \mathrm{L}$ and $10 \%$ coefficient of variation (CV) cutoff at $13 \mathrm{ng} / \mathrm{L}$ and $\mathrm{CTnl}$ was measured with the ARCHITECT hs-cTnl assay (Abbott Diagnostics) with a limit of detection of $2.3 \mathrm{ng} / \mathrm{L}$ and $10 \% \mathrm{CV}$ cutoff at $4 \mathrm{ng} / \mathrm{L}$. NTproBNP concentrations were assessed using the ProBNP-II assay (Roche) with a limit of detection of $0.6 \mathrm{pmol} / \mathrm{L}$ and $\mathrm{CV}$ of $6.8 \%$ at $8.78 \mathrm{pmol} / \mathrm{L}$. Pre- and post-dialysis measurements were performed using the same assay on the same day. Post-dialysis concentrations were corrected for hemoconcentration by ultrafiltration using serum total protein and albumin measurements (Cobas 8000 analyzer, Roche Diagnostics).

\section{Statistical analysis}

Reduction ratios were calculated as follows: $R R=[1$-(pre-dialysis concentration/post-dialysis concentration)]. Differences in pre- versus postdialysis concentrations and reduction ratios $(R R)$ were analyzed using the Wilcoxon signed rank test and pair-wise ANOVA, respectively. Two sided $\mathrm{p}$ values of $<0.05$ were considered statistically significant. Statistical analysis was performed with IMB SPSS 20.0 statistics program. 


\section{Results}

\section{Baseline characteristics}

Thirteen patients completed the study, who were on dialysis for a mean period of $49( \pm 29)$ months. Mean age of the patients was $53.6( \pm 20.4)$ years and 10 patients were males. During the study period, these patients were clinically stable. Additional specifications regarding this study are summarized in SUPPLEMENTAL TABLE 1 and have been previously published [9].

SUPPLEMENTAL TABLE 1. Baseline characteristics as previously published by Cornelis et al [9]

\begin{tabular}{l|cccc} 
Characteristic & HD4 & HD8 & HDF4 & HDF8 \\
\hline Effective dialysis time $(\mathrm{min})$ & $246 \pm 4$ & $486 \pm 2^{\star \S}$ & $245 \pm 3$ & $487 \pm 6^{\star} \S$ \\
Blood volume (L) & $67.9 \pm 1.6$ & $134.9 \pm 2.0^{\star \S}$ & $68.5 \pm 1.1$ & $135.3 \pm 1.4^{\star \S}$ \\
Blood flow (mL/min) & $283 \pm 7$ & $287 \pm 4$ & $286 \pm 5$ & $288 \pm 3$ \\
Dialysate flow $(\mathrm{mL} / \mathrm{min})$ & $569 \pm 24$ & $576 \pm 8$ & $578 \pm 7$ & $572 \pm 11$ \\
Dialysate temperature $\left({ }^{\circ} \mathrm{C}\right)$ & $35.9 \pm 0.4$ & $35.9 \pm 0.4$ & $35.8 \pm 0.4$ & $36.0 \pm 0.4$ \\
Total ultrafilatration $(\mathrm{L})$ & $1.98 \pm 0.76$ & $2.31 \pm 0.82$ & $1.77 \pm 0.63$ & $2.21 \pm 0.81$ \\
Ultrafiltration rate $(\mathrm{mL} / \mathrm{h})$ & $500 \pm 193$ & $295 \pm 111^{\star \S}$ & $449 \pm 160$ & $265 \pm 83^{\star \S}$ \\
Substitution volume $(\mathrm{L})$ & - & - & $14.6 \pm 1.1$ & $29.7 \pm 0.4 \S$ \\
Single pool Kt/ $\mathbf{V}$ & $1.49 \pm 0.21$ & $3.30 \pm 0.54^{\star \S}$ & $1.60 \pm 0.30$ & $3.34 \pm 1.05^{\star \S}$ \\
Equilibrated Kt/V & $1.36 \pm 0.26$ & $3.09 \pm 0.51^{\star \S}$ & $1.41 \pm 0.26$ & $3.14 \pm 0.99^{\star} \S$ \\
\hline
\end{tabular}

$* \mathrm{P}<0.05$ versus HD4; $\S \mathrm{p}<0.05$ versus HDF4

\section{Influence of hemodialysis and hemodiafiltration on hs-cTnT, hs-cTnl and NTproBNP}

Median hs-cTnT concentrations were considerably higher than hs-cTnl concentrations before and after hemodialysis (TABLE 1). Moreover, all predialysis hs-cTnT concentrations were above the diagnostic cut-off of $14 \mathrm{ng} / \mathrm{L}$. In contrast, only three patients had elevated hs-cTnl concentrations (>26.2ng/L). Also, elevated NTproBNP concentrations (>35pmol/L) were measured in all patients. 
No significant differences were found between pre-dialysis hs-cTnT, hs-cTnl and NTproBNP concentrations for different dialysis-modalities $(p>0.05)$.

TABLE 1. hs-cTnT, hs-cTnl and NTproBNP concentrations pre and post dialysis, corrected for hemoconcentration, per modality.

\begin{tabular}{|c|c|c|c|c|}
\hline Hs-cTnT & modality & $\begin{array}{l}\text { hs-cTnT } \text { pre }_{\text {(ng/L); }} \\
\text { Median (IQR) }\end{array}$ & $\begin{array}{l}\text { hs-cTnT } \text { post }_{\text {(ng/L); }} \\
\text { Median (IQR) }\end{array}$ & $p$-value \\
\hline & HD4 & $43.1(36.1-62.8)$ & $48.0(31.4-77.1)$ & 0.158 \\
\hline & HD8 & $41.6(26.8-57.8)$ & $35.9(19.5-61.4)$ & 0.534 \\
\hline & HDF4 & $46.0(37.2-65.0)$ & $41.8(24.2-60.3)$ & 0.013 \\
\hline & HDF8 & $44.1(33.0-57.3)$ & $33.9(20.7-61.4)$ & 0.004 \\
\hline \multirow[t]{5}{*}{ Hs-cTnl } & modality & $\begin{array}{l}\text { hs-cTnl }{ }_{\text {pre }}(\mathrm{ng} / \mathrm{L}) \\
\text { Median (IQR) }\end{array}$ & $\begin{array}{l}\text { hs-cTnl }\left.\right|_{\text {post }}(\mathrm{ng} / \mathrm{L}) \\
\text { Median (IQR) }\end{array}$ & $\mathrm{p}$-value \\
\hline & HD4 & $11.4(8.2-17.7)$ & $12.6(8.6-22.2)$ & 0.347 \\
\hline & HD8 & $17.2(7.4-26.5)$ & $13.7(6.7-24.0)$ & 0.203 \\
\hline & HDF4 & $18.6(7.0-24.6)$ & $19.0(6.1-29.1)$ & 0.917 \\
\hline & HDF8* & $22.4(6.7-34.0)$ & $12.8(5.7-29.1)$ & 0.028 \\
\hline \multirow[t]{5}{*}{ NTproBNP } & modality & $\begin{array}{c}\text { NTproBNP pre (pmol/L); } \\
\text { Median (IQR) }\end{array}$ & $\begin{array}{c}\text { NTproBNP } \\
\text { Mestian (IQR) }\end{array}$ & $p$-value \\
\hline & HD4 & $427.3(283.6-754.6)$ & $407.2(220.8-1112)$ & 0.034 \\
\hline & HD8 & $393.0(234.8-574.8)$ & $218.2(125.4-349.4)$ & 0.008 \\
\hline & HDF4 & $458.9(256.1-1170)$ & $249.3(117.3-591.4)$ & 0.002 \\
\hline & HDF8* & $308.6(245.2-512.9)$ & $102.2(59.8-179.6)$ & 0.008 \\
\hline
\end{tabular}

*based on serum samples of 10/13 patients 
Generally, decreases in hs-cTnT, hs-cTnl as well as NTproBNP were found immediately after dialysis (TABLE 1). However, no significant differences were observed between pre- and postdialysis hs-cTnT and hs-cTnl concentrations after HD4 and HD8 (TABLE 1; $\mathrm{p}>0.05)$. In contrast, HDF had a highly significant effect on hs-cTnT concentrations $(p<0.01)$, especially following HDF8. Also hs-cTnl demonstrated a significant decrease after HDF8 but not HDF4 (TABLE 1). For NTproBNP, significant intradialytic decreases following all modalities were observed, with the highest reduction after HDF8 (TABLE 1 and FIGURE 1).

When comparing HD4 to HDF8,
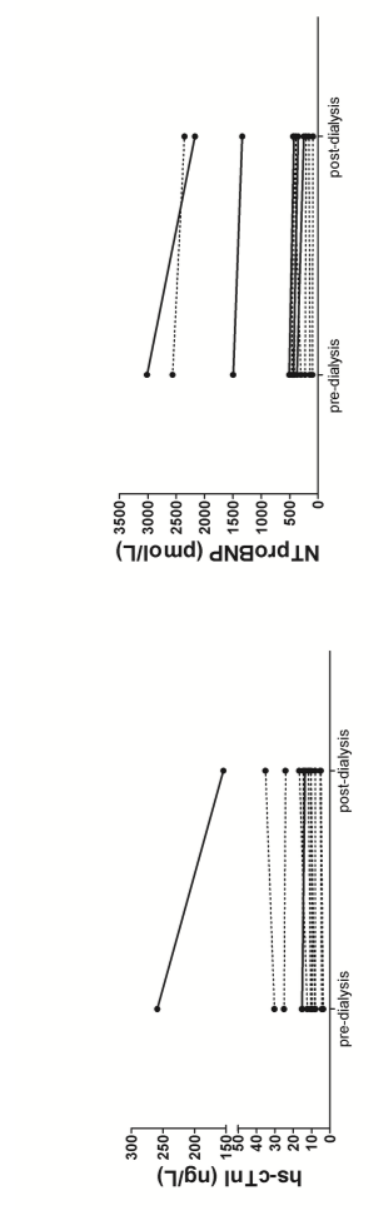

more patients demonstrated a significant $(>10 \%)$ decrease in hscTnT, hs-cTnl and NTproBNP concentrations following HDF8 (SUPPLEMENTAL FIGURE 1).

SUPPLEMENTAL FIGURE 1 . Individual changes in hs-cTnT, hs-cTnl and NTproBNP concentrations following either HD4 and HDF8-treatments. Full lines indicate a $>10 \%$ decrease pre versus post-dialysis, while dotted lines indicate less than $10 \%$ reduction.
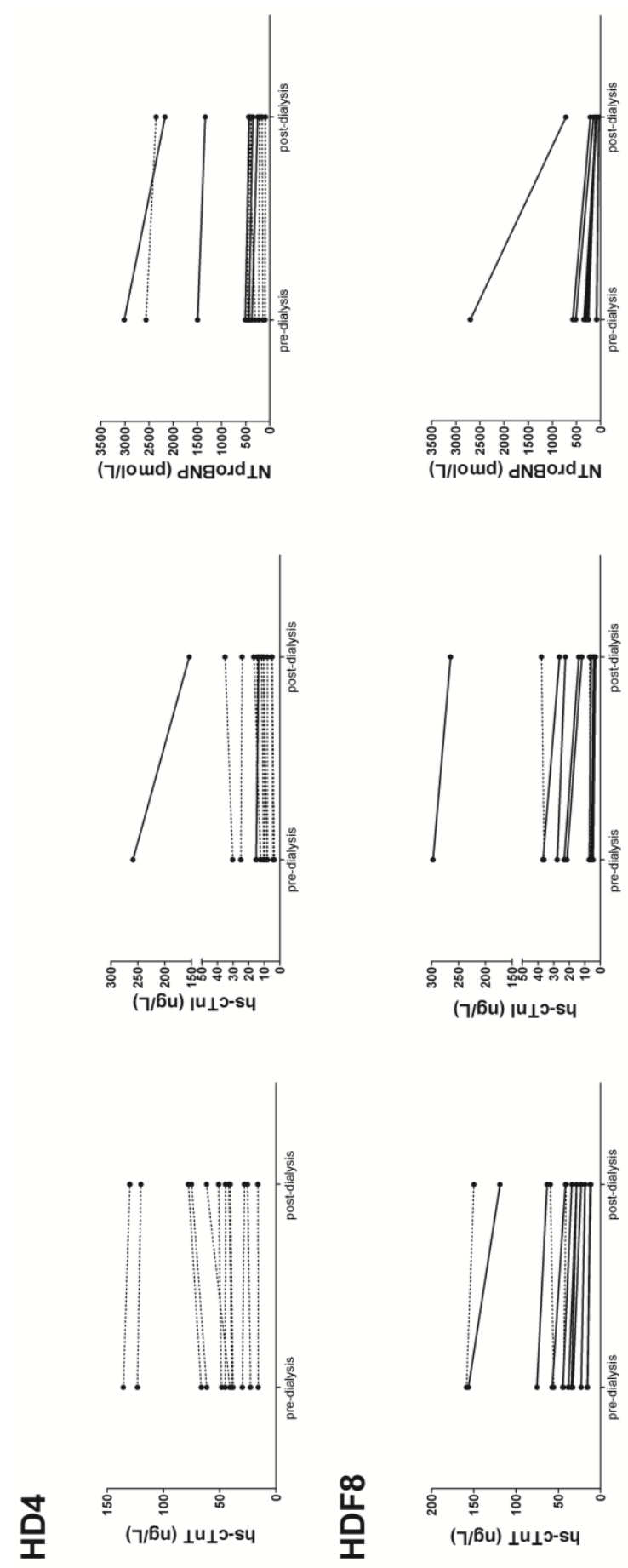
For hs-cTnT, significantly higher RRs were established following HDF compared to HD (FIGURE 1). Interestingly, RR were significantly higher for hs-cTnl after HD8 and HDF8 in comparison to HD4 and HDF4. Overall the highest RR were obtained following HDF8, although mean RR were still lower for hs-cTnT $(20.4 \pm 8.8 \%)$ and hs-cTnl $(23.0 \pm 16.2 \%)$ in comparison to NTproBNP $(64.2 \pm 9.5 \%)$ and other uremic toxins $(50-90 \%$; [9]).

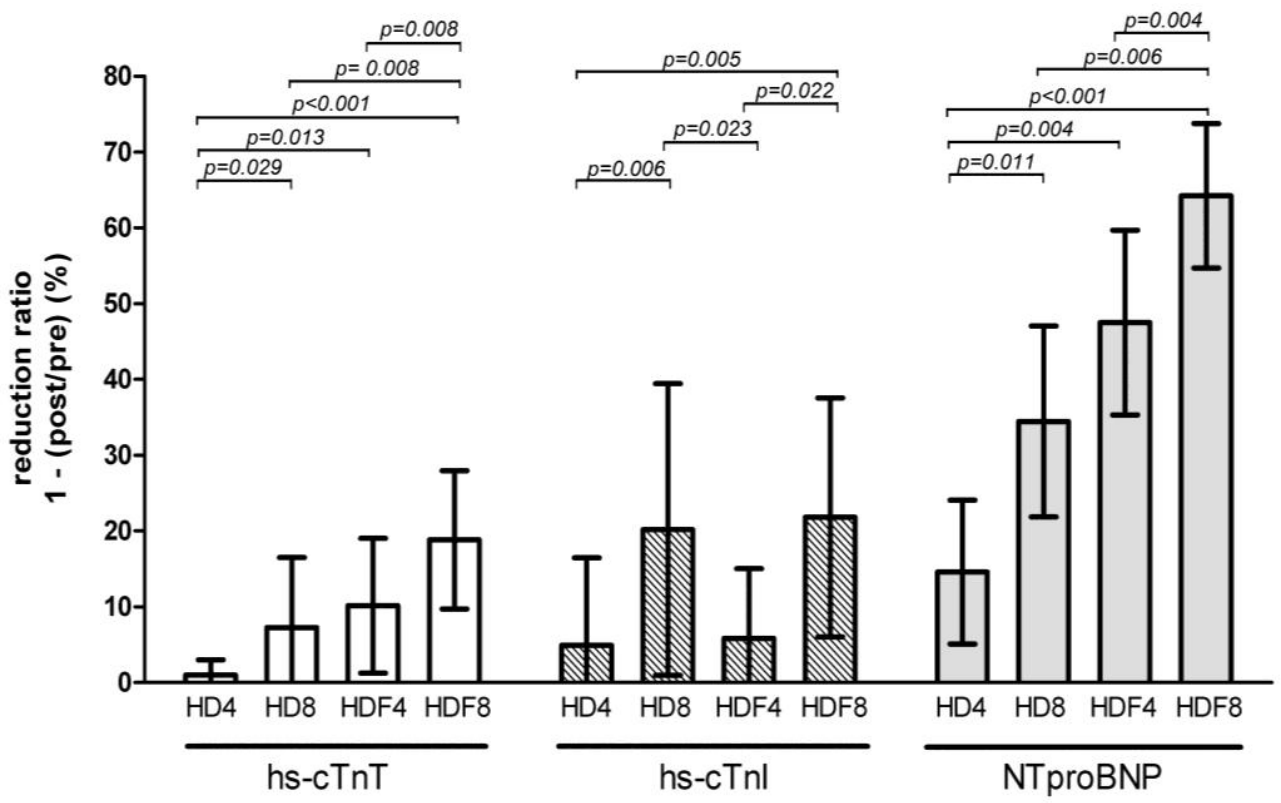

FIGURE 1. Mean $( \pm S D)$ reduction ratios of hs-cTnT, hs-cTnl and NTproBNP, corrected for hemoconcentration, following 4 and 8 hour hemodialysis and hemodiafiltration.

Identical results were found when hs-cTnT, hs-cTnl and NTproBNP concentrations were corrected for hemoconcentration by ultrafiltration using albumin concentrations (data not shown). 


\section{Discussion}

This is the first study to reveal the effects of extended hemodialysis and hemodiafiltration on cardiac markers. Here, we clearly demonstrate a significant reduction in hs-cTnT, hs-cTnl and NTproBNP concentrations following HDF, although RR remained relatively low for both hs-cTn measurements (<25\%).

Recently it was shown that hs-cTnT concentrations display a diurnal variation, with peak hs-cTnT concentrations in the morning that gradually decrease during the day [10]. Herein, we found higher RR for hs-cTnT following 4 hour HDF in comparison to 8 hour HD. Consequently, we can conclude that dialysis treatments have an additional effect on hs-cTnT concentrations. However, whether these cTn reductions are due to clearance, still has to be elucidated by measuring in dialysate samples.

Interestingly, the reduction of cTnT was more influenced by the use of convection in comparison to cTnl [9]. In addition, 8-hour dialysis treatments had the largest effects on the RR of cTnl. Whether this is the consequence of differences in cTn release or in clearance from the blood stream merits further research.

In conclusion, this study demonstrated a clear effect of different dialysis modalities on cardiac biomarkers with highest RR's during HDF8. Clinicians should be aware of this intradialytic reduction in cardiac biomarkers when interpreting serial results, especially in the diagnostic context of an acute myocardial infarction. The long-term effects of these dialysis modalities on the levels of cardiac biomarkers should be addressed in future studies.

\section{Acknowledgments}

We sincerely thank Abbott Diagnostics for providing the hs-cTnl kits. The authors also acknowledge the laboratory assistance of V.W.V.C. Kleijnen. This work was supported by a research grant from Stichting De Weijerhorst to M.P v.D-V. 


\section{References}

1. Apple, F.S., M.M. Murakami, L.A. Pearce, et al., Multi-biomarker risk stratification of Nterminal pro-B-type natriuretic peptide, high-sensitivity C-reactive protein, and cardiac troponin T and I in end-stage renal disease for all-cause death. Clin Chem, 2004. 50(12): p. 2279-85.

2. Mishra, R.K., Y. Li, C. DeFilippi, et al., Association of cardiac troponin T with left ventricular structure and function in CKD. Am J Kidney Dis, 2013. 61(5): p. 701-9.

3. deFilippi, C., S.L. Seliger, W. Kelley, et al., Interpreting cardiac troponin results from highsensitivity assays in chronic kidney disease without acute coronary syndrome. Clin Chem, 2012. 58(9): p. 1342-51.

4. Wahl, H.G., S. Graf, H. Renz, et al., Elimination of the cardiac natriuretic peptides B-type natriuretic peptide (BNP) and N-terminal proBNP by hemodialysis. Clin Chem, 2004. 50(6): p. 1071-4.

5. Sommerer, C., S. Heckele, V. Schwenger, et al., Cardiac biomarkers are influenced by dialysis characteristics. Clin Nephrol, 2007. 68(6): p. 392-400.

6. Hassan, H.C., K. Howlin, A. Jefferys, et al., High-sensitivity troponin as a predictor of cardiac events and mortality in the stable dialysis population. Clin Chem, 2014. 60(2): p. 389-98.

7. Assa, S., R.T. Gansevoort, R. Westerhuis, et al., Determinants and prognostic significance of an intra-dialysis rise of cardiac troponin I measured by sensitive assay in hemodialysis patients. Clin Res Cardiol, 2013. 102(6): p. 439-45.

8. Lippi, G., N. Tessitore, M. Montagnana, et al., Influence of sampling time and ultrafiltration coefficient of the dialysis membrane on cardiac troponin I and T. Arch Pathol Lab Med, 2008. 132(1): p. 72-6.

9. Cornelis, T., F.M. van der Sande, S. Eloot, et al., Acute hemodynamic response and uremic toxin removal in conventional and extended hemodialysis and hemodiafiltration: a randomized crossover study. Am J Kidney Dis, 2014. 64(2): p. 247-56.

10. Klinkenberg, L.J., J.W. van Dijk, F.E. Tan, et al., Circulating cardiac troponin T exhibits a diurnal rhythm. J Am Coll Cardiol, 2014. 63(17): p. 1788-95. 




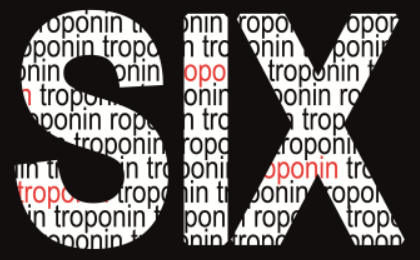

\section{TIME-DEPENDENT DEGRADATION PATTERN OF CARDIAC TROPONIN T FOLLOWING ACUTE MYOCARDIAL INFARCTION}

Cardinaels E*, Mingels A*, van Rooij T, Collinson P, Prinzen F and van DieijenVisser M.

*both authors contributed equally

Clin Chem 2012;60:1098-1104 


\section{Abstract}

\section{Background}

Cardiac troponin $\mathrm{T}$ (cTnT) is widely used for the diagnosis of acute myocardial infarction (AMI). However, it is still unclear whether degraded cTnT forms circulate in the patient's blood. We therefore aimed to elucidate which cTnT forms are detected by the clinical assay.

\section{Methods}

Separation of cTnT forms by gel filtration chromatography (GFC) was performed in sera from 13 AMI patients to examine cTnT degradation. The GFC eluates were subjected to Western blot analysis, using the original antibodies from the Roche immunoassay to mimic the clinical cTnT assay. To investigate the degradation pattern with time, standardized serum samples of $18 \mathrm{AMI}$ patients collected 0-72 hours post-admission were analyzed by Western blot analysis.

\section{Results}

GFC analysis of AMI patients' sera revealed two cTnT peaks with retention volumes of 5 and $11 \mathrm{~mL}$. Western blot analysis identified these peaks as cTnT fragments of 29 and 14-18 kDa, respectively. Furthermore, the performance of direct Western blots on standardized serum samples, demonstrated a timedependent degradation pattern of cTnT, with fragments ranging between 14 and $40 \mathrm{kDa}$. Intact cTnT (40 kDa) was only present in 3 patients within the first 8 hours after hospital admission.

\section{Conclusion}

These results demonstrate that the Roche cTnT immunoassay detects intact as well as degraded cTnT forms in AMI patients' sera during the period of diagnostic testing. Moreover, following AMI, cTnT is degraded in a timedependent pattern. 


\section{Introduction}

Cardiac troponin $\mathrm{T}$ (cTnT) and cardiac troponin I (cTnl) are superior to other cardiac markers because of their unique cardiospecificity. Consequently, they are considered to be the biomarkers of first choice to diagnose and monitor an acute myocardial infarction (AMI) [1]. Owing to patent restrictions only one cTnT assay (Roche Diagnostics) is currently commercially available, as compared with over twenty different assays for cTnl [2]. In addition, according to a recent survey, the Roche cTnT assay is used in half of European laboratories [3]. Therefore, characterization of cTnT, as detected by this assay, is important to better define its role in clinical decision making.

The cTn T-I-C complex is structurally bound predominantly to the myofibrils with only a minor fraction of cTnT and cTnl (6-8\%) present in the cytoplasm as soluble intact protein [4, 5], although this finding has been disputed [6]. Typically, AMI patients undergoing rapid reperfusion demonstrate a first CTnT peak concentration within 24 hours after onset of symptoms, which has been attributed to the fast release of cytosolic cTnT [4, 7]. This peak is followed by a second and persistent cTnT elevation which remains present for 7-14 days and is generally thought to represent the relatively slow dissociation of cTnT from the sarcomeres and release from the cells during necrosis. There is limited knowledge on the posttranslational modifications of cTnT after myocardial damage, in particular degradation of cTnT. On the other hand, degradation products of cTnl have been extensively reported [8-11] and even associated with post-ischemic contractile dysfunction $[12,13]$.

Previously, we have reported the presence of cTnT fragments on Western blots of human serum from renal failure and post AMI patients using different antibodies as the clinical assay $[14,15]$. Other investigations have not confirmed these findings and found only intact cTnT on gel filtration chromatography (GFC) of serum from such patients [16, 17]. To date it is still not clear which cTnT forms circulate in the serum of AMI patients and if they are detected by the Roche cTnT assay.

In this study, we aimed to elucidate whether degraded cTnT forms are present in the circulation following ischemic myocardial damage. We examined AMI patients' sera using a novel approach. Firstly, the patients' sera were 
fractionated by GFC, as in previous studies [16, 17]. Secondly, these GFC fractions were subjected to a unique Western blotting technique, in which the capture and detector antibodies of the commercial cTnT assay were incorporated to mimic the working principle of this assay. In a second AMI population, we aimed to characterize the changes of the molecular forms of cTnT with time. Serum samples collected for each patient at standardized time points after symptom onset were subjected directly to this Western blotting technique.

\section{Materials and Methods}

\section{Study populations}

GFC followed by Western blotting was performed on residual serum samples from 13 patients with acute myocardial infarction followed by rapid revascularization. Blood samples were obtained from the routine laboratory at two time points $3( \pm 1)$ as well as $15( \pm 2)$ hours after admission to the emergency department of Maastricht University Medical Center) and frozen at $-80^{\circ} \mathrm{C}$. One serum sample was missing from each of two AMI patients, one at 3 hours and one at 15 hours post-admission. As a result, $12 \mathrm{AMI}$ serum samples were analyzed for both time points.

The molecular changes of cTnT with time and their subsequent effect on infarct size estimation were examined in a second well-characterized AMI population. Serum samples of 18 ST-segment elevation myocardial infarction (STEMI) patients were collected at hospital admission and at standardized time points: 4 , $8,12,24,36,48,60$, and 72 hours after hospital admission and immediately stored at $-80^{\circ} \mathrm{C}$. These patients were enrolled in the control group of a clinical trial investigating the effects of ventricular pacing during revascularization, and thus receiving standard treatment (http://clinicaltrials.gov/ ct2/show/NCT00409604). Inclusion criteria for the study were as follows: age $>18$ years, presentation with their first myocardial infarction, admitted to hospital $3( \pm 1)$ hours from symptom onset, treated with percutaneous coronary intervention $(\mathrm{PCl})$ and exhibiting a typical release pattern of cTnT, creatine kinase (CK) and lactate dehydrogenase (LD). 
The study was conducted according to the principles of the Declaration of Helsinki and approved by the local ethics committees. Informed consent was obtained from the patients.

\section{Biochemical testing}

cTnT was measured on the Elecsys 2010 (Roche) using the 4th generation cTnT assay with a limit of detection $<0.01 \mu \mathrm{g} / \mathrm{L}$ and $10 \%$ CV cutoff at $0.03 \mu \mathrm{g} / \mathrm{L}$. NT-proBNP was also measured on the Elecsys 2010 (Roche) with limit of detection $5.08 \mathrm{ng} / \mathrm{L}(0.6 \mathrm{pmol} / \mathrm{L})$ and inter-assay CV $6.8 \%$ at $74.4 \mathrm{ng} / \mathrm{L}$ (8.78 $\mathrm{pmol} / \mathrm{L}$ ). Albumin was measured on the Synchron LX20 (Beckman Coulter) using the microalbumin assay with measuring range of $2.0-970 \mathrm{mg} / \mathrm{L}$. CK and LD were measured on the Synchron LX20 (Beckman) as well. cTnl was measured using the Axsym troponin I-Adv assay (Abbott Laboratories) with the $10 \% \mathrm{CV}$ cutoff at $0.16 \mu \mathrm{g} / \mathrm{L}$. All assay characteristics were given by the manufacturer.

\section{Fractionation by GFC}

GFC was performed on a HP 1100 system (Agilent Technologies) equipped with a $1.6 \times 60 \mathrm{~cm}$ Sephacryl-S100 column (GE Healthcare) and a diode array detector. The column was equilibrated with $0.26 \mathrm{~mol} / \mathrm{L} \mathrm{NaCl}, 2.5 \mathrm{mmol} / \mathrm{L} \mathrm{CaCl} 2$. $2 \mathrm{H} 2 \mathrm{O}, 0.02 \mathrm{~mol} / \mathrm{L}$ Tris, $6 \mathrm{mmol} / \mathrm{L} \mathrm{NaN3}$ and $1 \mathrm{~g} / \mathrm{L}$ bovine serum albumin buffer, $\mathrm{pH} 7.4$ [16] and operated at $0.5 \mathrm{~mL} / \mathrm{min}$. The void volume (V0) determination and calibration was conducted using the Gel Filtration Calibration kit (GE Healthcare) containing Dextran Blue (molecular weight (MW), 2000 kDa), conalbumin (MW, $75.0 \mathrm{kDa}$ ), ovalbumin (MW, $43.0 \mathrm{kDa}$ ), carbonic anhydrase (MW, $29.0 \mathrm{kDa}$ ) and ribonuclease (MW, $13.7 \mathrm{kDa})$, supplemented with myoglobin (MW, $16.9 \mathrm{kDa}$, Sigma). Dextran Blue $(1 \mathrm{mg} / \mathrm{ml})$ and globular protein standards (3-4 mg/ml) were dissolved in running buffer, $0.5 \mathrm{~mL}$ was loaded on the column, and absorbance was studied at $280 \mathrm{~nm}$, all in duplo. V0 was determined to be $37 \mathrm{~mL}$.

In addition, purified human cTn T-I-C complex (MW, $77 \mathrm{kDa}$; Hytest) and purified free cTnT, cTnl, and troponin C (TnC) (MW, 37, 24 and $18 \mathrm{kDa}$, respectively; all Advanced ImmunoChemical) were dissolved as prescribed by 
the manufacturer and were further diluted in running buffer or spiked in serum pool of healthy volunteers (cTnT $<0.01 \mu \mathrm{g} / \mathrm{L}$ and cTnl $<0.03 \mu \mathrm{g} / \mathrm{L}$ ).

To obtain the standard calibration curve, the gel-phase distribution coefficient $\left(\mathrm{K}_{\mathrm{av}}=\mathrm{V}_{\mathrm{R}} /\left(\mathrm{V}_{\mathrm{c}}-\mathrm{V}_{0}\right)\right)$ was plotted against the logarithm of MW of globular proteins and purified $\mathrm{cTn}$ standards. $\mathrm{V}_{\mathrm{R}}$ signifies the retention volume adjusted to void volume, while $\mathrm{V}_{\mathrm{c}}$ indicated column volume and corresponded to $120 \mathrm{~mL}$.

For serum samples, $1.0 \mathrm{~mL}$ was loaded on the column. Serum albumin (67 kDa) and serum NT-proBNP were used as internal standards in the patients' sera.

For each sample loaded, fractions of $1.25 \mathrm{~mL}$ were collected, kept on ice until immunoassay measurements ( $\leq 1$ hour) and subsequently stored at $-80^{\circ} \mathrm{C}$.

\section{Purification and Western blot detection of cTnT}

Purification and characterization of cTnT was performed using the antibodies from the Roche cTnT-assay (as kindly provided by Roche Diagnostics, www.roche.com). The capture cTnT antibody (M11.7, epitope a.a.r. 136-147) was used for immunoprecipitation of cTnT. To each $\mathrm{mL}$ of magnetic streptavidin-coated dynabeads (Invitrogen) $10 \mu \mathrm{g}$ biotinylated M11.7 was added. Additionally, beads were crosslinked three times with dimethyl pimelimidate. Subsequently, $200 \mu \mathrm{L}$ of serum was precipitated with $50 \mu \mathrm{L}$ of M11.7-coated beads for 1 hour at $4^{\circ} \mathrm{C}$. Due to low cTnT concentrations in the GFC fractions, more sample was added to the beads, ranging from 200-5000 $\mu \mathrm{L}$. In some cases, up to 4 fractions were merged to generate larger volumes. The average cTnT yield after immunoprecipitation was $>90 \%$ (data not shown). After being washed with PBS containing 0.1\% Tween-20 (PBST) the immunoprecipitates were eluted with $1 \mathrm{M}$ glycine $(18 \mu \mathrm{L}), \mathrm{pH} 3$ for $15 \mathrm{~min}$ at $56^{\circ} \mathrm{C}$. (SUPPLEMENTAL FIGURE 1)

As negative controls for immunoblotting of GFC samples and AMI patients' sera, running buffer or the serum pool of healthy volunteers was used, respectively. As positive controls, cTn T-I-C complex was spiked in running buffer and CTnT negative pooled serum, respectively. Only GFC fractions containing sufficiently high cTnT concentrations could be visualized on the immunoblot, corresponding to 9 out of 12 serum samples collected at \pm 15 hours post admission.

Immunoprecipitates were diluted with XT Sample Buffer $(6 \mu \mathrm{L})$ (Bio-Rad Laboratories), heated for $7 \mathrm{~min}$. at $56^{\circ} \mathrm{C}$, and separated on a $12 \%$ Criterion XT 
SDS-PAGE gel (Bio-Rad) together with the Precision Plus Protein Standard (Bio-Rad). After stacking for $15 \mathrm{~min}$ at $100 \mathrm{~V}$ the proteins were resolved for 45 min at $200 \mathrm{~V}$ in XT MOPS electrophoresis buffer (Bio-Rad) and were transferred to the nitrocellulose membrane (Bio-Rad, $0.45 \mu \mathrm{m}$ ) at $100 \mathrm{~V}$ for $1 \mathrm{~h}$ at $4^{\circ} \mathrm{C}$. The membranes were treated with the vacuum SNAP i.d. protein detection system (Millipore) according to manufacturer instructions. Non-specific binding was blocked with $0.5 \%$ non-fat dry milk Blocking-Grade Blocker (Bio-Rad) in PBST. Primary and secondary antibodies were Roche M7 anti-cTnT antibody (epitope a.a.r. $125-131 ; 10 \mu \mathrm{g} / \mathrm{L})$ and goat anti-mouse peroxidase $(0.4 \mu \mathrm{g} / \mathrm{mL}$; DAKO) in PBST, respectively. Subsequently, membranes were incubated for 5 minutes in Super Signal West Femto Substrate (Thermo Scientific) and bands were detected using the ChemiDoc XRS scanner (Bio-Rad) and Quantity One Software (Bio-Rad, Version 4.6.5.).

\section{Statistical Analysis}

Results are presented as means $( \pm S D$ ) or when not normally distributed as medians (interquartile range (IQR)). The Wilcoxon signed rank test was applied to investigate differences in peak-to-peak ratios of the GFC elution profiles obtained 3 and 15 hours post-admission. All statistical analyses were performed using SPSS (Statistical Package for Social Sciences; version 18). A p-value $<0.05$ was regarded as statistically significant.

\section{Results}

\section{Validation of cTnT degradation by GFC followed by Western blotting}

Elution profiles of globular protein standards (MW varying from 16.9 to 75.0 kDa) and purified cTn standards (cTn T-I-C complex, free cTnT, cTnl and TnC) were determined. A schematic overview of the obtained retention volumes adjusted to $V_{0}\left(V_{R}\right)$ is given in FIGURE $1 A$. 

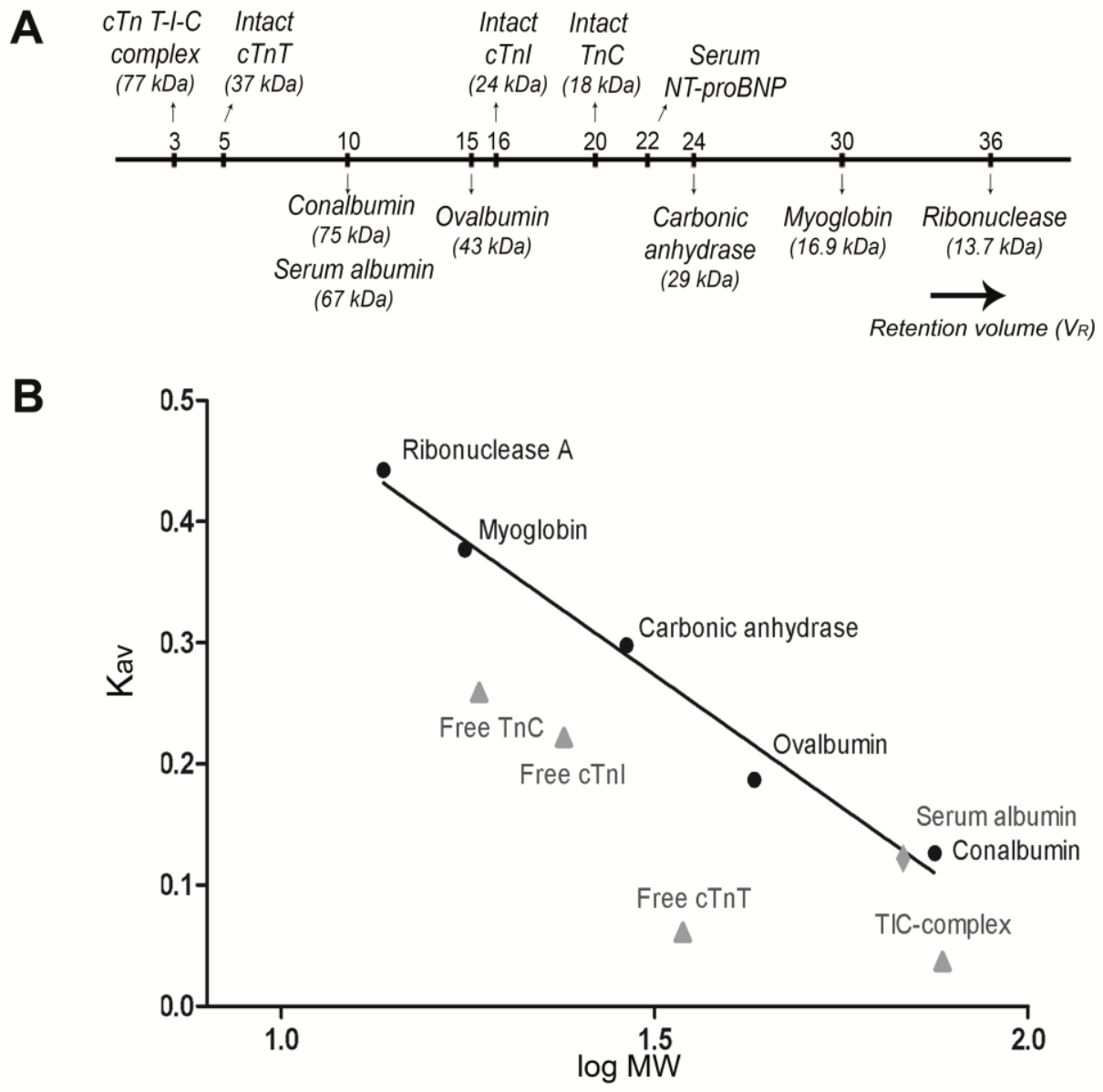

FIGURE 1. Validation of GFC analysis. (A) Schematic overview of the retention volumes obtained for purified cTn standards (cTnT-I-C, cTnT, cTnl, TnC), globular proteins and internal serum standards (serum albumin and NT-proBNP). (B) Standard calibration curve obtained with globular proteins (•) and purified cTn standards ( $\mathbf{\Delta})$. The gel-phase distribution coefficient (Kav=VR/(Vc-VO); Vc=column volume) was plotted against the log molecular weight (MW).

When spiking the intact cTnT standard in cTnT-negative serum (cTnT, 19.9 $\mu \mathrm{g} / \mathrm{L})$, cTnT elutes with $\mathrm{V}_{\mathrm{R}}$ of $5 \mathrm{~mL}$ before the elution of the internal standard serum albumin $\left(\mathrm{V}_{\mathrm{R}}=11 \mathrm{~mL}\right)$ (SUPPLEMENTAL FIGURE 2A). Western blotting of these GFC fractions confirmed that this peak corresponded to intact CTnT (estimated $\mathrm{MW}, \mathrm{MW}_{\text {est }}$ of $40 \mathrm{kDa}$ ) and for a small part to $29 \mathrm{kDa} \mathrm{cTnT}$ fragment, as shown in SUPPLEMENTAL FIGURE 2B. An identical elution profile and immunoblot analysis were obtained when the purified cTnT standard was spiked 
in running buffer (data not shown). For the other purified cTn standards, $V_{R}$ was determined to be $3 \mathrm{~mL}, 16 \mathrm{~mL}$ and $20 \mathrm{~mL}$ for the cTnT-I-C complex, intact cTnl and TnC, respectively. (FIGURE 1A) Moreover, the correlation between $V_{R}$ and $\mathrm{MW}$ of the globular proteins can be represented by a linear calibration curve following the equation $y=-0.44 x+0.93$, as depicted by FIGURE $1 \mathrm{~B}$. In contrast, all cTn proteins (cTnT-I-C complex, free cTnT, cTnl and TnC) deviated clearly from the calibration curve.

Median (IQR) cTnT concentrations measured in serum of AMI patients, subjected to GFC, were 2.17 (5.07) $\mu \mathrm{g} / \mathrm{L}$ and 6.94 (6.46) $\mu \mathrm{g} / \mathrm{L}$ for 3 and 15 hours post-admission, respectively. After GFC separation, overall median (IQR) recovery of loaded cTnT (\%) was 79 (27) \% (SUPPLEMENTAL TABLE 1). The GFC elution profiles of each patient showed two cTnT peaks at an adjusted $V_{R}$ of 5 and $22 \mathrm{~mL}$, eluting before and after the internal standard serum albumin $\left(V_{R}\right.$ $=11 \mathrm{~mL}$ ). As shown in FIGURE 2A and 2B this was observed at 3 as well as 15 hours post-admission, respectively. Western blot analysis using the antibodies of the clinical immunoassay identified the first cTnT peak at $V_{R}$ of $5 \mathrm{~mL}$ as a degradation product of cTnT with a $\mathrm{MW}_{\text {est }}$ of $29 \mathrm{kDa}$ (FIGURE 2C and D). The second peak $\left(\mathrm{V}_{\mathrm{R}}=22 \mathrm{~mL}\right)$ was allocated to smaller cTnT degradation products of $\mathrm{MW}_{\text {est }}$ between 14 and $18 \mathrm{kDa}$. Moreover, there was a significant relative increase in the second peak at 15 hours post admission as compared with 3 hours post-admission (median ratio of cTnT-peak at $22 \mathrm{~mL} / 5 \mathrm{~mL}$ of 0.54 and 1.89 for 3 and 15 hours post-admission respectively; $P=0.001$ ), suggesting a different cTnT degradation pattern that changed with time. 
FIGURE 2. GFC analysis of AMI patients' sera. Elution profiles of AMI patients' sera obtained at (A) 3 and (B) 15 hours post-admission. Median (black) and IQR (grey) CTnT concentrations are shown. Serum albumin and serum NT-proBNP were used as internal standards. (C) CTnT Western blot of the fractions of a representative AMI serum sample obtained 15 hours after hospital admission. $P$, positive control; $\mathrm{M}$, marker; $\mathrm{N}$, negative control. (D) Summary of the molecular forms of CTnT present in AMI serum samples collected 15 hours after hospital admission. Each dot represents a band on the Western blot of a patient.
A 3 hours after admission

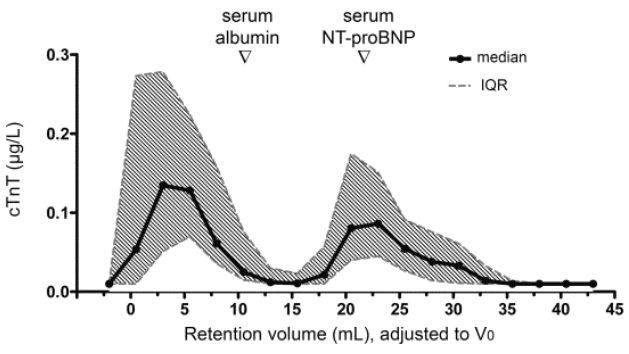

B 15 hours after admission

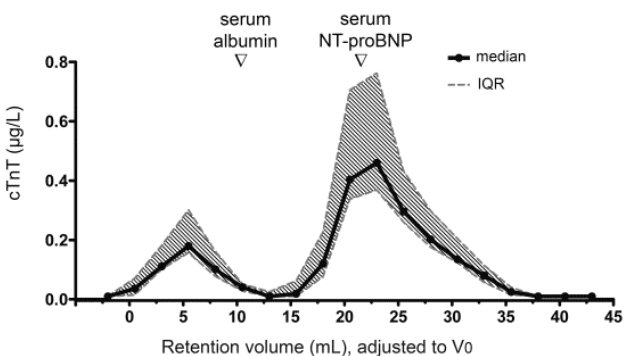

C 15 hours after admission

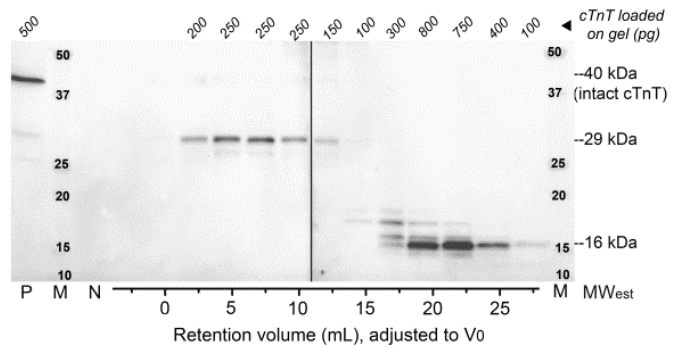

D

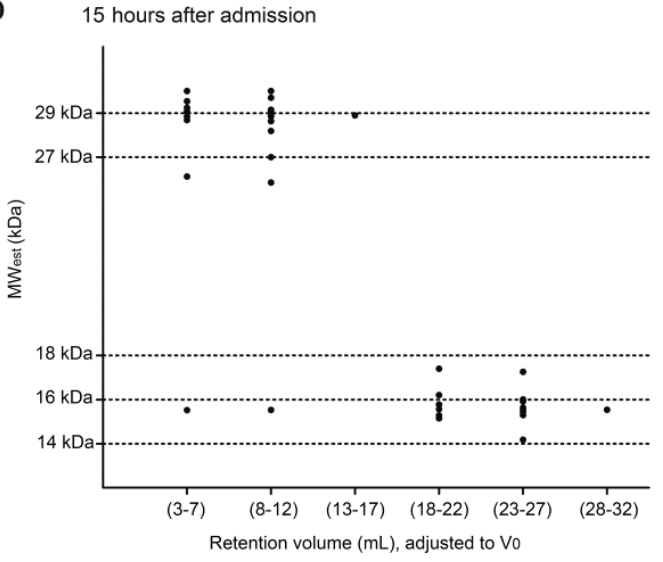




\section{Time course of cTnT degradation at standardized time points}

The release kinetics of CTnT, CK and LD determined in eighteen STEMI patients (66\% males with mean(SD) age 60(9) years (see SUPPLEMENTAL TABLE 2) are shown in FIGURE $3 A$ and SUPPLEMENTAL FIGURES 3 and 4 , respectively. On average, peak cTnT concentrations were reached 8 hours after admission, with a median (IQR) concentration of 6.68 (4.54) $\mu \mathrm{g} / \mathrm{L}$. Furthermore, cTnT levels were still elevated 72 hours post-admission with a median (IQR) concentration of $2.65(1.83) \mu \mathrm{g} / \mathrm{L}$.

FIGURE 3B depicts a representative blot of an AMI patient showing that the antibodies of the clinical assay detected mainly CTnT degradation products in the circulation. As many as five different degradation products were reproducibly identified $\left(\mathrm{MW}_{\text {est }}\right.$ of $14,16,18,27$ and $29 \mathrm{kDa}$ ). Moreover, a breakdown pattern of cTnT was observed over time. Western blot analysis of all patients showed that intact cTnT ( $\mathrm{MW}_{\text {est }}$ of $40 \mathrm{kDa}$ ) was present only in three patients during the first 8 hours after admission (FIGURE 3C). Instead the 29 $\mathrm{kDa}$ fragment of cTnT was detected in highest abundance up to 24 hours postadmission. Afterwards, the larger fragments $\left(\mathrm{MW}_{\text {est }}\right.$ of 27 and $\left.29 \mathrm{kDa}\right)$ disappeared and primarily low-molecular weight fragments between 14 and 18 $\mathrm{kDa}$ were identified. When blotting with an antibody directed against the $\mathrm{C}$ terminus of CTnT, the same degradation pattern for CTnT was visualized. (SUPPLEMENTAL FIGURE 5) On the contrary, when applying an antibody directed against the $\mathrm{N}$ terminus of $\mathrm{cTnT}$, none of the cTnT fragments were detected. 
A

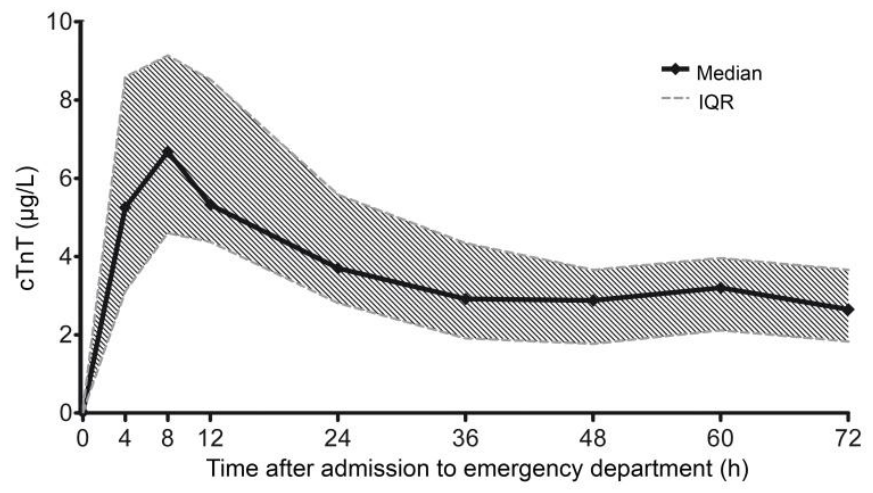

B
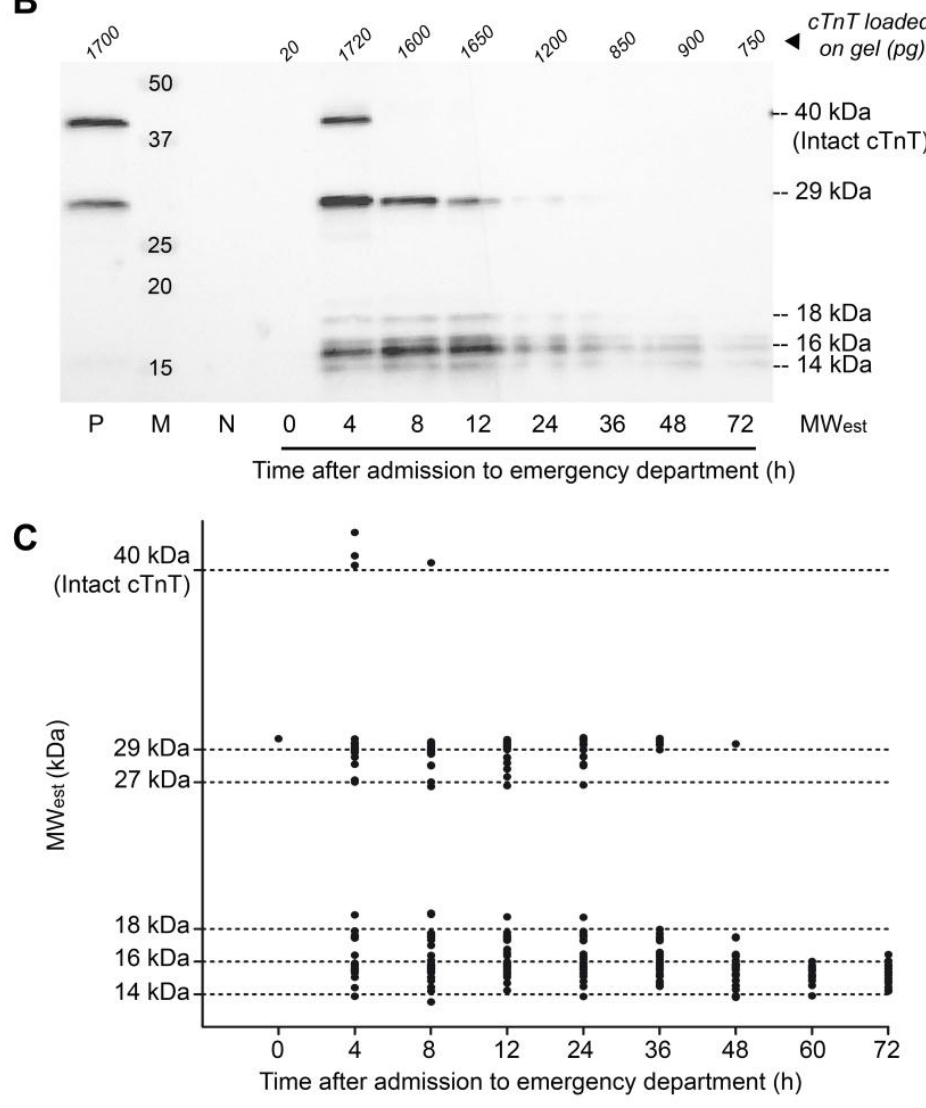

FIGURE 3. Time course of cTnT degradation at standardized time points. (A) Release kinetics of cTnT in AMI patients represented by the median (black) and IQR (grey) cTnT-concentrations. (B) cTnT Western blot of serum samples obtained at standardized time points of a representative $A M I$ patient $P$, positive control; $M$, marker; $\mathrm{N}$, negative control. (C) Summary of the molecular forms of cTnT present after AMI at the standardized time points. Each dot represents a band on the Western blot of a patient. 


\section{Discussion}

The present study shows for the first time that circulating cTnT is highly degraded in cases of AMI followed by rapid revascularization, as demonstrated by fractionation of serum from AMI patients with GFC and Western blotting, using the antibodies of the clinical cTnT assay.

GFC enables detailed characterization of non-covalent complexes present in the patient's serum, but also requires extensive and careful validation. Herein, we report similar elution profiles as previously found by Bates and colleagues [16], although they assigned their main peak to intact cTnT. As determined from the calibration curve, obtained with globular protein standards, the expected adjusted $V_{R}$ for intact cTnT would be $20 \mathrm{~mL}$. However, in this study we clearly demonstrate that purified cTnT, and also other cTn molecules, eluted much earlier and therefore deviated from the calibration curve. For this reason, molecular weight assignment of cTn elution profiles should not be based on the molecular weights of globular proteins. This is especially important for elongated shaped structures like cTnT $[18,19]$. In the present study, we identified that the most abundant form of circulating cTnT detected by the cTnT immunoassay is actually a mixture of cTnT fragments. Previous findings have also postulated that ternary structures of the cTn complex are the most frequent form present in the patient's circulation $[7,16,17,20]$. Using the same gel filtration technique $[16,17]$ we observed that this type of GFC column was not suitable for adequate separation of ternary bound cTn T-I-C complex $\left(\mathrm{V}_{\mathrm{R}}=3 \mathrm{~mL}\right)$ and intact cTnT $\left(\mathrm{V}_{\mathrm{R}}\right.$ $=5 \mathrm{~mL}$ ) molecules. Therefore, the presence of ternary bound cTn molecules in the patient's circulation requires further study.

Furthermore, sera from AMI patients, drawn at standardized time points after admission to the emergency department, and analyzed directly with Western blot analysis revealed that cTnT is degraded in a time-dependent pattern. Intact cTnT was the largest cTnT form that could be detected and it was only present during the early hours ( 4 to 8 hours) after admission in three of the eighteen patients. Primarily $29 \mathrm{kDa}$ cTnT fragments were visible up to 24 hours after AMI, corresponding to the peak concentrations of the cTnT release curve and the period of diagnostic testing. Later on, $29 \mathrm{kDa}$ fragments seemed to diminish and degradation products with a smaller molecular mass ( $\mathrm{MW}_{\mathrm{est}}$ of 14 and $16 \mathrm{kDa}$ ) 
dominated in peripheral blood. The immunoreactivity on the Western blot however seemed to differ between intact and low-molecular weight CTnT molecules, thereby limiting quantification of Western blot bands.

Since, Roche Diagnostics is the only manufacturer of clinical cTnT assays, cTnT degradation does not affect harmonization between different assays, as previously reported for the cTnl assays [11]. To ensure quality of the cTnT measurement in blood samples, Roche Diagnostics recommends the use of recombinant human CTnT spiked in a human serum matrix for monitoring the calibration and precision of the assay. However, as shown in the present study, this recombinant intact $\mathrm{CTnT}$ is not representative for AMI patient serum which contains mainly cTnT fragments. Furthermore, although studies have indicated the magnitude of cTnT measurements to be of prognostic value [21-23] and strongly correlated with infarct size [24, 25], whether the detection of different cTnT forms will affect the clinical utility of cTnT measurements has to be examined.

A limitation to this study is that the 4th generation cTnT assay was used. However, the epitopes of the capture and detection antibody are the same for the current hs-cTnT assay [26]. Subsequently, the same cTnT forms will be detected by both assays. As cTnT fragments were observed using two different techniques and cTnT degradation exhibited a time-dependent pattern, these fragments are unlikely to have been introduced by our methodology, and the time-dependent pattern suggests they are the products of an enzymatic process. Moreover, when the purified cTn T-I-C standard (NIST-SRM 2921) was spiked into serum or heparin plasma, cTnT was shown not to be susceptible to degradation when stored for 72 hours at $4^{\circ} \mathrm{C}$ or for 24 hours at $37^{\circ} \mathrm{C}[27,28]$. It should be noted that the NIST-SRM 2921 standard originally contained the 29 $\mathrm{kDa}$ fragment of $\mathrm{cTnT}$ before incubation. These finding further suggests that the cTnT degradation observed in this study is induced in vivo. In addition, several in vitro studies have demonstrated that the degradation of $\mathrm{cTnT}$ occurred upon ischemia, due to the activity of calpain-I [29-31] and caspase-3 [32] in the intracellular environment of cultured cardiomyocytes, possibly leading to the cleavage of the $\mathrm{N}$ terminus of $\mathrm{cTnT}$. This all leads to the intriguing question of what modification patterns of cTn are present when myocardial necrosis is 
thought to be absent, for instance after physical activity [33, 34], and the subsequent question of whether the diagnostic potential of cTnT can be further improved. Of note, the present study describes cTnT degradation in serum of AMI patients; the presence of cTnT fragments in heparin plasma samples has yet to be investigated.

In conclusion, this study demonstrates that circulating cTnT undergoes degradation in serum following $\mathrm{AMI}$ and the degradation pattern is dependent on time after symptom onset. Importantly, this is the first study to reveal that the cTnT immunoassay widely applied in the clinic binds both intact as degradation products of cTnT during the time frame of diagnostic testing.

\section{Acknowledgement}

We thank Dr. M.T.M. Raijmakers for his kind contribution.

\section{Disclosures}

This study was supported by a research grant from Stichting De Weijerhorst to M.P.v.D.-V. Roche Diagnostics provided the cTnT antibodies for this study. 


\section{References}

1. Thygesen, K., J.S. Alpert, A.S. Jaffe, et al., Third Universal Definition of Myocardial Infarction. Circulation, 2012. 60(16): p. 1581-98.

2. International Federation of Clinical Chemistry and Laboratory Medicine (IFCC) Working group on the Standardization of Troponin I (WG-TNI). Troponin assay analytical characteristics. [cited 2012 June ]; Available from: http://www.ifcc.org/PDF/ScientificActivities/IFCC_Troponin_Table_vDec_2010_FINAL_ng_L _28Jan11.pdf.

3. Collinson, P.O., M.P. van Dieijen-Visser, K. Pulkki, et al., Evidence-based laboratory medicine: how well do laboratories follow recommendations and guidelines? The Cardiac Marker Guideline Uptake in Europe (CARMAGUE) study. Clin Chem, 2012. 58(1): p. 305-6.

4. Katus, H.A., A. Remppis, T. Scheffold, et al., Intracellular compartmentation of cardiac troponin $\mathrm{T}$ and its release kinetics in patients with reperfused and nonreperfused myocardial infarction. Am J Cardiol, 1991. 67: p. 1360-7.

5. Bleier, J., K.P. Vorderwinkler, J. Falkensammer, et al., Different intracellular compartmentations of cardiac troponins and myosin heavy chains: a causal connection to their different early release after myocardial damage. Clin Chem, 1998. 44(9): p. 1912-8.

6. Jeong, E.M., X. Wang, K. Xu, et al., Nonmyofilament-associated troponin T fragments induce apoptosis. Am J Physiol Heart Circ Physiol, 2009. 297(1): p. H283-92.

7. Wu, A.H., Y.J. Feng, R. Moore, et al., Characterization of cardiac troponin subunit release into serum after acute myocardial infarction and comparison of assays for troponin $\mathrm{T}$ and $\mathrm{I}$. American Association for Clinical Chemistry Subcommittee on cTnl Standardization. Clin Chem, 1998. 44(6 Pt 1): p. 1198-208.

8. Labugger, R., L. Organ, C. Collier, et al., Extensive troponin I and T modification detected in serum from patients with acute myocardial infarction. Circulation, 2000. 102(11): p. 1221-6.

9. McDonough, J.L., D.K. Arrell, and J.E. Van Eyk, Troponin I degradation and covalent complex formation accompanies myocardial ischemia/reperfusion injury. Circ Res, 1999. 84(1): p. 920.

10. Madsen, L.H., G. Christensen, T. Lund, et al., Time course of degradation of cardiac troponin $\mathrm{I}$ in patients with acute ST-elevation myocardial infarction. The ASSENT-2 troponin substudy. Circ Res, 2006. 99(10): p. 1141-7.

11. Katrukha, A.G., A.V. Bereznikova, V.L. Filatov, et al., Degradation of cardiac troponin I: implication for reliable immunodetection. Clin Chem, 1998. 44(12): p. 2433-40.

12. Narolska, N.A., N. Piroddi, A. Belus, et al., Impaired diastolic function after exchange of endogenous troponin I with C-terminal truncated troponin I in human cardiac muscle. Circ Res, 2006. 99(9): p. 1012-20.

13. Galinska, A., V. Hatch, R. Craig, et al., The C terminus of cardiac troponin I stabilizes the Ca2+-activated state of tropomyosin on actin filaments. Circ Res, 2010. 106(4): p. 705-11.

14. Diris, J.H., C.M. Hackeng, J.P. Kooman, et al., Impaired Renal Clearance Explains Elevated Troponin T Fragments in Hemodialysis Patients. Circulation, 2004. 109(1): p. 23-5.

15. Michielsen, E.C., J.H. Diris, V.W. Kleijnen, et al., Investigation of release and degradation of cardiac troponin T in patients with acute myocardial infarction. Clin Biochem, 2007. 40(12): p. 851-5.

16. Bates, K.J., E.M. Hall, M.N. Fahie-Wilson, et al., Circulating immunoreactive cardiac troponin forms determined by gel filtration chromatography after acute myocardial infarction. Clin Chem, 2010. 56(6): p. 952-958.

17. Fahie-Wilson, M.N., D.J. Carmichael, M.P. Delaney, et al., Cardiac troponin T circulates in the free, intact form in patients with kidney failure. Clin Chem, 2006. 52(3): p. 414-420.

18. Michielsen, E.C., J.H. Diris, V.W.V.C. Kleijnen, et al., Size-exclusion chromatography of circulating cardiac troponin T. Clin Chem, 2006. 52(12): p. 2306-7.

19. Michielsen, E.C., J.H. Diris, V.W.V.C. Kleijnen, et al., Interpretation of cardiac troponin T behaviour in size-exclusion chromatography. Clin Chem Lab Med, 2006. 44(12): p. 1422-7. 
20. Katrukha, A.G., A.V. Bereznikova, T.V. Esakova, et al., Troponin I is released in bloodstream of patients with acute myocardial infarction not in free form but as complex. Clin Chem, 1997. 43(8 Pt 1): p. 1379-85.

21. Hamm, C.W., J. Ravkilde, W. Gerhardt, et al., The prognostic value of serum troponin T in unstable angina. N Engl J Med, 1992. 327(3): p. 146-50.

22. Latini, R., S. Masson, I.S. Anand, et al., Prognostic value of very low plasma concentrations of troponin $\mathrm{T}$ in patients with stable chronic heart failure. Circulation, 2007. 116(11): p. 1242-9.

23. Stubbs, P., P. Collinson, D. Moseley, et al., Prognostic significance of admission troponin T concentrations in patients with myocardial infarction. Circulation, 1996. 94(6): p. 1291-7.

24. Giannitsis, E., H. Steen, K. Kurz, et al., Cardiac magnetic resonance imaging study for quantification of infarct size comparing directly serial versus single time-point measurements of cardiac troponin T. J Am Coll Cardiol, 2008. 51(3): p. 307-14.

25. Steen, H., E. Giannitsis, S. Futterer, et al., Cardiac troponin T at 96 hours after acute myocardial infarction correlates with infarct size and cardiac function. J Am Coll Cardiol, 2006. 48(11): p. 2192-4.

26. Giannitsis, E., K. Kurz, K. Hallermayer, et al., Analytical validation of a high-sensitivity cardiac troponin T assay. Clin Chem, 2010. 56(2): p. 254-61.

27. Cobbaert, C.M., C.W. Weykamp, E.C. Michielsen, et al., Time-dependent instability of cardiac troponins in human plasma spiked with NIST reference material 2921. Clin Chem, 2008. 54(12): p. 2078-9.

28. Mingels, A., C. Cobbaert, N. Smit, et al., Time- and temperature-dependent stability of NIST standard reference material 2921 in serum and plasma. Clin Chem Lab Med 2012. [In press].

29. Ke, L., X.Y. Qi, A.J. Dijkhuis, et al., Calpain mediates cardiac troponin degradation and contractile dysfunction in atrial fibrillation. J Mol Cell Cardiol, 2008. 45(5): p. 685-93.

30. Feng, H.Z., B.J. Biesiadecki, Z.B. Yu, et al., Restricted N-terminal truncation of cardiac troponin T: A novel mechanism for functional adaptation to energetic crisis. J Physiol, 2008. 283(48): p. 33384-33393.

31. Zhang, Z., B.J. Biesiadecki, and J.P. Jin, Selective deletion of the NH2-terminal variable region of cardiac troponin $\mathrm{T}$ in ischemia reperfusion by myofibril-associated mu-calpain cleavage. Biochemistry, 2006. 45(38): p. 11681-94.

32. Communal, C., M. Sumandea, P. de Tombe, et al., Functional consequences of caspase activation in cardiac myocytes. Proc Natl Acad Sci U S A, 2002. 99(9): p. 6252-6.

33. Mingels, A., L. Jacobs, E. Michielsen, et al., Reference population and marathon runner sera assessed by highly sensitive cardiac troponin $\mathrm{T}$ and commercial cardiac troponin $\mathrm{T}$ and $\mathrm{I}$ assays. Clin Chem, 2009. 55(1): p. 101-8.

34. Middleton, N., K. George, G. Whyte, et al., Cardiac troponin T release is stimulated by endurance exercise in healthy humans. J Am Coll Cardiol, 2008. 52(22): p. 1813-4. 


\section{Optimization of elution procedure}

A

$56^{\circ} \mathrm{C}$

$1 \mathrm{M}$ glycine $\mathrm{pH} 3$

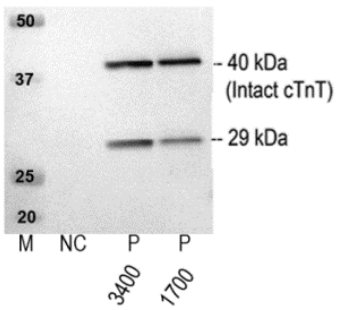

B
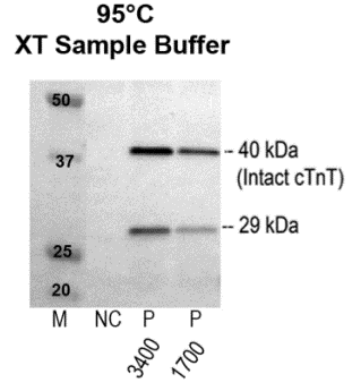

C
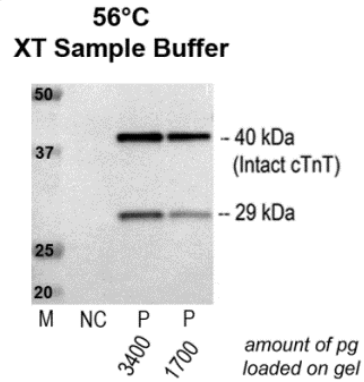

SUPPLEMENTAL FIGURE 1. Optimization of the elution procedure during immunoprecipitation. Troponin T-IC complex spiked in CTnT-negative serum was immunoprecipitated in triplo according to the methods described in the manuscript. Three different elution conditions were tested: (A) $1 \mathrm{M}$ glycine $\mathrm{pH} 3$ at $56^{\circ} \mathrm{C}$ (B) XT sample buffer (BioRad) at $95^{\circ} \mathrm{C}$ and (C) at $56^{\circ} \mathrm{C}$.

A

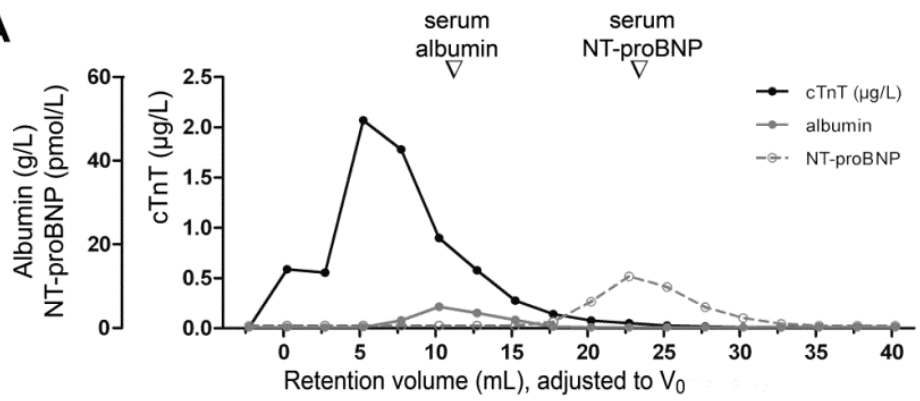

B

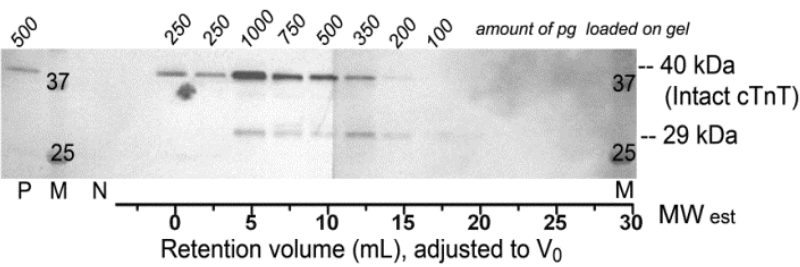

SUPPLEMENTAL FIGURE 2. GFC analysis of purified CTnT (A) Chromatographic curve for purified free cTnT spiked in cTn-negative serum; serum albumin $\left(\mathrm{V}_{R}=11 \mathrm{~mL}\right)$ and serum NT-proBNP $\left(\mathrm{V}_{R}=22 \mathrm{~mL}\right)$ were used as internal standards. (B) Purification and detection of cTnT by means of the cTnT antibodies of the clinical assay in the obtained chromatographic fractions. $\mathrm{P}$, positive control; $\mathrm{M}$, marker; $\mathrm{N}$, negative control. 


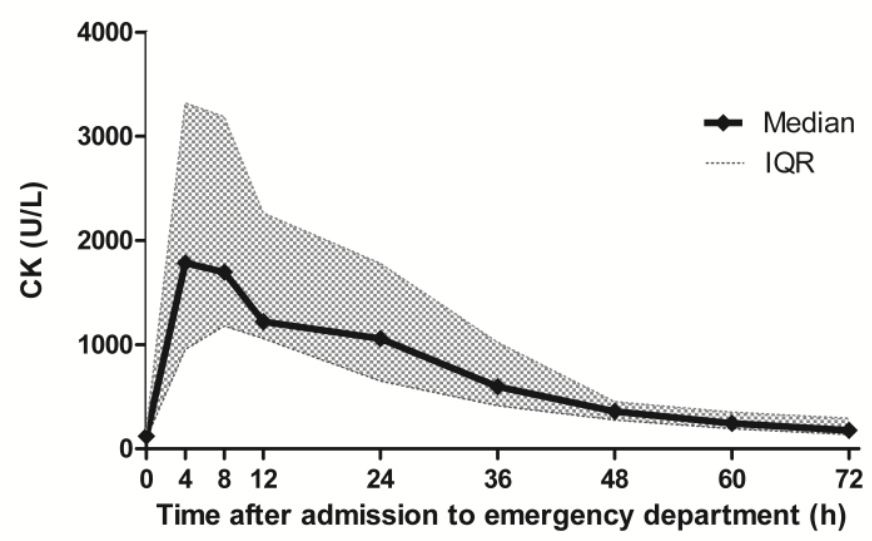

SUPPLEMENTAL FIGURE 3. Release kinetics of creatine kinase (CK) after AMI ( $n=18)$, represented by the median (black) and IQR (grey) concentrations of CK. U/L indicates units per liter.

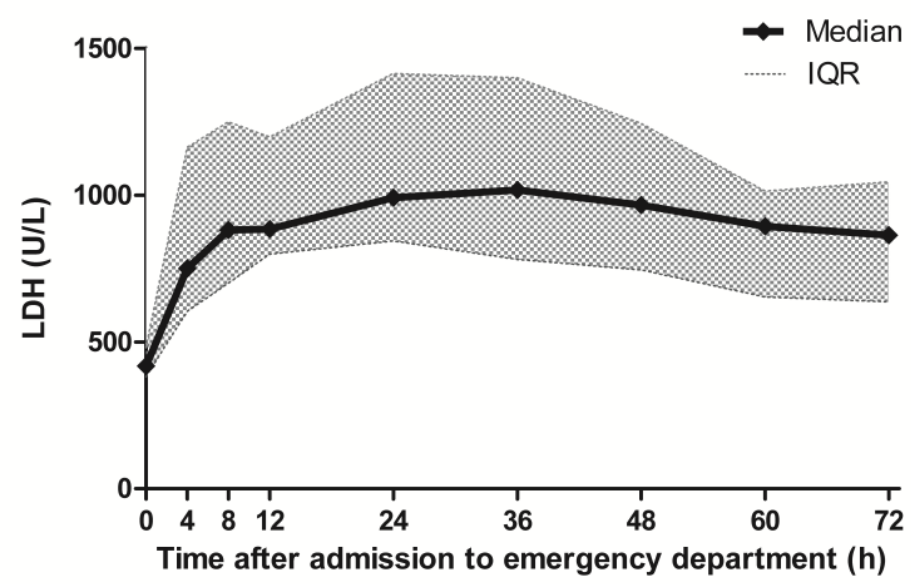

SUPPLEMENTAL FIGURE 4. Release kinetics of lactate dehydrogenase (LD) after AMI ( $n=18)$, represented by the median (black) and IQR (grey) concentrations of LD. U/L indicates units per liter. 
A

Roche detection antibody directed against middle region of cTnT (a.a.r 125-131)

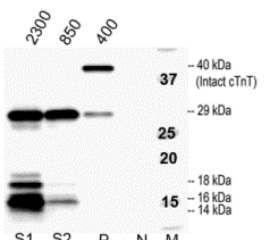

B Detection antibody directed
against the $\mathbf{N}$ terminus of cTnT (a.a.r. 1-60)

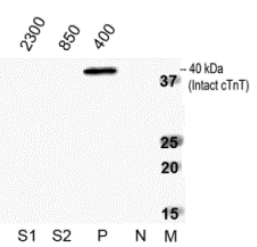

C

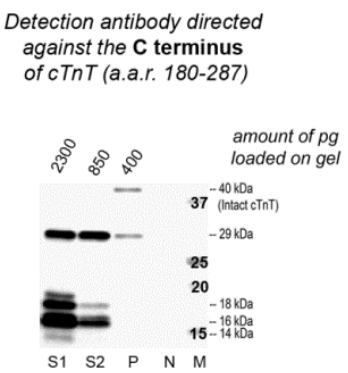

SUPPLEMENTAL FIGURE 5. Western blot analysis using different CTnT antibodies. Two serum samples (S1 and S2) immunoprecipitated in triplo with and loaded on the SDS-PAGE gel according to the specifications given in the methods section. Each Western blot was subsequently stained with different cTnT antibodies, namely (A) Roche detection antibody (M7); (B) antibody directed against $\mathrm{N}$ terminus of cTnT (1 $\mu \mathrm{g} / \mathrm{mL}, 9 \mathrm{G6}$, Hytest Ltd) and (C) the $C$ terminus of $c \operatorname{TnT}(1 \mu \mathrm{g} / \mathrm{mL}, 7 A 9$, Hytest). The secondary antibody was for all Western blots goat-anti mouse peroxidase $(0.4 \mu \mathrm{g} / \mathrm{mL}$; DAKO). S1 indicates serum sample of patient 1 collected 12 hours after admission, [cTnT] = $14 \mu \mathrm{g} / \mathrm{L}$; S2, serum sample of patient 2 collected 8 hours after admission, $[\mathrm{cTnT}]=4.3 \mu \mathrm{g} / \mathrm{L} ; \mathrm{P}$, positive control (troponin T-I-C complex spiked in CTnT-negative serum, $[\mathrm{cTnT}]=2 \mu \mathrm{g} / \mathrm{L}$ ); N, negative control (= cTnT-negative serum; [cTnT]<0.01 $\mu \mathrm{g} / \mathrm{L}$ )

SUPPLEMENTAL TABLE 1. Recoveries (\%) of cTnT and cTnl in gel filtration chromatography

\begin{tabular}{|c|c|c|}
\hline Recovery (\%) $^{\text {a }}$ & cTnT & cTnl \\
\hline Troponin T-I-C & $96 \%$ & $79 \%$ \\
\hline Purified cTnT & $89 \%$ & - \\
\hline Purified cTnl & - & $79 \%$ \\
\hline AMl patients & $77 \%$ b & $99 \%{ }^{\mathbf{c}}$ \\
\hline
\end{tabular}

${ }^{\mathrm{a}}$, all recoveries were calculated as follows:

$$
\text { recovery }(\%)=\frac{\sum\left(\left(c \operatorname{Tn} T \text { concentration }\left(\frac{p g}{m L}\right)\right)_{\text {fractions }} \times \text { fraction volume }(1.25 \mathrm{~mL})\right)}{\left(\left(c \operatorname{TnT} \text { concentration }\left(\frac{p g}{m L}\right)\right)_{\text {initial }} \times \text { injection volume }(1 \mathrm{~mL})\right)} \times 100
$$

${ }^{\mathrm{b}}$, mean recovery of all 24 GFC analyses; ${ }^{c}$, mean recovery based on 12 of the 24 GFC analyses 
SUPPLEMENTAL TABLE 2. Baseline characteristics of AMI patients $(n=18)$ in which the molecular forms of cTnT were investigated at standardized time-points (0-72 hours after hospital admission).

\begin{tabular}{|c|c|c|c|c|c|c|}
\hline $\mathrm{N}^{\circ}$ & Sex & $\begin{array}{l}\text { Age } \\
\text { (yrs) }\end{array}$ & Lesion $^{\mathrm{a}}$ & $\begin{array}{l}\Delta \text { time symptom onset } \\
\text { and admission } \\
\text { (h:min) }\end{array}$ & $\begin{array}{c}\text { ST max } \\
(\mathrm{mV})^{\mathrm{c}}\end{array}$ & $\begin{array}{l}\text { MRI } \\
(\%)^{d}\end{array}$ \\
\hline 1 & $F$ & 66 & RCA prox & $4: 07$ & -3.8 & 18.3 \\
\hline 2 & M & 56 & RCA dist & $2: 35$ & 6.9 & 27.6 \\
\hline 3 & M & 78 & RCA mid & $2: 42$ & 7.9 & n.a. \\
\hline 4 & $M$ & 58 & RCA prox & $1: 55$ & 7.1 & 25.8 \\
\hline 5 & M & 52 & RCA mid & $2: 17$ & 2.8 & 22.0 \\
\hline 6 & $\mathrm{~F}$ & 70 & RCA prox & $5: 30$ & 5.1 & 10.5 \\
\hline 7 & $F$ & 50 & LAD mid & 4:09 & 4.9 & 23.2 \\
\hline 8 & $M$ & 55 & LAD prox & $2: 12$ & 9.6 & 36.9 \\
\hline 9 & $M$ & 58 & LAD mid & $5: 30$ & 5.4 & 0.8 \\
\hline 10 & $F$ & 74 & LAD mid & $2: 12$ & n.a. & 24.5 \\
\hline 11 & $F$ & 66 & RCA mid & $2: 00$ & 2.0 & 5.0 \\
\hline 12 & $M$ & 54 & RCA prox & $3: 30$ & 3.3 & 10.3 \\
\hline 13 & $M$ & 64 & LAD prox & $3: 45$ & 14.4 & n.a. \\
\hline 14 & M & 66 & RCA prox & $3: 15$ & 7.6 & 25.4 \\
\hline 15 & $\mathrm{~F}$ & 48 & LAD mid & 4:00 & 8.8 & n.a. \\
\hline 16 & M & 44 & RCA prox & $4: 30$ & n.a. & 19.9 \\
\hline 17 & $M$ & 61 & RCA mid & $2: 15$ & 6.4 & 21.3 \\
\hline 18 & $M$ & 72 & RCA prox & $0: 45$ & -1.9 & 2.5 \\
\hline
\end{tabular}

a, indicates coronary artery in which stent was placed; b, time between symptom onset and hospital admission (hours:minutes), c, the maximum ST segment elevation during Holter electrocardiography; d, infarct size percentage measured with late gadolinium enhancement MRI; $F$, indicates female; $M$, male; RCA, right coronary artery; LAD, left anterior descending artery; $\mathrm{mV}$, millivolt; $\mathrm{U}$, units; $n$.a., not available 



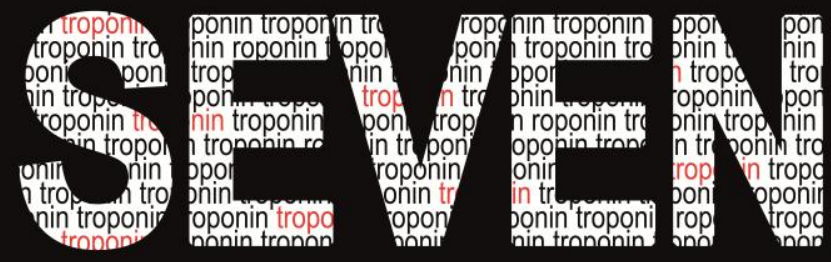

\section{ACCUMULATION OF N-TERMINAL TRUNCATED CARDIAC TROPONIN T FRAGMENTS IN PATIENTS WITH END-STAGE RENAL DISEASE ON HEMODIALYSIS}

This project was funded with the Noyons Stipendium 2014 research grant awarded to E. Cardinaels and A. Mingels 


\section{Abstract}

\section{Background}

Cardiac troponin $\mathrm{T}$ (cTnT) is known to be chronically elevated in patients with end-stage renal disease (ESRD), which is attributed to the presence of cardiac disease but also renal dysfunction. To date it remains unknown whether these chronically elevated cTnT concentrations reflect the accumulation of degraded or intact cTnT molecules.

\section{Methods}

By gel filtration chromatography (GFC), retention volumes for purified standards (cTnT-I-C complex and intact CTnT) and the clinical assay's quality control materials were investigated. Additionally, GFC profiles of acute myocardial infarction (AMI) patients' sera were determined. Eluates were subjected to immunoprecipitation and Western blotting, employing antibodies from the Roche immunoassay to mimic the clinical cTnT assay. Also, AMI patients sera were subjected directly to immunoblotting using either the detector antibody of the clinical assay or an antibody directed against the N-terminal end of cTnT. The validated GFC analysis was then applied to the sera from ESRD patients before and after hemodialysis $(n=10)$ and over 2 months follow-up $(n=6)$ to establish the molecular forms of cTnT.

\section{Results}

Retention volumes $\left(\mathrm{V}_{\mathrm{R}}\right)$ of cTn T-I-C complex and intact cTnT were found to be 20 and $27.5 \mathrm{~mL}$, respectively. Also in quality control materials, cTnT eluted at $27.5 \mathrm{~mL}$. AMI patients' sera revealed two cTnT peaks with retention volumes of 27.5 and $45 \mathrm{~mL}$, and Western blotting illustrated that these peaks corresponded to N-terminal truncated cTnT fragments of 29 and $<18 \mathrm{kDa}$, respectively. In contrast to AMI, ESRD patients demonstrated only one reproducible cTnT-peak at $45 \mathrm{~mL}$ in serum samples obtained before as well as after hemodialysis. Moreover, this cTnT-peak remained stable following 2 months in time. This cTnT-peak could be allocated to the low-molecular weight cTnT fragments $(<18$ $\mathrm{kDa}$ ) in which the N-terminal end was found to be absent.

\section{Conclusion}

This is the first study to clearly demonstrate the presence of only degraded cTnT forms in the ESRD patients' sera. Moreover, these cTnT-fragments were observed before and after hemodialysis, and were found to be stable over time. 


\section{Introduction}

The release of cardiac troponins (cTn) in the patient's circulation is considered to be a hallmark for myocardial damage because of their unique cardiospecificity. The diagnosis of an acute myocardial infarction (AMI) therefore relies on an increase or decrease in serial cTn measurements, preferably performed with high-sensitvitiy cTn (hs-cTn) assays [1]. Previously, we have demonstrated in serial AMI patients' sera that cTnT is degraded in a time-dependent pattern [2]. During the first hours after presentation at emergency department (ED), predominantly $29 \mathrm{kDa}$ cTnT-fragments were present, while later on (>24 h after ED-presentation) only fragments <18 kDa were detected.

Chronically elevated cTnT concentrations are also common in patients suffering from renal dysfunction [3-5]. The presence of measurable cTnT concentrations in these patients have been associated with abnormal left ventricular morphology and function [6], myocardial stunning [7], and with a higher risk on future cardiovascular events and mortality [8, 9]. Apart from this, significant associations between cTnT and renal clearance have been demonstrated [10]. It has been shown that these chronically elevated cTn concentrations reflect the accumulation of cTnT-fragments, as illustrated with Western blotting employing different antibodies than those of the clinical cTnT assay [11]. However, these findings have been opposed by others, who found only intact cTnT when analyzing the serum of ESRD patients with gel filtration chromatography (GFC) [12]. To date it is therefore contradictory which cTnT forms circulate in the serum of ESRD patients and which are detected by the clinical (Roche) cTnT assay. Gaining insight into the molecular forms of cTnT following ESRD, will further improve our understanding on the nature of chronic versus acute cTnT elevations.

In this study, we therefore aim to elucidate the cTnT forms that are detected in the circulation of patients on hemodialysis using the clinical antibodies in a similar approach as previously performed in AMI patients [2], and as described in chapter 6 of this thesis. 


\section{Materials and Methods}

\section{Study populations}

Molecular forms of circulating cTnT were assessed in the serum samples of two ESRD-populations, both on chronic hemodialysis therapy. In a first ESRD cohort of 10 patients, serum samples were drawn before and immediately after hemodialysis. In a second cohort of 6 patients, pre-dialysis serum samples were collected at baseline and 2 months later. All samples were immediately frozen at $-80^{\circ} \mathrm{C}$ upon analysis. Both studies were conducted according to the principles of the Declaration of Helsinki and approved by the local ethics committees. Informed consent was obtained from all patients.

\section{Biochemical testing}

cTnT-concentrations were determined using the hs-cTnT assay (Roche Diagnostics) with a limit of detection at $5 \mathrm{ng} / \mathrm{L}$ and $10 \% \mathrm{CV}$ cutoff at $13 \mathrm{ng} / \mathrm{L}$. NT-proBNP was measured with a limit of detection at $5.08 \mathrm{ng} / \mathrm{L}(0.6 \mathrm{pmol} / \mathrm{L})$ and $\mathrm{CV} 6.8 \%$ at $74.4 \mathrm{ng} / \mathrm{L}$ (8.78 pmol/L). Albumin was analyzed using the microalbumin assay with measuring range of $2.0-970 \mathrm{mg} / \mathrm{L}$. All measurements were performed on the COBAS 6000 analyzer (Roche).

\section{Fractionation by GFC}

GFC was performed on the AKTA Prime Plus (GE Healthcare) equipped with a $1.6 \times 60 \mathrm{~cm}$ Sephacryl-S300 column (GE Healthcare). The column was equilibrated with $0.26 \mathrm{~mol} / \mathrm{L} \mathrm{NaCl}, 2.5 \mathrm{mmol} / \mathrm{L} \mathrm{CaCl}_{2} \cdot 2 \mathrm{H}_{2} \mathrm{O}, 0.02 \mathrm{~mol} / \mathrm{L}$ Tris, 6 $\mathrm{mmol} / \mathrm{L} \mathrm{NaN} \mathrm{Na}_{3}$ and $1 \mathrm{~g} / \mathrm{L}$ bovine serum albumin buffer, $\mathrm{pH} 7.4$ [13] and operated at $0.5 \mathrm{~mL} / \mathrm{min}$. The void volume $\left(\mathrm{V}_{0}\right)$ determination and calibration was conducted using the Gel Filtration Calibration kit (GE Healthcare) containing Dextran Blue (molecular weight (MW), 2000 kDa), conalbumin (MW, 75.0 kDa), ovalbumin (MW, $43.0 \mathrm{kDa}$ ), carbonic anhydrase (MW, $29.0 \mathrm{kDa}$ ) and ribonuclease (MW, $13.7 \mathrm{kDa})$. Dextran Blue $(1 \mathrm{mg} / \mathrm{ml})$ and globular protein standards $(3-4 \mathrm{mg} / \mathrm{ml})$ were dissolved in running buffer, $0.5 \mathrm{~mL}$ was loaded on the column, and absorbance was detected at $280 \mathrm{~nm}$, all in duplo. $\mathrm{V}_{0}$ was determined to be $40 \mathrm{~mL}$. 
For GFC analysis, purified human cTn T-I-C complex (MW, 77 kDa; Hytest) and purified free cTnT (MW, $37 \mathrm{kDa}$, respectively; Hytest) were diluted in running buffer or spiked in serum pool of healthy volunteers (cTnT<14 ng/L). Calibration (level-2) and control (TN2) materials of the clinical Roche hs-cTnT assay were dissolved as prescribed by the manufacturer and immediately examined using GFC. Also residual serum of acute myocardial infarction patients were analyzed to validate previous results [2].

To obtain the standard calibration curve, the gel-phase distribution coefficient $\left(\mathrm{K}_{\mathrm{av}}=\mathrm{V}_{\mathrm{R}} /\left(\mathrm{V}_{\mathrm{c}}-\mathrm{V}_{0}\right)\right)$ was plotted against the logarithm of $\mathrm{MW}$ of globular proteins and purified cTn standards following the equation $y=-0.34 x+1.04$. $V_{R}$ signifies the retention volume adjusted to void volume, while $V_{c}$ indicated column volume and corresponded to $120 \mathrm{~mL}$.

For serum samples, 1.0-2.0 mL was loaded on the column. Serum albumin (67 $\mathrm{kDa}$ ) and serum NT-proBNP were used as internal standards in the patients' sera. For each sample loaded, fractions of $1.25 \mathrm{~mL}$ were collected, kept on ice until immunoassay measurements (within 1 hour) and subsequently stored at $80^{\circ} \mathrm{C}$.

\section{Purification and Western blot detection of cTnT}

Purification and characterization of cTnT was performed using the antibodies from the Roche cTnT-assay (as kindly provided by Roche Diagnostics, www.roche.com). The capture cTnT antibody (M11.7, epitope a.a.r. 136-147) was used for immunoprecipitation of cTnT. To each $\mathrm{mL}$ of magnetic streptavidin-coated dynabeads (Invitrogen) $10 \mu \mathrm{g}$ biotinylated M11.7 was added. Additionally, beads were crosslinked three times with dimethyl pimelimidate. Subsequently, $200-700 \mu \mathrm{L}$ of serum was precipitated with $50 \mu \mathrm{L}$ of M11.7-coated beads for 1 hour at $4^{\circ} \mathrm{C}$. The average cTnT yield after immunoprecipitation was $>90 \%$ (data not shown). After being washed with PBS containing $0.1 \%$ Tween-20 (PBST) the immunoprecipitates were eluted with $1 \mathrm{M}$ glycine $(18 \mu \mathrm{L}), \mathrm{pH} 3$ for $15 \mathrm{~min}$ at $56^{\circ} \mathrm{C}$.

As negative controls for immunoblotting of GFC samples and AMI patients' sera, running buffer or the serum pool of healthy volunteers was used, respectively. As positive controls, purified intact cTnT was spiked in running buffer and cTnT 
negative pooled serum, respectively. Only GFC fractions containing sufficiently high cTnT concentrations ( $500 \mathrm{ng} / \mathrm{L}$ ) could be visualized on the immunoblot. Immunoprecipitates were diluted with XT Sample Buffer $(6 \mu \mathrm{L})$ (Bio-Rad Laboratories), reduced at $56^{\circ} \mathrm{C}$, and separated on a $12 \%$ Criterion XT SDSPAGE gel (Bio-Rad) together with the Precision Plus Protein Standard (BioRad). After stacking for $15 \mathrm{~min}$ at $100 \mathrm{~V}$ the proteins were resolved for $45 \mathrm{~min}$ at $200 \mathrm{~V}$ in XT MOPS electrophoresis buffer (Bio-Rad) and were transferred to the nitrocellulose membrane (Bio-Rad, $0.45 \mu \mathrm{m}$ ) at $100 \mathrm{~V}$ for $1 \mathrm{~h}$ at $4^{\circ} \mathrm{C}$. The membranes were treated with the vacuum SNAP i.d. protein detection system (Millipore) according to manufacturer instructions. Non-specific binding was blocked with $0.5 \%$ non-fat dry milk Blocking-Grade Blocker (Bio-Rad) in PBST. Primary antibodies were Roche M7 anti-cTnT (epitope a.a.r. 125-131; $10 \mu \mathrm{g} / \mathrm{L}$ ), and cTnT-specific antibodies against the middle region (Hytest, 1C11, epitope a.a.r. 95-181; $7 \mu \mathrm{g} / \mathrm{L}$ ) and the $\mathrm{N}$-terminal region (Hytest, 9G6; epitope a.a.r. 1$60 ; 1 \mu \mathrm{g} / \mathrm{L})$. As the secondary antibody, goat anti-mouse peroxidase $(0.4 \mu \mathrm{g} / \mathrm{mL}$; DAKO) diluted in PBST were employed. Subsequently, membranes were incubated in Super Signal West Femto Substrate (Thermo Scientific) and bands were detected using the ChemiDoc XRS scanner (Bio-Rad) and Quantity One Software (Bio-Rad, Version 4.6.5.).

\section{Results}

\section{GFC analysis of control and calibration material for clinical assay}

GFC-analysis of purified cTn standards (cTn T-I-C complex, intact cTnT) spiked in cTnT-negative serum revealed adjusted retention volumes $\left(V_{R}\right)$ of 20 and $27.5 \mathrm{~mL}$, respectively (FIGURE $1 \mathrm{~A}$ and $\mathrm{B}$ ). Both standards eluted before the internal standard serum albumin $\left(\mathrm{V}_{\mathrm{R}}=32.5 \mathrm{~mL}\right)$ and NTproBNP $\left(\mathrm{V}_{\mathrm{R}}=45 \mathrm{~mL}\right)$. Western blotting of these GFC fractions using the clinical cTnT antibodies, confirmed that these two peaks both contain intact cTnT forms (estimated MW, $\mathrm{MW}_{\text {est }}$ of $40 \mathrm{kDa}$; data not shown) as previously described [2].

Furthermore, GFC-elution profiles of the control (TN-2; cTnT = $1921 \mathrm{ng} / \mathrm{L})$ and calibrator (level 2; cTnT $=5586 \mathrm{ng} / \mathrm{L}$ ) material of the clinical hs-cTnT assay both revealed a cTnT-peak at $27.5 \mathrm{~mL}$ (FIGURE $1 \mathrm{C}$ and $\mathrm{D}$ respectively). 


\section{A. cTn-T-I-C complex in cTn-negative serum}

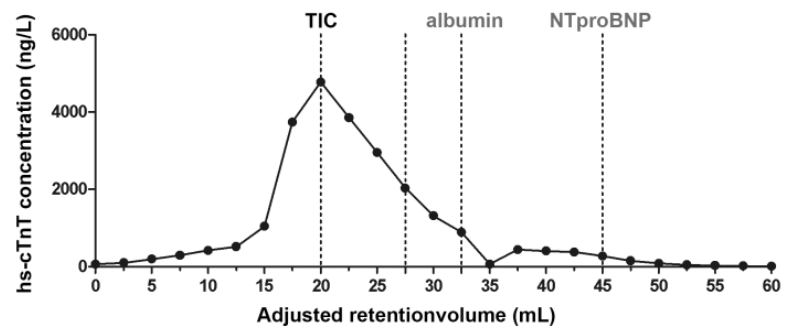

\section{B. intact cTnT in cTn-negative serum}

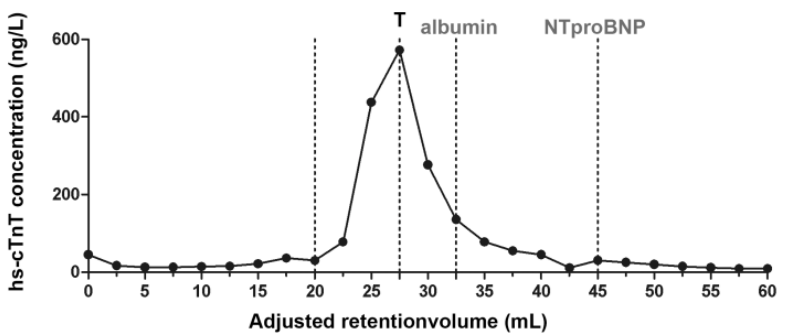

\section{Control TN2 (Roche Diagnostics)}

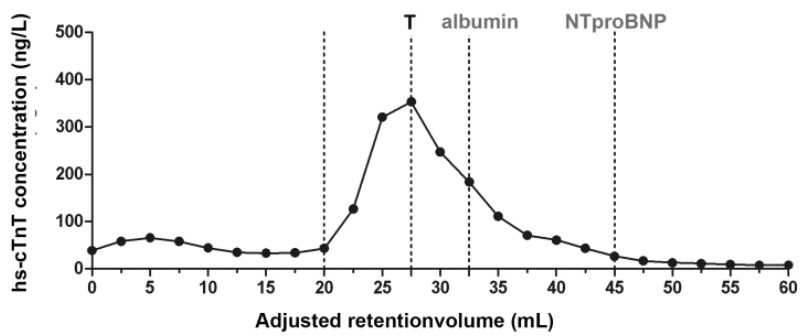

\section{Calibrator L 2 (Roche Diagnostics)}

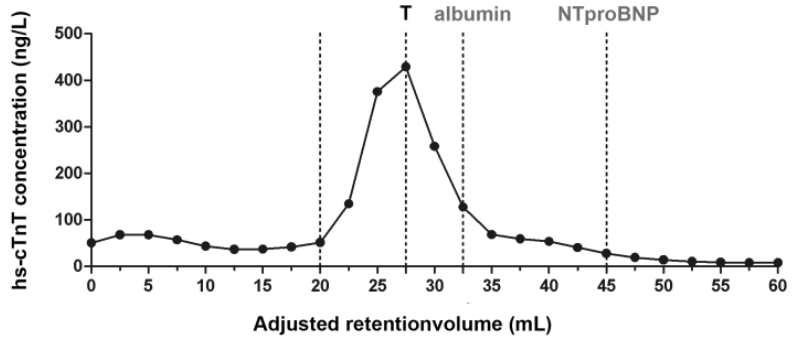

FIGURE 1. cTnT-peak profiles obtained by GFC analysis of purified cTn-T-I-C complex (A), purified intact cTnT (B), Roche clinical assay control (C) and calibration (D) material. In grey, retention volumes of the internal serum standards are indicated. 


\section{Validation of N-terminal cTnT fragments in AMI patients' sera.}

As depicted in FIGURE 2A, the two-peak elution profiles of residual AMI serum samples was validated as previously described [2]. Retention volumes of 27.5 and $45 \mathrm{~mL}$ were established, eluting before and after the internal standard serum albumin $\left(\mathrm{V}_{\mathrm{R}}=32.5 \mathrm{~mL}\right)$, respectively. Western blot analysis assigned the first cTnT peak $\left(\mathrm{V}_{\mathrm{R}}\right.$ of $\left.27.5 \mathrm{~mL}\right)$ as the $29 \mathrm{kDa}\left(\mathrm{MW}_{\text {est }}\right)$-degradation product of cTnT (FIGURE 2A, right panel). The second peak $\left(\mathrm{V}_{\mathrm{R}}=45 \mathrm{~mL}\right)$ was allocated to even smaller cTnT degradation products of $\mathrm{MW}_{\text {est }}$ between 14 and $18 \mathrm{kDa}$.

A. Gel Filtration Chromatography

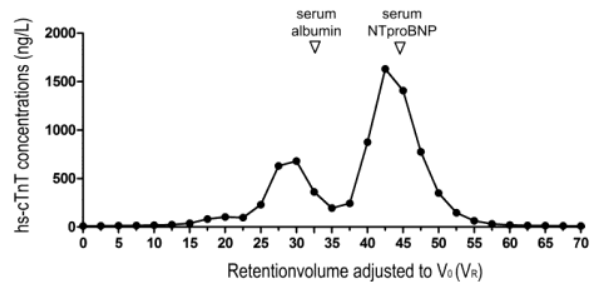

B. Western Blotting

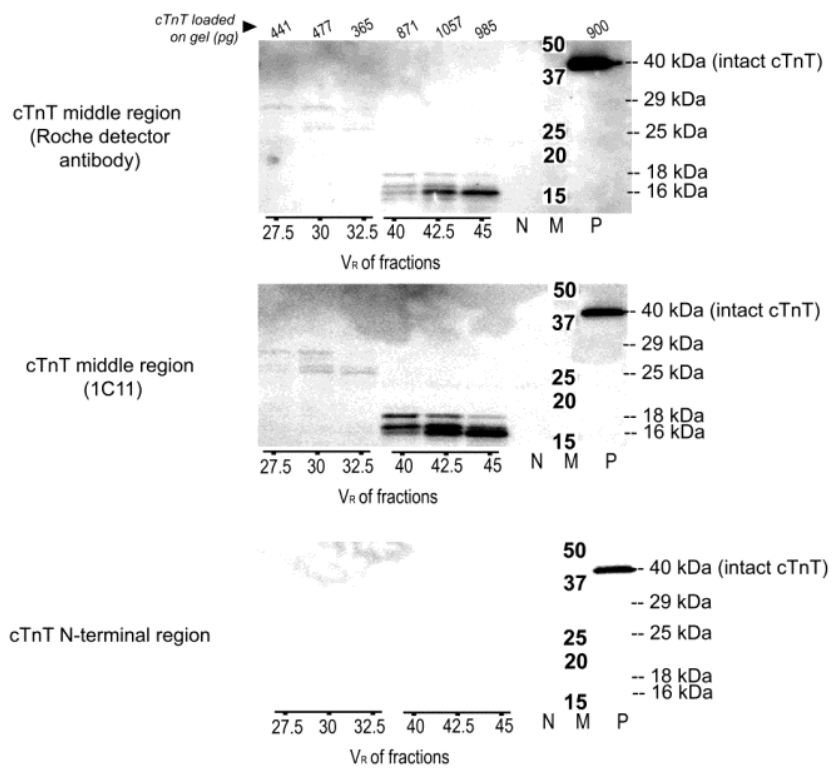

FIGURE 2. GFC profile of a representative AMI patient (A), followed by Western blot analysis using the Roche cTnT antibodies (B), 1C11 antibodies for the detection of the middle region of $\mathrm{CTnT}$ (C) and an antibody directed against the $\mathrm{N}$-terminal end of CTnT (D). P, indicates positive control of intact CTnT spiked GFC buffer; $\mathrm{M}$, marker and $\mathrm{N}$, negative control. 
Furthermore, direct immunoprecipitation of residual AMI sera followed by Western blotting using the clinical cTnT antibodies assay (Roche M7 antibody) validated the presence of these $29 \mathrm{kDa}$ and $<18 \mathrm{kDa}$ cTnT-fragments that were also observed in the first and second GFC peak respectively (SUPPLEMENTAL FIGURE 1, left panel). However, none of these fragments demonstrated immunoreactivity with the antibody directed against the $\mathrm{N}$-terminal end of CTnT (SUPPLEMENTAL FIGURE 1, right panel).
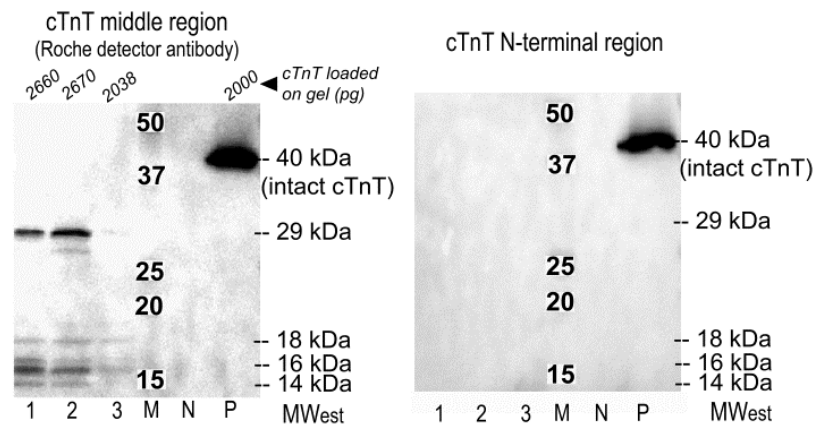

SUPPLEMENTAL FIGURE 1. Western blot analysis of AMI patients' serum samples using either Roche M7 (left) or the N-terminal CTnT (right) detection antibody. 1-3, indicates serum samples of $3 \mathrm{AMI}$ patients; $\mathrm{P}$, positive control containing intact cTnT spiked in serum; $\mathrm{M}$, marker and $\mathrm{N}$, negative control.

\section{Presence and stability of $\mathrm{N}$-terminal truncated cTnT fragments in ESRD patients}

In the first ESRD population ( $\mathrm{N}=10)$, median (IQR) hs-cTnT concentrations were 68.1 (52.2-80.4) $\mathrm{ng} / \mathrm{L}$ and 62.5 (43.5-79.0) $\mathrm{ng} / \mathrm{L}$ pre- versus post-dialysis, respectively (SUPPLEMENTAL FIGURE 2), demonstrating a minor and nonsignificant reduction in hs-cTnT concentrations following hemodialysis. 


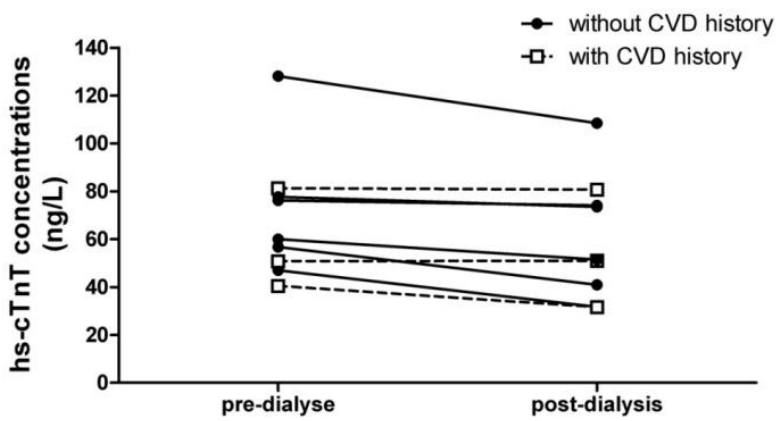

SUPPLEMENTAL FIGURE 2. Hs-cTnT concentrations measured in serum of ESRD patients $(n=10)$ before and after hemodialysis

GFC-elution profiles of these serum samples indicated a reproducible peak at adjusted $\mathrm{V}_{\mathrm{R}}$ of $45 \mathrm{~mL}$ before and after conventional hemodialysis (Figure $3 A$ and B). Also in the GFC analysis, a non-significant reduction in cTnT-peak was observed post-hemodialysis.

In the second ESRD population $(\mathrm{N}=6)$, pre-dialysis samples at baseline (median (IQR) hs-cTnT concentrations of 151.8(134.7-159.6) $\mathrm{ng} / \mathrm{L})$ and 2 months later (median (IQR) hs-cTnT concentrations of 131.8 (115.7-158.2) ng/L) were analyzed (SUPPLEMENTAL FIGURE 3). Also here, GFC profiles could only distinguish a reproducible peak at $45 \mathrm{~mL}$ for both time-points (FIGURE $3 \mathrm{C}$ and D).

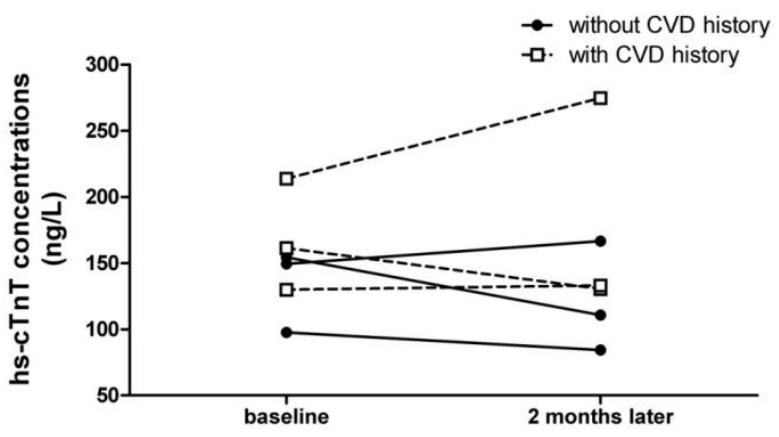

SUPPLEMENTAL FIGURE 3. Hs-cTnT concentrations measured in pre-hemodialysis serum samples of ESRD patients $(n=6)$ at baseline and 2 months later. 

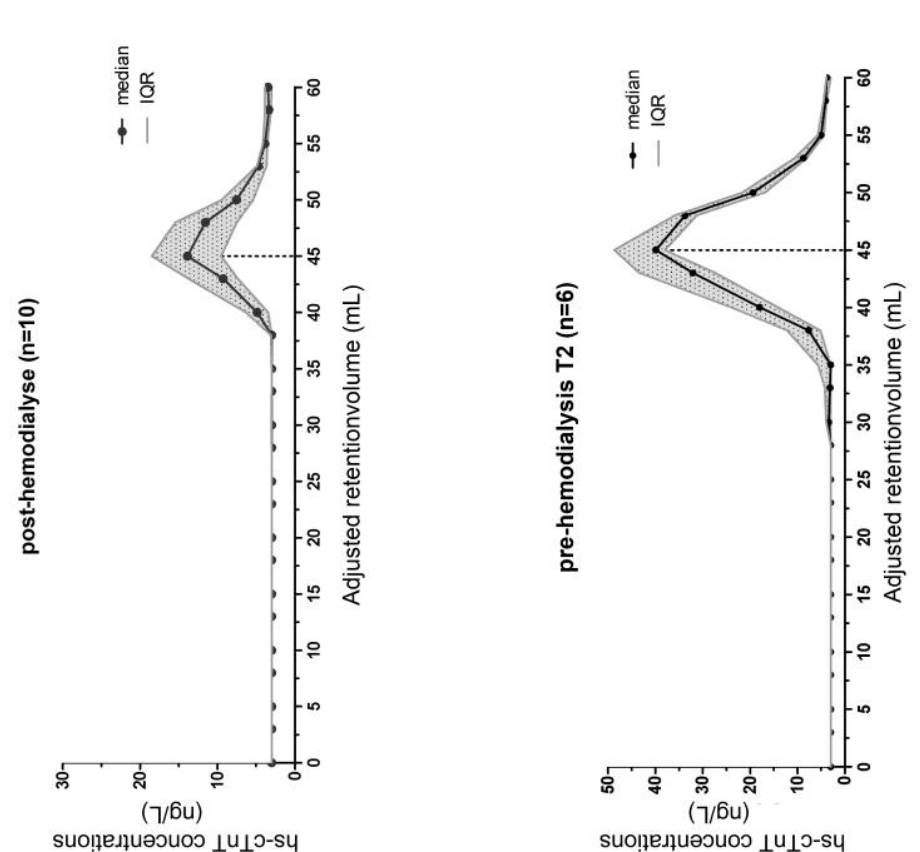

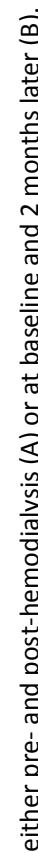
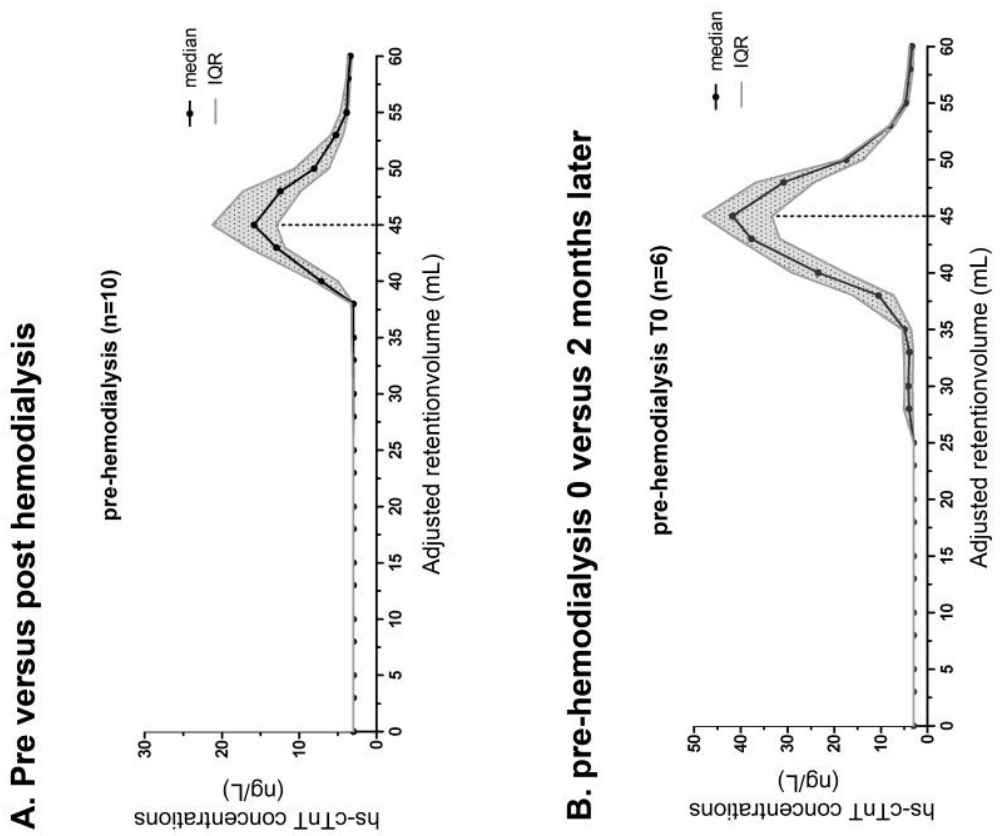

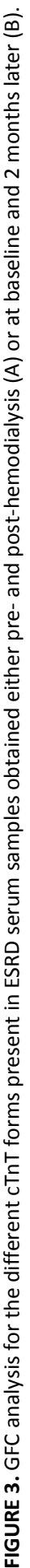




\section{Discussion}

In the present study, we clearly demonstrate that chronic cTnT elevations measured in ESRD patients' sera, only reflect degraded cTnT forms. Moreover, these cTnT-fragments were observed before and after hemodialysis, and were found to be stable over time.

By means of GFC analysis, we were able to distinguish ternary cTnT-I-C complexes from intact and degraded cTnT forms. Moreover, our technique could differentiate the $29 \mathrm{kDa}$ cTnT fragment from low-molecular weight $(<18$ $\mathrm{kDa}$ ) cTnT fragments. Only intact cTnT and the $29 \mathrm{kDa}$ cTnT form could not be completely separated, consequently requiring Western blotting analysis for further differentiation. Also, we confirmed earlier findings of a two peak cTnT profile following AMI, containing $29 \mathrm{kDa}$ and $<18 \mathrm{kDa}$ cTnT fragments [2].

In cases of ESRD, we repeatedly distinguished one cTnT-peak at $45 \mathrm{~mL}$, corresponding to the second peak in AMI patients, that was allocated to small $\mathrm{N}$-terminal truncated cTnT forms. These results therefore indicate that cTnT elevations merely reflect completely degraded cTnT fragments in cases of ESRD as previously suggested [11] and not intact cTnT as also assumed [12]. The truncation of the N-terminal end of cTnT has been described to occur in the intracellular environment of cardiomyocytes [14-16]. However, whether observed cTnT fragments presented in this study are formed intracellularly or extracellularly goes beyond the purpose of this study and merits further research. Furthermore, whether these low-molecular weight cTnT fragments still contain their C-terminus has yet to be explored.

In a second ESRD cohort, similar cTnT fragments were found in serum samples drawn 2 months apart [4]. Unlike the time-dependent cTnT degradation pattern following AMI [2, 17], cTnT fragments in ESRD patients' sera therefore seem more stable in time. Moreover, the composition of cTnT forms clearly differs between AMI and ESRD patients, mainly during the time frame of clinical diagnosis of AMI (<12 hours) [2]. Consequently, it would be interesting to assess whether future assays, focusing only on the $27.5 \mathrm{ml}$ peak, would increase the diagnostic performance of troponin assays in predicting acute coronary syndromes in patients with ESRD, which currently relies on serial hscTn measurements [18, 19]. 
Low-molecular weight cTnT forms were found predialysis and immediately after hemodialysis. A minor but non-significant decrease following hemodialysis in the cTnT GFC-peak was distinguished. It is therefore expected that these lowmolecular weight cTnT-fragments are in all probability cleared by the kidneys or during renal replacement therapy.

Monitoring the calibration and precision of the assay is necessary to ensure quality hs-cTnT measurements. As shown in the present study, the materials used for this purpose seem to contain only intact cTnT molecules. Therefore, these quality controls are clearly not representative for ESRD and AMI patient's serum. However, the magnitude of hs-cTnT measurements has still been described to be of prognostic value in ESRD [8, 9] as well as other populations [20-22]. It is therefore of utmost importance to investigate whether immunoreactivity changes for different cTnT forms and subsequently the detection of a mixture of cTnT forms could have implications for the clinical assay $[19,23]$.

In conclusion, this is to the first study to conclusively reveal that low-molecular weight cTnT fragments $(<18 \mathrm{kDa})$ are present in the serum of ESRD patients. Importantly, this is different from cTnT-forms are recognized by the clinical assay in the acute phase of AMI and in quality control materials. 


\section{References}

1. Thygesen, K., J.S. Alpert, A.S. Jaffe, et al., Third universal definition of myocardial infarction. Eur Heart J, 2012. 33(20): p. 2551-67.

2. Cardinaels, E.P., A.M. Mingels, T. van Rooij, et al., Time-dependent degradation pattern of cardiac troponin T following myocardial infarction. Clin Chem, 2013. 59(7): p. 1083-90.

3. Kelley, W.E., J.L. Januzzi, and R.H. Christenson, Increases of cardiac troponin in conditions other than acute coronary syndrome and heart failure. Clin Chem, 2009. 55(12): p. 2098112.

4. Jacobs, L.H., J. van de Kerkhof, A.M. Mingels, et al., Haemodialysis patients longitudinally assessed by highly sensitive cardiac troponin $\mathrm{T}$ and commercial cardiac troponin $\mathrm{T}$ and cardiac troponin I assays. Ann Clin Biochem, 2009. 46(Pt 4): p. 283-90.

5. Aakre, K.M., T. Roraas, P.H. Petersen, et al., Weekly and 90-minute biological variations in cardiac troponin T and cardiac troponin I in hemodialysis patients and healthy controls. Clin Chem, 2014. 60(6): p. 838-47.

6. Mishra, R.K., Y. Li, C. DeFilippi, et al., Association of cardiac troponin T with left ventricular structure and function in CKD. Am J Kidney Dis, 2013. 61(5): p. 701-9.

7. Breidthardt, T., J.O. Burton, A. Odudu, et al., Troponin T for the detection of dialysisinduced myocardial stunning in hemodialysis patients. Clin J Am Soc Nephrol, 2012. 7(8): p. 1285-92.

8. Apple, F.S., M.M. Murakami, L.A. Pearce, et al., Predictive value of cardiac troponin I and T for subsequent death in end-stage renal disease. Circulation, 2002. 106(23): p. 2941-5.

9. Hassan, H.C., K. Howlin, A. Jefferys, et al., High-sensitivity troponin as a predictor of cardiac events and mortality in the stable dialysis population. Clin Chem, 2014. 60(2): p. 389-98.

10. deFilippi, C., S.L. Seliger, W. Kelley, et al., Interpreting cardiac troponin results from highsensitivity assays in chronic kidney disease without acute coronary syndrome. Clin Chem, 2012. 58(9): p. 1342-51.

11. Diris, J.H., C.M. Hackeng, J.P. Kooman, et al., Impaired renal clearance explains elevated troponin T fragments in hemodialysis patients. Circulation, 2004. 109(1): p. 23-5.

12. Fahie-Wilson, M.N., D.J. Carmichael, M.P. Delaney, et al., Cardiac troponin T circulates in the free, intact form in patients with kidney failure. Clin Chem, 2006. 52(3): p. 414-20.

13. Bates, K.J., E.M. Hall, M.N. Fahie-Wilson, et al., Circulating immunoreactive cardiac troponin forms determined by gel filtration chromatography after acute myocardial infarction. Clin Chem, 2010. 56(6): p. 952-958.

14. Feng, H.Z., M. Chen, L.S. Weinstein, et al., Removal of the N-terminal extension of cardiac troponin I as a functional compensation for impaired myocardial beta-adrenergic signaling. J Biol Chem, 2008. 283(48): p. 33384-93.

15. Zhang, Z., B.J. Biesiadecki, and J.P. Jin, Selective deletion of the NH2-terminal variable region of cardiac troponin $\mathrm{T}$ in ischemia reperfusion by myofibril-associated mu-calpain cleavage. Biochemistry, 2006. 45(38): p. 11681-94.

16. Feng, H.Z., B.J. Biesiadecki, Z.B. Yu, et al., Restricted N-terminal truncation of cardiac troponin T: a novel mechanism for functional adaptation to energetic crisis. J Physiol, 2008. 586(14): p. 3537-50.

17. Michielsen, E.C., J.H. Diris, V.W. Kleijnen, et al., Investigation of release and degradation of cardiac troponin T in patients with acute myocardial infarction. Clin Biochem, 2007. 40(12): p. 851-5.

18. Biener, M., H.A. Katus, and E. Giannitsis, Challenges of serial troponin testing: a symphony in need for harmony. Int J Cardiol, 2013. 168(4): p. 4542.

19. Lippi, G. and G. Cervellin, Challenges of serial troponin testing: an unfinished symphony. Int J Cardiol, 2013. 168(4): p. 4397.

20. de Lemos, J.A., M.H. Drazner, T. Omland, et al., Association of troponin T detected with a highly sensitive assay and cardiac structure and mortality risk in the general population. JAMA, 2010. 304(22): p. 2503-12. 
21. Gravning, J., E.T. Askevold, S.H. Nymo, et al., Prognostic effect of high-sensitive troponin T assessment in elderly patients with chronic heart failure: results from the CORONA trial. Circ Heart Fail, 2014. 7(1): p. 96-103.

22. van Wijk, S., L. Jacobs, L.W. Eurlings, et al., Troponin T measurements by high-sensitivity vs conventional assays for risk stratification in acute dyspnea. Clin Chem, 2012. 58(1): p. 28492.

23. Katrukha, A.G., A.V. Bereznikova, V.L. Filatov, et al., Degradation of cardiac troponin I: implication for reliable immunodetection. Clin Chem, 1998. 44(12): p. 2433-40. 



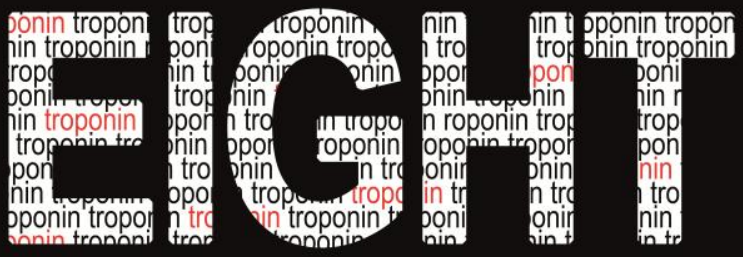

\section{GENERAL DISCUSSION}



There is a broad consensus regarding the use of cardiac troponins (cTn), either cTnT or cTnl, as the preferred biomarkers to identify acute myocardial infarction (AMI) [1]. Major analytical improvements to the cTn assays have resulted in more accurate and very sensitive information, giving rise to new opportunities. When measuring with high-sensitivity (hs-) assays, elevated hs-cTn concentrations were not solely detected in the context of AMI, but also in a whole range of other pathologies [2]. Due to this lack in disease-specificity, hscTn results can therefore be confusing for clinicians, especially in the low measuring range. In order for patients to fully profit from the benefits that these improved assays have to offer, it is of utmost importance to increase our understanding on how to interpret hs-cTn results.

\section{The trouble with defining $99^{\text {th }}$ percentile upper reference limits}

The current definition of an AMI is based on the rise and/or fall in cTn concentrations with at least one value above the $99^{\text {th }}$ percentile upper reference limit (URL) of normal [1]. In order to reliably assess the $99^{\text {th }}$ percentile with a uncertainty of $<5 \%$, the guidelines recommend a minimum sample size of 300 healthy subjects [3-5]. Furthermore, the reference population should be screened for the presence of cardiac diseases, ideally using cardiac imaging techniques. Also, due to a lack of harmonisation between cTn assays, $99^{\text {th }}$ percentile concentrations should be established for each assay [6].

Peer-reviewed literature on the real-life determinations of the $99^{\text {th }}$ percentile cutoff has been reviewed in chapter two. This chapter illustrates that variable selection criteria are used to define the reference population, and subsequently a wide range of $99^{\text {th }}$ percentile concentrations were found for each assay. Comparing these different reference populations, variations were identified in selection criteria, distribution of age and gender and the number of included individuals. Lack of attention regarding these issues can result in misleading decision cutoffs and can in this way influence clinical practice.

\section{The definition of a healthy reference population}

As described in chapter two, the concept of "healthy" varied between different publications, ranging from fully based on health questionnaires to extensive 
screens using laboratory and imaging tests. Applying more stringent selection criteria can result in a significant reduction in the $99^{\text {th }}$ percentile concentration [7-11]. However, applying rigorous screening tests such as echocardiography to a large number of presumably healthy subjects can be difficult and costly. Therefore, renal dysfunction combined with elevations in natriuretic peptides measurements [8], and left ventricular abnormalities assessed by echocardiography $[9,12]$ were reported to have the most pronounced effects on the $99^{\text {th }}$ percentile URL. Importantly, in chapter three it has been emphasized that even decreases within a normal kidney function can affect hs-cTn concentrations. As to date, no clear definition on what constitutes a healthy reference population for the determination of hs-cTn cutoffs has been described [13].

\section{The influence of age, gender and race}

Numerous publications, as illustrated in chapter two, include variable age and gender distributions within their reference cohort. Previous studies have demonstrated that males have significantly higher hs-cTn concentrations than females [8-12, 14], which is also displayed in chapter three. The rationale behind this could be that males have more heart tissue than females [15]. In chapter four, we showed that elevated hs-cTn concentrations are commonly found in the elderly even when contributing comorbidities were excluded. These findings extend recent literature on the significant influence of age on hs-cTn concentrations $[9,16,17]$ and further underline the need for gender and agespecific cutoffs in clinical practice as recently reviewed [18]. Although, large clinical trials investigating the diagnostic benefits of using these specific cutoffs are still lacking [19].

\section{How many healthy individuals should be included?}

When establishing the $99^{\text {th }}$ percentile concentrations, it appeared that many studies include less than 300 subjects (chapter two). Noticeably, in these small reference cohorts, subjects were more likely to be highly screened for the absence of cardiovascular diseases, whereas large reference populations were less rigorously screened (by means of health questionnaires). However, to ensure an accurate cutoff, enough patients should be included as the $99^{\text {th }}$ 
percentile concentration is extremely sensitive to outliers [5]. Importantly, when identifying gender and age specific cutoffs, at least 300 subjects should also be included in each subcategory [15].

\section{Association of renal clearance and high-sensitivity cardiac troponins}

One of the most common pathologies, besides AMI, for elevated CTn concentrations includes chronic kidney disease (CKD) and end-stage renal disease (ESRD) [2, 20]. In contrast to AMI, ESRD patients exhibit elevated hscTn levels that are more stable over time [21, 22]. The explanation of these chronic cTn elevations is still incompletely understood, and subclinical cardiac diseases are the most obvious explanation. However, a recent study including patients with renal dysfunction but without acute coronary syndromes, hs-cTn concentrations were still elevated and highly associated to renal clearance [23]. This renal influence also becomes evident in the study described in chapter five, where the effect of hemodialysis and the more promising hemodiafiltration treatments on hs-cTn concentrations are investigated. In this chapter a significant reduction in hs-cTnT as well as hs-cTnl concentrations following hemodiafiltration is described, as measured in serum samples drawn before and after hemodiafiltration. When patients received conventional hemodialysis, however no significant reduction in both hs-cTn concentrations could be established. This difference can be attributed to the presence of convection in hemodiafilatrion and not hemodialysis, which is known to induce more uremic toxin removal from the patients' circulation [24]. Together these data validate the hypothesis that cTn molecules are cleared by the kidneys. In principle, both cTn molecules are small enough to be filtered by the kidneys (37 and $24 \mathrm{kDa}$, respectively). For final elucidation, hs-cTn concentrations should however be measured in urine or dialysate samples, as reported for NTproBNP [25, 26]. Up till now, this remains difficult due to matrix effects and dilution of already very low serum cTn concentrations by urine or dialysate. Importantly, in mild CKD to ESRD patients, hs-cTn elevations should not be discarded as irrelevant, since they remain highly associated with increased risk of death and cardiovascular disease, even when adjusted for renal dysfunction [6, 27, 28]. 
As to date, the magnitude of the impact of decreased renal function on hs-cTn concentrations is unknown. In chapter three we increased our understanding on the cardiac and renal contribution to higher hs-cTnT and hs-cTnl concentrations in patients with chest pain, without any signs of renal disease. Herein, a significant inverse association between eGFR and hs-cTn was shown, which has also been established in other study populations [27, 29, 30]. Moreover, in chapter three, we revealed that the association with renal function was independent and stronger than with any measured CT parameters, such as CAD severity and coronary calcium score. Similar analyses were performed in an elderly population of nursing home residents (chapter four), with prevalent heart failure $(\sim 40 \%)$ and renal dysfunction ( $54 \%)$. Also here, hs-cTn concentrations were highly associated with renal dysfunction even beyond the presence of heart failure. Together, these studies demonstrate that hs-cTn concentrations, especially hs-cTnT, reflect both renal and cardiac disease and subsequently both pathologies can greatly affect the $99^{\text {th }}$ percentile URL. Furthermore, these findings could offer an explanation why diagnostic cutoffs established in cardiac and renal healthy subjects have a reduced diagnostic performance in patients with renal dysfunction [31]. Therefore, further research should focus on the influence of renal dysfunction on cTn cutoff values and investigate whether adjusting cTn cutoffs for renal function is of clinical value or baseline hs-cTn results should be accompanied with renal function assessment.

\section{Proteolysis of cardiac troponin T}

Injury to the myocardium can result in the activation and release of proteolytic enzymes. For cTnl it is generally acknowledged that proteolysis following myocardial infarction results in the presence of different cTnl fragments in human serum [32-34]. This diversity in molecular forms of cTnl had serious implications for the harmonisation between different cTnl assays [34, 35]. Since all cTnl assays employed different antibodies that recognize different cTnl forms, up to 10-fold variation between cTnl-results assays could be observed. For this reason it is necessary that antibodies should recognize epitopes in the most stable parts of cTnl [34,35], resulting in considerably less variations in cTnl nowadays. 
In contrast to cTnl, degradation of cTnT upon myocardial infarction remained controversial. By means of Western blotting, but employing other antibodies as the clinical cTnT assay, the presence of degraded cTnT forms has been shown in serum of AMI patients [36,37]. Also, in serum of ESRD patients, only cTnT fragments were found [23]. However, these results were disputed in studies using gel filtration chromatography (GFC), who found cTnT in serum of AMI [38] and ESRD patients [39] to be mainly in the intact or complexed form with cTnl or TnC. Therefore, a combination of GFC and Western blotting, using the same antibodies as the clinical assay, was utilized in chapter six and chapter seven to finally elucidate the cTnT forms that circulate in AMI and ESRD patients' sera, respectively.

\section{Time-dependent degradation of cTnT}

In chapter six we demonstrate that a heterogeneous mixture of cTn fragments was found in the blood of AMI patients and these fragments are identified with the clinical cTnT assay antibodies. It was shown that not intact cTnT, but the Nterminal truncated form of cTnT, corresponding to a molecular weight of $\sim 29$ $\mathrm{kDa}$, was most abundantly present during the critical hours of diagnostic testing (<8 hours). Thereafter, smaller fragments ranging in size from 14-18 kDa appeared to circulate in AMI patients' sera, validating the time-dependent degradation pattern that was previously described [36].

In chapter seven, we found in ESRD patients with chronic cTnT elevations that exclusively low-molecular weight fragments $(<18 \mathrm{kDa})$ were present in the circulation. The same fragments were found for all ESRD patients (total of 17 patients) in samples drawn at different timepoints (at 2 months of follow up and pre- versus post-dialysis). It should be noted that these ESRD patients were treated with conventional hemodialysis, which was shown in chapter five to not significantly influence hs-cTn concentrations pre- versus post dialysis. In contrast, a significant intradialytic decrease in hs-cTnT concentrations was shown following hemodiafiltration (chapter five). It would therefore be interesting to also examine cTnT-fragments that are present pre- versus posthemodiafiltration. 


\section{Implications for the clinical assay results}

Since, Roche Diagnostics is the only manufacturer of clinical cTnT assays, cTnT degradation does not affect harmonization between different assays. To ensure quality of the cTnT measurement in blood samples, the manufacturer recommends the use of recombinant human cTnT spiked in a human serum matrix for monitoring the calibration and precision of the assay. However, as shown in this thesis, recombinant intact cTnT is not representative for AMI and ESRD patients' serum, which was clearly shown to mainly contain cTnT fragments.

It is currently unknown whether immunoreactivity of the assay is equal for different cTnT forms. It seems very likely that the assay response to the various troponin forms might differ and, consequently, will affect the performance of serial measurements [40]. Whether the time-dependent degradation pattern influences the diagnostic performance of cTnT, still needs further clinical evaluation [41].

Nevertheless, in this thesis it was clearly shown that the most prevalent molecular forms differed between AMI ( $29 \mathrm{kDa})$ and ESRD (<18 kDa) patients. These findings have led to the hypothesis that the cTnT assay could be improved by recognizing specific molecular cTnT forms, and thereby make a better differentiation between acute and chronic cTnT elevations.

\section{Intracellular or extracellular degradation of cTnT}

It still remains to be elucidated whether the observed degradation of cTnT occurs intracellular or extracellular of ischemic cardiomyocytes. Several in vitro studies have demonstrated that the degradation of CTnT occurred upon ischemia, due to the activity of either calpain-I [42-44] and caspase-3 [45] in the intracellular environment of cultured cardiomyocytes. This intracellular proteolytic activity is shown to result in a N-terminal truncated form of cTnT, that does not impair the function of cTnT but reflects an intracellular adaption to stress conditions [43]. In contrast, in vitro studies on metabolic deprived cardiomyocytes of primary cell cultures [46] and a contracting cell-line [47] have demonstrated that after cell death mostly intact cTnT and only minor fragments are detected in the medium of the cell culture. Current literature therefore 
suggests not only a role for intracellular but also extracellular proteolytic activity. This all leads to the intriguing question of which modification patterns of cTn are present when myocardial necrosis is thought to be absent, for instance after physical activity. Moreover, understanding the mechanisms that result in these post-translational modifications of cTn can give further insights into the release of cTn from cardiomyocytes.

\section{Differences between cTnT and cTnl}

Throughout this thesis, hs-cTnT and hs-cTnl were measured simultaneously in different patient cohorts (TABLE 1). Interestingly, in each cohort we observed that median hs-cTnT concentrations were noticeably higher than hs-cTnl concentrations. This was consistent with data published in peer-reviewed articles who also investigated hs-cTnT and hs-cTnl concentrations in ACS and non-ACS patients (TABLE 2). On the other hand, in AMI patients hs-cTnl concentrations were found to be markedly higher than hs-cTnT and were only moderately associated with each other (Pearson R 0.60) (TABLE 2) [48-51].

Despite the fact that differences between hs-cTnT and hs-cTnl have been described, no detailed analysis regarding the differences in chronic versus acute diseases is present to date. Moreover, according to the current guidelines, cTnT and cTnl results are considered interchangeably from each other [4]. 


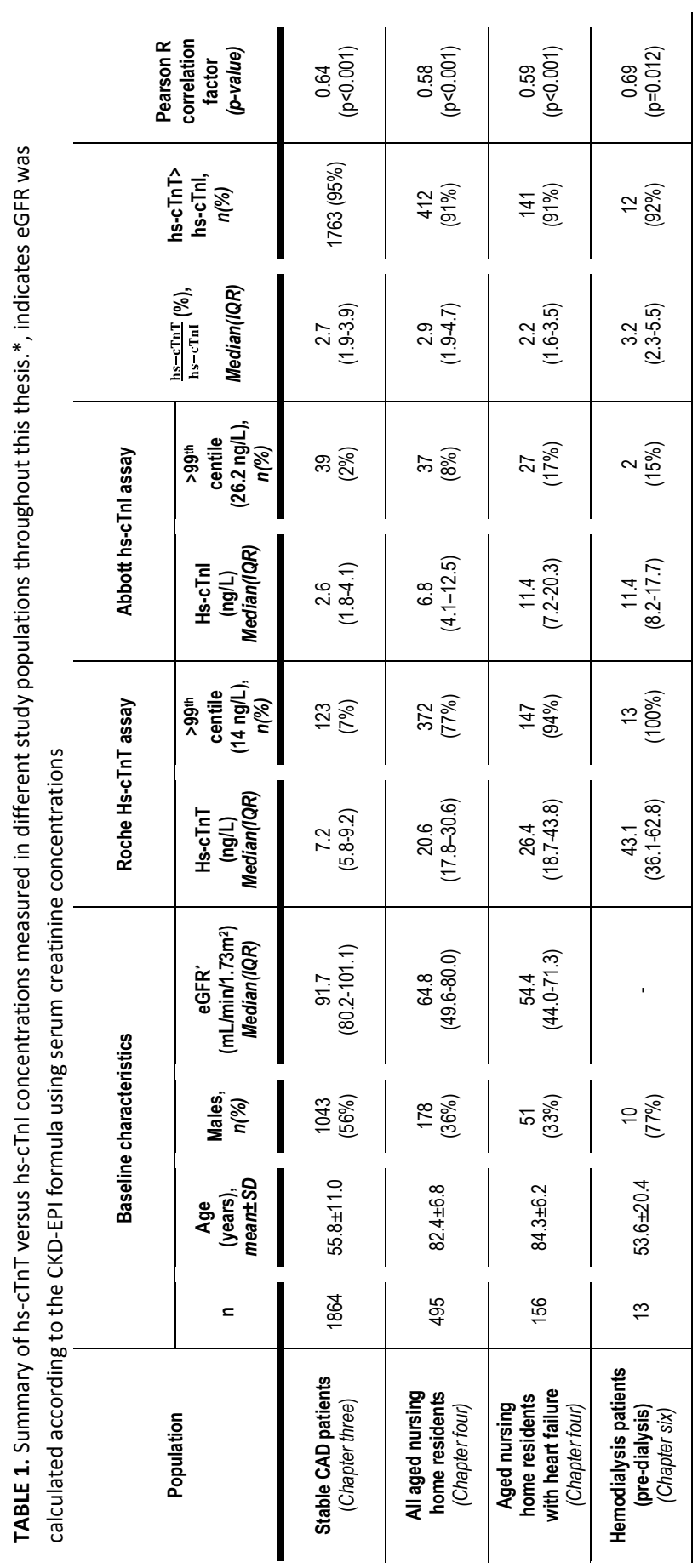




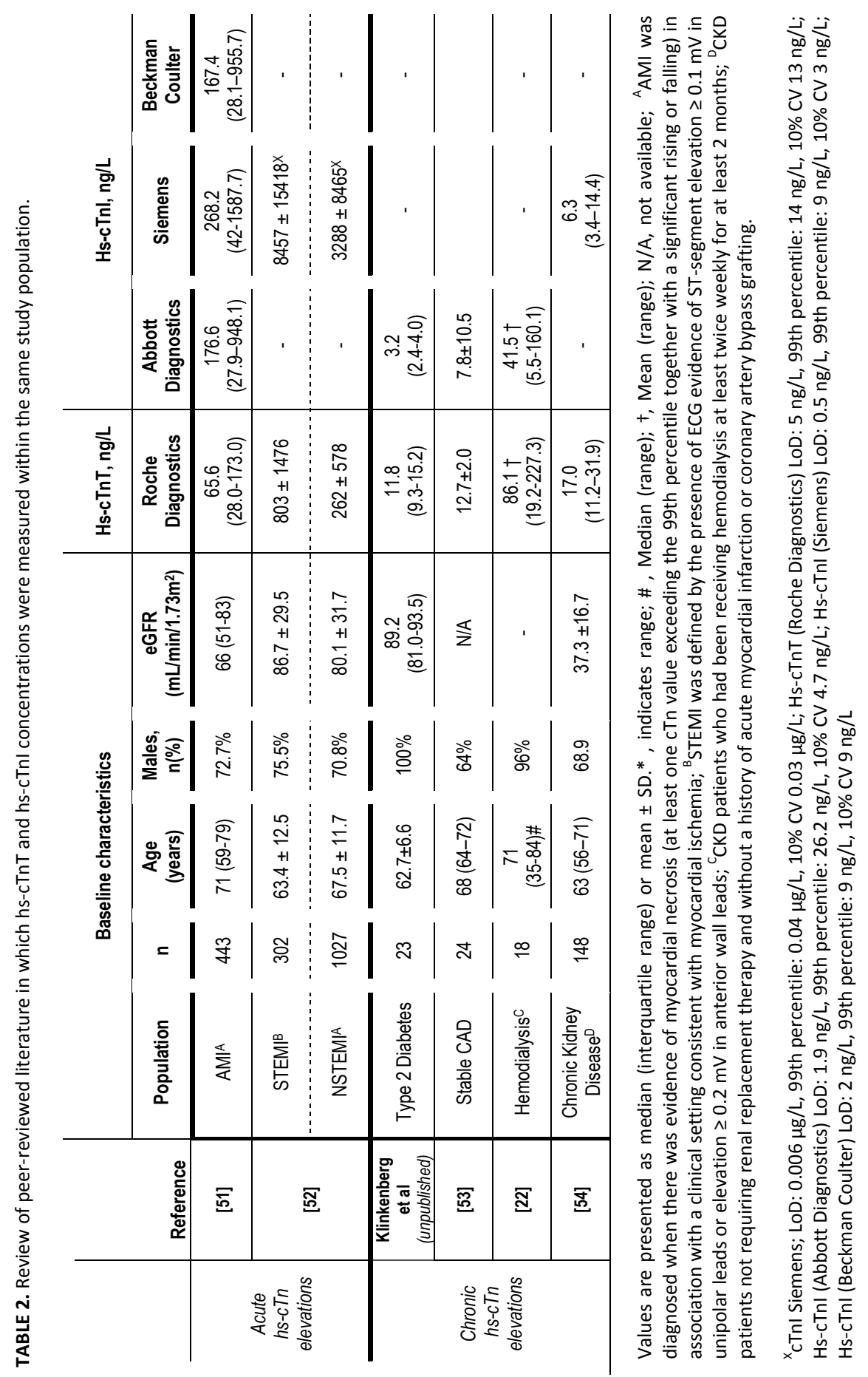




\section{Release kinetics of cTnT versus cTnI molecules}

The release kinetics of $\mathrm{cTnl}$ have been described to deviate from cTnT in both earlier release [52] and decline [53] following myocardial damage. Namely, cTnT exhibits a second peak that has been allocated to a slower dissociation of cTnT molecules due to its structural bound to myofibrils, which is absent for cTnl $[53,54]$. Whether also more cTnl molecules are therefore released upon myocardial damage is currently unknown.

In chapter three and chapter four we observed that hs-cTnT was more strongly associated to renal dysfunction in comparison to hs-cTnl. In line with this finding, excluding patients with renal dysfunction had the most effects on hscTnT in comparison to hs-cTnl (chapter four). More robust associations of eGFR with hs-cTnT in comparison to hs-cTnl have also been reported by others [8, 55] and generally there are more elevations reported for cTnT than cTnl in ESRD patients [21, 56]. Together, these findings evidence that hs-cTnT results are more susceptible to changes in renal function than hs-cTnl results. To our opinion, the second peak in the release curve of cTnT might therefore be attributed to the accumulation of cTnT molecules following a slower clearance from the system in comparison to cTnl.

Additionally, in chapter five, it was found that the influence of hemodialysis differed between hs-cTnT and hs-cTnl concentrations. Although, it remains difficult to separate the effects of release and clearance on higher hs-cTn concentrations, hemodiafiltration was shown to have more effect on hs-cTnT in comparison to hs-cTnl. Structural differences between cTnT and cTnl can contribute to these dissimilarities as the molecular size of cTnl (24 kDa) is smaller than cTnT (37 kDa). Additionally, it is known that both proteins are degraded in the patients serum, resulting in a heterogeneous mixture of cTnT (<29 kDa, chapter six and chapter seven) and cTnl (<20 kDa; [32, 34]) molecules. Due to these structural differences, release from damaged myocardium [53] and transfer of cTnT and cTnl across the viable cell membrane may not be equal. 


\section{Prognostic value of hs-cTnT versus hs-cTnl concentrations}

Several large clinical trials have demonstrated that low hs-cTnT and hs-cTnl concentrations, even below the diagnostic cutoff, are associated with a higher risk on developing cardiovascular events and all-cause mortality, and even on top of traditional risk factors $[30,55,57]$. In chapter four, hs-cTnT was found to be a superior predictor for all-cause mortality in comparison to hs-cTnl. These findings have also been observed in the APACE trial, enrolling patients at the emergency department with symptoms suggestive for AMI [58]. In these patients, risk on mortality was more accurately predicted by hs-cTnT than by hscTnl. Similarly, in other populations such as ESRD [6], heart failure [59] and ACS [60] patients, hs-cTnT was found to be a better predictor for all-cause mortality than cTnl assays. In line with these results, Omland and colleagues found that hs-cTnT and hs-cTnl can hold independent prognostic information to each other [55], questioning whether cTnT and cTnl are truly interchangeable risk predictors. The reasoning behind these observed differences is still speculative and could involve the stronger association of hs-cTnT with renal disease. Remarkably, for the prediction of cardiovascular disease these differences were less apparent than for all-cause mortality, as demonstrated in chapter three [58]. More studies involving both hs-cTnT as hs-cTnl assays should therefore be performed for final elucidation of these differences.

\section{Cardiospecificity of cTnT versus cTnl proteins}

Since the discovery of cTn proteins as cardiac biomarkers, it has been shown that the cardiac isoform of cTn is specifically expressed in cardiac muscle [61, 62]. However, the cardiospecificity of cTnT, but not cTnl, has been questioned especially in patients with neuromuscular diseases [63]. In these patients, cTnT results were elevated whereas normal (<URL) cTnl concentrations were detected. In these studies, the rationale for this phenomenon was the reexpression of the cardiac cTnT isoform in the skeletal muscles, resulting in false-positive cTnT elevations $[64,65]$. In this thesis, we demonstrate that hscTnT results are in general higher than hs-cTnl results. Moreover, elevated cTn concentrations are defined by the $99^{\text {th }}$ percentile upper reference limit, which is 
highly dependent on the reference population and mostly reported to be lower for cTnT as for cTnl [15].

Using western blotting it was shown that the Roche capture and detector antibodies are each capable of detecting cTnT in skeletal biopsies of patients with neuromuscular disorders [64]. However, to ensure minimal aspecific binding of the assay, the clinical cTnT assay encompasses the combination of the two antibodies. Due to the a kind collaboration with Roche Diagnostics, we did not found any aspecific binding towards skeletal forms of troponin $\mathrm{T}$ using Western blotting incorporating both clinical (capture and detection) antibodies. Also, hs-cTnT and hs-cTnl were shown to correlate equally as well to cardiac diseases such as AMI and heart failure. Therefore, no hard evidence is currently given to question the cardiospecificity of the cTnT assay.

\section{Conclusion}

It is apparent from data in the present thesis that the $99^{\text {th }}$ percentile is not a perfect diagnostic cutoff and has its limitations concerning the influences of age, gender and renal function of the cardio-healthy reference population. Noteworthy is that hs-cTn concentrations are no stand-alone tests and should always be interpreted in the clinical context together with clinical symptoms and additional diagnostic testing [1].

Although the interpretation of hs-cTn results is complex, in this thesis we observed that both hs-cTnT and hs-cTnl concentrations are still highly associated with cardiac diseases and even very low levels were valuable risk stratifying tools. Our results however also show that next to cardiovascular disease, hs-cTn concentrations, especially hs-cTnT, are highly associated with renal function as well.

Furthermore, we clearly demonstrate that upon its release from the damaged myocardium, cTnT is degraded in a time-dependent manner, ranging from intact to low-molecular weight fragments, which are all recognized by the antibodies of the clinical assay (FIGURE 1). The smallest degradation products also accumulate in the circulation of ESRD patients, and in this form, cTnT molecules are presumably cleared from the circulation (FIGURE 1). 


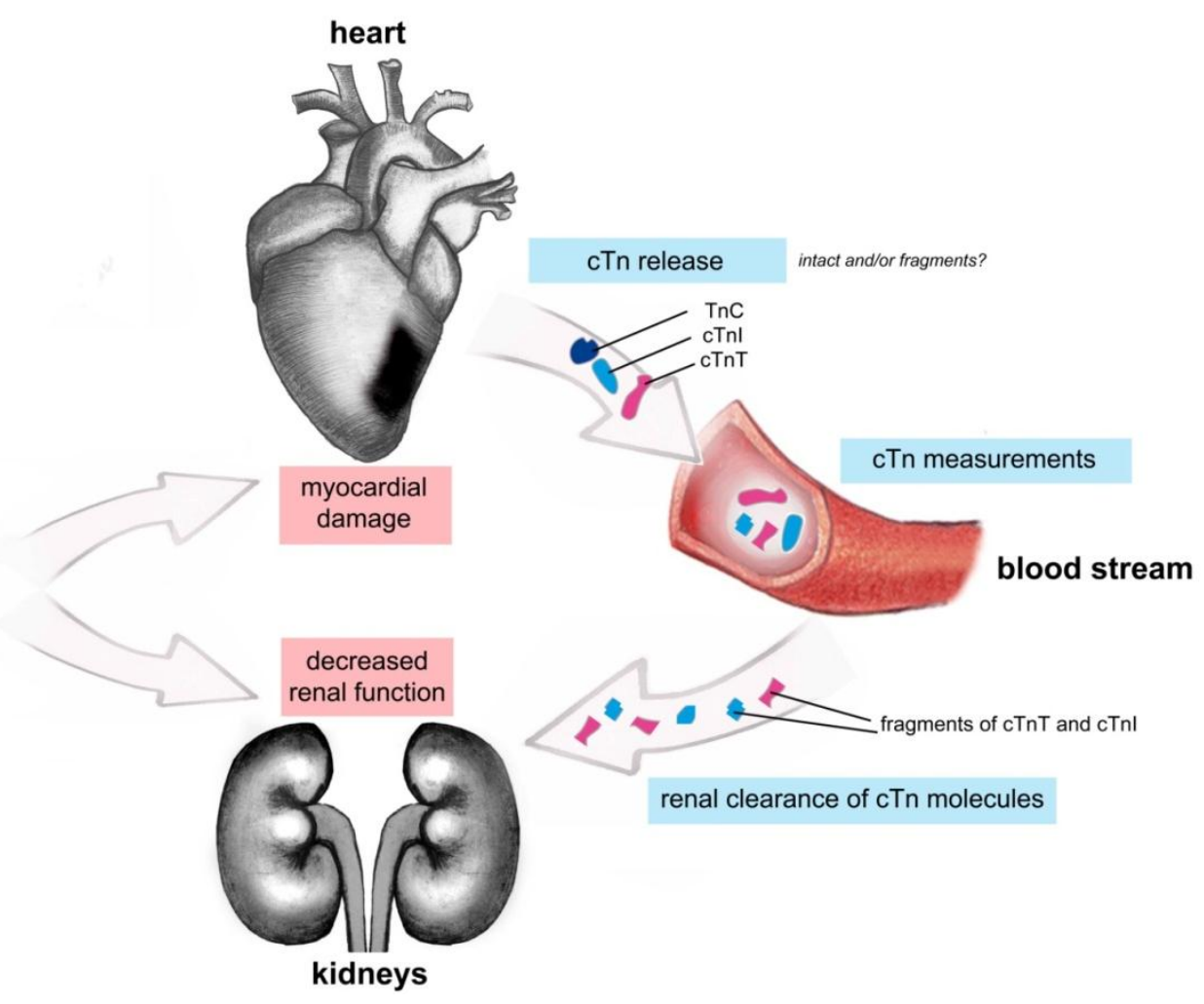

FIGURE 1. Following myocardial damage, cTn molecules are released into the blood stream. In the patients' circulation, degraded cTnT (chapter seven and eight) and cTnl [33] forms have been detected. We hypothesize that these low-molecular weight cTnT and cTnl fragments will eventually be cleared from the circulation via the kidneys. However, these influences are complicated by the fact that release and clearance of cTn are simultaneous processes and cardiac and renal disease can aggravate each other.

Finally, we observed remarkable differences between hs-cTnT and hs-cTnl concentrations in all our clinical populations, seriously questioning whether these results are truly interchangeable from each other. Future work should illustrate in more detail if a combination of both cTnT and cTnl testing can have added diagnostic value in more complex cases. 


\section{References}

1. Thygesen, K., J.S. Alpert, A.S. Jaffe, et al., Third universal definition of myocardial infarction. Eur Heart J, 2012. 33(20): p. 2551-67.

2. Kelley, W.E., J.L. Januzzi, and R.H. Christenson, Increases of cardiac troponin in conditions other than acute coronary syndrome and heart failure. Clin Chem, 2009. 55(12): p. 2098112.

3. Apple, F.S., R.L. Jesse, L.K. Newby, et al., National Academy of Clinical Biochemistry and IFCC Committee for Standardization of Markers of Cardiac Damage Laboratory Medicine Practice Guidelines: analytical issues for biochemical markers of acute coronary syndromes. Clin Chem, 2007. 53(4): p. 547-51.

4. Thygesen, K., J. Mair, H. Katus, et al., Recommendations for the use of cardiac troponin measurement in acute cardiac care. Eur Heart J, 2010. 31(18): p. 2197-204.

5. Hickman, P.E., T. Badrick, S.R. Wilson, et al., Reporting of cardiac troponin - problems with the 99th population percentile. Clin Chim Acta, 2007. 381(2): p. 182-3.

6. Apple, F.S., M.M. Murakami, L.A. Pearce, et al., Predictive value of cardiac troponin I and T for subsequent death in end-stage renal disease. Circulation, 2002. 106(23): p. 2941-5.

7. Franzini, M., V. Lorenzoni, S. Masotti, et al., The calculation of the cardiac troponin T 99th percentile of the reference population is affected by age, gender, and population selection: A multicenter study in Italy. Clin Chim Acta, 2015. 438: p. 376-81.

8. Collinson, P.O., Y.M. Heung, D. Gaze, et al., Influence of population selection on the 99th percentile reference value for cardiac troponin assays. Clin Chem, 2012. 58(1): p. 219-25.

9. Eggers, K.M., L. Lind, P. Venge, et al., Factors influencing the 99th percentile of cardiac troponin I evaluated in community-dwelling individuals at 70 and 75 years of age. Clin Chem, 2013. 59(7): p. 1068-73.

10. Koerbin, G., W.P. Abhayaratna, J.M. Potter, et al., Effect of population selection on 99th percentile values for a high sensitivity cardiac troponin I and T assays. Clin Biochem, 2013. 46(16-17): p. 1636-43.

11. Zeller, T., F. Ojeda, F.J. Brunner, et al., High-sensitivity cardiac troponin I in the general population - defining reference populations for the determination of the 99th percentile in the Gutenberg Health Study. Clin Chem Lab Med, 2014.

12. McKie, P.M., D.M. Heublein, C.G. Scott, et al., Defining high-sensitivity cardiac troponin concentrations in the community. Clin Chem, 2013. 59(7): p. 1099-107.

13. Sandoval, Y. and F.S. Apple, The global need to define normality: the 99th percentile value of cardiac troponin. Clin Chem, 2014. 60(3): p. 455-62.

14. Hammarsten, O., M.L. Fu, R. Sigurjonsdottir, et al., Troponin T percentiles from a random population sample, emergency room patients and patients with myocardial infarction. Clin Chem, 2012. 58(3): p. 628-37.

15. Apple, F.S., P.O. Collinson, and I.T.F.o.C.A.o.C. Biomarkers, Analytical characteristics of highsensitivity cardiac troponin assays. Clin Chem, 2012. 58(1): p. 54-61.

16. Gore, M.O., S.L. Seliger, C.R. Defilippi, et al., Age- and sex-dependent upper reference limits for the high-sensitivity cardiac troponin T assay. J Am Coll Cardiol, 2014. 63(14): p. 1441-8.

17. Kuster, N., K. Monnier, G. Baptista, et al., Estimation of age- and comorbidities-adjusted percentiles of high-sensitivity cardiac troponin T levels in the elderly. Clin Chem Lab Med, 2014.

18. Jaffe, A.S. and F.S. Apple, High-sensitivity cardiac troponin assays: isn't it time for equality? Clin Chem, 2014. 60(1): p. 7-9.

19. Motiwala, S.R., A. Sarma, J.L. Januzzi, et al., Biomarkers in ACS and heart failure: should men and women be interpreted differently? Clin Chem, 2014. 60(1): p. 35-43.

20. Group, N.W., A.H. Wu, A.S. Jaffe, et al., National Academy of Clinical Biochemistry laboratory medicine practice guidelines: use of cardiac troponin and B-type natriuretic peptide or $\mathrm{N}$-terminal proB-type natriuretic peptide for etiologies other than acute coronary syndromes and heart failure. Clin Chem, 2007. 53(12): p. 2086-96. 
21. Jacobs, L.H., J. van de Kerkhof, A.M. Mingels, et al., Haemodialysis patients longitudinally assessed by highly sensitive cardiac troponin $\mathrm{T}$ and commercial cardiac troponin $\mathrm{T}$ and cardiac troponin I assays. Ann Clin Biochem, 2009. 46(Pt 4): p. 283-90.

22. Aakre, K.M., T. Roraas, P.H. Petersen, et al., Weekly and 90-minute biological variations in cardiac troponin T and cardiac troponin I in hemodialysis patients and healthy controls. Clin Chem, 2014. 60(6): p. 838-47.

23. Diris, J.H., C.M. Hackeng, J.P. Kooman, et al., Impaired renal clearance explains elevated troponin T fragments in hemodialysis patients. Circulation, 2004. 109(1): p. 23-5.

24. Cornelis, T., F.M. van der Sande, S. Eloot, et al., Acute hemodynamic response and uremic toxin removal in conventional and extended hemodialysis and hemodiafiltration: a randomized crossover study. Am J Kidney Dis, 2014. 64(2): p. 247-56.

25. Jungbauer, C.G., S. Buchner, C. Birner, et al., N-terminal pro-brain natriuretic peptide from fresh urine for the biochemical detection of heart failure and left ventricular dysfunction. Eur J Heart Fail, 2010. 12(4): p. 331-7.

26. Palmer, S.C., Z.H. Endre, A.M. Richards, et al., Characterization of NT-proBNP in human urine. Clin Chem, 2009. 55(6): p. 1126-34.

27. Scheven, L., P.E. de Jong, H.L. Hillege, et al., High-sensitive troponin T and N-terminal pro-B type natriuretic peptide are associated with cardiovascular events despite the crosssectional association with albuminuria and glomerular filtration rate. Eur Heart J, 2012. 33(18): p. 2272-81.

28. Hassan, H.C., K. Howlin, A. Jefferys, et al., High-sensitivity troponin as a predictor of cardiac events and mortality in the stable dialysis population. Clin Chem, 2014. 60(2): p. 389-98.

29. Tsutamoto, T., C. Kawahara, M. Yamaji, et al., Relationship between renal function and serum cardiac troponin T in patients with chronic heart failure. Eur J Heart Fail, 2009. 11(7): p. 653-8.

30. de Lemos, J.A., M.H. Drazner, T. Omland, et al., Association of troponin T detected with a highly sensitive assay and cardiac structure and mortality risk in the general population. JAMA, 2010. 304(22): p. 2503-12.

31. Chenevier-Gobeaux, C., C. Meune, Y. Freund, et al., Influence of age and renal function on high-sensitivity cardiac troponin T diagnostic accuracy for the diagnosis of acute myocardial infarction. Am J Cardiol, 2013. 111(12): p. 1701-7.

32. Morjana, N.A., Degradation of human cardiac troponin I after myocardial infarction. Biotechnol Appl Biochem, 1998. 28 ( Pt 2): p. 105-11.

33. Madsen, L.H., G. Christensen, T. Lund, et al., Time course of degradation of cardiac troponin I in patients with acute ST-elevation myocardial infarction: the ASSENT-2 troponin substudy. Circ Res, 2006. 99(10): p. 1141-7.

34. Katrukha, A.G., A.V. Bereznikova, V.L. Filatov, et al., Degradation of cardiac troponin I: implication for reliable immunodetection. Clin Chem, 1998. 44(12): p. 2433-40.

35. Shi, Q., M. Ling, X. Zhang, et al., Degradation of cardiac troponin I in serum complicates comparisons of cardiac troponin I assays. Clin Chem, 1999. 45(7): p. 1018-25.

36. Michielsen, E.C., J.H. Diris, V.W. Kleijnen, et al., Investigation of release and degradation of cardiac troponin $\mathrm{T}$ in patients with acute myocardial infarction. Clin Biochem, 2007. 40(12): p. 851-5.

37. Labugger, R., L. Organ, C. Collier, et al., Extensive troponin I and T modification detected in serum from patients with acute myocardial infarction. Circulation, 2000. 102(11): p. 1221-6.

38. Bates, K.J., E.M. Hall, M.N. Fahie-Wilson, et al., Circulating immunoreactive cardiac troponin forms determined by gel filtration chromatography after acute myocardial infarction. Clin Chem, 2010. 56(6): p. 952-8.

39. Fahie-Wilson, M.N., D.J. Carmichael, M.P. Delaney, et al., Cardiac troponin T circulates in the free, intact form in patients with kidney failure. Clin Chem, 2006. 52(3): p. 414-20.

40. Lippi, G. and G. Cervellin, Challenges of serial troponin testing: an unfinished symphony. Int J Cardiol, 2013. 168(4): p. 4397.

41. Biener, M., H.A. Katus, and E. Giannitsis, Challenges of serial troponin testing: a symphony in need for harmony. Int J Cardiol, 2013. 168(4): p. 4542. 
42. Ke, L., X.Y. Qi, A.J. Dijkhuis, et al., Calpain mediates cardiac troponin degradation and contractile dysfunction in atrial fibrillation. J Mol Cell Cardiol, 2008. 45(5): p. 685-93.

43. Feng, H.Z., B.J. Biesiadecki, Z.B. Yu, et al., Restricted N-terminal truncation of cardiac troponin T: a novel mechanism for functional adaptation to energetic crisis. J Physiol, 2008. 586(14): p. 3537-50.

44. Zhang, Z., B.J. Biesiadecki, and J.P. Jin, Selective deletion of the NH2-terminal variable region of cardiac troponin $\mathrm{T}$ in ischemia reperfusion by myofibril-associated mu-calpain cleavage. Biochemistry, 2006. 45(38): p. 11681-94.

45. Communal, C., M. Sumandea, P. de Tombe, et al., Functional consequences of caspase activation in cardiac myocytes. Proc Natl Acad Sci U S A, 2002. 99(9): p. 6252-6.

46. Hessel, M.H., E.C. Michielsen, D.E. Atsma, et al., Release kinetics of intact and degraded troponin I and T after irreversible cell damage. Exp Mol Pathol, 2008. 85(2): p. 90-5.

47. Streng, A.S., L.H. Jacobs, R.W. Schwenk, et al., Cardiac troponin in ischemic cardiomyocytes: intracellular decrease before onset of cell death. Exp Mol Pathol, 2014. 96(3): p. 339-45.

48. Reiter, M., R. Twerenbold, T. Reichlin, et al., Early diagnosis of acute myocardial infarction in patients with pre-existing coronary artery disease using more sensitive cardiac troponin assays. Eur Heart J, 2012. 33(8): p. 988-97.

49. Reichlin, T., W. Hochholzer, S. Bassetti, et al., Early diagnosis of myocardial infarction with sensitive cardiac troponin assays. N Engl J Med, 2009. 361(9): p. 858-67.

50. Reiter, M., R. Twerenbold, T. Reichlin, et al., Early diagnosis of acute myocardial infarction in the elderly using more sensitive cardiac troponin assays. Eur Heart J, 2011. 32(11): p. 137989.

51. Rubini Gimenez, M., R. Hoeller, T. Reichlin, et al., Rapid rule out of acute myocardial infarction using undetectable levels of high-sensitivity cardiac troponin. Int J Cardiol, 2013. 168(4): p. 3896-901.

52. Rubini Gimenez, M., R. Twerenbold, T. Reichlin, et al., Direct comparison of high-sensitivitycardiac troponin I vs. T for the early diagnosis of acute myocardial infarction. Eur Heart J, 2014. 35(34): p. 2303-11.

53. Solecki, K., A.M. Dupuy, N. Kuster, et al., Kinetics of high-sensitivity cardiac troponin T or troponin I compared to creatine kinase in patients with revascularized acute myocardial infarction. Clin Chem Lab Med, 2014.

54. Katus, H.A., A. Remppis, T. Scheffold, et al., Intracellular compartmentation of cardiac troponin $\mathrm{T}$ and its release kinetics in patients with reperfused and nonreperfused myocardial infarction. Am J Cardiol, 1991. 67(16): p. 1360-7.

55. Omland, T., M.A. Pfeffer, S.D. Solomon, et al., Prognostic value of cardiac troponin I measured with a highly sensitive assay in patients with stable coronary artery disease. J Am Coll Cardiol, 2013. 61(12): p. 1240-9.

56. Lippi, G. and G. Cervellin, High-sensitivity troponin $T$ is more susceptible than highsensitivity troponin I to impaired renal function. Am J Cardiol, 2013. 112(12): p. 1985.

57. defilippi, C.R., J.A. de Lemos, R.H. Christenson, et al., Association of serial measures of cardiac troponin $\mathrm{T}$ using a sensitive assay with incident heart failure and cardiovascular mortality in older adults. JAMA, 2010. 304(22): p. 2494-502.

58. Haaf, P., T. Reichlin, R. Twerenbold, et al., Risk stratification in patients with acute chest pain using three high-sensitivity cardiac troponin assays. Eur Heart J, 2014. 35(6): p. 365-75.

59. de Antonio, M., J. Lupon, A. Galan, et al., Head-to-head comparison of high-sensitivity troponin $\mathrm{T}$ and sensitive-contemporary troponin I regarding heart failure risk stratification. Clin Chim Acta, 2013. 426: p. 18-24.

60. Mueller, M., S. Celik, M. Biener, et al., Diagnostic and prognostic performance of a novel high-sensitivity cardiac troponin $\mathrm{T}$ assay compared to a contemporary sensitive cardiac troponin I assay in patients with acute coronary syndrome. Clin Res Cardiol, 2012. 101(10): p. 837-45.

61. Bodor, G.S., D. Porterfield, E.M. Voss, et al., Cardiac troponin-I is not expressed in fetal and healthy or diseased adult human skeletal muscle tissue. Clin Chem, 1995. 41(12 Pt 1): p. 1710-5. 
62. Anderson, P.A., N.N. Malouf, A.E. Oakeley, et al., Troponin T isoform expression in humans. A comparison among normal and failing adult heart, fetal heart, and adult and fetal skeletal muscle. Circ Res, 1991. 69(5): p. 1226-33.

63. Apple, F.S., Tissue specificity of cardiac troponin I, cardiac troponin T and creatine kinaseMB. Clin Chim Acta, 1999. 284(2): p. 151-9.

64. Jaffe, A.S., V.C. Vasile, M. Milone, et al., Diseased skeletal muscle: a noncardiac source of increased circulating concentrations of cardiac troponin T. J Am Coll Cardiol, 2011. 58(17): p. 1819-24.

65. Rittoo, D., A. Jones, B. Lecky, et al., Elevation of cardiac troponin T, but not cardiac troponin $\mathrm{I}$, in patients with neuromuscular diseases: implications for the diagnosis of myocardial infarction. J Am Coll Cardiol, 2014. 63(22): p. 2411-20. 



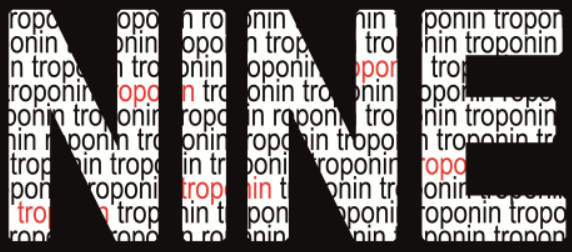

\section{SUMMARY}

\section{SAMENVATTING}





\section{Summary}

The diagnosis of acute myocardial infarction (AMI) is based on the release of cardiac troponins (cTn) in the bloodstream. Major analytical improvements to the clinical assays have led to more accurate and very sensitive information regarding cTn. However, the current high-sensitivity tests for cTnT (hs-cTnT) and cTnl (hs-cTnl) also resulted in the detection of elevated concentrations in patients with diseases other than $A M I$, such as heart failure, and renal dysfunction. This complicates the application of the assay and it is therefore of utmost importance to increase our understanding on how to interpret hs-cTn results, especially in the low-measuring range.

The diagnostic cTn cutoff for myocardial infarction is set at the 99th percentile upper reference limit (URL) of a healthy reference population. An overview of peer-reviewed literature on the real-life determinations of the 99th percentile cutoff is given in chapter two. This chapter illustrates that variable selection criteria are used to define the reference population, and subsequently a wide range of 99th percentile concentrations were found for each assay. Lack of attention regarding these issues can result in misleading decision cutoffs and can in this way influence clinical practice.

The association between kidney function and cTn concentrations has been described, although it is currently unclear whether chronic cTn elevations reflect chronic release due to cardiac damage or they are also the result of a decreased renal clearance. In chapter three, we therefore examined the extent that renal function contributes to higher cTn concentrations in patients with chest discomfort, in whom circulating troponins are mainly attributed to the presence and severity of atherosclerotic plaques or echocardiographic abnormalities. Remarkably, we revealed that the association with renal function was independent and stronger than with any measured CT parameters, such as CAD severity and coronary calcium score. Similarly, in an elderly population of nursing home residents (chapter four), with prevalent heart failure ( $40 \%)$ and renal dysfunction ( $54 \%$ ), hs-cTn concentrations, especially hs-cTnT, were highly associated with renal dysfunction even beyond the presence of heart failure. Together, these studies demonstrate that hs-cTn concentrations are highly and independently associated with both renal as cardiac diseases and 
subsequently both pathologies can greatly affect the 99th percentile URL. Consequently, the interpretation of baseline hs-cTn concentrations might be improved when accompanied with the assessment of renal clearance.

End-stage renal disease (ESRD) patients often exhibit elevated cTn levels that are more stable over time. To date, it remains unclear how dialysis affects cTn results, as both intradialytic increases and decreases have been reported. Chapter five is the first study to reveal the effects of extended hemodialysis and hemodiafiltration on cardiac markers and describes a significant reduction in hscTnT as well as hs-cTnl concentrations following hemodiafiltration.

Furthermore, there is evidence that cTn proteins are also subjected to posttranslational modifications, such as degradation. Which circulating CTnT forms are detected by the clinical assay in the blood of AMI and ESRD patients is investigated in chapter six and chapter seven, respectively. In chapter six, we show that, upon its release from the damaged myocardium, cTnT is degraded in a time-dependent manner, ranging from intact to low-molecular weight fragments. The smallest degradation products also accumulate in the circulation of ESRD patients (chapter seven). In this thesis, it was therefore clearly shown that the most prevalent molecular forms differed between AMI ( $29 \mathrm{kDa})$ and ESRD ( $<18 \mathrm{kDa})$ patients. It would therefore be interesting to investigate whether the clinical assay can be improved by detecting specific molecular cTnT forms.

Finally, throughout this thesis, hs-cTnT and hs-cTnl concentrations have been measured in various acute and non-acute settings, and we observed remarkable differences between hs-cTnT and hs-cTnl concentrations in all clinical populations, discussed in chapter eight. This seriously questions whether hs-cTnT and hs-cTnl results are truly interchangeable from each other. Future work should illustrate in more detail if a combination of both cTnT and cTnl testing can have added diagnostic value in more complex cases. 



\section{Samenvatting}

De diagnose van een acuut hartinfarct berust op de vrijzetting van cardiale troponines (cTn) in de bloedbaan. Door de ontwikkeling van meer accurate en gevoeligere cTn testen, zijn er steeds meer mensen waarbij cTn-verhogingen worden gedetecteerd. De huidige hoge gevoeligheidstesten voor cTnT (hscTnT) en cTnl (hs-cTnl) tonen aan dat beide testen ook verhoogd zijn bij patiënten met een andere pathologie als een myocardinfarct, zoals bijvoorbeeld bij hartfalen en renale dysfunctie. Dit alles bemoeilijkt het gebruik van de assay en roept vragen op over de interpretatie van cTnT en cTnl resultaten, vooral in het lage meetbereik.

De diagnostische afkapwaarde voor een myocardinfarct wordt gedefinieerd als de 99ste percentiel concentratie, gemeten in een gezonde referentiepopulatie. Een overzicht van de gepubliceerde literatuur over de werkelijke bepaling van deze 99ste percentiel afkapwaarde is weergegeven in hoofdstuk twee. Dit hoofdstuk illustreert dat in elke studie andere selectiecriteria gebruikt werden om de referentiepopulatie te definiëren. Dit heeft als gevolg dat steeds verschillende afkapwaarden gevonden werden voor éénzelfde assay. Gebrek aan aandacht voor deze verschillen kan resulteren in misleidende afkapwaarden en kan op deze manier de klinische diagnose beïnvloeden.

Dat er een associatie is tussen de nierfunctie en cTn-concentraties is reeds beschreven. Toch is momenteel niet geweten of deze chronische verhogingen het gevolg zijn van een ophoping van cTn moleculen door een gedaalde nierklaring of dat het om cardiale schade gaat. In hoofdstuk drie is daarom onderzocht in welke mate nierfunctie bijdraagt tot hogere cTn-concentraties bij patiënten met pijn op de borst. In deze populatie worden gedetecteerde cTnwaarden immers toegeschreven aan de aanwezigheid en ernst van coronaire atherosclerosis of echocardiografische afwijkingen. Opmerkelijk is dat in deze studie wordt aangetoond dat de associatie met de nierfunctie onafhankelijk en sterker was dan de associatie met CT-parameters, zoals de ernst van de atherosclerose en een hoge coronaire calcificatie score. In een populatie van fragiele verpleeghuisbewoners (hoofdstuk vier), met een hoge prevalentie van hartfalen ( $\sim 40 \%$ ) en nierfunctiestoornissen ( $\sim 4 \%)$, wordt dit ook duidelijk. Hier tonen we aan dat hs-cTn concentraties, met name hs-cTnT, zeer sterk 
geassocieerd zijn met een gedaalde nierfunctie, onafhankelijk van de aanwezigheid van hartfalen. Samen laten deze studies zien dat hs-cTn concentraties niet enkel hart- maar ook nieraandoeningen kunnen weerspiegelen en dat vervolgens beide pathologieën een grote invloed hebben op de $99^{\text {ste }}$ percentiel afkapwaarde. Bijgevolg zou de interpretatie van hs-cTn concentraties kunnen worden verbeterd wanneer zij gecombineerd worden met een schatting van de renale klaring.

Patiënten aan dialyse vertonen vaak verhoogde cTn resultaten die meer stabiel zijn over de tijd. Tot op heden blijft onduidelijk hoe dialyse de cTn resultaten kan beïnvloeden, gezien zowel cTn stijgingen als dalingen gemeten worden na dialyse. Hoofdstuk vijf is de eerste studie die de effecten van langere hemodialyse- en hemodiafiltratie-behandeling onderzoekt. Deze studie laat zien dat een significante daling in hs-cTnT en hs-cTnl concentraties aanwezig is na hemodiafiltratie.

Tevens is er bewijs dat cTn eiwitten ook post-translationele modificaties, zoals degradatie, kunnen ondergaan. De verschillende cTnT vormen, in het bloed van hartinfarct en dialyse patiënten, die gedetecteerd kunnen worden door de huidige klinische assay worden onderzocht in respectievelijk hoofdstuk zes en hoofdstuk zeven. In hoofdstuk zes tonen we aan dat na een myocardinfarct, cTnT gedegradeerd voorkomt in het bloed. Deze degradatie gebeurt in een tijdsafhankelijke patroon, waarbij eerst intact en vervolgens laag moleculaire cTnT-fragmenten gedetecteerd worden. In hoofdstuk zeven wordt echter bewezen dat in de bloedcirculatie van dialyse-patiënten enkel deze kleine afbraakproducten aanwezig zijn. In dit proefschrift wordt daarom duidelijk dat de gedetecteerde moleculaire cTnT-vormen gedurende de eerste uren na een hartinfarct ( $29 \mathrm{kDa}$ ) verschillen van dialyse-patiënten (<18 kDa). Het zou daarom zeer interessant zijn om te onderzoeken of de huidige cTnT-test verbeterd kan worden door specifiekere cTnT-vormen te detecteren, zoals het 29-kDa fragment.

Tot slot werd in dit proefschrift hs-cTnT en hs-cTnl gemeten in diverse populaties met acute en niet-acute pathologieën. Hierbij zagen we opmerkelijke verschillen tussen hs-cTnT en hs-cTnl, zoals bediscussieerd in hoofdstuk acht. Deze bevindingen stellen daarom in vraag of de resultaten van de cTnT en cTnl 
assays daadwerkelijk uitwisselbaar zijn van elkaar. Verdere studies moeten in meer detail uitwijzen of een combinatie van beide testen een diagnostische meerwaarde kunnen betekenen in patiënten met meer complexere ziektebeelden. 




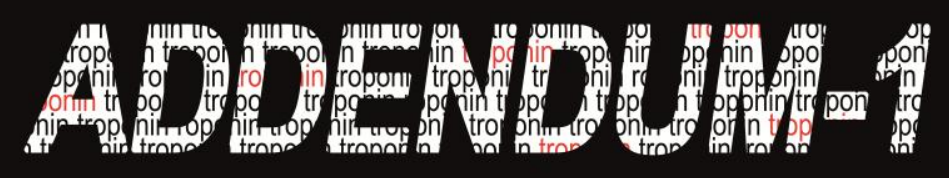

\section{VALORISATION}

\section{ABBREVIATIONS}





\section{Valorisation}

\section{Economic and Social Relevance}

Cardiovascular diseases (CVD) are a major cause of death with a mortality rate of approximately $30 \%$ across Europe and America [1, 2]. CVD covers a wide range of disorders, including diseases of the cardiac muscle and of the vascular system. The majority of CVD that lead to mortality are due to acute myocardial infarction (AMI) and stroke. Cardiovascular research has already resulted into more interventions with significant reductions in morbidity and mortality [3, 4]. Regardless of this decline in mortality, more patients are at cardiovascular risk due to increased life expectancy and comorbidities related to an unhealthy lifestyle. Clinical care of these patients is already costly and is expected to further increase in the future. Additionally, treatments may also tremendously impact the quality of life of these patients. Future research should therefore focus on prevention and earlier detection of heart diseases.

Cardiac troponins are an essential component of the diagnostic criteria for myocardial infarction, especially when electrocardiographic results are inconclusive. According to the current guidelines a rise and/or fall in cardiac troponin (cTn) T or I concentrations, with at least one value above the $99^{\text {th }}$ percentile of a healthy reference population, signifies myocardial damage and must be accompanied with also clinical indications such as ischemic symptoms or imaging evidence of myocardial damage [5].

Increasingly sensitive cTn assays enable an earlier diagnosis but severely impair the specificity towards AMI. When measuring with high-sensitivity (hs-) assays, elevated hs-cTn concentrations were also detected in a whole range of other pathologies [6]. Due to this lack in disease-specificity, unravelling the causes of hs-cTn positive results can therefore be challenging, especially in patients affected by more than one disease.

On the contrary, the increases in analytical sensitivity also gave rise to new opportunities for these assays. Even below the diagnostic cutoff, higher hs-cTn values are strongly associated with an increased risk on cardiovascular diseases and mortality, making them promising risk stratifying tools $[7,8]$. 


\section{Target groups}

Cardiac troponins are important and highly used cardiac markers and their results are mainly interpreted by clinicians and laboratory specialists. Even before the publication of the current guidelines [5], a European survey demonstrated that $59 \%$ of the inquired laboratories were using cTnT or cTnl concentrations to diagnose AMI [9]. Results presented in this thesis can guide clinicians and laboratory specialists to a better understanding of hs-cTn results, by gaining insight into important clinical questions such as changes in renal clearance can affect hs-cTn results. Eventually a better interpretation of hs-cTn results can lead to an earlier diagnosis or rule out of patients. These advancements all have the potential to lead to more efficient, better and cost effective diagnostic process.

\section{Activities and/or products}

The results described in this thesis can be used for the development of various products or tools.

In this thesis we show that elevated cTn, especially hs-cTnT, concentrations were detected in elderly people without any evidence of heart and kidney diseases. These results support the concept that also other clinical indicators, besides acute myocardial damage, can greatly influence baseline hs-cTn results. As such, when considering individuals with non-acute symptoms, a young female individual without any cardiac history and a normal renal clearance will have lower baseline cTn concentrations as compared to an older male individual with decreases in renal clearance. For this reason, applying generalized cutoffs can result in under- or over-diagnosis within different patient cohorts [10-12], already implying that it is of great importance to interpret hs-cTn results to a more individual level. The development of a clinical alghorithm might therefore be helpful to give direction of which hs-cTn result to expect in a patient, taken into account important factors (such as age, gender, 
cardiovascular history, renal clearance, timing of blood collection, precision of the assay, etc.) Current guidelines also indicate the advantage of measuring more than one cTn concentration in time, although it remains unclear which delta to use for a safe rule out of a patient [5, 13-15]. Also here, a clinical algorithm might be helpful for a better indication what constitutes a significantly increased value that requires further clinical examinations.

Although cTn results are currently not used in the clinic to indicate the risk on future cardiovascular diseases and mortality, all current literature undeniably shows that higher troponin concentrations are significantly associated with the incidence of such a risk [16]. It seems therefore quite logic that hs-cTn results should be implemented in clinical risk scores that can be used in patients at risk for cardiovascular diseases. Potentially these clinical risk scores can serve guidance for preventive therapies in patients at high risk.

Furthermore, measuring multiple biomarkers have been proposed in the management of acute coronary syndromes. The combination of cTn measurements with more traditional biomarkers (C-reactive proteins, NTproBNP) and newly developed biomarkers (such as copeptin, heart-type fatty acid-binding protein) are widely investigated [17-19]. In this thesis, preliminary data shows that combining hs-cTnT and hs-cTnl assays might also be promising to further enhance the diagnostic potential of cardiac troponins.

We are the first to clearly show that the most prevalent molecular cTnT forms detected by the clinical cTnT assay are fragments. Moreover, different cTnT forms were observed during the early acute cTnT release following AMI ( 29 $\mathrm{kDa})$ as compared to chronic elevations seen in ESRD $(<18 \mathrm{kDa})$ patients. New and unpublished results show that larger cTnT forms ( $\geq 29 \mathrm{kDa}$ ) still contain their C-terminal end, while the smaller fragments $(<18 \mathrm{kDa})$ do not. This thesis therefore indicates that targeting the larger $\mathrm{CTnT}$ fragments have the potential to enhance the disease-specificity of the clinical assay. Adding a third antibody, directed against the C-terminal region of CTnT, to the current clinical ELISA 
method can detect the proportion of these larger cTnT fragments, as illustrated in FIGURE 1. Future research has to indicate whether such a test can be used in addition to the current clinical hs-cTnT test, and might lead to a better differentiation between acute and chronic cTnT elevations.

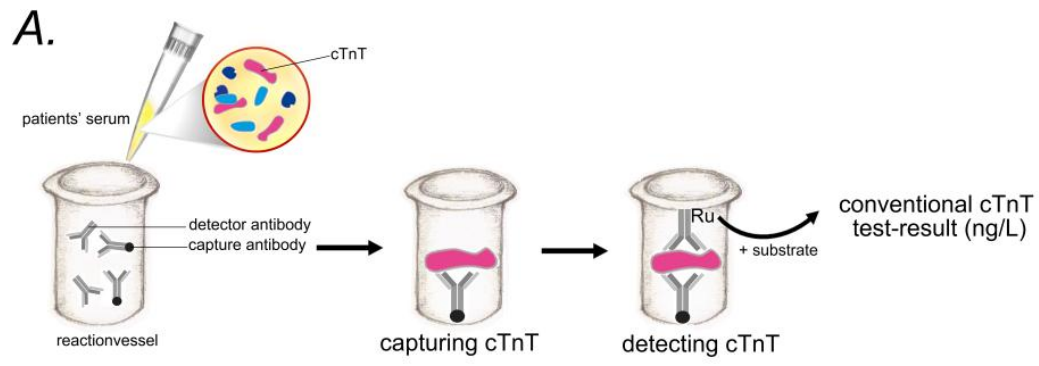

$B$.

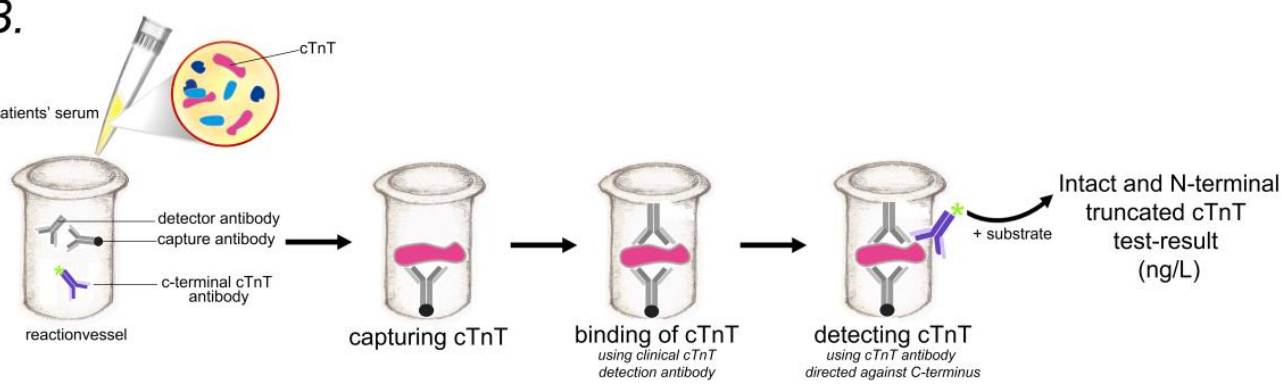

FIGURE 1. A. Conventional CTnT testing according to the ELISA method of the clinical assay. B. Incorporation of a third cTnT antibody directed against the $\mathrm{C}$-terminal end.

\section{Implementation in clinical practice}

Cardiac troponins are already incorporated in clinical practice. Results obtained in this thesis show promising new opportunities for this biomarker in the clinic. Over the past years, increasingly more research is performed on the determinants and risk-stratification of hs-cTn results. However, specific algorithms incorporating more clinical indicators for high hs-cTn results are currently not available and still need to be developed. Also, many clinical scores have already been developed for the prediction of adverse events in high-risk 
patiens with ACS [20, 21], low-risk patients with symptoms of chest pain [22, 23] and even more general populations [24, 25]. However, most of these risk scores do not include hs-cTn results. Up till now, only one risk algorithm incorporates cTn concentrations when determining the risk in patients presenting with chest pain at the emergency department [23], which is still validated in the clinic trials. Whether hs-cTn concentrations can further improve these risk scores, also in more general populations, still merits further research.

Furthermore, the development of a new assay entails careful validation of the quantification, linearity and specificity of the results. To ensure the detection of cardiospecific cTnT isoforms, we propose the inclusion of a third antibody to the current hs-cTnT assay. For this reason, this new method must be developed in close collaboration with the manufacturer of the current hs-cTnT test (Roche Diagnostics).

Once these advancements are developed, the added value should be monitored in prospectively randomized control studies. Eventually, correct implementation of these tools requires that specialists should be educated on how to interpret the results through scientific exposure (peer-reviewed publications or international congresses) and guideline documents that are published by national and international societies in cardiology and biochemistry. 


\section{References}

1. Nichols, M., N. Townsend, P. Scarborough, et al. European Cardiovascular Disease Statistics. European Society of Cardiology (www.escardio.org).

2. Go, A.S., D. Mozaffarian, V.L. Roger, et al., Heart disease and stroke statistics--2014 update: a report from the American Heart Association. Circulation, 2014. 129(3): p. e28-e292.

3. Heart Protection Study Collaborative, G., MRC/BHF Heart Protection Study of cholesterol lowering with simvastatin in 20,536 high-risk individuals: a randomised placebo-controlled trial. Lancet, 2002. 360(9326): p. 7-22.

4. Keeley, E.C., J.A. Boura, and C.L. Grines, Primary angioplasty versus intravenous thrombolytic therapy for acute myocardial infarction: a quantitative review of 23 randomised trials. Lancet, 2003. 361(9351): p. 13-20.

5. Thygesen, K., J.S. Alpert, A.S. Jaffe, et al., Third universal definition of myocardial infarction. Eur Heart J, 2012. 33(20): p. 2551-67.

6. Kelley, W.E., J.L. Januzzi, and R.H. Christenson, Increases of cardiac troponin in conditions other than acute coronary syndrome and heart failure. Clin Chem, 2009. 55(12): p. 2098112.

7. de Lemos, J.A., M.H. Drazner, T. Omland, et al., Association of troponin T detected with a highly sensitive assay and cardiac structure and mortality risk in the general population. JAMA, 2010. 304(22): p. 2503-12.

8. Mills, N.L., A.M. Churchhouse, K.K. Lee, et al., Implementation of a sensitive troponin I assay and risk of recurrent myocardial infarction and death in patients with suspected acute coronary syndrome. JAMA, 2011. 305(12): p. 1210-6.

9. Collinson, P.O., M.P. van Dieijen-Visser, K. Pulkki, et al., Evidence-based laboratory medicine: how well do laboratories follow recommendations and guidelines? The Cardiac Marker Guideline Uptake in Europe (CARMAGUE) study. Clin Chem, 2012. 58(1): p. 305-6.

10. Shah, A.S., M. Griffiths, K.K. Lee, et al., High sensitivity cardiac troponin and the underdiagnosis of myocardial infarction in women: prospective cohort study. BMJ, 2015. 350: p. g7873.

11. Reiter, M., R. Twerenbold, T. Reichlin, et al., Early diagnosis of acute myocardial infarction in the elderly using more sensitive cardiac troponin assays. Eur Heart J, 2011. 32(11): p. 137989.

12. Olivieri, F., R. Galeazzi, D. Giavarina, et al., Aged-related increase of high sensitive Troponin $\mathrm{T}$ and its implication in acute myocardial infarction diagnosis of elderly patients. Mech Ageing Dev, 2012. 133(5): p. 300-5.

13. Reichlin, T., A. Irfan, R. Twerenbold, et al., Utility of absolute and relative changes in cardiac troponin concentrations in the early diagnosis of acute myocardial infarction. Circulation, 2011. 124(2): p. 136-45.

14. Mueller, M., M. Biener, M. Vafaie, et al., Absolute and relative kinetic changes of highsensitivity cardiac troponin $\mathrm{T}$ in acute coronary syndrome and in patients with increased troponin in the absence of acute coronary syndrome. Clin Chem, 2012. 58(1): p. 209-18.

15. Keller, T., T. Zeller, F. Ojeda, et al., Serial changes in highly sensitive troponin I assay and early diagnosis of myocardial infarction. JAMA, 2011. 306(24): p. 2684-93.

16. Newby, L.K., R.L. Jesse, J.D. Babb, et al., ACCF 2012 expert consensus document on practical clinical considerations in the interpretation of troponin elevations: a report of the American College of Cardiology Foundation task force on Clinical Expert Consensus Documents. J Am Coll Cardiol, 2012. 60(23): p. 2427-63.

17. Sabatine, M.S., D.A. Morrow, J.A. de Lemos, et al., Multimarker approach to risk stratification in non-ST elevation acute coronary syndromes: simultaneous assessment of troponin I, C-reactive protein, and B-type natriuretic peptide. Circulation, 2002. 105(15): p. 1760-3.

18. Kellens, S., F.H. Verbrugge, M. Vanmechelen, et al., Point-of-care heart-type fatty acid binding protein versus high-sensitivity troponin $T$ testing in emergency patients at high risk for acute coronary syndrome. Eur Heart J Acute Cardiovasc Care, 2015. 
19. Jacobs, L.H., M. van Borren, E. Gemen, et al., Rapidly rule out acute myocardial infarction by combining copeptin and heart-type fatty acid-binding protein with cardiac troponin. Ann Clin Biochem, 2015.

20. Granger, C.B., R.J. Goldberg, O. Dabbous, et al., Predictors of hospital mortality in the global registry of acute coronary events. Arch Intern Med, 2003. 163(19): p. 2345-53.

21. Boersma, E., K.S. Pieper, E.W. Steyerberg, et al., Predictors of outcome in patients with acute coronary syndromes without persistent ST-segment elevation. Results from an international trial of 9461 patients. The PURSUIT Investigators. Circulation, 2000. 101(22): p. 2557-67.

22. Antman, E.M., M. Cohen, P.J. Bernink, et al., The TIMI risk score for unstable angina/non-ST elevation MI: A method for prognostication and therapeutic decision making. JAMA, 2000. 284(7): p. 835-42.

23. Backus, B.E., A.J. Six, J.H. Kelder, et al., Risk scores for patients with chest pain: evaluation in the emergency department. Curr Cardiol Rev, 2011. 7(1): p. 2-8.

24. D'Agostino, R.B., Sr., R.S. Vasan, M.J. Pencina, et al., General cardiovascular risk profile for use in primary care: the Framingham Heart Study. Circulation, 2008. 117(6): p. 743-53.

25. Assmann, G., P. Cullen, and H. Schulte, Simple scoring scheme for calculating the risk of acute coronary events based on the 10-year follow-up of the prospective cardiovascular Munster (PROCAM) study. Circulation, 2002. 105(3): p. 310-5. 



\section{Abbreviations}

AMI

CAD

cTn

cTnT

cTnl

$\mathrm{TnC}$

hs-cTn

hs-cTnT

hs-cTnl

ECG

STEMI

NSTEMI

URL

CV

CKD

ESRD

ACS

LoB

LoD

$\mathrm{RCV}$

CVD

CCTA

CCS

eGFR

LVH

LVEF

IQR

ROC

AUC

$\mathrm{Cl}$

CABG acute myocardial infarction

coronary artery disease

cardiac troponin

cardiac troponin $\mathrm{T}$

cardiac troponin I

troponin C

high-sensitivity cardiac troponin

high-sensitivity cardiac troponin $\mathrm{T}$

high-sensitivity cardiac troponin I

electrocardiography

ST-elevation myocardial infarction

non-ST elevation myocardial infarction

upper reference limit

coefficient of variation

chronic kidney disease

end-stage renal disease

acute coronary syndromes

limit of blank

limit of detection

reference change value

cardiovascular disease

cardiac computed tomographic angiography

coronary calcium score

estimated glomerular filtration rate

left ventricular hypertrophy

left ventricular ejection fraction

interquartile range

receiver-operating curve

area-under-the-curve

confidence interval

coronary artery bypass graft 


$\begin{array}{ll}\text { PCI } & \begin{array}{l}\text { percutaneous coronary intervention } \\ \text { NTproBNP }\end{array} \\ \text { OR } & \begin{array}{l}\text { natriuretic peptide } \\ \text { odds ratio } \\ \text { hR }\end{array} \\ \text { HD } & \text { hemord ratio } \\ \text { HDF } & \text { hemodiafiltration } \\ \text { RR } & \text { reduction ratio } \\ \text { GFC } & \text { gel filtration chromatography } \\ \text { CK } & \text { creatine kinase } \\ \text { LD } & \text { lactate dehydrogenase } \\ \text { MW } & \text { molecular weight } \\ \text { V0 } & \text { dead volume } \\ \text { VR } & \text { retention volume } \\ \text { Kav } & \text { gel-phase distribution coefficient } \\ \text { ED } & \text { emergency department } \\ \text { PBS } & \text { phosphate buffered saline }\end{array}$






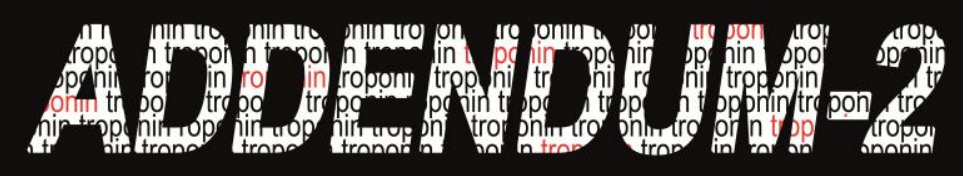

\section{DANKWOORD/ACKNOWLEDGMENTS}

\section{CURRICULUM VITAE}



Tenslotte wil ik van dit hoofdstuk graag gebruik maken om iedereen te bedanken voor zijn/haar bijdrage aan deze thesis, waarvan ik enkele mensen speciaal wil benoemen.

Op de eerste plaats wil ik graag mijn promotor Prof. dr. van Dieijen-Visser en co-promotoren Prof. dr. Bekers en Dr. Mingels bedanken voor de fijne samenwerking, het vertrouwen dat jullie steeds in mij hadden en de tijd die jullie elke keer vrij maakte voor onze meetings. Ik heb ontzettend veel opgestoken tijdens deze leerzame periode.

Beste Marja, het was een hele eer om met je samen te werken. Het voelt daarom voor mij nog steeds heel onnatuurlijk aan om iemand waar ik zo naar opkijk bij de voornaam en met de je-vorm aan te spreken.

Beste Otto, jouw deur stond altijd open voor mij als ik je hulp nodig had. Met een dosis humor, een koekje of pepermuntje wist je het altijd gezellig te maken tijdens ons overleg.

Beste Alma, toen ik pas begon, zat jij reeds in de eindfase van je promotie. Al heel snel vond ik mijn weg richting de vervolgstudies van jouw projecten. Dit had als resultaat dat ook een groot deel van mijn begeleiding op jou terechtkwam. Hoe je het allemaal naast je opleiding wist te combineren blijft me nog steeds een raadsel. Je hebt me altijd mijn eigen ding laten doen maar mij toch ook bijgestaan waar ik het nodig had. Ik hoop dat je nog een succesvolle toekomst tegemoet gaat als Klinisch Chemicus, samen met je mooi gezinnetje.

Graag wil ik van de gelegenheid gebruik maken om alle leden van mijn beoordelingscommissie hartelijk te bedanken voor het snelle en positieve oordeel en Prof. dr. van Loon voor zijn bereidwilligheid om te fungeren als voorzitter van mijn beoordelingscommissie. Prof. dr. Mueller, unfortunately you are not able to come to my PhD defense, but I would like to sincerely thank you for taking the time to be a member of my assessment comittee.

Het onderzoek in dit proefschrift is het resultaat van enkele zeer vruchtbare samenwerkingen met mensen van diverse klinische afdelingen binnen dit ziekenhuis. Zo wil ik graag van de afdeling Cardiologie Dr. Kietselaer, Sibel 
Altintas, Ivo Joosen en Mathijs Versteylen bedanken voor de fijne samenwerking. Als ik iets nodig had van jullie werd dit altijd zeer snel geregeld en ik heb veel van jullie klinische kennis opgestoken. Ik hoop dat onze gezamenlijke papers zeer vlug geaccepteerd worden. Prof. dr. Kooman, Tom Cornelis en Natascha Broers van de afdeling Nefrologie, bedankt voor de zeer fijne en productieve samenwerking. Ook wil ik Marielle van der Velden, Prof dr. Schols en Prof dr. Brunner-La Rocca bedanken voor de aangename en leerrijke samenwerking tijdens de verpleeghuizen-studie.

Ook buiten Maastricht, wil ik graag Prof. dr. van Suijlen, Marijke Niens en Laurens-Jan Jellema bedanken voor de hulp en het ter beschikking stellen van jullie lab tijdens de velen hs-cTnl bepalingen, en Dr. ten Kate voor de mogelijkheid om ook in Sittard troponine I metingen uit te voeren.

Ook dank aan alle andere co-auteurs die ik niet bij naam genoemd heb maar wel hebben bijgedragen aan dit onderzoek.

Dan kom ik bij mijn collega-promovendi en overige leden van het troponineclubje. Lieve Lieke, jij vervoegde ons team direct na mij en we hadden het al heel snel zeer gezellig met ons tweetjes. Bedankt voor de goede raad en de inzichtvolle discussies. We hebben zeer fijne tijden beleefd, waaronder de leuke congressen. Ik ben heel vereerd dat je op de grote dag als paranimf naast mij staat! Momenteel zit je ook zelf in de eindfase van je promotie, en wens ik je nog veel succes met de laatste loodjes! Noreen, dankzij jou bleef ik altijd op de hoogte van alle nieuwtjes van Maastricht in omstreken. Sander, het was voor jou niet altijd eenvoudig om de haan in ons kippenhok te zijn. Dorien, Judith en Frederique, de laatste nieuwkomers in het clubje, maar zeker niet de minste! Ik wens jullie allemaal heel veel succes toe met jullie onderzoek! Vincent, het manusje van alles op het lab. Je had altijd wel een creatieve oplossing voor de problemen tijdens het meten. Ik wil je heel erg bedanken voor alle hulp die je mij gegeven hebt bij alle bepalingen, niet enkel in Maastricht maar ook in Apeldoorn en Sittard. Steven, bedankt voor alle hulp en input. Ik heb ontzettend veel van je geleerd.

Ook alle overige stafleden van het CDL wil ik graag bedanken voor alle waardevolle inzichten tijdens presentaties, journal clubs, etc... 
Petal, zoals jij bestaan er geen twee. Je hebt me veel orde en netheid bijgebracht in het lab $\odot$. Bedankt voor de leuke tijd. Richard, een welgemeende dank $u$ voor het in goede banen leiden van mijn vele en soms ook 'exotische` bestellingen. Maurice, als ik weer iets nodig had dan was dit nooit te veel moeite voor je. Bedankt hiervoor en voor alle interesse die je altijd toonde. Annita, dikke merci voor de leuke gesprekken en voor alle hulp op het SIRC. Marianne, Joyce, Henrike en de dames van kwaliteit, het was altijd gezellig met jullie tijdens de lunch.

Ook wil ik Anneke van Sleeuwen bedanken voor haar enorme inzet tijdens haar stage en vakantiewerk bij ons op het lab. Beste Anneke, zonder jouw hulp was hoofdstuk 7 nooit helemaal afgeraakt. Ik wens je het allerbeste toe voor de toekomst.

Sylvia Masotti, you came to our lab in the most busiest period of my PhD. It was very nice to meet you and to see your interest in our chromatography-project. I wish you al the luck with your own research!

Tot slot nog een woordje van dank voor mijn voorgangers en ex-collega`s Leo Jacobs, Bianca Pulinx en Lenneke Prinzen, voor het goede voorbeeld dat ze altijd gaven en iedereen op het Centraal Diagnostisch Laboratorium die ik nog verder vergeten ben en mij bij het een en het ander hebben geholpen.

Natuurlijk zijn er ook buiten het werk heel wat mensen van wie ik steun gekregen heb tijdens deze intensieve jaren.

Lieve Evi, Karen en Lize, jullie weten als geen ander wat er voor nodig is om tot dit resultaat te komen. Heel erg bedankt dat jullie er altijd voor me waren en me met raad en daad hebben bijgestaan. Evi, het was altijd heel erg gezellig bij jou in Londen! Ik bewonder alles wat je doet. Eender waar je besluit te wonen (al hoop ik ooit nog eens terug België (-)), we blijven vriendinnen en ik kom je met heel veel plezier bezoeken. Karen, lieve meid, we denken op veel vlakken hetzelfde en bedankt voor alle leuke gesprekken die daardoor ontstaan. Ik ben supertrots op alles wat je al bereikt hebt in je leven. Lize, ik heb altijd veel bewondering gehad hoe je alles moeiteloos uitvoert. Tijdens mijn verdediging, ben jij in betere oorden (een tikkeltje jaloezie van deze kant (-)) en ik hoop dat je een geweldige reis tegemoet gaat. 
Ook een welgemeende dank jullie wel voor mijn allerliefste vriendinnen Lore, Tine, Anouk, Lindsay en Sofie voor alle leuke, gezellige en opbeurende brunchkes, avonden, etc.. Ik ben er zeker van dat we nog heel lang vriendinnen blijven! Ik apprecieer het echt enorm dat jullie de moeite nemen om er 10 juli bij te zijn, dan kunnen jullie ook eindelijk eens zien waar ik al die tijd aan gewerkt heb.

Naast mijn familie wil ik ook mijn schoonfamilie heel erg bedanken voor alle steun en interesse!

Dorine, lieve zus, het was niet altijd fijn voor je om op te groeien met een kleine zus die achter je na huppelde en alles wilde wat jij had en deed. Maar toen ik (net als jij) op universiteit een wetenschappelijke richting koos, was je er altijd voor mij en heb je mij steeds ten volle gesteund. Ik ben ook ontzettend vereerd dat ik jouw getuige ben bij je trouw en dat je de wederdienst doet als paranimf op mijn verdediging.

Mama en papa, ik heb het jullie niet altijd gemakkelijk gemaakt met mijn keuzes en eigenwijsheid, maar jullie bleven me altijd onvoorwaardelijk steunen. Hoe ouder ik word, hoe meer ik dit weet te appreciëren. Ik kan me geen betere ouders voorstellen.

Mijn lief moeke-tje, al ben je er niet meer, ik weet dat je 10 juli naast me staat. Lieve Bert, bij het schrijven van jouw stukje besef ik eigenlijk hoe slecht ik in deze dingen ben. Een simpele dank $u$ wel volstaat immers niet om je te bedanken voor die ontelbare keren dat je er voor mij was, voor al de steun die je mij gaf, de rust die je uitstraalde in de stressvolle periodes, de hulp die je mij gaf wanneer ik weer eens duizenden buisjes moest stickeren en honderden epjes moest labelen, het nalezen van mijn Noyons beursaanvraag, en ga zo maar door... Ik hoop daarom dat je dit boekje ook als jouw verdienste ziet, met 10 juli de kroon op al het werk, en dat we samen nog veel gelukkige momenten mogen beleven. 




\section{CURRICULUM VITAE}

$\begin{array}{ll}\text { Name } & \text { Eline Cardinaels } \\ \text { Date of Birth } & \text { June 18th 1986 } \\ \text { Place of Birth } & \text { Bree, Belgium } \\ \text { Nationality } & \text { Belgian }\end{array}$

\section{PROFESSIONAL EXPERIENCE}

\section{0-2015 PhD at the department of Clinical Chemistry}

Central Diagnostic Laboratory - Cardiovascular Research Institute Maastricht (CARIM) - Maastricht University Medical Center+ (MUMC+)

\section{8-2009 Master Internships}

- Department of Pathology, Maastricht University Medical Center+ (MUMC+)

Project: Determination of mechanisms leading to trastuzumab resistance in breast tumor patients

- Department of Neurosurgery and Radiology - Ziekenhuis Oost-Limburg (Belgium)

Project: Peroneal Neuropathy after weight loss: histological and ultrasonographic characterization of the $n$. fibularis communis

\section{EDUCATION}

\section{9-2010 Pharmaceutical Sciences \\ Katholieke Universiteit Leuven (KULeuven), Belgium \\ 2008-2009 Master in Clinical and Molecular Sciences \\ Hasselt University (UHasselt), Belgium (graduated with \\ distinction)}

2004-2008 Bachelor in Biomedical Sciences

Hasselt University (UHasselt), Belgium

1998-2004 Sciences and Mathematics (8 h/week)

Onze-Lieve-Vrouw Lyceum Genk, Belgium 


\section{GRANTS}

- Winner of the Noyons research grant $2014(€ 15.000)$ - Spring congres of the Dutch association of Clinical Chemistry (NVKC) - Veldhoven

- Congress Bursary winner 2013 (€300) - Euromedlab congress of the International Federation of Clinical Chemistry (IFCC) , Milan, Italy

\section{PUBLICATION LIST}

- Cardinaels E, Mingels A, van Rooij T, Collinson P, Prinzen F, van DieijenVisser $M$. Time-dependent degradation pattern of cardiac troponin $T$ following myocardial infarction. Clinical Chemistry 2013; 59: 1083-1090. (impact factor 7.8)

- Cardinaels E, Mingels A,Jacobs L, Meex S, Bekers O and van DieijenVisser M. A comprehensive review of upper reference limits reported for (high-)sensitivity cardiac troponin assays: the challenges that lie ahead. Clin Chem Lab Med 2012, 50: 791-806. (impact factor 3)

- Cardinaels E, Cornelis T, van der Sande F, Eloot S, Damoiseaux J, Leunissen K, Kooman J, Bekers O. Acute effects of conventional and extended hemodialysis and hemodiafiltration on cardiac biomarkers. CCLM 2015 [in press] (impact factor 3)

- Cardinaels E, Altintas S, Versteylen M, Joosen I, Jellema L, Das M, Wildberger J, Crijns $H$, Bekers $O$, van Dieijen-Visser $M$, Kietselaer $B$, Mingels A. High-sensitivity cardiac troponin concentrations in patients with chest discomfort: is it the heart or the kidneys? [submitted to PLOS ONE]

- Cardinaels E, van der Velden M, Bekers O, ten Kate J, van Suijlen J, Niens $M$, van Dieijen-Visser M, Brunner-La Rocca HP, Schols J, Mingels A Cardiac troponin $\mathrm{T}$, but not troponin I, is elevated in aged nursing home residents and what are the clinical implications? [Submitted to J. Am Dir. Assoc.]

- Meylaerts L, Cardinaels E, Vandevenne J, Velghe B, Gelin G, Vanormelingen L, Weyns F. Peroneal neuropathy after weight loss: A highresolution ultrasonographic characterization of the common peroneal nerve. Skeletal Radiology 2011; 40: 1557-62. (impact factor 1.7)

- Cornelis T, van der Sande F, Eloot S, Cardinaels E, Bekers O, Damoiseaux J, Leunissen K, Kooman J. Acute Hemodynamic Response and Uremic Toxin Removal in Conventional and Extended Hemodialysis and Hemodiafiltration: A Randomized Crossover Study. Am J Kidney Dis. 2014 (impact factor 5.6) 
- Streng A, Jacobs L, Schwenk R, Cardinaels E, Meex S, Wodzig W, van Dieijen-Visser M Cardiac troponin in ischemic cardiomyocytes: Intracellular decrease before onset of cell death. Exp Mol Pathol. 2014; 96: 339-345 (impact factor 2.8)

- van Dieijen-Visser M, Mingels A, Jacobs L, Cardinaels E, Wodzig W, Bekers O, Klinkenberg L, Meex S. Troponin, the best there is! Nederlands tijdschrift voor klinische chemie en laboratoriumgeneeskunde 2012; 37: 1518 


